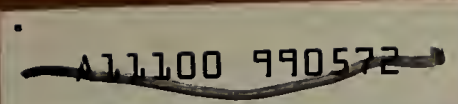

NASIR 80-2143

\title{
An Analysis of the Effects of Dynamic and Static Forces Present in the NBS SI Volt Experiment
}

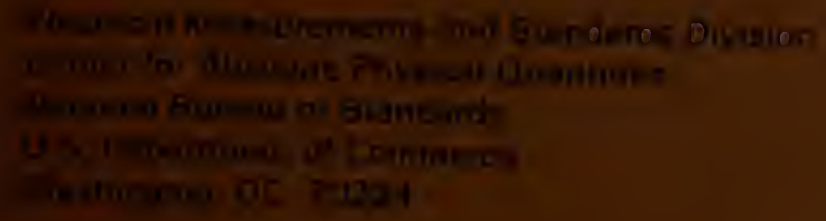


. 


\section{AN ANALYSIS OF THE EFFECTS DYNAMIC AND STATIC FORCES \\ PRESENT IN THE NBS SI VOLT EXPERIMENT

M. E. Cage

Electrical Measurements and Standards Division Center for Absolute Physical Quantities

National Bureau of Standards

U.S. Department of Commerce

Washington, DC 20234

Issued September 1980

Final Report

U.S. DEPARTMENT OF COMMERCE, Philip M. Klutznick, Secretary Luther H. Hodges, Jr., Deputy Secretary Jordan J. Baruch, Assistant Secretary for Productivity, Technology, and Innovation NATIONAL BUREAU OF STANDARDS, Ernest Ambler, Director 


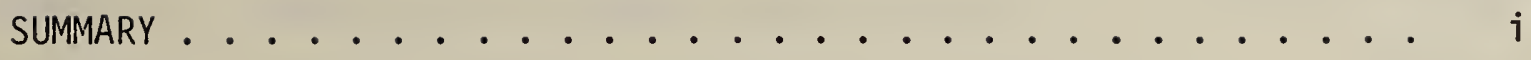

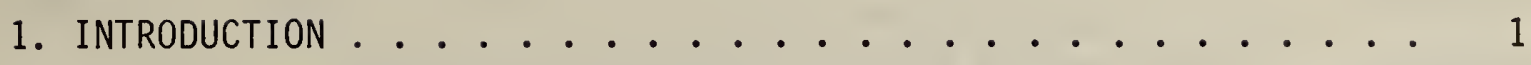

1.1 Objectives and order of the analysis......... 1

1.2 Description of the SI volt experiment ........ 3

2. CAPACITANCE AND ELECTRICAL FORCE EQUATIONS ......... 7

2.1 Coordinate system for the balance ........ 7

2.2 Capacitance equations for individual eléctrode

displacements or tilts ............. 9

2. 3 Coordinate system for the relative electrode positions . . 10

2.4 The capacitance equation ............. 12

2.5 The force equations ............. . . . 13

3. THE EQUATIONS OF MOTION AND THEIR SOLUTIONS ........ 16

3.1 Generalized torques .............. 16

3.2 The system in static equilibrium .......... 18

3.3 Equations of motion and their solutions ....... 19

3.4 Verification of the equations of motion ....... 23

3.5 Dynamical capacitance measurements .......... . 24

4. THE SLOPE AND PATH INTEGRAL METHODS WITH POSSIBLE ERRORS . . . 28

4.1 The slope method .................. 28

4.2 The path integral method ............. 33

5. RESULTS AND CONCLUSIONS ......................... 38

5.1 Problems in measuring $\hat{\mathrm{F}}_{\mathrm{z}} \ldots \ldots . \ldots 38$

5.2 Problems in measuring $\hat{C} \ldots \ldots 45$

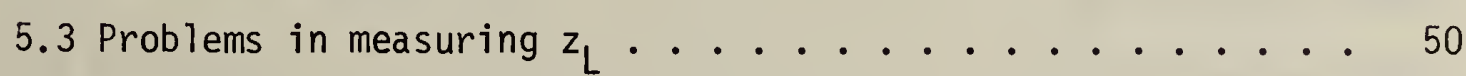

5.4. Problems in the slope and path' integral methods ..... 52 
ACKNOWLEDGEMENTS ................................ Page

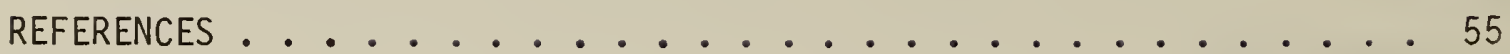

APPENDIX A. CAPACITANCE OF RADIALLY DISPLACED CYLINDRICAL

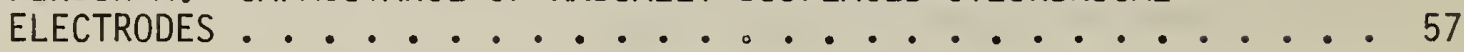

APPENDIX B. CAPACITANCE OF TILTED CYLINDRICAL ELECTRODES . . . 61

APPENDIX C. VARIATIONS AND UNCERTAINTIES OF $z_{L}$ AND $C \ldots 66$

a. The laser interferometer alignment ........ 66

b. The uncertainty in measuring $\Delta z_{L} \ldots \ldots 6$

c. The variation of $z_{L}$ for a freely-swinging balance beam . . 67

d. The variation of $\mathrm{C}$ for a freely-swinging balance beam . . . 67

e. The variation of $z_{L}$ and $C$ for a servoed balance beam .... 67

f. The electrode shape-dependence of C . . . . . . 68

APPENDIX D. VARIATIONS OF $C$ WITH TEMPERATURE . . . . . 71

a. Variation of $\mathrm{C}$ with uniform temperature changes ...... 71

b. Variation of $\mathrm{C}$ as the carriage moves through a vertical

temperature gradient ............. 72

c. Variation of $C$ for a radial temperature gradient across

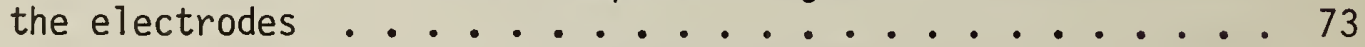

APPENDIX E. DERIVATION OF THE FORCE EQUATIONS . . . . . . 74

APPENDIX F. COORDINATES OF POINTS UNDERGOING ONLY $\gamma_{y}$ and $\beta_{y}$ MOTIONS
MOTIONS

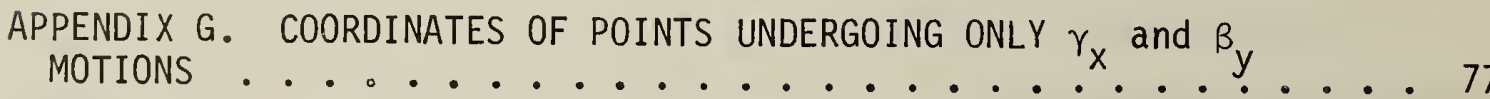

APPENDIX H. PROOF THAT $h_{x}^{I}=h_{x}^{0}$ and $h_{y}^{I}=h_{y}^{0} \ldots \ldots . . . .79$

APPENDIX I. GENERALIZED TORQUES FOR $\gamma_{y}$ AND $\beta_{y}$ MOTIONS ONLY . . . 81

a. Derivation of $Q_{\gamma_{y}} \ldots \ldots \ldots 1$

b. Derivation of $Q_{\beta_{y}} \ldots \ldots \ldots \ldots 2$

APPENDIX J. GENERALIZED TORQUES FOR $\gamma_{x}$ AND $\beta_{y}$ MOTIONS ONLY . . 84

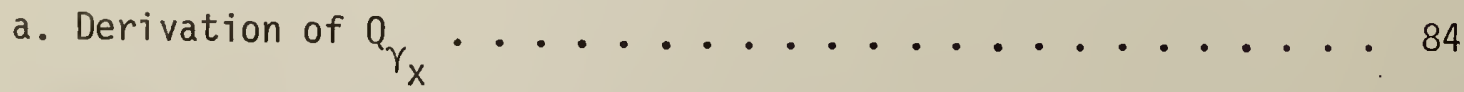

b. Derivation of $Q_{B_{y}} \ldots \ldots \ldots . \ldots \ldots$ 
$\underline{\text { Page }}$

APPENDIX K. BALANCE BEAM STIFFNESS AND SENSITIVITY . . . . . . 86

APPENDIX L. KINETIC ENERGY FOR $\gamma_{y}$ AND $\beta_{y}$ MOTIONS ONLY . . . . . 89

APPENDIX M. KINETIC ENERGY FOR $\gamma_{X}$ AND $\beta_{y}$ MOTIONS ONLY . . . . . 90

APPENDIX N. D'ALEMBERT'S PRINCIPLE FOR $\gamma_{y}$ AND $\beta_{y}$ MOTIONS ONLY $\quad . \quad 91$

a. Equation of motion for the suspended electrode system . . . . 91

b. Equation of motion for the balance beam . . . . . . 92

APPENDIX 0. D'ALEMBERT'S PRINCIPLE FOR $\gamma_{x}$ AND $\beta_{y}$ MOTIONS ONLY $\cdots 95$

a. Equation of motion for the suspended electrode system . . . 95

b. Equation of motion for the balance beam ........ . 96

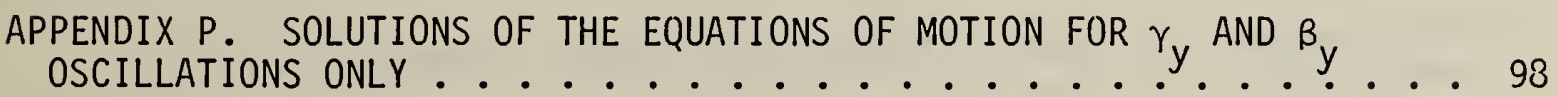

a. The suspended electrode motion ........... 98

b. The equilibrium angle $\hat{\gamma}_{y} \ldots \ldots 100$

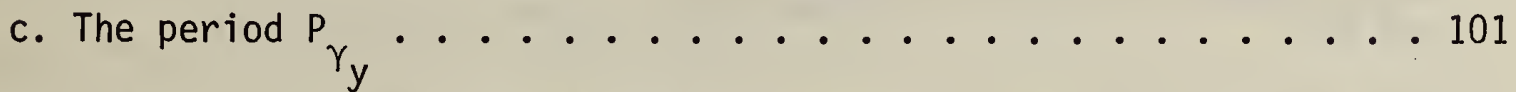

d. The amplitude modulations of $\gamma_{y}$. . . . . . . . 102

e. The balance beam motion ............... 103

f. The equilibrium angle $\hat{\beta}_{y}$. . . . . . . . . . 104

g. The force determination ............. 106

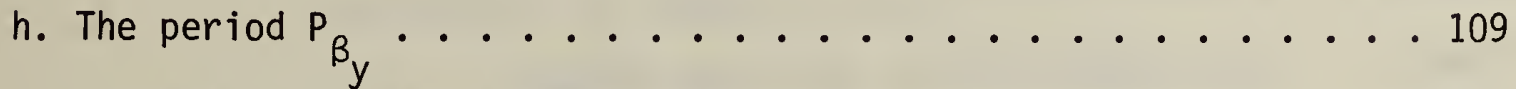

i. The amplitude modulations of $\beta_{y}$. . . . . . . . 110

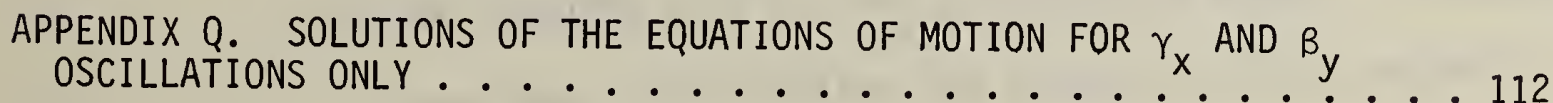

a. The suspended electrode motion . . . . . . . . . 112

b. The equilibrium angle $\hat{\gamma}_{x} \ldots \ldots 113$

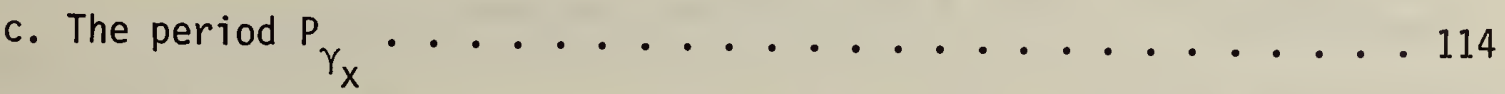

d. The balance beam motion .................. 114

e. The equilibrium angle $\hat{\beta}_{y}^{\prime} \ldots \ldots 116$ 
f. The force determination ............. 117

g. The period $\mathrm{P}_{\beta}^{\prime} \ldots \ldots \ldots \ldots . \ldots . \ldots 118$

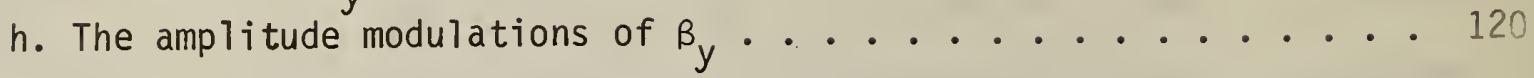

APPENDIX R. DYNAMICAL FORCES ON THE SUSPENDED ELECTRODE AS DETECTED

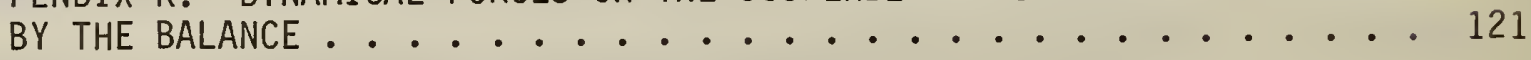

APPENDIX S. DYAMICAL CAPACITANCE VARIATIONS . . . . . . 128

APPENDIX T. ERROR CALCULATIONS FOR THE SLOPE METHOD . . . . . 131

a. Method used to estimate the voltage ratio uncertainty . . . . 131

b. Calculations for Type I scans with a brass electrode at V=9 kV . 132

c. Calculations for Type I scans with an aluminum electrode at

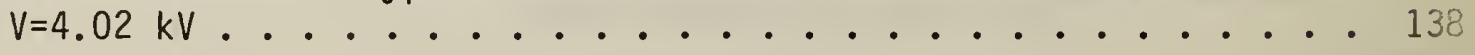

d. Calculations for Type II scans with a brass electrode at $V=9 \mathrm{kV}$ and $\theta_{y}=5 "$. . . . . . . . . . . . . 141

e. Calculations for Type II scans with an aluminum electrode at $V=4.02 \mathrm{kV}$ and $\theta_{y}=5 "$.

f. Calculations for Type III scans with a brass electrode at

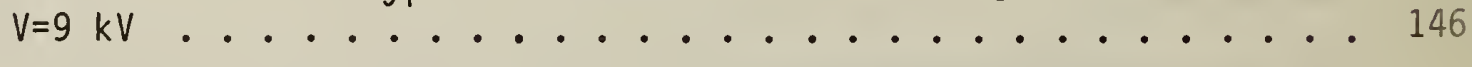

g. Calculations of Type III scans with an aluminum electrode at $\mathrm{V}=4.02 \mathrm{kV}$.

APPENDIX U. DERIVATION OF THE PATH INTEGRAL EQUATION . . . . . 152

APPENDIX V. THE ADJUSTMENT OF F TO $\mathrm{F}^{\prime}$ IN THE PATH INTEGRAL . . . 155

APPENDIX W. A CHECK THAT THE PATH INTEGRAL IS CONSERVATIVE . . . 156

APPENDIX X. THE UNKNOWN PART OF THE PATH INTEGRAL . . . . . . 159

APPENDIX Y. ERROR CALCULATIONS FOR THE PATH INTEGRAL METHOD . . . 161

a. Method used to estimate the voltage ratio uncertainty ..... 161

b. Calculations for Type I scans with a brass electrode at

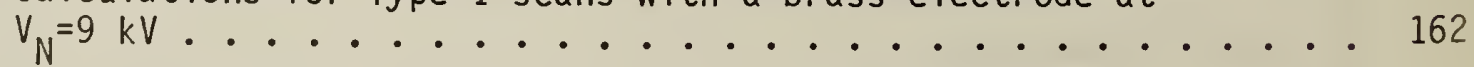

c. Calculation for a Type I scan with an aluminum electrode at

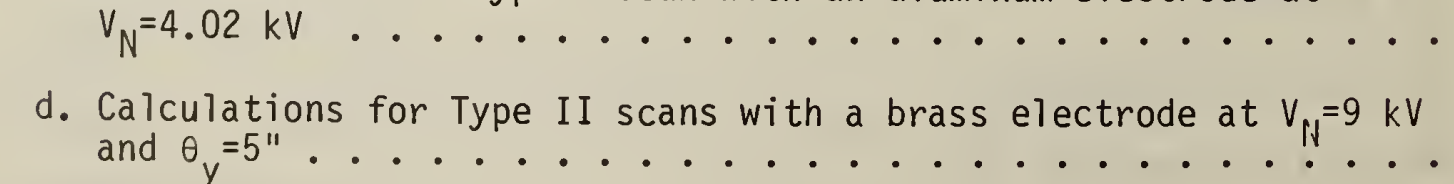


e. Calculations for Type II scans with an aluminum electrode at $V_{N}=4.02 \mathrm{kV}$ and $\mathrm{e}_{\mathrm{y}}=5 " \ldots \ldots . . \ldots 170$

f. Calculations for Type III scans with a brass electrode at $V_{N}=9 \mathrm{kV}$

g. Calculations for Type III scans with an aluminum electrode at $V_{N}=4.02 \mathrm{kV}$

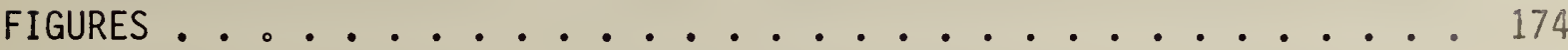

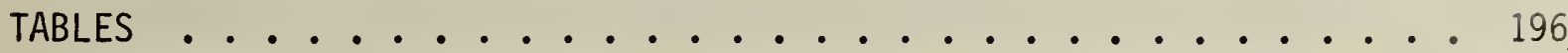



An analysis of the NBS SI volt experiment has been made first to obtain algebraic expressions for the electrical forces present on the suspended electrode of the electrometer. These forces, and the gravitational forces, are used in the Principle of Virtual Work and D'Alembert's Principle to obtain the second order, non-linear, inhomogenous, coupled differential equations of motion for the balance. Exact, analytical solutions of these equations of motion are obtained using small angle approximations and perturbation methods. Estimates are then made of the uncertainties that might result in both the slope and path integral methods in order to determine what requirements must be satisfied to reduce the systematic and random errors of the force determination and the capacitance measurements to acceptable levels so that the SI volt can be determined to within a few ppm.

The mutual alignment of the four electrode surfaces is critical in reducing the size of the horizontal forces between the electrodes. They must be oriented at some position and then maintained over at least a $2.5 \mathrm{~cm}$ carriage scan such that the relative electrode tilts and displacements vary by no more than a few seconds of arc $(20 \mu \mathrm{rad})$ and a few micrometers from perfect alignment in each of two orthogonal directions.

A brass suspended electrode remains in stable equilibrium at $V=9 \mathrm{kV}$, with a resulting vertical electrical force that is equivalent to a gravitational force due to a 5 gram mass $(m)$. The present aluminum electrode could only be used at $V=4.02 \mathrm{kV}(\mathrm{m}=1 \mathrm{gram})$. 
The suspended electrode will execute simple harmonic motion about an equilibrium angle, and the amplitude of this motion needs to be less than 6" (30 $\mu \mathrm{rad})$ during both the force and the capacitance measurements. The balance beam will also execute simple harmonic motion about its equilibrium angle. This equilibrium angle should be such that the plane defined by the balance beam knife edges is determined to be within 16" ( $80 \mu \mathrm{rad})$ of gravitational horizon when the masses of both sides of the balance are matched to within $150 \mu \mathrm{g}$. These conditions are for the aluminum suspended electrode at $V=4.02 \mathrm{kV}$. They are 7" (35 $\mu \mathrm{rad}$ ) and $65 \mu \mathrm{g}$, respectively, for a brass electrode at $\mathrm{V}=9 \mathrm{kV}$.

The capacitance changes by 1 ppm if the electrode overlap changes by $0.04 \mu \mathrm{m}$. Therefore, the balance beam cannot be allowed to swing during the capacitance measurements; it must either be mechanically braked or electronically servoed. The errors involved in measuring the electrode overlap are much smaller if the upper part of the laser interferometer is attached to the suspended electrode system. The average temperature, and the radial and vertical temperature gradients need to be maintained to within a few millidegrees Celsius during the measurements.

The capacitance values are very sensitive to the electrode shapes; so there may be large curvatures and changes in shape of the capacitance versus electrode overlap plots. Thus, the slope might not be determined with sufficient accuracy to use the slope method.

The forces are measured at high voltages; so, in the presence of horizontal forces, the suspended electrode equilibrium angle becomes greater - resulting in capacitance values that are actually larger than 
those measured at the low voltages of the capacitance bridge. Also, the contribution of the horizontal components of the path integral are not measured in the present experiment, and thus they contribute significantly to the uncertainty. It is estimated that the uncertainty in comparing the SI volt value with the NBS volt value might be $\pm 4 \mathrm{ppm}$. Experience gained with actual data could increase or decrease this uncertainty estimate by several ppm. 


\section{INTRODUCTION}

1.1 Objectives and order of the analysis

The motivation for this work is first to determine what static and dynamic external forces could be present on the suspended electrode of the cylindrical capacitor used in the NBS SI volt experiment of Dr. F. K. Harris. (This experiment is briefly explained in Section 1.2.) Then, to examine how the presence of these forces will affect the force determination measurements of the balance, the capacitance measurements, and the path integrals, and to determine what requirements must be satisfied in order to reduce the resulting systematic errors in these measurements to acceptable levels.

The first step towards achieving these goals is presented in Section 2, in which an algebraic equation is developed that adequately predicts the capacitance values as a function of the electrode overlap, the electrode tilts, and the relative electrode radial displacement. The electrical forces between the electrodes are then obtained from partial derivatives of this capacitance equation. These forces are used in the Principle of Virtual Work and D'Alembert's Principle $[1,2]$ in Section 3 to obtain the equations of motion for the balance. Analytical solutions of these second order, non-linear, inhomogeneous, coupled differential equations are obtained using small angle approximations and perturbation methods. Systematic errors in the balance measurements due to unwanted electrode forces immediately follow from the solutions to these equations of motion. The 
electrical forces are integrated over possible paths of the moveable electrodes in Section 4 to obtain an algebraic expression for the electrical work done by the system. This path integral equation is equal to the change in energy stored in the electric field between the capacitors, which is a function of the voltage applied to the electrodes. Systematic errors in the force determination measurements and in the capacitance measurements are then incorporated in path integral calculations to estimate the effects of these errors in the voltage determination. Section 5 then summarizes the results of this work.

This paper pertains to a particular experiment. However, the algebra in Section 3 can be applied to any balance measurement in which horizontal forces are present on the object to be weighed; the object may be at rest or swinging.

Liberal use is made of appendices so that the sections wịll be more straightforward and less cluttered with derivations and equations. The figures and tables are grouped together because many of them are continually referred to in the text.

SI units are used throughout the paper; but, to avoid entering powers of ten into numerical calculations, and to readily comprehend the magnitudes of physical quantities present in the volt experiment, multiples and submultiples of SI units are employed. As a result, an equation may contain lengths expressed in $\mathrm{cm}$ and relative displacements in $\mu \mathrm{m}$. The multiples are clearly stated in the equations, so they can be readily transformed to whatever multiples the reader finds appropriate. 
1.2 Description of the SI volt experiment

The objective of this research is to realize the SI volt in terms of the units of mass, length, time $(\mathrm{kg}, \mathrm{m}, \mathrm{s})$ and the assigned magnetic constant $\left(4 \pi \times 10^{-7} \mathrm{H} / \mathrm{m}\right)$ to an uncertainty of 1 to 3 parts in $10^{6}$ - or equivalently, to determine the NBS volt in terms of the SI volt to this accuracy. This experiment requires a considerable effort in accurate and precise electrical and dimensional metrology.

The present assignment of the volt in terms of the SI ampere and ohm determinations is uncertain by more than $5 \mathrm{ppm}$ [3]. A direct determination of the volt should not have the same systematic errors as those present in the SI ampere experiments - and, since no electrical energy is converted to heat as in the latter experiments, it may be possible to achieve smaller random uncertainties. An improvement in the assignment of the SI volt could provide an absolute value of $2 \mathrm{e} / \mathrm{h}$, which would be useful for Josephson effect volt-maintenance applications throughout the world. It also might resolve the inconsistencies present among the measured values of a number of fundamental constants: for example, the Avogadro and Faraday constants [3].

The method uses a cylindrical electrode suspended from a balance beam as shown in Figure 1. The suspended electrode occupies the annular space between two coaxial cylinders that form the high voltage electrodes. When voltage is applied between the concentric electrodes and the grounded suspended electrode there is an electrical force on the suspended electrode that acts vertically downward in the $z$ 
direction. This force, $F_{Z}$, results from fringing of the radial electric field lines at the lower rim of the suspended electrode, and for the NBS apparatus has a value of $4.91 \times 10^{-2} \mathrm{~N}$ at $9 \mathrm{kV}$. (There is also a net force $-F_{z}$ acting upward at the upper rims of the high voltage electrodes which need not be considered.) This electrical force has the same magnitude as that of a gravitational force due to increasing the weight of the suspension system by the addition of a mass $m$ of 5 grams to a weight holder with voltage-off. The electrical force, $F_{z}$, can therefore be compared with a known weight, $m g$, by a substitution procedure where $g$ is the local value of the acceleration of gravity at the weight holder.

The 2-knife balance beam generally follows the design of the NBS No. 2 kilogram comparator [4] - except that the inter-knife separation has been increased to $7.5 \mathrm{~cm}$. The suspension knife shown in Figure 1 actually consists of a load knife and an intermediate knife crossed in the same horizontal plane; so the suspension system is free to pivat with the same lever arm about any direction. A resolution and stability of $1 \mathrm{\mu g}$ has been achieved by $\mathrm{Dr}$. Harris with this balance for voltage-off when using an.aluminum electrode with a resulting suspension system mass of $2.35 \mathrm{~kg}$.

The two concentric cylinders (henceforth referred to as the carriagt electrodes) rest upon an electrically insulated stand on a moveable carriage that can be raised or lowered by a decoupled elevating shaft. Vertical alignment of the carriage is maintained by means of teflon bearings riding on three stainless steel guide rods positioned in a ring spaced $120^{\circ}$ apart. The carriage displacement, $\Delta z$, is monitored 
by a laser interferometer system having a reproducibility of $\pm 0.025 \mu \mathrm{m}$.

The entire apparatus is enclosed in an 0-ring sealed container and will be maintained with an overpressure in an environment of either dry air or sulfur hexafluoride. The container is surrounded by thermal insulating material to minimize temperature fluctuation effects.

There are two ways of obtaining the SI volt - the slope method and the path integral method. In the slope method the electrical force at any point $z$ is

$$
F_{z}=m g=-(1 / 2) v^{2}(\partial C / \partial z)
$$

where $C$ is the capacitance, $\partial \mathrm{C} / \partial \mathrm{z}=12.12 \times 10^{-10} \mathrm{~F} / \mathrm{m}(12.12 \mathrm{pF} / \mathrm{cm})$, and at $V=9,000 \mathrm{~V}, F_{Z}=4.91 \times 10^{-2} \mathrm{~N}$ and $F_{z} / g=m=0.005 \mathrm{~kg}$. Therefore, $V$ can be obtained by measuring $m, g, F_{z}$ and $\partial C / \partial z$ at point z. In the path integral method the electrical work required to move the electrodes equals the change of electrical potential energy stored in the field between the electrodes; in an ideal situation

$$
\int_{z_{i}}^{z_{f}} F_{z} d z=\int_{z_{i}}^{z_{f}} \operatorname{mgdz}=(1 / 2) v^{2}\left(C_{f}-C_{j}\right)
$$

and $V$ is obtained from the path integral and $C_{j}$ and $C_{f}$. The three terminal capacitance value, $C$, is measured at 100 volts a.c. in both methods with a transformer ratio-arm bridge [5] having a resolution of 
one part in $10^{8}$. The capacitance has been previously related to the SI unit of length by using a computable cross-capacitor [6].

The SI value of the applied voltage can thus be determined in both methods from the measured quantities. (The same quantities are used in both methods, but in different ways.) The comparison between the SI value of the volt and the NBS legal volt, maintained at the one-volt level via the Josephson effect, will be made using a resistance voltage divider whose ratio uncertainty is $0.2 \mathrm{ppm}$ [7] and a group of calibrated standard cells.

The above equations are for perfect right circular cylinders exactly centered, and aligned parallel to the gravitational field. If these conditions are not met then $F_{z}$ is not a constant at constant voltage and $\partial C / \partial z$ also is not a constant. The analysis will usually assume that the electrodes are right cylinders, but not necessarily perfect, and will study the effects of electrode misalignments. Arguments will be given that the path integral method may be the more promising one.

The electrodes are gold plated to provide an inert, oxide-free surface. There may be migrating dielectric films on the electrode surfaces that could affect the 100 volt a.c. capacitance measurements, which in turn might be different from the assumed same capacitance values for the kilovolt d.c. force measurements. There could also be corona discharge from dust on the electrode surfaces, and there may be resonances in the capacitance values for capacitance measurements using frequencies near a mechanical resonance of either the electrodes or their support system [8]. The possible problems of this paragraph will not be addressed in this paper. 


\section{CAPACITANCE AND ELECTRICAL FORCE EQUATIONS}

2.1 Coordinate system for the balance

There are two coordinate systems used in the calculations. The one for the balance is presented in this section, while the one used to completely describe the combined relative electrode orientations is given in Section 2.3. Figure 2 shows the balance coordinate system, with the origin located at the center knife position $(C)$. The $z$ axis, which is parallel to the gravitational field, points downward so that increasing vertical electrode separations correspond to increasing laser interferometer readings. The $y$ axis is parallel to the direction that the balance beam would have if the plane defined by the two knife edges at contact points $(P)$ and $(C)$ were at gravitational horizon. The $x$ axis is directed into the figure so that the coordinate system will be right-handed. Cartesian coordinates are used because the cylindrical symmetries are broken by the balance beam.

Point masses are shown for the suspension system, the balance beam and the counterweight; they will be given distributed masses when required. Equations for the electrical forces $F_{z}, F_{y}^{I}$ and $F_{y}^{0}$ will be derived in Section 2.4. The superscripts, I and 0 , refer to the inner and outer electrode surfaces respectively.

Normally a gimbal would replace the connecting ring. shown in Figure 1: but, the equations of motion would be unneccessarily complicated for that case. Therefore the equations of motion in Section 3 involve a single, eliptical pendulum rather than a double pendulum. This simplification will not affect the final conclusions. 
Each electrode surface has an electrical symmetry axis, which is a straight line for a right cylinder or a conical section. Figure 2 shows the combined symmetry axis of the four electrode surfaces when they are all aligned parallel to the $z$ axis and when $B_{y}$ equals zero. If any electrode surface significantly deviates from radial symmetry then the combined symmetry axis is a curved, three-dimensional line.

The carriage electrodes can be displaced from the symmetry axis by the distances $d_{x}$ and $d_{y}$, and tilted about the symmetry axis by $\theta_{x}$ and $\theta_{y}$. The suspended electrode can be displaced from the symmetry axis by $D_{y}$ as a result of the balance beam being at an angle $B_{y}$ from gravitational horizon. The suspended electrode can also tilt by the angles $\gamma_{x}$ and $\gamma_{y}$. Note that the quantities $\beta_{y}, \gamma_{x}, \gamma_{y}, D_{y}, \theta_{x}, \theta_{y}$, $d_{x}, d_{y}, \ell_{b}$ and $\ell_{B}$ all have signs associated with them, and of those shown in Figure 2, all are given positive signs except for $d_{y}$ - which is negative there - because carriage electrode displacements have the opposite effect of suspended electrode displacements. The displacements and tilts are greatly exaggerated in this figure, which is also not drawn to scale.

Table 1 lists the length and mass values of quantities shown in Figure 2, assuming either an aluminum or a brass suspended electrode. The value of the electrode overlap for $\theta_{x}, \theta_{y}, \gamma_{x}$ and $\gamma_{y}$ equal to zero $\left(L_{0}=L_{0}^{I}=L_{0}^{0}\right)$ is assumed to be $L_{0}=10 \mathrm{~cm}$ at $C=100 \mathrm{pF}$. Measurements have not been made of this value, but it is actually quite close to 10 $\mathrm{cm}$ - and the calculations are insensitive to changes in $L_{0}$. Note that the carriage position is indirectly given in this coordinate system via $L_{0}^{I}$ and $L_{0}^{0}$. 
The effects that the knives at points (C) and (P) of Figure 2 have on the balance arm lengths $L_{A}$ and $L_{B}$ are shown in Figures 3 and 4 ; they are assumed to have radii of curvature $\rho_{C}$ and $\rho_{p}$, respectively. The best knife edges have radii of $0.25 \mu \mathrm{m}$, but they can be larger [9]. The value $5 \times 10^{-5} \mathrm{~cm}(0.5 \mu \mathrm{m})$ will be used for both knives; also, they are assumed to be symmetrically honed. Note in Figure 3 that the origin of the balance coordinate system moves in the $\pm y$ direction with the center knife contact point. This choice simplifies the algebra, but the coordinate system is not an inertial frame of reference; however, since the center knife horizontal accelerations are so small, it is essentially an inertial frame.

2.2 Capacitance equations for individual electrode displacements or tilts

Equations are derived in Appendices $A$ and $B$ for the capacitance of the electrodes used in the SI volt experiment when either the carriage electrodes or the suspended electrode are displaced from, or tilted about, the combined electrode symmetry axis. The results for individual electrode displacements or tilts are

$$
\begin{aligned}
& c\left(d, z_{L}\right)=c\left(z_{L}\right)\left[1+\left(6.5 \times 10^{-9}\right) d^{2}\right] \\
& c\left(D_{y}, z_{L}\right)=c\left(z_{L}\right)\left[1+\left(6.5 \times 10^{-9}\right) D_{y}^{2}\right] \\
& c\left(\theta, z_{L}\right)=c\left(z_{L}\right)\left[1+\left(6.5 \times 10^{-9}\right) d_{t}^{2}\right] \\
& c\left(\gamma, z_{L}\right)=c\left(z_{L}\right)\left[1+\left(6.5 \times 10^{-9}\right) d_{T}^{2}\right]
\end{aligned}
$$


where

$$
\begin{aligned}
& C\left(z_{L}\right)=\left[100 p F-(12.12 p F / c m) z_{L}\right] \\
& D_{y}=10^{4}\left(L_{A} / 2\right) \beta_{y}^{2} \\
& d_{t}=10^{4}\left[\ell-L_{0}\left(z_{L}\right) / 2\right] \theta \\
& d_{T}=10^{4}\left[\left(L-L_{0}\left(z_{L}\right) / 2\right] \gamma\right.
\end{aligned}
$$

[C in pF; $d, D_{y}, d_{t}, d_{T}$ in $\mu \mathrm{m} ; z_{L}, L_{A}, \ell, L, L_{0}$ in $c m ; B_{y}, \theta, \gamma$ in $\mathrm{rad}]$

with $d^{2}=d_{x}^{2}+d_{y}^{2}, \theta^{2}=\theta_{x}^{2}+\theta_{y}^{2}$, and $\gamma^{2}=\gamma_{x}^{2}+\gamma_{y}^{2}$. The quantities are defined in Appendices $A$ and $B$ and in Figures 2 and 5.

\subsection{Coordinate system for the relative electrode positions}

Section 2.2 gave the capacitance equations when either the suspended electrode is displaced or tilted, or the carriage electrodes are displaced or tilted. The problem is how to combine these effects. If, for example, $d_{y}=-D_{y}$ (as defined in Figure 2) then Equations 2-1 and 2-2 would predict that $c \approx C\left(z_{L}\right)\left[1+2\left(6.5 \times 10^{-9}\right) d_{y}^{2}\right]$, when actually $C=C\left(z_{L}\right)$ because all the electrodes have been translated in the positive $y$ direction by the same amount $D_{y}$. If, on the other hand, $d_{y}=D_{y}$ then Equations 2-1 and 2-2 would again predict that $C \approx$ $C\left(z_{L}\right)\left[1+2\left(6.5 \times 10^{-9}\right) d_{y}^{2}\right]$, when actual1y $c=c\left(z_{L}\right)[1+4(6.5 \times$ $\left.10^{-9}\right) d_{y}^{2}$ ] because the electrodes have been radially separated by the 
amount $2 d_{y}$. Clearly, the electrode displacements and tilts must be expressed in relative coordinates.

Figure 6 shows the suspended electrode and the outer carriage electrode each displaced from, and tilted about, the combined electrode symmetry axis. It is shown in Appendix B that the effective displacements due to electrode tilts, $d_{t}$ and $d_{T}$, can be vertically located at half the electrode overlap - the same as for $d_{y}$ and $D_{y}$. We can therefore add these quantities to obtain the total relative horizontal displacements, $h_{x}^{0}$ and $h_{y}^{0}$, of the electrodes from the combined symmetry axis:

$$
\begin{aligned}
h_{x}^{0}= & 10^{4}\left[L-L_{0}\left(z_{L}\right) / 2\right] \gamma_{x}+10^{4}\left[\ell-L_{0}\left(z_{L}\right) / 2\right] \theta_{x}+d_{x} \\
h_{y}^{0}= & 10^{4}\left(L_{A} / 2\right) \beta_{y}^{2}+10^{4}\left[L-L_{0}\left(z_{L}\right) / 2\right] \gamma_{y}+ \\
& 10^{4}\left[l-L_{0}\left(z_{L}\right) / 2\right] \theta_{y}+d_{y}
\end{aligned}
$$

[h, d in $\mu m ; L, \ell, L_{0}, L_{A}, z_{L}$ in $\mathrm{cm} ; \beta, \gamma, \theta$ in $\left.\mathrm{rad}\right]$.

Note that the angles $\beta, \gamma$ and $\theta$ all have signs associated with them as defined in Figures 2 and 6 ; so the signs of individual displacements are automatically accounted for.

If the inner and outer surfaces of the suspended electrode and the carriage electrodes are parallel, then it is clear from the form of Equations 2-9 and 2-10 that

$$
h_{x}^{0}=h_{x}^{I}=h_{x} \text { and } h_{y}^{0}=h_{y}^{I}=h_{y}
$$


These equalities will be more rigorously derived in Appendix $H$.

We now have a coordinate system, shown in Figure 7 , that completely describes the relative orientations of the electrodes in terms of the relative coordinates $h_{x}, h_{y}$ and $z_{L}$, where $z_{L}$ is the lase interferometer reading, $z_{\ell}$, relative to the initial value $z_{j}$ at $C=$ $100 \mathrm{pF}$ and $h_{x}=h_{y}=0$. This coordinate systern should not be thought of as being attached to any object in Figure 1 ; all the coordinates in Figure 7 are relative quantities derived from Equations 2-9 and 2-10, and from the relationship $z_{L}=z_{\ell}-z_{j}$. The forces shown in Figure 7 will be derived in Section 2.5.

\subsection{The capacitance equation}

The capacitance, as a function of $h_{x}, h_{y}$ and $z_{L}$, immediately follows from Equations 2-1, 2-9, and 2-10:

$$
\begin{aligned}
& c\left(h, z_{L}\right)=c\left(z_{L}\right)\left[1+\left(6.5 \times 10^{-9}\right) h^{2}\right] \\
& c\left(z_{L}\right)=\left[100 p F-(12.12 p F / c m) z_{L}\right]
\end{aligned}
$$

[C in $\mathrm{pF} ; \mathrm{h}$ in $\mu \mathrm{m} ; \mathrm{z}_{\mathrm{L}}$ in $\mathrm{cm}$ ]

where $h^{2}=h_{x}^{2}+h_{y}^{2}$.

This equation ignores any effects due to the rounded electrode rims that might not be included in the $100 \mathrm{pF}$ calibration point at $L_{0}=$ $10 \mathrm{~cm}$. It al so assumes that the suspended electrode does not 
significantly interact with the horizontal surface located at the bottom of the carriage electrodes. All that will be required for this paper is to predict the magnitude of the capacitance to within $10-20 \%$ of the actual value, so that the effects of dynamical forces can be studied. It is the capacitance variations that will be of concern - not the absolute values.

Appendix $C$ demonstrates the sensitivity of the $C\left(h, z_{L}\right)$ values to changes in $z_{L}$. If the electrode overlap changes by $\pm 0.041 \mu \mathrm{m}$ when $C \approx 50 \mathrm{pF}$, then the value of $\mathrm{C}\left(\mathrm{h}, \mathrm{z}_{\mathrm{L}}\right)$ changes by $\pm 1 \mathrm{ppm}$. So the balance beam cannot be allowed to swing during capacitance measurements. This appendix also shows that the capacitance values are very sensitive to the shapes of the electrode surfaces. The calculations of Appendix $D$ further suggest that the temperature distribution inside the 0 -ring sealed container must be maintained to within a few millidegrees Celsius during all of the data collection periods in order to keep the resulting capacitance variations below the $1 \mathrm{ppm}$ level.

\subsection{The force equations}

The equations for the $h_{x}, h_{y}$ and $z_{L}$ components of the electrical force are derived in Appendix E. Using Equations E-3 to E-5 and Equations 2-12 and 2-13, and converting the quantities to similar dimensions yields the results

$$
F_{h_{x}}\left(h, z_{L}\right)=\left(3.25 \times 10^{-9}\right) v^{2}\left(h, z_{L}\right) C\left(z_{L}\right) h_{x}
$$




$$
\begin{aligned}
& F_{h_{y}}\left(h, z_{L}\right)=\left(3.25 \times 10^{-9}\right) v^{2}\left(h, z_{L}\right) C\left(z_{L}\right) h_{y} \\
& F_{z_{L}}\left(h, z_{L}\right)=-\left(6.06 \times 10^{-4}\right) v^{2}\left(h, z_{L}\right)\left[1+\left(6.5 \times 10^{-9}\right) h^{2}\right] \\
& C\left(z_{L}\right)=\left[100 p F-(12.12 p F / c m) z_{L}\right]
\end{aligned}
$$

[F in $N$; $V$ in $k V ; C$ in $p F ; h$ in $\mu \mathrm{m} ; z_{L}$ in $\mathrm{cm}$ ]

where $n^{2}=n_{x}^{2}+n_{y}^{2}$. These are the equations for the forces expressed in the relative coordinate system of Figure 7; they will be used in evaluating the path integrals in Section 4.

In order to solve the equations of motion in Section 3 we will need expressions for the electrical forces which act on the suspended electrode. Referring to Figure 2, they are:

$$
\begin{aligned}
& F_{x}^{0}=F_{x}^{I}=F_{h_{x}}\left(h, z_{L}\right) \\
& F_{y}^{0}=F_{y}^{I}=F_{h_{y}}\left(h, z_{L}\right) \\
& F_{z}=-F_{z_{L}}\left(h, z_{L}\right)
\end{aligned}
$$

where $F_{x, y}^{0}=F_{x, y}^{I}$ because $h_{x, y}^{0}=h_{x, y}^{I}$ and $c^{0}\left(z_{L}\right)=c^{I}\left(z_{L}\right)$. Note that these are the net forces which remain after sumiming the incremental forces over the surfaces of the suspended electrode.

The vertical force $F_{z}$ is placed at point 4 in Figure 2 at the center axis of the suspended electrode rim since the vertical forces act primarily at the rim. $F_{y}^{0}$ and $F_{y}^{I}$ are placed at points 5 and 6 , respectively, at one half the overlap distance of each electrode because this was found in Appendix $B$ to be the centroid locations of 
the inner and outer capacitance contributions. $F_{x}^{0}$ and $F_{x}^{I}$ are placed at analogous points in the $x-z$ plane.

We can now express the vertical electrical force $F_{Z}$ in terms of the independent variables $\gamma_{x}, \gamma_{y}$ and $\beta_{y}$ of Figure 2 by using Equations $2-16,2-19,2-9,2-10$ and 2-11. We will next solve the equations of motion in Section 3 to see if the dynamical balance measurements of $F_{z}$ are affected by the presence of the horizontal forces. 


\subsection{Generalized torques}

The equations of motion for the balance and suspended electrode system will be obtained using the Principle of Virtual Work and D'Alembert's Principle $[1,2]$. The first step is to determine the $\left(x_{i}, y_{i}, z_{j}\right)$ coordinates of the points in Figure 2 which locate the positions of the gravitational and electrical forces $M_{A} g, M_{b} g, M_{B} g$, $F_{z}, F_{x}^{0}, F_{x}^{I}, F_{y}^{0}$ and $F_{y^{*}}^{I}$. These coordinates are listed (using Figures 2, $3,4,8$ and 9 ) in Appendix $F$ for $\gamma_{y}$ and $B_{y}$ motions only, and in Appendix $G$ for $\gamma_{x}$ and $B_{y}$ motions only.

In general there would be simultaneous $\gamma_{x}, \gamma_{y}$ and $\beta_{y}$ motions for particular values of $d_{x}, d_{y}, \theta_{x}$ and $\theta_{y}$ - and the variable $\gamma$ would be more appropriate than $\gamma_{x}$ and $\gamma_{y}$. However, an expression such as $y_{5}$ in Appendix $F$ would be much more complicated, since one would have to trace from point 5 around the outer suspended electrode surface until it intersected the $y-z$ plane at $x=0$. To avoid this solid geometry problem, we will assume special cases in which only $F_{x}^{I}$ and $F_{x}^{0}$ or $F_{y}^{I}$ and $F_{y}^{0}$ are present, and will ignore the $D_{y}$ displacements when considering the $\gamma_{x}$ and $\beta_{y}$ motions. These special cases are similar to the normal mode motions, and should adequately cover the physics of the real system.

Appendix $H$ uses Figure 2 and coordinates in Appendices $F$ and $G$ to more riguorously prove that $h_{x}^{0}=h_{x}^{I}=h_{x}$ and that $h_{y}^{0}=h_{y}^{I}=h_{y}$. It then follows from Equations 2-14, 2-15, 2-17 and 2-18 that $F_{X}^{0}=F_{X}^{I}$, and that $F_{y}^{0}=F_{y^{\circ}}^{I}$. The forces, and their locations, are thus known in 
terms of either the independent variables $\gamma_{y}$ and $\beta_{y}$ or the variables $\gamma_{x}$ and $\beta_{y}$. We will next use this information in the Principle of Virtual Work to obtain the generalized torques.

The Principle of Virtual Work states that:

$$
\delta W=\sum_{i=1}^{6}\left[\left(F_{i}\right)_{x} \delta x_{i}+\left(F_{i}\right)_{y} \delta y_{i}+\left(F_{i}\right)_{z} \delta z_{j}\right]
$$

where for $\gamma_{y}$ and $\beta_{y}$ motions $x_{i}=x_{j}\left(\gamma_{y}, \beta_{y}\right), y_{i}=y_{i}\left(\gamma_{y}, \beta_{y}\right), z_{i}=$ $z_{i}\left(\gamma_{y}, \beta_{y}\right)$, and the index $i$ represents any of the six points shown in Figure 2; so

$$
\delta x_{i}=\left(\partial x_{j} / \partial \gamma_{y}\right) \delta \gamma_{y}+\left(\partial x_{i} / \partial \beta_{y}\right) \delta \beta_{y} \text {, etc. }
$$

Substituting Equation 3-2 into Equation 3-1 yields

$$
\delta W=\begin{aligned}
& \sum_{i=1}^{6}\left[\left(F_{i}\right)_{x}\left(\delta x_{i} / \delta \gamma_{y}\right)+\left(F_{i}\right)_{y}\left(\partial y_{i} / \partial \gamma_{y}\right)+\left(F_{i}\right)_{z}\left(\partial z_{i} / \partial \gamma_{y}\right)\right] \delta \gamma_{y}+ \\
& \sum_{i=1}^{6}\left[\left(F_{i}\right)_{x}\left(\partial x_{i} / \partial \beta_{y}\right)+\left(F_{i}\right)_{y}\left(\partial y_{i} / \partial \beta_{y}\right)+\left(F_{i}\right)_{z}\left(\partial z_{i} / \partial \beta_{y}\right)\right] \delta \beta_{y}
\end{aligned}
$$

or

$$
\delta W=Q_{y} \delta \gamma_{y}+Q_{B_{y}} \delta B_{y}
$$

where $Q_{\gamma_{y}}$ and $Q_{B_{y}}$ are the generalized torques. Equations 3-3 and 3-4 can also be used for $\gamma_{x}$ and $\beta_{y}$ motions by replacing $\gamma_{y}$ with $\gamma_{x}$. The generalized torques for $\gamma_{y}$ and $\beta_{y}$, and $\gamma_{x}$ and $\beta_{y}$ motions are given in Appendices $I$ and $J$ respectively. 
We can use the generalized torque equations derived in Appendices $I$ and $J$ to study several properties of the system when both the balance beam and the suspended electrode are in static equilibrium, with equilibrium angles $\hat{\beta}_{y}, \hat{\gamma}_{x}$ and $\hat{\gamma}_{y}$ respectively. For this situation $Q_{\gamma_{x}}=Q_{\gamma_{y}}=Q_{B_{y}}=0$.

Equations I-3 and J-3 can be used to solve for $\hat{\gamma}_{x}$ and $\hat{\gamma}_{y}-$ which have identical forms if $\hat{\beta}_{y}=0$. Values of $\hat{\gamma}_{x}$ and $\hat{\gamma}_{y}$ are listed for various combinations of $d_{x}$ and $\theta_{x}$ or $d_{y}$ and $\theta_{y}$ in Tables 3-7, using either an aluminum cylinder in the suspended electrode system $\left(M_{A}=2.35 \mathrm{~kg}\right)$, or a brass cylinder $\left(M_{A}=5.47 \mathrm{~kg}\right)$. The voltage values for these tables are such that the electrical forces, $F_{Z}$, are equal to the gravitational forces, $\mathrm{mg}$, for integer masses between 1 and 5 grams. The suspended aluminum electrode is in unstable equilibrium for voltages larger than $6.3 \mathrm{kV}$; $\underline{\text { j.e. }}$. it would swing outward until it collided with the carriage electrodes. The aluminum electrode is stable at $V=4.02 \mathrm{kV}$, but the 1 gram mass could only be determined to within $1 \mathrm{ppm}$. It is therefore preferable to use the brass electrode, which is stable at $V=9 \mathrm{kV} . \mathrm{L}_{\mathrm{cm}}$ in this case is $2 \mathrm{~cm}$ below the top of the suspended electrode rather than $6 \mathrm{~cm}$ above the top.

The $\hat{h}_{x}$ and $\hat{h}_{y}$ (i.e. $\hat{h}_{x, y}$ ) values listed in these tables are obtained from Equations 2-9, 2-10 and 2-11; the $2 F_{x, y}^{0}$ values from Equations 2-14, 2-15, 2-17 and 2-18; the $\Delta \hat{F}_{z} / F_{z}$ values from Equations 2-16 and 2-19; and the $\Delta \hat{C} / C\left(z_{L}\right)$ values from Equations 2-12 and 2-13. The $\delta\left(\hat{C} / C\left(z_{L}\right)\right)$ numbers are the differences in the $\Delta \hat{C} / C\left(z_{L}\right)$ values between the appropriate kilovolt values used in the force 
determinations and the $v_{r m s}=100$ volt value used in the a.c. capacitance measurements (in which $\hat{\gamma}_{x}$ and $\hat{\gamma}_{y}$ are essentially zero, so that the $10^{4}\left(L-L_{0} / 2\right) \hat{\gamma}_{x, y}$ term does not contribute to $\hat{h}_{x}$ or $\hat{h}_{y} \cdot \quad \hat{F}_{x}^{0}$ and $\hat{F}_{y}^{0}$ are large fractions of $F_{z}$ because most of the electric field lines are radial; thus, small horizontal displacements produce large horizontal forces.

Autocollimator measurements of $\theta_{x}$ and $\theta_{y}$ each yield smooth variations of several seconds of arc over a carriage travel span of a few centimeters, with a $I^{\prime \prime}$ repeatability. The variations of $d_{x}$ and $d_{y}$ are not presently known. It will be assumed in many of the sample calculations which follow that a brass suspended electrode is used, with $V=9 \mathrm{kV}, d_{x}=d_{y}=0$ and $\theta_{x}$ or $\theta_{y}=5^{\prime \prime}$. This may yield values of $\hat{h}_{x}$ or $\hat{h}_{y}$ that are better or worse than the actual relative displacements, depending upon the sizes of $d_{x}$ and $d_{y}$, but it should provide reasonable information about any potential problems.

Appendix $K$ derives the balance beam sensitivity equations, and shows that there should be no difficulty in obtaining a $1 \mu \mathrm{g}$ resolution when using the brass suspended electrode at $V=9 \mathrm{kV}$. There would, however, be more stresses induced on the balance when converting between the voltage-on and the voltage-off configurations with these larger masses for $M_{A}$ and $M_{B}$. The $\hat{F}_{y}^{0}$ dependency of the balance sensitivity can be avoided if the balance is used as a null device by adjusting the voltage so that $\hat{\beta}_{y}(O N)=\hat{\beta}_{y}(O F F)$.

\subsection{Equations of motion and their solutions}

\section{D'Alembert's Principle states that}




$$
(d / d t)(\partial T / \partial \dot{x})-(\partial T / \partial x)=Q_{X}
$$

where $x$ represents the generalized coordinates $\gamma_{x}, \gamma_{y}$ or $\beta_{y}$. The generalized torques, $Q_{X}$, were found in Appendices $I$ and $J$. The kinetic energy, $T$, is also required - and is evaluated in Appendices $L$ and $M$ for $\gamma_{y}$ and $\beta_{y}$ or $\gamma_{x}$ and $\beta_{y}$ motions, respectively. Equation 3-5 is used in Appendices $N$ and 0 to obtain the equations of motion for small angles; the factor $10^{-2}$ appears on the 1.h.s. of Equations $\mathrm{N}-1$, $\mathrm{N}-4,0-1$ and $0-4$ in order that both sides of the equations will have the dimensions $\mathrm{N} \cdot \mathrm{cm}$. The equations of motion (Equations $\mathrm{N}-2, \mathrm{~N}-5,0-2$ and $0-5)$ are second order, non-1 inear, inhomogenous, coupled differential equations.

Appendices $P$ and $Q$ give the solutions of these equations by use of a perturbation method. The suspended electrode executes simple harmonic motion about the equilibrium angles $\hat{\gamma}_{x}$ and $\hat{\gamma}_{y}$, with very small $\cos 2 \omega_{\beta_{y}} t$ and $\cos \omega_{\beta_{y}} t$ amplitude modulations. The period, $P_{\gamma_{y}}$, of this pendulum-like motion is voltage-dependent, and as is shown in Figure 10 for a brass suspended electrode, increases from a value of $1.48 \mathrm{~s}$ at $\mathrm{V}=0 \mathrm{kV}$ to a value of $2.82 \mathrm{~s}$ at $\mathrm{V}=9 \mathrm{kV}$.

The balance beam also executes simple harmonic motion about the equilibrium angle $\hat{\beta}_{y}$, with very small $\cos 2 \omega_{\gamma_{y}} t$ and $\cos \omega_{\gamma_{y}} t$ amplitude modulations. The period, $P_{\beta_{y}}$, of this motion is also voltage-dependent, and for a balance sensitivity of $0.11 \% / \mu g$ increases from a value of $44.9 \mathrm{~s}$ at $V=0 \mathrm{kV}$ to $46.8 \mathrm{~s}$ at $V=9 \mathrm{kV}$ for a brass electrode. Since the pendulum and balance beam periods are so different, only very small energy transfers occur between these 
motions. Hence, the $\tilde{\gamma}$ and $\tilde{\beta}_{y}$ amplitudes do not alternately, and periodically, increase and decrease with time.

The equilibrium angle $\hat{\beta}_{y}$ depends upon $\hat{F}_{z}$, and therefore upon $\hat{\gamma}_{x}$ and $\hat{\gamma}_{y}$ for voltage-on measurements. There is a dynamical systematic error, $\delta \hat{\beta}_{y}$, in $\hat{\beta}_{y}$ which depends upon the voltage, the electrode overlap, and upon $\tilde{\gamma}^{2}=\tilde{\gamma}_{x}^{2}+\tilde{\gamma}_{y}^{2}$. Figure 11 shows a plot of $\delta_{B_{y}}{ }^{(O N)}$ versus $\tilde{\gamma}_{y}$ for a brass suspended electrode at $V=9 \mathrm{kV}$ and $\tilde{\gamma}_{x}=$ 0 ; $\tilde{\gamma}$ must be less than $2.5^{\prime \prime}$ in order to measure $m$, the mass equivalent of $\hat{F}_{z}$, to within $1 \mu \mathrm{g}$ at $V=9 \mathrm{kV}$. This systematic error is zero for voltage-off measurements.

$\hat{F}_{z}$ is measured by first adding mass $m$ to the weight holder and determining $\hat{\beta}_{y}(O F F)$ for voltage-off. Mass $m$ is then removed and the voltage adjusted until $\hat{\beta}_{y}(O N)=\hat{\beta}_{y}(O F F) . \hat{F}_{z}$ differs from $m g$ by two systematic error terms. The dynamical error term involves the quantity $\tilde{\gamma}^{2}$ - and, as was discussed above, $\tilde{\gamma}$ should be less than $2.5^{\prime \prime}$ for the brass electrode at $V=9 \mathrm{kV}$ if $\mathrm{m}$ is to be determined to within $1 \mu \mathrm{g}$.

The relative static error for $\hat{F}_{z}$ is $\left(2 \hat{F}_{y}^{0} / \mathrm{mg}\right) \hat{\beta}_{y}(\mathrm{OFF}) \cdot \hat{F}_{y}^{0}$ is predetermined by the relative electrode alignments and displacements (i.e. by $\hat{h}_{y}$ ) and by $v^{2}$. Therefore, this error term must be minimized by adjusting $\hat{\beta}_{y}{ }^{(O F F)}$ to be near zero. If the brass electrode is used at $V=9 \mathrm{kV}$, and if $d_{x}=d_{y}=\theta_{x}=0$ and $\theta_{y}=5 "$, then the plane defined by the balance beam knife edges must be determined to be parallel to within \pm 7 " of gravitational horizon in order for the static error term of $\hat{F}_{z}$ to be less than $1 \mathrm{ppm}$ - and, if the counterweight adjustment for obtaining this $\hat{\beta}_{y}(O F F)$ reading is incorrect by $64 \mu \mathrm{g}$, then an additional maximum $1 \mathrm{ppm}$ error could occur. In order to 
achieve a $1 \mu \mathrm{g}$ in $5 \mathrm{gram}$ precision for this example, the knife edges plane must be determined to within $\pm 1^{\prime \prime}$, and $M_{B}$ (or $M_{A}$ ) must be adjusted to within $10 \mu \mathrm{g}$ to obtain the balance beam readout angle value that corresponds to gravitational horizon. This adjustment could be made electronically if the balance beam was servoed.

This $\pm 1 \mu \mathrm{g}$ in $5 \mathrm{gram}$ precision would be very difficult to achieve with the brass suspended electrode at $V=9 \mathrm{kV}$. If an aluminum electrode was used at $V=4 \mathrm{kV}$ and $\theta_{y}=5^{\prime \prime}$, then Table 3 provides the information that the knife edges plane must be determined to within 16 " of gravitational horizon in order to determine $\hat{F}_{z}$ to within $1 \mathrm{ppm}$. If $M_{B}$ was out of adjustment by $150 \mu \mathrm{g}$ then another $1 \mathrm{ppm}$ error could occur. An additional $1 \mathrm{ppm}$ dynamic systematic error arises if $\tilde{\gamma}=6^{\prime \prime}$ for this case. Results for a brass electrode at $V=4 \mathrm{kV}$ and $\theta_{y}=5^{\prime \prime}$ are only marginally better than these aluminum electrode requirements.

Because of these systematic error terms, these sample calculations suggest that little, or nothing, may be gained in the force determinatio measurements by increasing the masses $M_{A}$ and $M_{B}$. The brass electrode would, however, provide a better safety margin against unstable equilibrium, and it also would enable data to be obtained at voltages larger than $4 \mathrm{kV}$.

The exact requirements for the readout angle determination and the $M_{B}$ or $M_{A}$ adjustment depend upon the largest possible combination of $d_{x}, d_{y}, \theta_{x}$ and $\theta_{y}$ that occurs during a $\Delta z_{L}$ scan. These $\theta_{y}=5^{\prime \prime}$ examples may, or may not, give an upper limit for the $\hat{\beta}_{y}$ (OFF) requirements: $\theta_{x}$ and $\theta_{y}$ may each be about $3^{\prime \prime}$, and $d_{x}$ and $d_{y}$ are unknown. Also, all the electrodes are assumed to have radial symmetry, and to have been perfectly aligned at some point $z_{L} ; \underline{i}$.e. 
the capacitance value at that point was at the absolute minimum and not at a local minimum. The horizontal forces would thus be zero at that point. If this were not the case, then a $\Delta \theta_{y}=+3 "$ variation, for example, would produce $\theta_{y}$ values larger or smaller than $3^{\prime \prime}$, depending upon the initial value of $\theta_{y}$.

The systematic error terms of $\hat{F}_{z}=m g$ do not occur for normal mass intercomparisons because $\mathrm{mg}$ is constant for a swinging balance pan and there are no horizontal forces. In this experiment, however, $\hat{F}_{z}$ is not constant and horizontal forces do occur in the voltage-on measurements.

3.4 Verification of the equations of motion

The solutions to the equations of motion are verified using Newton's second law of motion in Appendix R by investigating the time-dependence of the vertical components of the external forces induced on the moving suspended electrode as shown in Figure 12 . The shapes and frequency dependence of these forces ( $p$ lotted in Figures 13-17 versus time for a brass electrode at $V=0 \mathrm{kV}$ and at $V=9 \mathrm{kV}$ ) agree with the predictions of Appendix $P$, but the predictions from Equations $P-21$ and $P-22$ for the magnitudes of the $\Delta m$ mass variations, as measured by the balance, do not agree with those of Figure 18 because the balance acts like a low-pass frequency filter since $\omega_{\gamma_{y}} \gg \omega_{\beta_{y}}$. This frequency filtering explains why the amplitude modulations of the simple harmonic $\beta_{y}$ waveform are so small. Therefore, the only significant problem with the pendulum being in motion during the force 
measurements is with the dynamical error term of $\hat{F}_{z}$ due to the $\tilde{r}^{2}$ quantity affecting the value of $\hat{\beta}_{y}(O N)$.

\subsection{Dynamical capacitance measurements}

The suspended electrode executes simple harmonic motion about the equilibrium angle $\hat{\gamma}$. At the $V_{r m s}=100 \mathrm{~V}$ voltage value for the capacitance measurements, $\hat{\gamma}_{x}$ and $\hat{\gamma}_{y}$ are essentially zero.

The time variation of capacitance is given by Equation $S-2$ of Appendix $S$, and from that expression the quantity $\delta C(t) / C\left(z_{L}\right)=[C(t)$ - $\left.C\left(z_{L}\right)\right] / C\left(z_{L}\right)$ is obtained in Equation $S-3$. This quantity is plotted versus time in Figure 19 for a brass suspended electrode at $C\left(z_{L}\right)=$ $100 \mathrm{pF}$ and $d_{x}=d_{y}=\theta_{x}=0$, assuming various values of $\tilde{\gamma}_{y}$ and $\theta_{y}$. The only difference for an aluminum electrode would be that the pendulum period decreases from $1.5 \mathrm{~s}$ to $1.3 \mathrm{~s}$.

Perfect electrode alignment produces a pure $\cos 2 \omega_{\gamma_{y}} t$ signal. The $\cos \omega_{y} t$ component becomes more enhanced with increasing electrode misalignments and relative displacements. Therefore, the capacitance will increase symmetrically about $\hat{\gamma}=0$ only for perfect electrode alignments. So, dynamical capacitance measurements could be used for a deliberately swinging suspended electrode to find the absolute minimum in capacitance at some point $z_{L}$ by maximizing the $\cos 2 \omega_{\gamma_{y}} t$ signal via adjustments of $d_{x}, d_{y}, \theta_{x}$ and $\theta_{y^{*}}$

The pendulum amplitude must be quite small when making actual capacitance measurements - and, the larger the electrode misalignments, the smaller this ampititude must be. In general, $\tilde{\gamma}$ probably needs to be less than $5^{\prime \prime}$ in order to measure the static 
capacitance value to within $1 \mathrm{ppm}$ if the bridge detector averages $C$, or less than 10" if the minimum value of $C$ is used.

The dynamic capacitance values could only be safely used if the pendulum amplitudes were similar to those used in the force determination measurements; e.g. a $2.6 \mathrm{ppm}$ error would occur if $\theta_{y}=5^{\prime \prime}$, and if $\tilde{\gamma}=10^{\prime \prime}$ in one type of measurement and $\tilde{\gamma}=0$ in the other type.

We found in Appendix $C$ that the balance beam must be either mechanically braked or electronically servoed so that the electrode overlap does not change by more than $\pm 0.041 \mu \mathrm{m}$ in order not to change the capacitance to within $\pm 1 \mathrm{ppm}$ at $\mathrm{C}=50 \mathrm{pF}$. If a mechanical brake is used with its axis parallel to the center knife axis, and if it should rotate the balance beam about the $z$ axis, then this rotation will produce a suspended electrode displacement, $D_{x}$, of $\pm 0.36 \mu \mathrm{m}$ for each arcsecond of rotation, with the sign depending upon the direction of rotation.

This effect, if present, could be monitored by mounting the balance readout angle mirror in the $x-z$ plane rather than the $x-y$ plane; any balance beam rotation would thus cause a parallel shift in the light beam at the readout detector position. This mirror arrangement could also detect any balance beam rotation that might result from the balance being placed in arrestment [4] and then released.

If the upper part of the laser interferometer is not connected to the suspended electrode system, and if the brake stopped the balance beam to within \pm 0.11 " of the $\hat{\beta}_{y}{ }^{(O F F)}$ readout angle for the capacitance measurements, then there would be a $\pm 0.041 \mu \mathrm{m}$ uncertainty in the $\mathrm{z}_{\mathrm{L}}$ 
position of this capacitance reading, in addition to the $\pm 0.025 \mu \mathrm{m}$ laser interferometer reproducibility. In a $\Delta z_{L}=2.5 \mathrm{~cm}$ scan between capacitance measurement endpoints, this could give a maximum error of $\pm 5 \mathrm{ppm}$ in $\Delta z_{\mathrm{L}}$. The error could be as large as $\pm 3 \mathrm{ppm}$ for $\Delta z_{L}=4 \mathrm{~cm}$. The most probable errors for these two examples would be $\pm 3 \mathrm{ppm}$ and \pm 2 ppm respectively.

The same errors would also occur for a balance beam servoed to within \pm 0.11 " of the $\hat{\beta}_{y}(\mathrm{OFF})$ readout angle - providing the zero-point level of the servo was known be stable enough to not cause additional shifts on the order of 0.11 " during the course of the measurements. Therefore, it would be preferable to mount the upper part of the laser interferometer on the suspended electrode system.

It does not matter if the capacitance values are measured at exactly the same $z_{L}$ points as those used in the force determination measurements - as long as the force is nearly constant, or is slowly varying over reasonable changes in $z_{L}$. Therefore, a capacitance measurement could be made at any braked position, and the $z_{L}$ value correctly measured if the laser interferometer has the latter configuration. The capacitance could also be measured while using a servoed balance and this interferometer configuration, with the possible $\pm 0.041 \mu \mathrm{m}$ variations in the $z_{L}$ readings being monitored by the interferometer; $z_{L}$ could thus be determined to within $\pm 0.025 \mu \mathrm{m}$. Any zero-point drifts of the servo would automatically be accounted for.

Similar $z_{L}$ variations could occur during the force determination measurements when using a servoed balance beam. If the balance beam was freely-swinging with a $\hat{\beta}_{y}=4$ " amplitude, then $z_{L}$ would vary by 
$\pm 1.5 \mu \mathrm{m}$ ( \pm 150 times the $0.01 \mu \mathrm{m}$ laser interferometer resolution) during the $45 \mathrm{~s}$ period. If the force varies slowly with $z_{L}$, then the assigned value of $z_{L}$ for each measurement is not very critical. Therefore, the balance might be allowed to swing freely during the force determination measurements provided that $\hat{\beta}_{y}$ is small enough, and provided that the electrodes are nearly perfect right circular cylinders (so that the capacitances and the forces are not too dependent upon the electrode shapes). 


\subsection{The slope method}

Using Equations 2-19 and E-5 - and converting to appropriate multiples and submultiples for $V, C$ and $z_{L}$ - yields the equation

$$
\hat{F}_{z}\left(\hat{h}, z_{L}\right)=-(1 / 2)\left(1 \times 10^{-4}\right) v^{2}\left[\partial \hat{C}\left(\hat{h}, z_{L}\right) / \partial z_{L}\right]_{\hat{h}}, \hat{h}_{y}
$$

$\left[F_{Z}\right.$ in $N$; $V$ in $k V ; C$ in $p F ; \quad z$ in $\mathrm{cm} ; h$ in $\left.\mu \mathrm{m}\right]$.

$\hat{F}_{z}$ is the equilibrium value for the vertical component of the electrical force induced on the suspended electrode at the equilibrium point $\left(\hat{h}, z_{L}\right)$ of the relative coordinate system. It is assumed to be equal to $\mathrm{mg}$ in the force determination measurement when the measured voltage, $v_{\text {meas }}$, is adjusted until $\hat{\beta}_{y}^{(O N)}=\hat{\beta}_{y}^{(O F F)}$, resulting (by using Equations 2-16 and 2-19) in the equation

$$
\hat{F}_{z}\left(\hat{h}, z_{L}\right)=\left(6.06 \times 10^{-4}\right) V_{\text {meas }}^{2}\left(\hat{h}, z_{L}\right)\left[1+\left(6.5 \times 10^{-9}\right) \hat{h}^{2}\right]=m g(4-2)
$$

$\left[F_{Z}, m g\right.$ in $N$; $V$ in $k V ; z_{L}$ in $\mathrm{cm} ; h$ in $\left.\mu \mathrm{m}\right]$.

$V_{\text {meas }}$ is obtained via a resistance voltage divider and a group of standard cells which have been calibrated in terms of the NBS as-maintained volt. The mass $m$ can be determined from mass 
intercomparisons, and the gravitational acceleration, $g$, has been accurately measured at the suspended electrode position.

The quantity $\partial \hat{C} / \partial z_{L}$ in Equation $4-1$ is the slope of $\hat{C}$ versus $z_{L}$ evaluated at point $\left(\hat{h}, z_{L}\right)$ of the relative coordinate system. The capacitance can be measured as a function of $z_{L}$ by translating the carriage to different positions and monitoring $z_{L}$ via the laser interferometer system. The slope at point $\left(\hat{h}, z_{L}\right)$ can then be determined from the derivative of an nth-order polynomial which provides the best least-squares fit to the $\hat{C}$ versus $z_{L}$ data. Ideally, this slope should be obtained from carriage scans that keep $\hat{h}_{x}$ and $\hat{h}_{y}$ constant while changing $z_{L}$, but experimentally this is probably not possible.

Al1 the quantities in Equation 4-1, except $V$, can therefore be measured in SI units. So the SI voltage, $V_{c a l c}$, can be calculated from this equation and then compared with the measured voltage, $V_{\text {meas }}$, which is expressed in terms of the NBS as-maintained value.

There are, however, errors associated with the measurements of $\hat{F}_{Z}, \hat{C}$ and $z_{L}$ - and these errors affect the voltage ratio $V_{c a l c} / V_{\text {meas }}$. Sample calculations are presented in Appendix $T$ to gain an impression of how much this ratio could be changed by those errors. These calculations are not intended to be an error analysis, but rather to indicate how large the deviations in the voltage ratio might be. Part a of Appendix T explains why this approach is used.

The individual errors used in these calculations are: $\pm 2 \mathrm{ppm}$ for the combined static and dynamic systematic error of $\hat{F}_{z}$ in the force determination measurement (as discussed in Section 3.3 and part $g$ of Appendix P); $\quad a \pm 0.025 \mu \mathrm{m}$ uncertainty in the measurement of $z_{L}$ (as 
discussed in Section 3.5); a +1 ppm dynamic systematic error in the measurement of $\hat{C}$ (as given in Section 3.5 and Appendix $S$ ); and sometimes a $\pm 1 \mathrm{ppm}$ error in $\hat{C}$ due to the electrode overlap not being correctly monitored by $z_{L}$ because of temperature fluctuations (as discussed in Appendix D).

Various combinations of these errors are used in the examples of Appendix $T$, but one combination is, rather arbitrarily, chosen to obtain a possible spread in the voltage ratio values. This combination is: a $\pm 2 \mathrm{ppm}$ systematic error for $\hat{F}_{z}, a \pm 2 \mathrm{ppm}$ random error for $\Delta z_{L}$, and $a+1$ ppm dynamic systematic error for $\hat{C}$. No errors due to temperature fluctuations are assumed. (Hence, the slope error is determined from thi capacitance endpoint measurements in the examples rather than from a linear fit to a plot of $\hat{C}$ versus $z_{L}$ in which the values of $\hat{C}$ deviate from the straight 1 ine because of temperature fluctuations and dynamical measurement errors. The values of $z_{L}$ are all uncertain by $\pm 0.025 \mu \mathrm{m}$, so the slope of the linear fit might thus have an uncertainty of \pm 2 or 3 ppm, but one would require real data before actually assigning an uncertainty.)

These errors are combined in such a way as to give the largest effects. This combination is perhaps pessimistic. However, oniy the three errors given in the above paragraph are involved in the combination and some of them are random, some are systematic and some are correlated; so the errors are just as likely to add as to cancel. Also, an ideal measurement situation is assumed with perfect right cylinders, no balance zero-point drifts, and no curvature in the $\hat{c}$ versus $z_{L}$ function. In addition, no experimental errors are included 
for $V_{\text {meas }}$ due to the voltage divider step-down or to the comparisons with the NBS volt via the group of standard cells, or to the $\pm 1 \mathrm{ppm}$ random uncertainty in $\mathrm{m}$ at $\mathrm{V}=4.02 \mathrm{kV}$.

Three different carriage paths are assumed for the calculations of Appendix T. They are shown in Figure 20. The carriage moves along the symmetry axis from initial values of $z_{L}=0 \mathrm{~cm}$ and $C\left(z_{L}\right)=100 \mathrm{pF}$ to final values of $z_{L}=2.5 \mathrm{~cm}$ and $C\left(z_{L}\right)=69.7 \mathrm{pF}$ in the Type I scan. In the Type II scan the carriage moves such that $\hat{h}_{y}$ is constant at low voltages (i.e. for the capacitance measurements). (Note from Tables 9 and 10 that $\hat{h}_{y}$ is not constant at high voltages because the values of $\hat{\gamma}_{y}$ decrease with increasing $z_{L}$ values. Thus, the horizontal forces, and $\hat{F}_{z}$ also decrease.) Finally, $\hat{h}_{y}$ increases or decreases linearly with increasing $z_{L}$ at low voltages in the Type III scans. There are additional errors introduced in the Type II and III scans because $\hat{F}_{z}$ is measured at high voltages, while $\hat{C}$ is measured at low voltages where the suspended electrode remains nearly vertical - and also the slope is not determined at constant $h$.

Examples $4,8,17-20$, and $25-28$ of Appendix $T$ imply that the measured ratio of the SI volt and the NBS volt would be uncertain by +3.5 ppm and $-2.5 \mathrm{ppm}$ for a brass suspended electrode at $V=9 \mathrm{kV}$, resulting in a $3 \mathrm{ppm}$ measurement. Additional data would not reduce that part of the uncertainty due to systematic errors - such as the $\hat{F}_{z}$ measurements; also, the nearly repeatable carriage movements would always apply the same bias to the results, but this bias cannot be corrected unless $\gamma_{x}, \gamma_{y}, \theta_{x}, \theta_{y}$ and $d_{x}$ and $d_{y}$ are known at each $z_{L}$ point, so that $h$ could therefore be determined. 
Examples 10-11, 21-24, and 29-32 of Appendix T imply that the measured ratio of the SI volt and the NBS volt would be uncertain by +2 ppm and $-2.5 \mathrm{ppm}$ for an aluminum suspended electrode at $V=4.02 \mathrm{kV}$, resulting in a 2 or $3 \mathrm{ppm}$ measurement. (Note that $V_{\text {NBS }}$ is assumed to be equal to $V_{S I}$ in the examples. It does not matter if the experimental voltage ratio is not actually equal to one - the above uncertainty still applies.)

Another approach to estimating the uncertainty in the voltage ratio is to assume a $\pm 2 \mathrm{ppm}$ error in $\hat{\mathrm{F}}_{z}$ and a \pm 2 or $3 \mathrm{ppm}$ error in the slope $\partial \hat{C} / \partial z_{L}$, and to combine the errors by the square root of the sum of their squares. This yields an uncertainty of \pm 1.5 or $2 \mathrm{ppm}$ for both the brass and aluminum electrodes. Or, if the two errors are combined by addition, the uncertainty in the voltage ratio is \pm 2 or $3 \mathrm{ppm}$. This uncertainty does not include errors due to $\hat{F}_{z}$ being measured at high voltages while $\hat{C}$ is measured at low voltage, or the uncertainty in $m$.

It would appear to be difficult to obtain a value of the voltage ratio with an uncertainty less than \pm 2 or $3 \mathrm{ppm}$ by the slope method under ideal conditions. The uncertainty could be much larger in reality because of the very strong electrode shape-dependence of $\hat{C}$ (as discussed in part $f$ of Appendix $C$ ). The plot of $\hat{C}$ versus $z_{L}$ could have a large curvature - with a shape that might change along the carriage path - and would thus greatly increase the uncertainty in the derivative of the best lease-squares fit of the nth order polynomial to the data. Also, the laser interferometer uncertainty in the position of $z_{L}$ at which $\hat{F}_{Z}$ is measured becomes more important if the slope of $\hat{C}$ versus $z_{L}$ is rapidly changing. 


\subsection{The path integral method}

Appendix $U$ derives the path integral for the mechanical work

required to move the electrodes from an initial point $\left(\hat{h}_{x_{j}}, \hat{h}_{y_{j}}, z_{L_{i}}\right)$ in the relative coordinate system to a point $\left(\hat{h}_{x_{j}}, \hat{h}_{y_{j}}, z_{L_{j}}\right)$. The expression for the path integral is greatly simplified if the measured force $\hat{F}\left(\hat{h}, z_{L}\right)$ at each point is adjusted to a new value $\hat{F}^{\prime}\left(\hat{h}, z_{L}\right)$, where $\hat{F}^{\prime}$ is the value that $\hat{F}$ would have had if $V_{\text {meas }}\left(\hat{h}, z_{L}\right)$ were constant at every point of the carriage scan. The adjustment factor, $\xi\left(\hat{h}, z_{L}\right)=$ $\nabla^{2} / v_{\text {meas }}^{2}\left(\hat{h}, z_{L}\right)$, for $\hat{F}\left(\hat{h}, z_{L}\right)$ is obtained in Appendix $v ; \quad \bar{V}$ is either the average of the $V_{\text {meas }}\left(\hat{h}, z_{L}\right)$ values, or any other arbitrary normalization value - such as the nominal voltage $V_{N}$.

The combination of Equations U-5, V-4, 2-14, 2-15 and 2-16 gives

$$
\begin{aligned}
w^{e}= & \left.\left(6.5 \times 10^{-13}\right) \int_{h_{x i}}^{h_{x}} \hat{h}_{j}, z_{L}\right) v_{\text {meas }}^{2}\left(\hat{h}, z_{L}\right) c\left(z_{L}\right) \hat{h}_{x} d h_{x} \\
& +\left(6.5 \times 10^{-13}\right) \int_{y_{j} \xi\left(\hat{h}, z_{L}\right) v_{\text {meas }}^{2}\left(\hat{h}, z_{L}\right) C\left(z_{L}\right) \hat{h}_{y} d h_{y}}^{h_{y j}} \\
& -\left(6.06 \times 10^{-4}\right) \int_{L_{j} \xi\left(\hat{h}, z_{L}\right) v_{\text {meas }}^{2}\left(\hat{h}, z_{L}\right) \cdot}^{z_{L}} \\
& \left\{1+\left(6.5 \times 10^{-9}\right)\left(\hat{h}_{x}^{2}+\hat{h}_{y}^{2}\right)\right\} d z_{L} \\
= & \left(1 \times 10^{-4}\right)(1 / 2) \bar{v}^{2}\left[\hat{C}_{j}\left(\hat{h}, z_{L}\right)-\hat{C}_{j}\left(\hat{h}, z_{L}\right)\right]
\end{aligned}
$$

[W in $\mathrm{N} \cdot \mathrm{cm} ; \quad V$ in $\mathrm{kV} ; \quad \mathrm{C}$ in $\mathrm{pF} ; \mathrm{z}_{\mathrm{L}}$ in $\mathrm{cm} ; \mathrm{h}$ in $\left.\mu \mathrm{m}\right]$.

Note that since $h_{x}, h_{y}, F_{h_{x}}$ and $F_{h_{y}}$ contain information about $\theta_{x}, \theta_{y}$, $\gamma_{x}$ and $\gamma_{y}$, that the mechanical work integral $w^{e}$ includes not only the translational work $-\int \vec{F} \cdot d \vec{l}$ due to 1 inear motion of the carriage electrodes, but al so the rotational work integrals $-\int \vec{\Gamma}_{\gamma} \cdot d \vec{\gamma}$ and $-\int \vec{\Gamma}_{\theta} \cdot d \vec{\theta}$ 
due to changes in tilts of the suspended and carriage electrodes, respectively.

The path integral represented by Equation 4-3 must be conservative. Appendix $W$ verifies that this is indeed the case. Any path may be used - with any normalization voltage $\bar{V}$ - but, in order to compare several paths, the value of $\bar{V}$ must be the same for each path. So, the normalization voltage might just as well be the nominal value, $V_{N}$ of the voltage applied to the electrodes, i.e. $V_{N}=9 \mathrm{kV}$ for $m=5$ grams and $V_{N}=4.02 \mathrm{kV}$ for $m=1$ gram.

Equation 4-3 was mathematically useful to show in Appendix $W$ that the path integral is conservative, but, experimentally, the more convenient combination of Equations $U-5$ and $V-4$ is

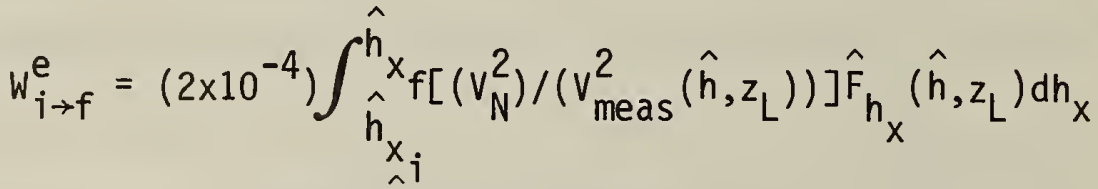

$$
\begin{aligned}
& +\left(2 \times 10^{-4}\right) \int_{\hat{h}_{y_{i}}}^{\hat{h}_{y_{f}}}\left[\left(v_{N}^{2}\right) /\left(v_{\text {meas }}^{2}\left(\hat{h}, z_{L}\right)\right)\right] \hat{F}_{h_{y}}\left(\hat{h}, z_{L}\right) d h_{y} \\
& -\int_{z_{L_{j}}}^{z_{L_{f}}}\left[\left(v_{N}^{2}\right) /\left(v_{\text {meas }}^{2}\left(\hat{h}, z_{L}\right)\right)\right]\left|\hat{F}_{z_{L}}\left(\hat{h}, z_{L}\right)\right| d z_{L} \\
& =\left(1 \times 10^{-4}\right)(1 / 2) v_{c a l c}^{2}\left[\hat{C}_{f}\left(\hat{h}_{f}, z_{L_{f}}\right)-\hat{C}_{j}\left(\hat{h}_{j}, z_{L_{j}}\right)\right]
\end{aligned}
$$

$\left[W^{e}\right.$ in $N \cdot c m ; V_{N}, V_{\text {meas }}, V_{\text {calc }}$ in $\mathrm{kV} ; F_{h}, F_{Z}$ in $N ; C$ in $p F ; h$ in $\mu \mathrm{m}$; $z_{L}$ in $\left.\mathrm{cm}\right]$.

Some problems in evaluating this equation are immediately apparent: $\hat{F}_{\hat{h}_{x}}$ and $\hat{F}_{\hat{h}_{y}}$ cannot be measured with the balance, and $\hat{h}_{x}$ and $\hat{h}_{y}$ are also 
unknown at each point $\left(\hat{h}_{x}, \hat{h}_{y}, z_{L}\right)$ of the relative coordinate system because only $\theta_{x}, \theta_{y}, \gamma_{x}$ and $\gamma_{y}$ could be conveniently monitored via autocollinators. The measurements of $d_{x}$ and $d_{y}$ are difficult and involve expensive laser interferometry techniques. Therefore, the horizontal parts of the path integrals are unknown. Calculations of Appendix $X$ show that the missing horizontal integral contributions to the path integral can be quite large, with the amounts depending upon the particular path taken. The horizontal integral contributions are 11 ppm and $15 \mathrm{ppm}$ for the two paths used in that appendix.

Another problem arises from $\hat{F}_{z}$ being measured at high voltages where the suspended electrode has, in general, swung out to some equilibrium angle $\hat{\gamma}$ - whereas, $\hat{C}$ is measured at low voltages where the suspended electrode is essentially vertical. So, the capacitance measurement is not made with the same electrode configuration as that for the force measurement.

Appendix $Y$ provides sample calculations of Types I, II and III scans for a brass suspended electrode at $V_{N}=9 \mathrm{kV}$ and for an aluminum electrode at $V_{N}=4.02 \mathrm{kV}$. The errors chosen to obtain a possible spread in the voltage ratio values $V_{c a l c} / V_{N}$ are: a $\pm 2 p p m$ systematic error for $\hat{F}_{z}$ and none for $m, a \pm 2 \mathrm{ppm}$ random error for $\Delta z_{L}$, and $a+1 \mathrm{ppm}$ dynamic systematic error or temperature fluctuation effect error for one of the capacitance endpoint measurements. (More emphasis must be given to the possible capacitance measurement errors in the path integral method because this method uses the capacitance endpoint values, and therefore one cannot average the capacitance deviations over the $\Delta z_{L}$ scan as in the slope method.) (The largest canacitance errors might be 
$+2 \mathrm{ppm}$ at one endpoint and $-1 \mathrm{ppm}$ at the other, but this combination is very unlikely.)

The errors are combined additively in the examples of Appendix $Y$ because some of them are random, some are systematic and some are correlated - and because perfect right cylindrical electrodes are assumed, along with no balance zero-point drifts. The ratio $V_{c a l c} / V_{N}$ is evaluated with the experimentally unknown horizontal parts of the path integral not included, and with the capacitance values not being corrected to the values that they would have had at high voltages; i.e. only measured quantities, and their assumed experimental uncertainties, are used in the voltage ratio calculations. Part a of Appendix $Y$ explains the reasons for using this approach.

Examples 2-3, 8-9, and 13-14 of Appendix $Y$ imply that the measured ratio of the SI volt and the NBS volt would be uncertain by $+4.5 \mathrm{ppm}$ and $-3.5 \mathrm{ppm}$ for a brass suspended electrode at $V_{N}=9 \mathrm{kV}$, resulting in a 4 ppm measurement. Additional data would not reduce that part of the uncertainty due to systematic errors. Also, the nearly repeatable carriage movements would always apply the same bias to the results, but this bias cannot be corrected unless $\gamma_{x}, \gamma_{y}, \theta_{x}, \theta_{y}$ and $d_{x}$ and $d_{y}$ are known at each point $z_{L}$.

Examples $3,6,11-12$, and 15-16 of Appendix $Y$ imply that the measured ratio of the SI volt and the NBS volt would be uncertain by $+3.5 \mathrm{ppm}$ and $-4 \mathrm{ppm}$ for an aluminum suspended electrode at $V_{\mathrm{N}}=4.02 \mathrm{kV}$, resulting in a 3 or $4 \mathrm{ppm}$ measurement. 
Another approach for estimating the uncertainty in the voltage ratio is to assume $\pm 1 \mathrm{ppm}$ systematic static and dynamic errors in $\hat{F}_{z}$, a $\pm 1 \mathrm{ppm}$ random error in $\mathrm{m}, \mathrm{a} \pm 1.4 \mathrm{ppm}$ random error in $\Delta z_{L}$, and a $\pm 1.4 \mathrm{ppm}$ error in $\left(\hat{C}_{f}-\hat{C}_{j}\right)$ - and to then combine these errors by taking the square root of the sum of the squares of the individual errors for each type of scan given in Appendix $Y$. The ratio would be uncertain in this approach by $+2.5 \mathrm{ppm}$ and $-1.5 \mathrm{ppm}$ for both a brass electrode at $V_{N}=9 \mathrm{kV}$ and for an aluminum electrode at $V_{N}=4.02 \mathrm{kV}$ - resulting in a $2 \mathrm{ppm}$ measurement. This uncertainty is, however, probably optimistic. A more conservative estimate might be that 3 or $4 \mathrm{ppm}$ measurements could be obtained with either a brass electrode at $V_{N}=9 \mathrm{kV}$ or an aluminum electrode at $V_{N}=4.02 \mathrm{kV}$.

Unlike the slope method, it is not essential that $\hat{C}$ versus $z_{L}$ be nearly a straight line with no changes in its shape, (or therefore that $\hat{F}_{Z}^{\prime}$ versus $z_{L}$ be nearly constant). If $\hat{F}_{Z}^{\prime}$ changes dramatically, then $\hat{F}_{z}$ must be measured at more points so that the area under the curve of $\hat{F}_{Z}^{\prime}$ versus $Z_{L}$ (analogous to that of Figure 22 for perfect right cylinders) can be obtained with sufficient accuracy. 
5.1 Problems in measuring $\hat{\mathrm{F}}_{\mathrm{z}}$

This section summarizes the results of the calculations presented in Sections 2 through 4 and Appendices $A$ to $Y$, and also states the requirements or conditions that probably must be satisfied in order to reduce the systematic and random errors of the measurements to acceptable levels so that the SI volt can be determined to within a few ppm.

If the four electrode surfaces are not perfectly aligned then large horizontal forces occur on the suspended electrode at high voltages because most of the electric field lines are radially directed. These horizontal forces may typically be on the order of a few percent of the vertical electrical force value $\hat{F}_{z}$.

The relative alignments of the three cylinders are critical in reducing the size of the horizontal forces. They must be properly oriented at some point $z_{L}$ and then maintained over a $\Delta z_{L}$ span of about $2.5 \mathrm{~cm}$ such that the relative electrode tilts and displacements deviate by no more than a few seconds of arc and a few micrometers from perfect alignment at each $z_{L}$ value for both the $x$ and $y$ components of $d, \theta$ and $\gamma$.

Note that the calculations assumed that the carriage electrodes were perfectly aligned with respect to each other so that $h^{I}=h^{0}$, $c^{I}=c^{0}$ and $F_{h}^{I}=F_{h}^{0}$. The same alignment requirements of a few 
seconds of arc and a few micrometers also occur if $h^{I} \neq h^{0}$. The deviation of any of the four electrode surfaces from the combined symmetry axis results in horizontal forces.

The carriage platform presently changes its tilt by several seconds of arc in both the $\theta_{x}$ and $\theta_{y}$ directions during $\Delta z_{L}$ scans. The $d_{x}$ and $d_{y}$ changes are unknown. It is also presently unknown just how well the four electrode surfaces can be mutually positioned and oriented with respect to the combined electrodes electrical symmetry axis. Many of the sample calculations have used $d_{x}=d_{y}=$ $0, \gamma_{x}=\gamma_{y}=0$ for voltage-off, and $\theta_{x}=5^{\prime \prime}$ or $\theta_{y}=5^{\prime \prime}$ to provide $h_{x}$ or $h_{y}$ values that might experimentally occur at some points of the carriage scans.

A brass suspended electrode remains in stable equilibrium at $V=9 \mathrm{kV}(\mathrm{m}=5 \mathrm{grams})$; whereas an aluminum suspended electrode is sufficiently stable at $V=4.02 \mathrm{kV}(\mathrm{m}=1 \mathrm{gram})$, but its equilibrium angle becomes too 1 arge at $V=5.68 \mathrm{kV}(\mathrm{m}=2$ grams $)$.

In general, the suspended electrode executes simple harmonic motion about an equilibrium angle $\hat{\gamma}$. This motion can be that of an elliptical pendulum, a conical pendulum, a simple pendulum whose swept-out plane can be perpendicular to any direction in the $x-y$ plane, or it can be at rest at the angle $\hat{\gamma}$.

The pendulum period is voltage-dependent, and for a brass suspended electrode increases from a value of $1.5 \mathrm{~s}$ at $V=0 \mathrm{kV}$ to a value of $2.8 \mathrm{~s}$ at $V=9 \mathrm{kV}$. The al uminum electrode period increases from a value of $1.3 \mathrm{~s}$ at $V=0 \mathrm{kV}$ to $2.0 \mathrm{~s}$ at $4.02 \mathrm{kV}$. 
The balance also executes simple harmonic motion about an equilibrium angle $\hat{\beta}_{y}$. The period of this motion is voltage-dependent, and for a balance sensitivity of $0.11 " / \mu \mathrm{g}$ increases from a value of $44.9 \mathrm{~s}$ at $V=0 \mathrm{kV}$ to $46.8 \mathrm{~s}$ at $\mathrm{V}=9 \mathrm{kV}$ with a brass suspended electrode. Since the pendulum and the balance beam periods are so different, only very small energy transfers occur between the $\gamma$ and $\beta$ motions. Therefore, the pendulum and balance beam amplitudes do not alternately, and periodically, increase and decrease with time.

The balance acts as a low-pass frequency filter. Thus, the amplitude modulations (due to the suspended electrode motion) are undetectable on the simple harmonic $\beta_{y}$ waveform for small pendulum amplitudes since $\omega_{\gamma} \gg \omega_{\beta}$. (Note that if the pendulum amplitude $\tilde{\gamma}$ is greater than 7" then the $\beta_{y}$ waveform becomes more like a Mathieu function rather than a simple sine or cosine function.)

A systematic error does occur in the force determination measurements, however, because the balance beam equilibrium angle depends upon $\tilde{\gamma}^{2}$ at high voltages. A 1 ppm dynamical error in the $\hat{F}_{z}$ value occurs in both a brass suspended electrode at $V=9 \mathrm{kV}$ and an aluminum electrode at $V=4.02 \mathrm{kV}$ if $\tilde{\gamma}=6 "$. If this dynamical error in $\hat{F}_{z}$ were to be less than $0.2 \mathrm{ppm}$ (so that advantage were taken of the $1 \mu \mathrm{g}$ resolution in 5 grams at $V=9 \mathrm{kV}$ ) then $\tilde{\gamma}$ must be less than 2.5". 
The sign of the error is such that $\hat{F}_{z}$ always appears larger than mg for a swinging suspension system. This geometry-dependent systematic error would still occur at high voltages for perfect electrode alignment, but it does not occur for normal mass intercomparisons.

No damping terms were included in the calculations, but the pendulum amplitude would decay exponentially if there are no air currents. It is not presently known what the time constant would be. It could be quite long because of the small friction of the load knives and the small velocities for the viscous damping forces, but only experience will tell.

Another systematic error can also occur in the force determination measurements if the $y$ component of the horizontal electrical force on the suspended electrode is nonzero $\left(\hat{F}_{y}^{I}+\hat{F}_{y}^{0} \neq 0\right)$ and if the equilibrium angle of the plane defined by the contact points of the balance beam center knife and the load knife is not parallel with gravitational horizon $\left(\hat{\beta}_{y}^{(O F F)} \neq 0\right)$. A torque, $\left(\hat{F}_{y}^{I}+\hat{F}_{y}^{0}\right) L_{A} \sin \hat{\beta}_{y}$, occurs about the center knife in this case, and - since this torque is linear in $\hat{\beta}_{y}$ for small $\hat{\beta}_{y}$ - there is no minimum for $\hat{F}_{z}$ versus $\hat{\beta}_{y}$. This problem does not occur in normal mass intercomparisons because there are no applied horizontal forces.

If the carriage platform is displaced or changes its tilt during carriage scans then horizontal forces are present on the suspended electrode. The only way to reduce this static error term (without adjusting $d_{x}, d_{y}, \theta_{x}$ and $\theta_{y}$ in order to minimize the horizontal force 
at each position of the carriage scan) is to assure that $\hat{\beta}_{y}^{(0 F F)}$ is nearly zero.

If a brass suspended electrode is used at $V=9 \mathrm{kV}$, and if $d_{x}=d_{y}=\theta_{x}=0$ and $\theta_{y}=5 "$, then a systematic \pm 1 ppm error would occur in the $\hat{F}_{z}$ measurement at $\hat{C}=100 \mathrm{pF}$ if the knife edges plane (i.e. $\hat{\beta}_{y}^{(O F F)}$ ) was \pm 7 " from gravitational horizon. The same error would occur for an aluminum electrode at $V=4.02 \mathrm{kV}$ if $\hat{\beta}_{y}^{(O F F)}= \pm 16 "$. The plane of the knife edges would have to be determined to within \pm 1 " of gravitational horizon in order to obtain a $\pm 0.2 \mathrm{ppm}$ error in $\hat{F}_{z}$ for the brass electrode at $V=9 \mathrm{kV}$. This does not seem possible.

$A \hat{\beta}_{y}^{(O F F)}= \pm 16^{\prime \prime}$ requirement corresponds to determining that the vertical height of the contact point $(P)$ of the load knife in Figure 2 is within $\pm 6 \mu \mathrm{m}$ of the height of the contact point (C) of the center knife. (Six micrometers is about ten wavelengths of red light.) The $\hat{\beta}_{y}^{(O F F)} \approx 0$ determination would surely need to be done with the balance under load in order to account for knife edge and balance beam deformations. This determination would provide a balance beam readout angle that corresponds to $\hat{\beta}_{y}^{(O F F)} \approx 0$. The mirrors for the readout optics should not tilt after this readout angle determination, or a systematic error in $\hat{\beta}_{y}^{(\text {OFF })}$ would result.

The suspension system mass $M_{A}+m$ and the counterweight mass $M_{B}$ must be adjusted until the net torque about the center knife is close enough to zero, with the voltage turned off, that $\hat{\beta}_{y}^{(O F F)} \approx 0$; i.e. the desired readout angle value is obtained. This corresponds to another $\pm 1 \mathrm{ppm}$ systematic error in $\hat{F}_{Z}$ if $M_{A}+m$ differs from $M_{B}$ by 
about $\pm 65 \mu \mathrm{g}$ for a brass suspended electrode at $V=9 \mathrm{kV}$, or by about $\pm 150 \mu \mathrm{g}$ for an aluminum electrode at $V=4.02 \mathrm{kV}$ if $L_{A}=L_{B}$ and the mass distribution of the balance beam is symmetrical about the center knife.

If the balance beam was servoed then the balance could be trivially adjusted to the desired readout angle if the zero-point level of the feedback circuit was sufficiently stable. If the balance was not servoed, then either $M_{A}+m$ or $M_{B}$ would have to be adjusted to obtain the desired readout angle for a freely-swinging balance. The systematic mass adjustment error in $\hat{F}_{z}$ could either add to or subtract from the error associated with the knife edges plane determination.

The above discussion of possible static and dynamic systematic errors in $\hat{F}_{z}$ suggests that the uncertainties in the $\hat{F}_{z}$ determination would not be reduced by using higher voltages because the $\tilde{\gamma}$ and $\hat{\beta}_{y}^{(0 F F)} \approx 0$ conditions become too stringent. However, it is important to compare the SI volt with the NBS volt at different electrode voltages. Therefore, a brass suspended electrode, or an aluminum electrode with additional counterpoise mass placed along its symmetry axis near the lower rim is ultimately required because an al uminum electrode is not stable enough at voltages larger than $V=4.02 \mathrm{kV}$. The equilibrium angle $\tilde{\gamma}$ would also be smaller with more mass for a given horizontal force, thereby reducing the static error term of $\hat{F}_{z}$. 
Another possible systematic error in $\hat{F}_{z}$, which has not been mentioned, arises if net torques about the $x$ axis are induced on the suspended electrode. This could occur if the carriage electrodes pivoted about a point in the overlap region of the electrodes, so that the $y$ components of the inner and outer horizontal forces distributed along the suspended electrode formed a couple, and therefore a torque about the $x$ axis.

This $\hat{\Gamma}_{x}$ torque on the suspended electrode would appear as a force on the load knife at point (P), and it would essentially be in the $y$ direction. Thus, an additional torque would be induced about the balance beam center knife if the knife edges plane was again not at gravitational horizon. (Torques about the $y$ axis of the suspended electrode would try to twist the load knife and might thus change the length $L_{A}$ )

These torques on the suspended electrode could be detected if the connecting ring of Figure 1 was replaced with a gimbal device, and if the angles of both parts of the suspension system were monitored by autocollimators. This suspension system would act as a double pendulum, however, so the motion would be more complicated.

The $\hat{\Gamma}_{x}$ torque could be el iminated at only one point of the carriage scan. Therefore, this systematic error in $\hat{F}_{z}$ must be minimized by again assuring that $\hat{\beta}_{y}^{(O F F)} \approx 0$ as was the condition for reducing the static error term of $\hat{F}_{Z}$. 
$\hat{F}_{z}$ is assumed to be equal to mg when $\hat{\beta}_{y}^{(O N)}=\hat{\beta}_{y}^{(O F F)}$. However, the balance resolution and stability is $1 \mu \mathrm{g}$. Therefore, there is a $\pm 1 \mathrm{ppm}$ random uncertainty in the value of $\hat{F}_{z}$ at $V=4.02 \mathrm{kV}$ $(m=1 \mathrm{gram})$. This error decreases at higher voltages.

5.2 Problems in measuring $\hat{C}$

The equilibrium value of the capacitance $\hat{C}\left(\hat{h}, z_{L}\right)$ at any point $\left(\hat{h}_{x}, \hat{h}_{y}, z_{L}\right)$ is very sensitive to the value of $z_{L}$, where $z_{L}$ is a measure of the electrode overlap. The value of $\hat{C}$ changes by $\pm 1 \mathrm{ppm}$ at $C \approx 50 \mathrm{pF}$ if $\mathrm{z}_{\mathrm{L}}$ changes by $\pm 0.041 \mu \mathrm{m}$. (Note that $0.041 \mu \mathrm{m}$ is about one tenth a wavelength of blue light.)

The electrode overlap changes by $\pm 0.041 \mu \mathrm{m}$ if the balance beam angle changes by $\pm 0.11^{\prime \prime}$. So, the bal ance beam cannot be allowed to swing during the capacitance measurements. On the other hand, the suspended electrode system must remain free to swing; otherwise $\hat{C}$ would be measured for an electrode configuration that was different from that for $\hat{F}_{z}$.

There are two ways of not allowing the balance to swing: electronically servoing the balance beam to within a random stability of $\pm 0.11^{\prime \prime}$, or mechanically braking the balance beam along an axis parallel to, and above, the center knife axis. A balance beam servo could not be driven via the voltage applied to the electrodes because the beam must also be servoed for voltage-off measurements. A mechanical brake would have to virtually stop the balance beam from 
moving. If, in addition, it rotated the beam about the $x$ axis, the balance readout optics could be used to detect it.

If the mechanical brake rotates the balance beam about the $z$ axis by \pm 1 " then the suspended electrode would be displaced in the $x$ direction by $\pm 0.36 \mu \mathrm{m}$, thus changing the electrode configurations during the capacitance measurements. This effect could be checked by mounting the balance beam readout mirror in the $x-z$ plane rather than in the $x-y$ plane. This mirror arrangement would al so detect any balance beam rotations that might occur whenever the balance is placed in arrestment and then released.

When the voltage is off, the center of mass of the suspended electrode system (point 1 in Figure 2) will, by definition be directly below the pivot point $(P)$. The suspended electrode surfaces, however, will not necessarily be parallel with $g$ if there are any radial mass asymmetries. Therefore, there is a spider arrangement located at the top of the suspended electrode that allows the electrode surfaces to be oriented so they are nearly parallel to $\overrightarrow{\mathrm{g}}$.

The suspended electrode equilibrium angle $\hat{\gamma}$ will then be zero with the voltage turned off - and $\hat{\gamma}$ will remain essentially at zero at the $100 \mathrm{~V}$ a.c. used in the capacitance measurements because the horizontal forces are small. The capacitance measurements are thus only affected by changes in the displacements $d$ and tilts $\theta$ of the carriage electrodes if $\hat{\gamma}=0$. At the higher voltages used in the $\hat{F}_{z}$ measurements, however, $\hat{\gamma} \neq 0$ if there are horizontal forces on the 
suspended electrode. So, the actual capacitances are larger at high voltages than the measured capacitance. For example, if $d_{x}=d_{y}=\theta_{x}=0$ and $\theta_{y}=5 "$, then the measured capacitance equilibrium values are systematically too small by $4.4 \mathrm{ppm}$ for a brass suspended electrode at $V=9 \mathrm{kV}$, and by $0.6 \mathrm{ppm}$ for an aluminum electrode at $V=4.02 \mathrm{kV}$.

The balance beam must not be allowed to swing during the capacitance measurements, but the suspended electrode system should be free to swing so that it is not artifically displaced or tilted. If the suspended electrode is swinging during the capacitance measurements then $C$ will oscillate with time during the $1.5 \mathrm{~s}$ period of a brass electrode or the $1.3 \mathrm{~s}$ period of an aluminum electrode. (Some examples of these oscillations are shown in Figure 19.)

The capacitance bridge detector needs to have a short enough time constant so that it can correctly respond to these oscillations. If it does respond quickly enough, then the minimum reading, $C_{\min }$, is probably the best one to use for the static capacitance value $\hat{C}$. There might be a $\pm 1 / 2 \mathrm{ppm}$ random error in determining $C_{\min }$ if the pendulum amplitude $\tilde{\gamma}$ is $10^{\prime \prime}$, and a $-1 / 2 \mathrm{ppm}$ systematic error due to $\hat{\mathrm{C}}$ actually being larger than $C_{\min }$ if $\theta \approx 5 "$ and $d \approx 0$.

If the bridge detector averages the signal, then $\tilde{\gamma}$ probably should be less than $5^{\prime \prime}$ in order to measure $\hat{C}$ to within $\pm 1 \mathrm{ppm}$. The average value of the signal might be larger or smaller than the actual value of $\hat{C}$. 
The capacitance oscillation shapes could be utilised for aligning the electrodes. Perfect electrode alignment produces a pure $\cos 2 \omega_{\gamma} t$ signal. There is also a cos $\omega_{\gamma} t$ component at imperfect alignment and this component becomes more enhanced with increasing relative electrode misalignments and displacements. This effect could therefore be used to align the electrodes by delibrately swinging the pendulum and adjusting the displacements and tilts of the four electrode surfaces such that the $\cos 2 \omega_{\gamma} t$ component was maximized.

Friction in a gimbal might dampen the pendulum amplitude too quickly, and thus prevent this alignment technique from being used for a double pendulum configuration. Also, there would be two fundamental pendulum frequencies $\omega_{\gamma_{1}}$ and $\omega_{\gamma_{2}}$ and alternately increasing and decreasing pendulum amplitudes $\tilde{\gamma}_{1}$ and $\tilde{\gamma}_{2}$, so it might be too difficult to decipher the proper capacitance signal waveform for perfect electrode alignment when using a double pendulum. Another test of electrode alignment would be to observe the pendulum equilibrium angle $\hat{\gamma}$ at high voltages. If the electrodes are misaligned, then the horizontal forces would cause $\hat{\gamma}$ to be nonzero. This might not be as sensitive a check as the capacitance signal shape measurement because the carriage electrodes could be tilted in some direction and then displaced in the opposite direction such that the resulting horizontal forces (or torques about point $(P)$ ) were equal and opposite, such that $\hat{\gamma}=0$. The value of $\hat{C}$ would not be at its absolute minimum, however; so a $\cos \omega_{\gamma} t$ component would be present for a high enough capacitance bridge detector sensitivity. 
Part $f$ of Appendix $C$ demonstrates that the value of $\hat{C}$, at any point $\left(\hat{h}, z_{L}\right)$, is very sensitive to the overall shapes and the macroscopic bumps and valleys of the four electrode surfaces. If the average radius of the overlap region of any electrode surface changes by $\pm 1 \mu \mathrm{m}$ from the radius of a perfect right circular cylinder then the capacitance value changes by $\pm 54 \mathrm{ppm}$ from that of a right cylinder.

This is a real effect, not an error. However, it could introduce large curvatures and changes of shape in plots of $\hat{C}$ versus $z_{L}$, thereby producing greater uncertainties in the slope determination, $\partial \hat{C} / \partial z_{L}$, for the slope method and causing fluctuations in the values of $\hat{F}_{z}$ versus $z_{L}$.

If the temperature distribution within the 0 -ring sealed vessel changes by a few millidegrees Celsius while data are being obtained then the capacitance values assigned to each point $\left(\hat{h}, z_{L}\right)$ might vary by $\pm 1 \mathrm{ppm}$. These variations could be due to changes in the overall temperature or to changes in the vertical or the radial temperature gradients.

Fluctuations in the values of $\hat{C}$ versus $z_{L}$ would increase the uncertainty of the slope assignment, but they might be partially compensated with enough data. It would be more critical in the path integral method, however, because it is the capacitance values at the endpoints of the carriage scans that count. 


\subsection{Problems in measuring $z_{L}$}

The corner cube mirror of the laser interferometer is attached to the moveable carriage in Figure 1, while the upper part of the interferometer might, or might not, be attached to the suspended electrode system. The interferometer random uncertainty is \pm 0.025 $\mu \mathrm{m}$, and its resolution is $\pm 0.01 \mu \mathrm{m}$.

If the laser interferometer direction makes an angle of 90 " with respect to the combined electrodes symmetry axis then the resulting error in measuring changes of electrode overlap is only $0.1 \mathrm{ppm}$. It should not be difficult to attain this alignment condition.

If the balance beam is at rest and if the upper part of the interferometer is attached to the suspended electrode system, then the random uncertainty in $z_{L}$ is $\pm 0.025 \mu \mathrm{m}$. If the upper interferometer part is not attached, then an interferometer calibration of the change in electrode overlap for a change in balance beam readout angle must be made. This would allow a correction (with any resulting systematic errors in the calibration) to be made to $z_{L}$ if the balance beam was not mechanically braked at the readout angle corresponding to $\hat{\beta}_{y}^{(O F F)}=0$. (No correction need be applied to $z_{L}$ if the balance beam is at the desired readout angle.) In either event, another $\pm 0.041 \mu \mathrm{m}$ random uncertainty is combined with the $\pm 0.025 \mu \mathrm{m}$ interferometer uncertainty of $z_{L}$ if the readout optics uncertainty, or the balance servo uncertainty, is \pm 0.11 " and if the upper interferometer part is unattached. This would result for capacitance measurements in a maximum random error in $\Delta z_{L}$ of $\pm 5 \mathrm{ppm}$, 
or a probable error (using $\sqrt{2}$ times the square root of the sum of the squared uncertainties) of $\pm 3 \mathrm{ppm}$ for a $\Delta z_{L}=2.5 \mathrm{~cm}$ scan. If the upper interferometer part is attached then the maximum error in $\Delta z_{L}$ is $\pm 2 \mathrm{ppm}$ and the probable error is $\pm 1.4 \mathrm{ppm}$ for a $\Delta z_{L}=2.5 \mathrm{~cm}$ scan.

It is therefore preferable to attach the upper part of the interferometer to the suspended electrode system. However, its present mass is about 830 grams. So, it would be best for mechanical stability reasons to increase $L_{c m}$ by using this interferometer component counterpoise mass located on the suspended electrode symmetry axis near the lower rim, and to move the corner cube mirror to the bottom of the carriage platform. (A swinging electrode would cause parallel shifts in the return laser beam, but this intensity decrease is negligible for the small $\tilde{\gamma}$ amplitude restrictions in the capacitance and force measurements.)

If the upper part of the interferometer is not attached to the suspended electrode during the $\hat{F}_{z}$ measurements and if the voltage is adjusted until $\hat{\beta}_{y}^{(O N)}=\hat{\beta}_{y}^{(O F F)}$, then the maximum uncertainty in $z_{L}$ is $\pm 0.066 \mu \mathrm{m}$ and the more probable uncertainty is $\pm 0.048 \mu \mathrm{m}$, whether the balance beam is servoed or is freely-swinging. If the upper interferometer part is attached and the balance beam is servoed then the uncertainty in $z_{L}$ is $\pm 0.025 \mathrm{~cm}$. If it is attached, but the balance beam is freely-swinging with a $\tilde{\beta}_{y}=4$ " amplitude, then $z_{L}$ would vary by $\pm 1.5 \mu \mathrm{m}$ during the $45 \mathrm{~s}$ balance beam period. This might limit the accuracy in determining the value of $z_{L}$ corresponding to $\hat{F}_{z}$, but it is not a problem if $\hat{F}_{z}$ is constant or only a slowly 
varying function of $z_{L}$. If $\hat{F}_{z}$ varies rapidly with $z_{L}$ (i.e. $\partial \hat{C} / \partial z_{L}$ versus $z_{L}$ is very nonlinear at this point) and if the upper part of the interferometer is attached to the suspended electrode, then either the balance beam amplitude must be extremely small, or the balance must be servoed.

5.4 Problems in the slope and path integral methods

The slope at point $\left(\hat{h}, z_{L}\right)$ would be determined in the slope method from the derivative of the $n$ th-order polynomial which provides the best least-squares fit to the $\hat{C}$ versus $z_{L}$ data. If $\hat{C}$ varied linearly with $z_{L}$ and the values of $\hat{C}$ fluctuated by $\pm 1 \mathrm{ppm}$ due to temperature variations and dynamical measurement errors, then the uncertainty in the slope for a $\Delta z_{L}=2.5 \mathrm{~cm}$ scan might be \pm 1 or $2 \mathrm{ppm}$ if the upper part of the interferometer was attached to the suspended electrode and \pm 3 to $5 \mathrm{ppm}$ if it was not.

If the curvature of $\hat{C}$ versus $z_{L}$ is large then, presumably, the uncertainty in the slope of the nth-order polyminal increases. Changes in shape of the $\hat{C}$ versus $z_{L}$ function during a scan would require different polynomial fits over each region - further increasing the slope uncertainties because $\Delta z_{L}$ would be smaller for each region. The great sensitivity of $\hat{C}$ to the shapes of the four electrode surfaces may preclude the use of the slope method. 
In the path integral method it is the difference in capacitance at the two endpoints of the scan that is important. There might be a $\pm 1 \mathrm{ppm}$ uncertainty at each endpoint due to dynamical measurement errors and temperature fluctuations. The uncertainty in $\Delta z_{L}$ between the endpoints of the path integral for $2.5 \mathrm{~cm}$ scans would be \pm 1.5 to $2 \mathrm{ppm}$ if the upper part of the interferometer was attached to the suspended electrode and \pm 3 to $5 \mathrm{ppm}$ if it was not.

$\hat{F}_{Z}$ does not have to be measured at the endpoints of the $z_{L}$ scan if $\hat{F}_{z}$ varies slowly enough with $z_{L}$ so that it can be extrapolated, or interpolated, to the endpoint values. The path integral method will still work if $\hat{C}$ versus $z_{L}$ has large curvatures and shape changes as long as enough $\hat{F}_{Z}$ measurements are taken. The number of $\hat{F}_{Z}$ measurements needed for the path integral depends upon the extent of the $\hat{F}_{Z}$ variations.

$A \pm 1 \mathrm{ppm}$ static systematic error and a $+1 \mathrm{ppm}$ dynamic systematic error could occur in the $\hat{F}_{Z}$ determinations - in addition to a $\pm 1 \mathrm{ppm}$ random error in $\mathrm{mg}$ at $\mathrm{V}=4.02 \mathrm{kV}$.

Only the vertical component of the path integral is measured. The horizontal components due to relative electrode tilts and displacements are unknown. Also, the capacitance is measured at low voltages, and is thus smaller than what it would have been at high voltages if horizontal forces are present.

The errors mentioned in this section might lead to a comparison of the SI volt with the NBS volt to within an uncertainty of \pm 3 or 4 ppm. This is only an estimate. Experience gained with actual data might increase or decrease the uncertainty. 


\section{ACKNOWLEDGEMENTS}

The author gratefully acknowledges the invaluable assistance of R. D. Cutkosky who contributed many hours of discussion about the physical aspects of the problem. He was the vital link with reality. R. S. Davis devoted long hours of discussion on how balances work, critically read the manuscript, and provided the yeoman task of verifying the equations. E. R. Williams also critically read the manuscript, and along with W. D. Phillips, provided many key suggestions - particularly about the path integrals. The author also wishes to thank Mrs. B. L. Main, who has so carefully and cheerfully prepared the manuscript for publication. 
References

[1] Goldstein, H., Classical Mechanics, pp. 14-18 (Addison-Wesley Publishing Co., Inc., Reading, MA., 1950).

[2] Wells, D.A., Theory and Problems of Lagrangian Dynamics, pp. 58-62 (Schaum Publishing Co., New York, N.Y., 1967).

[3] Taylor, B. N., Is the Present Realization of the Absolute Ampere in Error?, Metrologia 12, 81-83 (1976).

[4] Almer, H. E., National Bureau of Standards, One Kilogram Balance, NBS No. 2, J. Res. Nat. Bur. Stand. (U.S.), 76C (Phys. and Chem.), No. 1, 1-10 (Jan. 1972).

[5] Cutkosky, R. D., Capacitance Bridge -- NBS Type 2, Nat. Bur. Stand. (U.S.), Report 7103, 10 pages (March 1961).

[6] Cutkosky, R. D., New NBS Measurements of the Absolute Farad and Ohm, IEEE Trans. IM-23 , No. 4, 305-309 (Dec. 1974).

[7] Childers, C. B., Dziuba, R. F., and Lee, L. H., A Resistive Ratio Standard for Measuring Direct Voltages to $10 \mathrm{kV}$, IEEE Trans. IM-25, No. 4, 505-508 (Dec. 1976).

[8] Kusters, N. L. and Petersons, 0., The Voltage Coefficients of Precision Capacitors, IEEE Trans. IM-11, No. 4, 300-318 (Dec. 1962). 
[9] Hodsman, G. F. and Chappel1, F. A., The Effective Radius of Curvature of Knife Edges, J. Sci. Instr. 29, 330-332 (0ct. 1952).

[10] Smythe, W. R., Static and Dynamic Electricity, pp. 74-76 (McGraw-Hill Publishing Co., Inc., New York, N.Y., 1939).

[11] McLachlan, N.W., Ordinary Non-Linear Differential Equations in Engineering and Physical Sciences, pp. 113-144 (Clarendon Press, 0xford, England, 1956).

[12] Abramowitz, M. and Stegun, I. A., Handbook of Mathematical Functions, pp. 68, 87, 722-743 (Dover Publications, Inc., New York, N.Y., 1968).

[13] Murphy, G. M., Ordinary Differential Equations and Their Solutions, pp. 82-85, 311-316 (D. Van Nostrand Co., Inc., Princeton, New Jersey, 1960). 
The capacitance per unit length, $T$, of three concentric, infinitely long, right circular cylinders is

$$
T=2 \pi \varepsilon_{0} / \ln \left(R_{S}^{I} / r_{C}^{I}\right)+2 \pi \varepsilon_{0} / \ln \left(r_{C}^{0} / R_{S}^{0}\right)
$$

where $R_{S}^{I}, R_{S}^{0}, r_{C}^{I}$ and $r_{C}^{0}$ are shown in Figure 2 - with their values listed in Table 1 , and $\varepsilon_{0}=1 /\left(\mu_{0} c^{2}\right)$, with $\mu_{0}=4 \pi \times 10^{-7} \mathrm{H} / \mathrm{m}$ and $c=$ $2.99792458 \times 10^{8} \mathrm{~m} / \mathrm{s}$. Using these values in Equation $A-1$ one obtains $T=T^{I}+T^{0}=6.0686879 \times 10^{-10} \mathrm{~F} / \mathrm{m}+6.0511887 \times 10^{-10} \mathrm{~F} / \mathrm{m}$.

Smythe [10] has derived an expression for infinitely long right circular cylinders that are radially displaced a distance $d$ from their symmetry axes:

$$
\begin{aligned}
T= & 2 \pi \varepsilon_{0} / \cosh ^{-1}\left\{\left[\left(r_{C}^{I}\right)^{2}+\left(R_{S}^{I}\right)^{2}-d^{2}\right] /\left(2 r_{C}^{I} R_{S}^{I}\right)\right\} \\
& +2 \pi \varepsilon_{0} / \cosh ^{-1}\left\{\left[\left(R_{S}^{0}\right)^{2}+\left(r_{C}^{0}\right)^{2}-d^{2}\right] /\left(2 r_{C}^{0} R_{S}^{0}\right)\right\}
\end{aligned}
$$

where $d^{2}=d_{x}^{2}+d_{y}^{2}$. Using the approximations [12]

$$
\begin{aligned}
& \cosh ^{-1}(u)=\ln \left(u+\sqrt{u^{2}-1}\right) \text { and } \\
& \ln (u)=2\left\{(u-1) /(u+1)+(1 / 3)[(u-1) /(u+1)]^{3}+(1 / 5)[(u-1) /(u+1)]^{5}+\ldots\right\},
\end{aligned}
$$


Dr. F. K. Harris has numerically computed $T$ for small values of $d$, obtaining the result

$$
T(d)=\left[T^{I}(d=0)+\Upsilon^{0}(d=0)\right]\left[1+\left(6.5 \times 10^{-9}\right) d^{2}\right]
$$

where $d$ is now in $\mu \mathrm{m}$ rather than $\mathrm{cm}$. At $\mathrm{d}=0$, Equation $\mathrm{A}-3$ yields $\tau^{I}(d=0)+\tau^{0}(d=0)=6.06868392 \times 10^{-10} \mathrm{~F} / \mathrm{m}+6.05119039 \times 10^{-10} \mathrm{~F} / \mathrm{m}$. These values differ slightly from those obtained from Equation A-1, but it is the change of $T$ with $d$ that concerns us, not the magnitude of $T$.

The radii $R_{S}^{I}, R_{S}^{0}, r_{C}^{I}$ and $r_{C}^{0}$ were chosen so that $T^{I}(d=0) \approx$ $\mathrm{T}^{0}(\mathrm{~d}=0) \approx 6.06 \times 10^{-10} \mathrm{~F} / \mathrm{m}(6.06 \mathrm{pF} / \mathrm{cm})$; therefore,

$$
T(\mathrm{~d})=(12.12 \mathrm{pF} / \mathrm{cm})\left[1+\left(6.5 \times 10^{-9}\right) \mathrm{d}^{2}\right]
$$

This equation is for infinitely long cylinders with radially directed electric field lines. This is not the case for Figure 1, where fringe fields occur. However, given sufficient electrode overlap $L_{0}$, the fringe fields of the suspended and carriage electrodes do not interact, so that $T=12.12 \mathrm{pF} / \mathrm{cm}$ remains valid - and has been experimentally verified.

The capacitance, however, is not equal to $T L_{0}$ because of the fringe fields. A magnitude calibration point (chosen to be $100 \mathrm{pF}$ at $L_{0}=10 \mathrm{~cm}$ and $d=0$ ) is needed to account for fringing, yielding the equation 


$$
C\left(d, z_{\ell}\right)=\left[C\left(z_{j}\right)-(12.12 \mathrm{pF} / \mathrm{cm})\left(z_{\ell}-z_{j}\right)\right]\left[1+\left(6.5 \times 10^{-9}\right) d^{2}\right]
$$

where $z_{i}$ and $z_{\ell}$ are the laser interferometer readings at an initial point $i$ and at a point $\ell$ respectively. Subtracting $z_{j}$ from each point $z_{\ell}$ (thus yielding a relative vertical displacement value $z_{L}=$ $\left.z_{\ell}-z_{j}\right)$, and choosing the capacitance magnitude calibration point as the value for $z_{i}$ (where $z_{L}=0$ ) gives

$$
C\left(d, z_{L}\right)=C\left(z_{L}\right)\left[1+\left(6.5 \times 10^{-9}\right) d^{2}\right]
$$

[C in $\mathrm{pF} ; \mathrm{z}_{\mathrm{L}}$ in $\mathrm{cm} ; \mathrm{d}$ in $\left.\mu \mathrm{m}\right]$

$$
\begin{aligned}
\text { where } d^{2} & =d_{x}^{2}+q_{y}^{2} \text { and } \\
c\left(z_{L}\right) & =\left[100 p F-(12.12 p F / c m) z_{L}\right] .
\end{aligned}
$$

The displacement, $D_{y}$, for the suspended electrode, using Figures 2-4, is

$$
D_{y}=10^{4}\left(L_{A}-L_{A} \cos \beta_{y}-\rho_{p} \sin \beta_{y}-\rho_{P} \sin \gamma_{y}\right)+10^{4} \rho_{C} \beta_{y}
$$

$\left[D_{y}\right.$ in $\mu \mathrm{m} ; L_{A}, \rho_{P}, \rho_{C}$ in $\mathrm{cm} ; \beta_{y}, \gamma_{y}$ in rad $]$.

We will only need $D_{y}$ to hundredths of micrometers, so $D_{y} \approx 10^{4} L_{A}\left(1-\cos \beta_{y}\right)$, and for small $\beta_{y}$ 


$$
D_{y}=10^{4}\left(L_{A} / 2\right) B_{y}^{2}
$$

and

$$
C\left(D_{y}, z_{L}\right)=C\left(z_{L}\right)\left[1+\left(6.5 \times 10^{-9}\right) D_{y}^{2}\right]
$$

[C in pF; $z_{L}, L_{A}$ in cm; $D_{y}$ in $\mu \mathrm{m} ; B_{y}$ in $\left.\mathrm{rad}\right]$. 


\section{APPENDIX B}

\section{CAPACITANCE OF TILTED CYLINDRICAL ELECTRODES}

There are at least seven possible locations in Figure 1 about which the carriage electrodes could pivot - any of the six teflon bearings if they tended to bind on the stainless steel guide rods, and the ball bearing located in crossed $v$-grooves at the top of the elevating shaft. The ball bearing location will be used for the calculations since it is a likely pivot point, and is also geometrically representative of the other pivot points.

Figure 5 shows the outer carriage electrode tilted about the ball bearing (point 3 ) by the angle $\theta_{y^{*}}$. The figure is drawn in the $y-z$ plane (defined in Figure 2) at $x=0$, where the overlap area $A$ (swept out by the carriage electrode as its rim goes from point 1 to point 2) is at a maximum. This area monotonically decreases to zero as one moves around the electrode surface to the $x-z$ plane at $y=0$, and then increases again to another maximum at $y=-r_{c}^{0}$. The resulting volume is similar to that swept out by the outer carriage electrode when it is radially displaced, except in that case the cross section is rectangular.

The overlap area B is what would have been swept out if the electrode were tilted about point 4 rather than point 3. This area could be used to calculate the magnitude of the capacitance if $B=A$, 
which is equivalent to assuming that $B$ is much greater than the upper part of A for small $\theta_{y}$ :

$$
\left[L_{0} \ell \theta_{y}-(1 / 2) L_{0}^{2} \theta_{y}\right]>>\left[(1 / 2) e r_{C}^{0} \theta_{y}^{2}\right] \text { or } L_{0}\left[\ell-L_{0} / 2\right]>>\left[(1 / 2) e r_{C}^{0} \theta_{y}\right] \text {. }
$$

Letting $L_{0}=10 \mathrm{~cm}$ and $\theta_{y}=4.85 \times 10^{-5} \mathrm{rad}\left(10^{\prime \prime}\right)$ yields $300 \gg 0.009\left(\mathrm{~cm}^{2}\right)$. Therefore the pivot will be transposed to point 4 .

We know from Equations $A-5$ and A-6 of Appendix $A$ how to calculate the capacitance for radial displacements, and could use those results if electrode tilts were expressed as equivalent displacements. Therefore, divide $C_{0}\left(d_{y}, z_{L}\right)$ into infinitesimal sections $c^{0}\left(d_{y}, z_{L}\right) / L_{0}\left(z_{L}\right)$ and sum them to get $c_{t}^{0}\left(\theta_{y}, z_{L}\right)$ :

$$
\begin{aligned}
c c_{t}^{0}\left(\theta_{y}, z_{L}\right)= & {\left[1 / L_{0}\left(z_{L}\right)\right] \int_{\ell^{\prime}\left(z_{L}\right)}^{l} c^{0}\left(d_{y}, z_{L}\right) d \lambda_{t}=} \\
& {\left[c^{0}\left(z_{L}\right) / L_{0}\left(z_{L}\right)\right] \int_{l^{\prime}\left(z_{L}\right)}^{l}\left[1+(0.65) \lambda_{t}^{2} \theta_{y}^{2}\right] d \lambda_{t} }
\end{aligned}
$$

where $d_{y}=10^{4} \lambda_{t} \theta_{y}$ and $\lambda_{t}$ is in $\mathrm{cm}$. Therefore,

$$
c_{t}^{0}\left(\theta_{y}, z_{L}\right)=c^{0}\left(z_{L}\right)\left\{1+(0.217)\left[e^{3}-e^{\prime 3}\left(z_{L}\right) \theta_{y}^{2} / L_{0}\left(z_{L}\right)\right]\right\}
$$

[C in pF; $\ell, \ell^{\prime}, L_{0}, z_{L}$ in $\mathrm{cm} ; \theta_{y}$ in $\mathrm{rad}$ ]. 
This equation could be used to compute the capacitance due to a tilted outer carriage electrode, but it will instead be reduced to a much simpler form. The centroid of area B, if measured from point 4 , is

$$
\begin{aligned}
& e_{t}\left(z_{L}\right)=\int_{\ell^{\prime}\left(z_{L}\right)}^{l} \lambda_{t} d A / \int_{l^{\prime}\left(z_{L}\right)}^{l} d A= \\
& \int_{\ell^{\prime}\left(z_{L}\right)}^{l} \lambda_{t^{\circ}} \lambda_{t^{\theta} y}\left(d \lambda_{t}\right) / \int_{l^{\prime}\left(z_{L}\right)}^{l} \lambda_{t} \theta_{y}\left(d \lambda_{t}\right)= \\
& (2 / 3)\left\{\left[l^{3}-l^{\prime 3}\left(z_{L}\right)\right] /\left[l^{2}-l^{\prime 2}\left(z_{L}\right)\right]\right\}
\end{aligned}
$$

$\left[\ell_{t}, \ell, \ell^{\prime}\right.$ in $\left.\mathrm{cm}\right]$

or, if measured from the gravitational horizon of the balance beam, is

$$
L_{t}\left(z_{L}\right)=L+\ell^{\prime}\left(z_{L}\right)-e_{t}\left(z_{L}\right)=L+\ell-L_{0}\left(z_{L}\right)-e_{t}\left(z_{L}\right)
$$

Table 2 lists $L_{t}$ versus $z_{L}$ for an initial value of $C=100 p F$ and $L_{0}$ $=10 \mathrm{~cm}$. Note that at the most $L_{t}$ varies from $L_{d}$ by $0.5 \%$, where $L_{d}=$ $L_{D}=L-L_{0} / 2$. Therefore, let $L_{t}=L_{d}$, so $L+\ell-L_{0}-\ell_{t}=L-$ $L_{0} / 2$, or

$$
\ell_{t}\left(z_{L}\right)=\ell-L_{0}\left(z_{L}\right) / 2
$$


(This equation assumes that $L_{0}>r^{0} c^{\theta} y$, which for $\theta_{y}=4.85 \times 10^{-5} \mathrm{rad}$ (10") is $10 \mathrm{~cm} \gg(10.74 \mathrm{~cm})\left(4.85 \times 10^{-5} \mathrm{rad}\right)=5.21 \times 10^{-4} \mathrm{~cm}$. $)$

If Equation B-2 is substituted into Equation B-1, then

$$
c_{t}^{0}\left(\theta_{y}, z_{L}\right)=c^{0}\left(z_{L}\right)\left\{1+(0.217)\left[(3 / 2)\left(\ell-\ell^{\prime}\right)\left(\ell+\ell^{\prime}\right) / L_{0}\right] \ell_{t} \theta_{y}^{2}\right\} .
$$

Noting that $L_{0}=\ell-\ell^{\prime}, c^{0}\left(z_{L}\right)=C^{I}\left(z_{L}\right)$, and using Equation $B-4$ :

$$
C_{t}\left(\theta, z_{L}\right)=C\left(z_{L}\right)\left(1+(0.65)\left[2-L_{0}\left(z_{L}\right) / 2\right]^{2} \theta^{2}\right\}
$$

Comparing Equations $\mathrm{B}-5$ and $\mathrm{A}-5$ we see that the capacitance due to tilting both carriage electrodes can be written as an equivalent radial displacement, $d_{t}$, of the centroid $\ell_{t}$ of the area $B$, where

$$
d_{t}=10^{4}\left[l-L_{0}\left(z_{L}\right) / 2\right] \theta
$$

$\left[d_{t}\right.$ in $\mu m ; l, L_{0}, z_{L}$ in $\mathrm{cm} ; \theta$ in $\left.\mathrm{rad}\right]$,

$$
\theta^{2}=\theta_{x}^{2}+\theta_{y}^{2} \text {, and } C\left(z_{L}\right) \text { is defined by Equation } A-6 \text {. }
$$

An exactly analogous derivation for the capacitance due to the suspended electrode pivoting about point $P$ of Figure 2 yields the results

$$
C_{T}\left(\gamma, z_{L}\right)=C\left(z_{L}\right)\left\{1+(0.65)\left[L-L_{0}\left(z_{L}\right) / 2\right]^{2} \gamma^{2}\right\}
$$




$$
\text { and } d_{T}=10^{4}\left[L-L_{0}\left(z_{L}\right) / 2\right] \gamma
$$

[d $\mathrm{d}_{\mathrm{T}}$ in $\mu \mathrm{m} ; \mathrm{L}, \mathrm{L}_{0}, z_{\mathrm{L}}$ in $\mathrm{cm}$; $C$ in $\mathrm{pF}$; $\gamma$ in $\left.\mathrm{rad}\right]$, where $\gamma^{2}=\gamma_{x}^{2}+\gamma_{y}^{2}$, and $C\left(z_{L}\right)$ is again defined by Equation $A-6$. 


\section{APPENDIX C}

VARIATIONS AND UNCERTAINTIES OF $z_{L}$ AND $C$

a) The laser interferometer alignment.

The $z_{L}$ axis is defined by the direction of the laser interferometer, whereas the $z$ axis (which is also the direction of the combined electrodes symmetry axis) is parallel to the gravitational field. How well must the $z_{L}$ axis be aligned with the $z$ axis in order for $\Delta z_{L}$ to be within 1 part in $10^{7}$ of $\Delta z$ ?

$$
\begin{aligned}
& \Delta z=\left(\Delta z_{L}\right)_{z}=\Delta z_{L} \cos \phi_{L}=\Delta z_{L}\left(1-\phi_{L}^{2} / 2+\ldots\right) \\
& \therefore \phi_{L}^{2} / 2=1 \times 10^{-7} \text {, so } \\
& \phi_{L}=4.47 \times 10^{-4} \mathrm{rad}\left(92^{\prime \prime}\right) .
\end{aligned}
$$

This should not be difficult to attain.

b) The uncertainty in measuring $\Delta z_{L}$.

The laser interferometer system can measure distances with a reproducibility of $\delta_{L}= \pm 0.025 \mu \mathrm{m}$. How large must $\Delta z_{L}$ be in order for it to be known to within $1 \mathrm{ppm}$ ?

$$
\delta_{L} / \Delta z_{L}=\sqrt{2}( \pm 0.025 \mu \mathrm{m}) / \Delta z_{L}= \pm 1 \times 10^{-6} \text {, so } \Delta z_{L}=3.5 \mathrm{~cm} \text {. }
$$


c) The variation of $z_{L}$ for a freely-swinging balance beam.

It is estimated that the values of $\beta_{y}$ were $\pm 2.4 \times 10^{-3}$ rad for the turning points of the balance beam when the $1 \mu \mathrm{g}$ reproducibility was achieved with the voltage off. Therefore

$$
\begin{aligned}
& \Delta z_{L} \approx-L_{A} \sin \beta y^{\approx}-L_{A} \beta y=-(7.5 \mathrm{~cm})\left( \pm 2.4 \times 10^{-3} \mathrm{rad}\right) \\
& \Delta z_{L}= \pm 0.018 \mathrm{~cm}( \pm 180 \mu \mathrm{m}) .
\end{aligned}
$$

d) The variation of $C$ for a freely-swinging balance beam. Using Equation 2-12 and the result of part c:

$$
\begin{aligned}
\left(\partial C / \partial z_{L}\right) & =-12.12 \mathrm{pF} / \mathrm{cm}\left[1+\left(6.5 \times 10^{-9}\right) \mathrm{h}^{2}\right] \approx-12.12 \mathrm{pF} / \mathrm{cm} \\
\delta C / C & =\left(\partial C / \partial z_{L}\right) \Delta z_{L} / C=(-12.12 \mathrm{pF} / \mathrm{cm})\left( \pm 1.8 \times 10^{-2} \mathrm{~cm}\right) / 50 \mathrm{pF} \\
\delta C / C & = \pm 4.36 \times 10^{-3}= \pm 0.44 \% \text { at } C=50 \mathrm{pF} .
\end{aligned}
$$

The value of $C=50 \mathrm{pF}$ was chosen because it, along with $C=100 \mathrm{pF}$, will be the values compared with reference capacitors. Clearly the balance beam cannot swing during the capacitance measurements. It will either have to be mechanically braked or electrically servoed to gravitational horizontal.

e) The variation of $z_{L}$ and $C$ for a servoed balance beam. How well must $\beta_{y}$ be servoed for $\delta C / C= \pm 1 \times 10^{-6}$

$$
\begin{aligned}
& \Delta z_{L}=\left( \pm 1 \times 10^{-6}\right) \mathrm{c} /\left(\partial \mathrm{C} / \partial \mathrm{z}_{\mathrm{L}}\right)=\left( \pm 1 \times 10^{-6}\right)(50 \mathrm{pF}) /(-12.12 \mathrm{pF} / \mathrm{cm}) \\
& \Delta z_{L}= \pm 4.12 \times 10^{-6} \mathrm{~cm}( \pm 0.041 \mu \mathrm{m}) .
\end{aligned}
$$


The capacitance will change by 1 ppm if the electrode overlap $L_{0}$ changes by a tenth of a wavelength of blue light. The laser interferometer could monitor this via $z_{L}$ however (if the upper part were attached to the suspended electrode system) because $\delta_{L}=$ $\pm 0.025 \mu \mathrm{m}$.

$$
\beta_{y}=\Delta z_{L} / L_{A}=\left( \pm 4.12 \times 10^{-6} \mathrm{~cm}\right) /(7.5 \mathrm{~cm})= \pm 5.50 \times 10^{-7} \mathrm{rad}\left( \pm 0.11^{\prime \prime}\right) .
$$

Therefore $\beta_{y}$ would have to be servoed to \pm 0.11 " in order to measure the capacitance at $50 \mathrm{pF}$ to within $\pm 1 \mathrm{ppm}$.

f) The electrode shape-dependence of $C$.

We can use Equation A-1 to find the capacitance per unit length, $T^{\prime}$, if the average radius of any electrode surface varies from the value for a right circular cylinder:

$$
\begin{gathered}
T^{\prime}=2 \pi \varepsilon_{0} /\left\{\ln \left[R_{S}^{I}\left(1+\delta_{S}^{I}\right) / r_{C}^{I}\left(1+\delta_{C}^{I}\right)\right]\right\}+ \\
2 \pi \varepsilon_{0} /\left\{\ln \left[r_{C}^{0}\left(1+\delta_{C}^{0}\right) / R_{S}^{0}\left(1+\delta_{S}^{0}\right]\right\}\right.
\end{gathered}
$$

where $\delta_{C}^{I}=\Delta r_{C}^{I} / r_{C}^{I}, \delta_{S}^{I}=\Delta R_{S}^{I} / R_{S}^{I}$, etc. Using the expansion

$$
\ln (1+u)=u-u^{2} / 2+u^{3} / 3 \ldots \ldots u,
$$

we obtain the result 


$$
\begin{aligned}
& \Delta T=T^{\prime}-T=2 \pi \varepsilon_{0}\left(\delta_{C}^{I}-\delta_{S}^{I}\right) /\left(\ln R_{S}^{I} / r_{C}^{I}\right)^{2}+ \\
& 2 \pi \varepsilon_{0}\left(\delta_{S}^{0}-\delta_{C}^{0}\right) /\left(\ln r{ }_{C}^{0} R_{S}^{0}\right)^{2},
\end{aligned}
$$

where $T=12.12 \mathrm{pF} / \mathrm{cm}$. Using the values from Table 1 :

$$
\begin{aligned}
\delta C / C=\Delta T / T= & \left(4.59 \times 10^{-2}\right)\left[(119.0)\left(\delta_{C}^{I}-\delta_{S}^{I}\right)+\right. \\
& \left.(118.3)\left(\delta_{S}^{0}-\delta_{C}^{0}\right)\right] .
\end{aligned}
$$

If the outer surface of the suspended electrode was lapped more at the rim than in the middle, then it would deviate from a right circular cylinder. Assume this surface has a slope of $1 \mu \mathrm{m} / \mathrm{cm}$ over the last $10 \mathrm{~cm}$, so that the radius at the rim is $10 \mu \mathrm{m}$ smaller than $R_{S}^{0}$. The average value of $\delta_{S}^{0}$ depends upon the electrode overlap, and has the value $-5.10 \times 10^{-5}$ at $\mathrm{C}=100 \mathrm{pF}$. Let the other three electrodes be right cylinders, so $\delta_{C}^{I}=\delta_{S}^{I}=\delta_{C}^{0}=0$. Then $\delta C / C=-277 \mathrm{ppm}$ at $C=$ $100 \mathrm{pF}$ and $\delta C / C=-388 \mathrm{ppm}$ at $C=51.54 \mathrm{pF}$. These variations from the values that the capacitance would have for right cylinders are linear with $z_{L}$ over the $\Delta z_{L}=4 \mathrm{~cm}$ span. If the slope was not a constant 1 $\mu \mathrm{m} / \mathrm{cm}$, then a plot of $C$ versus $z_{L}$ would have curvature. These variations of $C$ are real effects, not errors.

Let all the electrode surfaces be lapped more near their rims, with constant slopes of $1 \mu \mathrm{m} / \mathrm{cm}$ over the 1 ast $10 \mathrm{~cm}$. Then $\delta C / C=$ $-1139 \mathrm{ppm}$ at $\mathrm{C}=100 \mathrm{pF}$ and $\delta \mathrm{C} / \mathrm{C}=-1595 \mathrm{ppm}$ at $\mathrm{C}=51.54 \mathrm{pF}$. A plot of $C$ versus $z_{L}$ would be a straight line only if all four electrode surfaces had constant slopes. 
Let all four electrode surfaces be right circular cylinders, but assume there is a bulge extending around the outer surface of the suspended electrode. Let the bulge be symmetrically shaped with a $5 \mu \mathrm{m}$ height and a $4 \mathrm{~cm}$ base, and place the peak $8 \mathrm{~cm}$ from the electrode rim. Then $\delta C / C$ varies from about $37 \mathrm{ppm}$ to about $35 \mathrm{ppm}$ in the $\Delta z_{L}=$ $2 \mathrm{~cm}$ distance between $\mathrm{C}=100 \mathrm{pF}$ and $\mathrm{C}=75.76 \mathrm{pF}$, and then decreases to about $0 \mathrm{ppm}$ in the $\Delta \mathrm{z}_{\mathrm{L}}=2 \mathrm{~cm}$ span between $\mathrm{C}=75.76 \mathrm{pF}$ and $\mathrm{C}=$ $51.54 \mathrm{pF}$.

These examples demonstrate that the value of $C$, at any particular value of $z_{L}$, is very sensitive to the shapes of the electrode surfaces. If the average radius of any surface, for a given electrode overlap, changes by $\pm 1 \mu \mathrm{m}$ from the value for a right circular cyl inder, then $\delta C / C \approx \pm 54 \mathrm{ppm}$. 


\section{APPENDIX D}

\section{VARIATIONS OF C WITH TEMPERATURE}

a) Variation of $C$ with uniform temperature changes.

The corner cube mirror in Figure 1 is mounted near the carriage electrode rims, so it can monitor the carriage electrodes thermal expansions. The upper part of the laser interferometer, however, is about $40 \mathrm{~cm}$ from the rim of the suspended electrode, so the electrode overlap $L_{0}$ could change due to expansion of the suspended electrode system below the upper part of the interferometer and not be monitored via the $z_{L}$ measurement. (Only the overlap change due to the carriage electrodes expansions would be monitored.) The upper part of the suspension system is essentially temperature compensated, whether or not the upper part of the interferometer is attached, to it, because the coefficients of thermal expansion of the aluminum, brass and stainless steel guide rod components are all about $\alpha=2 \times 10^{-5} / \mathrm{C}^{\circ}$. Thus

$$
\delta z_{L} / \Delta T=L \propto=(40 \mathrm{~cm})\left(2 \times 10^{-5} / \mathrm{C}^{\circ}\right)\left({ }^{\circ} \mathrm{C} / 10^{3} \mathrm{~m}^{\circ} \mathrm{C}\right)=8 \times 10^{-7} \mathrm{~cm} / \mathrm{m}^{\circ} \mathrm{C} .
$$

Using a result from part e of Appendix C:

$$
\delta \mathrm{C} / \mathrm{C}=\left(8 \times 10^{-7} \mathrm{~cm} / \mathrm{m}^{\circ} \mathrm{C}\right)\left(1 \mathrm{~m}^{\circ} \mathrm{C}\right)\left(\mathrm{ppm} / 4.12 \times 10^{-6} \mathrm{~cm}\right)=0.19 \mathrm{ppm}
$$


for a $1 \mathrm{~m}^{\circ} \mathrm{C}$ temperature increase at $\mathrm{C}=50 \mathrm{pF}$.

This result is for a uniform temperature increase everywhere in the 0 -ring sealed container. If the temperature varies during data collection, then the capacitance measurements will account for the $\delta C / C$ changes, but the $z_{L}$ measurements will be in error.

b) Variation of $\mathrm{C}$ as the carriage moves through a vertical temperature gradient.

A higher temperature is maintained at the top of the container to minimize convection currents, which would affect the balance measurements. Assuming a constant temperature gradient of $1 \mathrm{~m}^{\circ} \mathrm{C} / \mathrm{cm}$, the average temperature change of the carriage electrodes is $\Delta T=-4 \mathrm{~m}^{\circ} \mathrm{C}$ during the $\Delta \mathrm{z}_{\mathrm{L}} \approx 4 \mathrm{~cm}$ carriage translation from a capacitance value of $\mathrm{C}=100 \mathrm{pF}$ to $\mathrm{C}=50 \mathrm{pF}$.

The ${ }^{l_{C}}=25 \mathrm{~cm}$ heights of the carriage electrodes have thus changed by

$$
{ }^{\Delta l} \mathrm{C}={ }^{\ell} \mathrm{C}^{\alpha \Delta \mathrm{T}}=(25 \mathrm{~cm})\left(2 \times 10^{-8} / \mathrm{m}^{\circ} \mathrm{C}\right)\left(-4 \mathrm{~m}^{\circ} \mathrm{C}\right)=-2 \times 10^{-6} \mathrm{~cm} \text {, }
$$

giving the result

$$
\delta C / C=\left(-2 \times 10^{-6} \mathrm{~cm}\right)\left(\mathrm{ppm} / 4.12 \times 10^{-6} \mathrm{~cm}\right)=-0.49 \mathrm{ppm}
$$

for a constant temperature gradient of $1 \mathrm{~m}^{\circ} \mathrm{C} / \mathrm{cm}$ at $\mathrm{C}=50 \mathrm{pF}$. 
This is a real effect, not an error, because the ${ }_{C}$ change is correctly monitored via the $z_{L}$ measurement. There would be an error, however, if the temperature gradient changed during data collection because of the effect presented in part a.

C) Variation of $C$ for a radial temperature gradient across the electrodes.

Let the inner carriage electrode be $1 \mathrm{~m}^{\circ} \mathrm{C}$ warmer than the suspended electrode, and the outer carriage electrode be $1 \mathrm{~m}^{\circ} \mathrm{C}$ cooler than the suspended electrode. Then $\delta_{C}^{I}=\Delta r_{C}^{I} / r_{C}^{I}=\alpha \Delta T=2 \times 10^{-8}, \delta_{S}^{I}=$ $\delta_{S}^{0}=0$, and $\delta_{C}^{0}=-\delta_{C}^{I}$. Using these values in Equation $C-1$ yields

$$
\delta \mathrm{C} / \mathrm{C}=0.22 \mathrm{ppm}
$$

for a $-2 \mathrm{~m}^{\circ} \mathrm{C}$ radial temperature gradient across the electrodes. There would be errors if this temperature gradient changed during data collection because the values of $C$ would change for a given $z_{L}$ value. Also, the lengths $\ell_{C}^{I}$ and $\ell_{C}^{0}$ would change by different amounts, so the corner cube mirror would not correctly monitor individual variations of the inner and outer electrode overlaps, resulting in errors of the type presented in part $a$. 


\section{DERIVATION OF THE FORCE EQUATIONS}

The electrical potential energy, $U^{e}$, stored in the electric field of a capacitor is $U^{e}=(1 / 2) C V^{2}$, and the change in $U^{e}$ from an initial state $i$ to a state $j$, using conservation of energy, is

$$
\Delta U^{e}=(1 / 2) c_{j} v_{j}^{2}-(1 / 2) c_{i} v_{i}^{2}=-\int_{l_{i}}^{l_{j}} \vec{F} \cdot d \vec{l}+\int_{q_{i}}^{q_{j}} V d q
$$

[U in $N \cdot m$; $C$ in $F$; $V$ in $V ; F$ in $N$; $\quad$ in $m ; q$ in $C$ ]

where $F$ is the electrical force and $q$ is the charge on the electrodes.

Using the coordinate system of Figure 7 , and anticipating that

$F_{h_{x}}^{0}=F_{h_{x}}^{I}$ and $F_{h_{y}}^{0}=F_{h_{y}}^{I}$ (because $h_{x}^{0}=h_{x}^{I}$ and $h_{y}^{0}=h_{y}^{I}$ ), the differential of Equation E-1 becomes

$$
d u^{e}=-2 F_{h_{x}} d h_{x}-2 F_{h_{y}} d h_{y}-F_{z_{L}} d z_{L}+V d q
$$

but

$$
\begin{aligned}
& d U^{e}=\left(\partial u^{e} / \partial h_{x}\right) d h_{x}+\left(\partial u^{e} / \partial h_{y}\right) d h_{y}+\left(\partial U^{e} / \partial z_{L}\right) d z_{L} \\
& d q=\left(\partial q / \partial h_{x}\right) d h_{x}+\left(\partial q / \partial h_{y}\right) d h_{y}+\left(\partial q / \partial z_{L}\right) d z_{L} \cdot
\end{aligned}
$$


Substituting these equations into Equation $E-2$, and realizing that $h_{x}$, $h_{y}$ and $z_{L}$ are independent variables - so that the equation must be satisfied for each variable - we obtain:

$$
\begin{aligned}
& \left(\partial u^{e} / \partial h_{x}\right)=-2 F_{h_{x}}+v\left(\partial q / \partial h_{x}\right) \\
& \left(\partial u^{e} / \partial h_{y}\right)=-2 F_{h_{y}}+v\left(\partial q / \partial h_{y}\right) \\
& \left(\partial u^{e} / \partial z_{L}\right)=-F_{z_{L}}+v\left(\partial q / \partial z_{L}\right) .
\end{aligned}
$$

Using $U^{e}=(1 / 2) C V^{2}$ and $q=C V$ in these three equations thus yields

$$
\begin{aligned}
& F_{h_{x}}=(1 / 4) v^{2}\left[\partial c\left(h, z_{L}\right) / \partial h_{x}\right]_{h_{y}, z_{L}} \\
& F_{h_{y}}=(1 / 4) v^{2}\left[\partial c\left(h, z_{L}\right) / \partial h_{y}\right]_{h_{x}, z_{L}} \\
& F_{z_{L}}=(1 / 2) v^{2}\left[\partial C\left(h, z_{L}\right) / \partial z_{L}\right]_{h_{x}, h_{y}}
\end{aligned}
$$

[F in $N ; V$ in $V ; C$ in $F ; h, z_{L}$ in $\left.m\right]$. 
COORDINATES OF POINTS UNDERGOING ONLY $\gamma_{y}$ and $\beta_{y}$ MOTIONS

This appendix lists the $y$ and $z$ coordinates of the six points, shown in Figure 2, which locate the positions of the gravitational and electrical forces when only $\gamma_{y}$ and $\beta_{y}$ motions occur. Figures $3,4,8$ and 9 are also needed to determine these coordinates. The units are given at the end of the list.

$$
\begin{aligned}
& y_{1}=-L_{A} \cos \beta_{y}-\rho_{p} \sin \beta_{y}-\rho_{p} \sin \gamma_{y}+L_{c m} \sin \gamma_{y} \\
& z_{1}=-L_{A} \sin \beta_{y}-\rho_{P} \cos \gamma_{y}+\rho_{P} \cos \beta_{y}+L_{c m} \cos \gamma_{y} \\
& y_{2}=-\ell_{b} \sin \beta y \\
& z_{2}=\ell_{b} \cos \beta_{y} \\
& y_{3}=L_{B} \cos \beta_{y}-\ell_{B} \sin \beta_{y} \\
& z_{3}=L_{B} \sin \beta_{y}+\ell_{B} \cos \beta_{y} \\
& y_{4}=-L_{A} \cos \beta_{y}-\rho_{P} \sin \beta_{y}-\rho_{P} \sin \gamma_{y}+L \sin \gamma_{y} \\
& z_{4}=-L_{A} \sin \beta_{y}-\rho_{P} \cos \gamma_{y}+\rho_{p} \cos \beta_{y}+L \cos \gamma_{y} \\
& y_{5}=-L_{A} \cos \beta_{y}-\rho_{P} \sin \beta_{y}-\rho_{p} \sin \gamma_{y}+L \sin \gamma_{y}+R_{S}^{0} \cos \gamma_{y} \\
& -(1 / 2)\left[L_{0}-R_{S}^{0} \sin \gamma_{y}-L\left(1-\cos \gamma_{y}\right)+r_{C}^{0} \sin \theta_{y}-\ell\left(1-\cos \theta_{y}\right)\right] \tan \gamma_{y} \\
& z_{5}=-L_{A} \sin \beta_{y}-\rho_{p} \cos \gamma_{y}+\rho_{p} \cos \beta_{y}+L \cos \gamma_{y}-R_{S}^{0} \sin \gamma_{y} \\
& -(1 / 2)\left[L_{0}-R_{S}^{0} \sin \gamma_{y}-L\left(1-\cos \gamma_{y}\right)+r_{C}^{0} \sin \theta_{y}-\ell\left(1-\cos \theta_{y}\right)\right] \\
& y_{6}=-L_{A} \cos \beta_{y}-\rho_{P} \sin \beta_{y}-\rho_{P} \sin \gamma_{y}+L \sin \gamma_{y}-R_{S}^{I} \cos \gamma_{y} \\
& -(1 / 2)\left[L_{0}+R \frac{I}{S} \sin \gamma_{y}-L\left(1-\cos \gamma_{y}\right)-r_{C} \sin \theta_{y}-\ell\left(1-\cos \theta_{y}\right)\right] \tan \gamma_{y} \\
& z_{6}=-L_{A} \sin \beta_{y}-\rho_{P} \cos \gamma_{y}+\rho_{P} \cos \beta_{y}+L \cos \gamma_{y}+R_{S} \sin \gamma_{y} \\
& -(1 / 2)\left[L_{0}+R I_{S} \sin \gamma_{y}-L\left(1-\cos \gamma_{y}\right)-r_{C} \sin \theta_{y}-\ell\left(1-\cos \theta_{y}\right)\right]
\end{aligned}
$$

$\left[y, z, L_{A}, \rho_{p}, L_{c m}, \ell_{b}, \ell_{B}, L_{B}, L, L_{0}, l, R_{S}^{0}, R_{S}^{I}, r_{C}^{0}, r_{C}^{I}\right.$ in $c m ; \gamma_{y}, \beta_{y}$, $\theta_{y}$ in $\left.\mathrm{rad}\right]$. 
COORDINATES OF POINTS UNDERGOING ONL $Y \gamma_{x}$ and $\beta_{y}$ MOTIONS

This appendix lists the $x$ and $z$ coordinates of the six points, shown in Figure 2, which locate the positions of the gravitational and electrical forces when only $\gamma_{x}$ and $\beta_{y}$ motions occur. The points 5 and 6 are, however, in the $x-z p l$ ane, rather than the $y-z$ plane. Figures $3,4,8$ and 9 are also needed to determine these coordinates. The $D_{y}$ displacements, due to the $\beta_{y}$ motion, is ignored; so there are no $F_{y}^{0}$ and $F_{y}^{I}$ forces, and the $y$ coordinates are therefore not required. The units are given at the end of the list.

$$
\begin{aligned}
x_{1}= & L_{c m} \sin \gamma_{x} \\
z_{1}= & -L_{A} \sin \beta_{y}+\rho_{P} \cos \beta_{y}+L_{c m} \cos \gamma_{x} \\
x_{2}= & \\
z_{2}= & l_{b} \cos \beta_{y} \\
x_{3}= & \\
z_{3}= & L_{B} \sin \beta_{y}+\ell_{B} \cos \beta_{y} \\
x_{4}= & L \sin \gamma_{x} \\
z_{4}= & -L_{A} \sin \beta_{y}+\rho_{P} \cos \beta_{y}+L \cos \gamma_{x} \\
x_{5}= & L \sin \gamma_{x}+R{ }_{S} \cos \gamma_{x}-(1 / 2)\left[L_{0}-R_{S}^{0} \sin \gamma_{x}-L\left(1-\cos \gamma_{x}\right)\right. \\
& \left.+r_{C}^{0} \sin \theta_{x}-\ell\left(1-\cos \theta_{x}\right)\right] \tan \gamma_{x} \\
z_{5}= & -L_{A} \sin \beta_{y}+\rho_{P} \cos \beta_{y}+L \cos \gamma_{x}-R_{S}^{0} \sin \gamma_{x} \\
& -(1 / 2)\left[L_{0}-R{ }_{S}^{0} \sin \gamma_{x}-L\left(1-\cos \gamma_{x}\right)+r_{C}^{0} \sin \theta_{y}-\ell\left(1-\cos \theta_{x}\right)\right]
\end{aligned}
$$




$$
\begin{aligned}
& x_{6}=L \sin \gamma_{X}-R \frac{I}{\cos \gamma_{X}}-(1 / 2)\left[L_{0}+R I_{S}^{I} \sin \gamma_{X}-L\left(1-\cos \gamma_{X}\right)\right. \\
& \left.-r \frac{I}{C} \sin \theta_{x}-\ell\left(1-\cos \theta_{x}\right)\right] \tan \gamma_{x} \\
& z_{6}=-L_{A} \sin \beta_{y}+\rho_{p} \cos \beta_{y}+L \cos \gamma_{x}+R \frac{I}{S} \sin \gamma_{x} \\
& -(1 / 2)\left[L_{0}+R_{S}^{I} \sin \gamma_{x}-L\left(1-\cos \gamma_{x}\right)-r_{C}^{I} \sin \theta_{x}-\ell\left(1-\cos \theta_{x}\right)\right] \\
& {\left[x, z, L_{A}, \rho_{p}, L_{c m}, \ell_{b}, \ell_{B}, L_{B}, L, L_{0}, \ell, R_{S}^{0}, R_{S}^{I}, r_{C}^{0}, r_{C}^{I} \text { in } \mathrm{cm} ; \gamma_{x}\right. \text {, }} \\
& \left.B_{y}, \theta_{x} \text { in } \mathrm{rad}\right] \text {. }
\end{aligned}
$$

78 


\section{APPENDIX H}

PROOF THAT $h_{x}^{I}=h_{x}^{0}$ AND $h_{y}^{I}=h_{y}^{0}$

Using Appendix $F$ and Figure 2, and recalling that $h_{y}^{0}$ and $d_{y}$ are in $\mu \mathrm{m}$, then the relative displacement, $h_{y}^{0}$, of the suspended electrode and the outer carriage electrode is:

$$
\begin{aligned}
10^{-4} h_{y}^{0}= & \left\{y_{5}-\left(R_{S}^{0}-L_{A}\right)\right\}+\left\{\left(r_{C}^{0}-L_{A}\right)-\left[-L_{A}-10^{-4} d_{y}+r_{C}^{0} \cos \theta_{y}-\ell \sin \theta_{y}\right.\right. \\
& \left.\left.+\left(L_{0}^{0} / 2\right) \tan \theta_{y}\right]\right\}
\end{aligned}
$$

where

$$
L_{0}^{0}=L_{0}-R_{S}^{0} \sin \gamma_{y}-L\left(1-\cos \gamma_{y}\right)+r_{C}^{0} \sin \theta_{y}-\ell\left(1-\cos \theta_{y}\right) .
$$

Using the small angle approximations $\sin \Psi \approx \tan \Psi \approx \Psi$ and $\cos \Psi \approx 1$ $\psi^{2} / 2$, and keeping only the predominant terms, yields the result

$$
h_{y}^{0}=10^{4}\left(L_{A} / 2\right) B_{y}^{2}+10^{4}\left(L-L_{0} / 2\right) \gamma_{y}+10^{4}\left(\ell-L_{0} / 2\right) \theta_{y}+d_{y}
$$

[h, d in $\mu m ; L, \ell, L_{0}, L_{A}$ in $c m ; \beta, \gamma, \theta$ in rad].

This is the same expression as that derived in Section 2.3.

If the expression analogous to Equation $\mathrm{H}-1$ is written for the relative displacement, $h_{y}^{I}$, of the suspended electrode and the inner carriage electrode - and similar approximations are made to this expression - then one finds that $h_{y}^{I}=h_{y}^{0}$. 
The expression for $h_{x}^{0}$ is:

$$
\begin{gathered}
10^{-4} h_{x}^{0}=\left\{x_{5}-R_{S}^{0}\right\}+\left\{r_{C}^{0}-\left[-10^{-4} d_{x}+r_{C}^{0} \cos \theta_{x}-\ell \sin \theta_{x}+\right.\right. \\
\left.\left.\left(L_{0}^{0} / 2\right) \tan \theta_{x}\right]\right\}
\end{gathered}
$$

where

$$
L_{0}^{0}=L_{0}-R_{S}^{0} \operatorname{sim} \gamma_{x}-L\left(1-\cos \gamma_{x}\right)+r_{C}^{0} \sin \theta_{x}-\ell\left(1-\cos \theta_{x}\right) .
$$

Making the same approximations as above, we obtain the equation

$$
h_{x}^{0}=10^{4}\left(L-L_{0} / 2\right) \gamma_{x}+10^{4}\left(\ell-L_{0} / 2\right) \theta_{x}+d_{x}
$$

[h,d in $\mu \mathrm{m} ; \mathrm{L}, \ell, L_{0}$ in $\mathrm{cm} ; \gamma, \theta$ in $\left.\mathrm{rad}\right]$,

which is the same expression as Equation 2-9. A similar procedure for $h_{x}^{I}$ gives the result $h_{x}^{I}=h_{x}^{0}$. 
GENERALIZED TORQUES FOR $\gamma_{y}$ AND $\beta_{y}$ MOTIONS ONLY

a) Derivation of $Q_{\gamma_{y}}$.

Using Equations 3-3 and 3-4:

$$
\begin{array}{r}
q_{\gamma_{y}}=M_{A} g\left(\partial z_{1} / \partial \gamma_{y}\right)+M_{b} g\left(\partial z_{2} / \partial \gamma_{y}\right)+M_{B} g\left(\partial z_{3} / \partial \gamma_{y}\right) \\
+F_{z}\left(\partial z_{4} / \partial \gamma_{y}\right)+F_{y}^{0}\left(\partial y_{5} / \partial \gamma_{y}\right)+F_{y}^{I}\left(\partial y_{6} / \partial \gamma_{y}\right) .
\end{array}
$$

Substituting the coordinates given in Appendix $F$ into Equation I-1, using the small angle approximations $\tan \gamma_{y} \approx \sin \gamma_{y} \approx \gamma_{y}$ and $\cos \gamma_{y} \approx 1$, and recalling that $F_{y}^{I}=F_{y}^{0}$ yields:

$$
Q_{\gamma_{y}}=-M_{A} g L_{c m} \gamma_{y}-F_{z} L \gamma_{y}+2 F_{y}^{0}\left(L-L_{0} / 2\right)
$$

Using Equations 2-10, 2-15, 2-16,2-18 and 2-19 in Equation I-2, and ignoring the $h_{y}^{2}$ term in $F_{z}$ gives:

$$
\begin{aligned}
Q_{y}= & -\left[M_{A} g L_{c m}+\left(6.06 \times 10^{-4}\right) V^{2} L-\left(6.5 \times 10^{-5}\right) V^{2} C\left(z_{L}\right)\left(L-L_{0} / 2\right)^{2}\right] \gamma_{y} \\
& +\left[\left(6.5 \times 10^{-9}\right) V^{2} C\left(z_{L}\right)\left(L-L_{0} / 2\right)\right]\left[10^{4}\left(L_{A} / 2\right) \beta_{y}^{2}+10^{4}\left(\ell-L_{0} / 2\right) \theta_{y}+d_{y}\right]
\end{aligned}
$$

[Q in $\mathrm{N} \cdot \mathrm{cm} ; \mathrm{M}_{A} g$ in $\mathrm{N}$; $V$ in $\mathrm{kV} ; \quad C$ in $p F$; d in $\mu \mathrm{m} ; \mathrm{L}_{\mathrm{cm}}, \mathrm{L}, \mathrm{L}_{0}, \mathrm{~L}_{A}$, $\ell, z_{L}$ in $\mathrm{cm} ; \gamma, \beta, \theta$ in $\left.\mathrm{rad}\right]$. 
b) Derivation of $Q_{B_{y}}$.

Using Equations $3-3$ and 3-4:

$$
\begin{aligned}
Q_{B_{y}}=M_{A} g\left(\partial z_{1} / \partial \beta_{y}\right)+M_{b} g\left(\partial z_{2} / \partial \beta_{y}\right)+M_{B} g\left(\partial z_{3} / \partial \beta_{y}\right) \\
+F_{z}\left(\partial z_{4} / \partial \beta_{y}\right)+F_{y}^{0}\left(\partial y_{5} / \partial \beta_{y}\right)+F_{y}^{I}\left(\partial y_{6} / \partial \beta_{y}\right)
\end{aligned}
$$

Substituting the coordinates given in Appendix F into Equation I-4, realizing that small angle approximations cannot be used, again recalling that $F_{y}^{I}=F_{y}^{0}$, and keeping only the significant terms yields:

$$
\begin{aligned}
Q_{B} & =\left[2 F_{y}^{0} L_{A}-M_{A} g \rho_{P}-M_{b} g \ell_{b}-M_{B} g \ell_{B}-F_{z} \rho_{P}\right] \sin \beta_{y} \\
& +\left[M_{B} g L_{B}-M_{A} g L_{A}-F_{z} L_{A}\right] \cos B_{y} .
\end{aligned}
$$

Using Equations 2-10,2-15, 2-16, 2-18, and 2-19 in Equation I-5, and keeping the $h_{y}^{2}$ part of $F_{z}$ in the $F_{z} L_{A} \cos \beta_{y}$ term of Equation I-5 gives:

$$
\begin{aligned}
Q_{B_{y}}= & {\left[\left(3.25 \times 10^{-5}\right) V^{2} C\left(z_{L}\right) L_{A}^{2} B_{y}^{2}+\left(6.5 \times 10^{-5}\right) V^{2} C\left(z_{L}\right) L_{A}\left(L-L_{o} / 2\right) \gamma_{y}\right.} \\
& +\left(6.5 \times 10^{-5}\right) V^{2} C\left(z_{L}\right) L_{A}\left(\ell-L_{0} / 2\right) \theta y+\left(6.5 \times 10^{-9}\right) V^{2} C\left(z_{L}\right) L_{A} d_{y} \\
& \left.-M_{A} g \rho_{P}-M_{b} g \ell_{b}-M_{B} g \ell_{B}-\left(6.06 \times 10^{-4}\right) V^{2} \rho_{P}\right] \sin \beta_{y} \\
& +\left[M_{B} g L_{B}-M_{A} g L_{A}-\left(6.06 \times 10^{-4}\right) V^{2} L_{A}-\left(3.94 \times 10^{-12}\right) V^{2} L_{A} d_{y}^{2}\right. \\
& -\left(9.85 \times 10^{-5}\right) V^{2} L_{A}^{3} B_{y}^{4}-\left(3.94 \times 10^{-4}\right) V^{2} L_{A}\left(L-L_{0} / 2\right)^{2} \gamma_{y}^{2} \\
& -\left(3.94 \times 10^{-4}\right) V^{2} L_{A}\left(\ell-L_{0} / 2\right)^{2} \theta_{y}^{2}-\left(7.88 \times 10^{-8}\right) V^{2} L_{A}\left(\ell-L_{0} / 2\right) \theta y d \\
& -\left(7.88 \times 10^{-8}\right) V^{2} L_{A}\left(L-L_{0} / 2\right) d_{y} d_{y}-\left(3.94 \times 10^{-8}\right) V^{2} L_{A}^{2} d_{y} B_{y}^{2}
\end{aligned}
$$




$$
\begin{aligned}
& -\left(3.94 \times 10^{-4}\right) V^{2} L_{A}^{2}\left(\ell-L_{0} / 2\right) \theta_{y} \beta_{y}^{2} \\
& -\left(7.88 \times 10^{-4}\right) V^{2} L_{A}\left(L-L_{0} / 2\right)\left(\ell-L_{0} / 2\right) \theta_{y} \gamma_{y} \\
& \left.-\left(3.94 \times 10^{-4}\right) V^{2} L_{A}^{2}\left(L-L_{0} / 2\right) \gamma_{y} \beta_{y}^{2}\right] \cos \beta_{y}
\end{aligned}
$$

[Q in $\mathrm{N} \cdot \mathrm{cm} ; M_{A} g, M_{b} g, M_{B} g$ in $N$; $V$ in $k V ; C$ in $p F ; L_{c m}, L, L_{0}, L_{A}$, $L_{B}, \ell, \ell_{b}, \ell_{B}, \rho_{P}, z_{L}$ in $\mathrm{cm}$; $d$ in $\mu m ; \gamma, B, \theta$ in rad]. 


\section{APPENDIX J}

GENERAL IZED TORQUES FOR $\gamma_{x}$ AND $\beta_{y}$ MOTIONS ONLY

a) Derivation of $Q_{\gamma_{x}}$.

Using Equations 3-3 and 3-4:

$$
\begin{aligned}
Q_{\gamma_{x}}= & M_{A} g\left(\partial z_{1} / \partial \gamma_{x}\right)+M_{b} g\left(\partial z_{2} / \partial \gamma_{x}\right)+M_{B} g\left(\partial z_{3} / \partial \gamma_{x}\right) \\
& +F_{z}\left(\partial z_{4} / \partial \gamma_{x}\right)+F_{x}^{0}\left(\partial x_{5} / \partial \gamma_{x}\right)+F_{x}^{I}\left(\partial x_{6} / \partial \gamma_{x}\right) .
\end{aligned}
$$

Substituting the coordinates given in Appendix G into Equation $\mathrm{J}-1$, using the small angle approximations $\tan \gamma_{X} \approx \sin \gamma_{X} \approx \gamma_{X}$ and $\cos \gamma_{X} \approx$ 1 , and recalling that $F_{X}^{I}=F_{X}^{0}$ yields:

$$
Q_{\gamma_{x}}=-M_{A} g L_{c m} \gamma_{x}-F_{z} L \gamma_{x}+2 F_{x}^{0}\left(L-L_{0} / 2\right)
$$

Using Equations 2-9, 2-14, 2-16, 2-17, and 2-19 in Equation J-2, and ignoring the $h_{x}^{2}$ term in $F_{z}$ gives:

$$
\begin{aligned}
Q_{\gamma_{x}}= & -\left[M_{A} g L_{C m}+\left(6.06 \times 10^{-4}\right) V^{2} L-\left(6.5 \times 10^{-5}\right) V^{2} C\left(z_{L}\right)\left(L-L_{0} / 2\right)^{2}\right] \gamma_{X} \\
& +\left[\left(6.5 \times 10^{-9}\right) V^{2} C\left(z_{L}\right)\left(L-L_{0} / 2\right)\right]\left[10^{4}\left(\ell-L_{0} / 2\right) \theta_{x}+d_{x}\right]
\end{aligned}
$$

[Q in $\mathrm{N} \cdot \mathrm{cm}$; $\mathrm{M}_{A} g$ in $\mathrm{N}$; $V$ in $\mathrm{kV}$; $C$ in $p F ; d$ in $\mu \mathrm{m} ; \mathrm{L}_{\mathrm{cm}}, \mathrm{L}, \mathrm{L}_{0}, \mathrm{~L}_{A}$, $\ell, z_{L}$ in $\mathrm{cm} ; \gamma, \theta$ in $\left.\mathrm{rad}\right]$. 
b) Derivation of $Q_{B_{y}}$.

Using Equations $3-3$ and 3-4:

$$
\begin{aligned}
Q_{B_{y}} & =M_{A} g\left(\partial z_{1} / \partial \beta_{y}\right)+M_{b} g\left(\partial z_{2} / \partial \beta_{y}\right)+M_{B} g\left(\partial z_{3} / \partial \beta_{y}\right) \\
& +F_{z}\left(\partial z_{4} / \partial \beta_{y}\right)+F_{x}^{0}\left(\partial x_{5} / \partial \beta_{y}\right)+F_{x}^{I}\left(\partial x_{6} / \partial \beta_{y}\right) .
\end{aligned}
$$

Substituting the coordinates given in Appendix G into Equation J-4, realizing that small angle approximations cannot be used, and again recalling that $F_{X}^{I}=F_{X}^{0}$, yields:

$$
\begin{aligned}
Q_{B_{y}} & =\left[-M_{A} g \rho_{P}-M_{b} g l_{b}-M_{B} g l_{B}-F_{z^{\rho}} \rho_{P}\right] \sin \beta_{y} \\
& +\left[M_{B} g L_{B}-M_{A} g L_{A}-F_{z} L_{A}\right] \cos \beta_{y^{\circ}}
\end{aligned}
$$

Using Equations 2-9, 2-16 and 2-19 in Equation J-5, and keeping the $h_{x}^{2}$ part of $F_{z}$ in the $F_{z} L_{A} \cos \beta y$ term of Equation $J-5$ gives:

$$
\begin{aligned}
Q_{B_{y}}= & {\left[-M_{A} g \rho_{P}-M_{b} g \ell_{D}-M_{B} g \ell_{B}-\left(6.06 \times 10^{-4}\right) V^{2} \rho_{P}\right] \sin \beta_{y} } \\
& +\left[M_{B} g L_{B}-M_{A} g L_{A} A^{-}\left(6.06 \times 10^{-4}\right) V^{2} L_{A}\right. \\
& -\left(3.94 \times 10^{-4}\right) V^{2}\left(L-L_{0} / 2\right)^{2} L_{A} \gamma_{x}^{2}-\left(3.94 \times 10^{-12}\right) V^{2} L_{A} d_{x}^{2} \\
& -\left(3.94 \times 10^{-4}\right) V^{2}\left(\ell-L_{0} / 2\right)^{2} L_{A} \theta_{x}^{2} \\
& -\left(7.88 \times 10^{-4}\right) V^{2}\left(L-L_{0} / 2\right)\left(\ell-L_{0} / 2\right) L_{A}{ }^{\theta} x^{\gamma} x \\
& -\left(7.88 \times 10^{-8}\right) V^{2}\left(L-L_{0} / 2\right) L_{A} d_{x}{ }^{\gamma} x \\
& \left.-\left(7.88 \times 10^{-8}\right) V^{2}\left(\ell-L_{0} / 2\right) L_{A}{ }^{\theta} d_{x}\right] \cos B_{y}
\end{aligned}
$$

[Q iN $\mathrm{H} \cdot \mathrm{cm} ; M_{A} g, M_{b} g, M_{B} g$ in $N$; $V$ in $k V ; L_{c m}, L, L_{O}, L_{A}, L_{B}, \ell$, $\ell_{b}, \ell_{B}, \rho_{p}$ in $\mathrm{cm} ; d$ in $\mu \mathrm{m} ; \gamma, \theta$ in $\left.\mathrm{rad}\right]$. 


\section{BALANCE BEAM STIFFNESS AND SENSITIVITY}

Let the balance beam and the suspended electrode system be in static equilibrium. Therefore $Q_{B_{y}}=0$, and for very small angles Equation $\mathrm{I}-5$ reduces to

$$
\left[M_{B} g L_{B}-\left(M_{A} g+\hat{F}_{Z}\right) L_{A}\right]=\left[-2 \hat{F}_{y}^{0} L_{A}+\left(M_{A} g+\hat{F}_{z}\right) \rho_{P}+M_{b} g l_{b}+M_{B} g \ell_{B}\right] \hat{\beta}_{y} \text {. }
$$

This equation has the form $\hat{\Gamma}_{B_{y}}=\hat{K \beta_{y}}$, where $\hat{\Gamma}_{B_{y}}$ is the torque in $\mathrm{N} \cdot \mathrm{cm}$, and $K$ is the beam stiffness in $N \cdot \mathrm{cm} / \mathrm{rad}$;

$$
K=\left[-2 \hat{F}_{y}^{0} L_{A}+\left(M_{A} g+\hat{F}_{z}\right) \rho_{P}+M_{b} g l_{b}+M_{B} g l_{B}\right]
$$

[K in $N \cdot \mathrm{cm} / \mathrm{rad} ; \hat{F}_{y}^{0}, \hat{F}_{Z}, M_{A} g, M_{b} g, M_{B} g$ in $N ; L_{A}, \rho_{P}, \ell_{b}, \ell_{B}$ in $\left.\mathrm{cm}\right]$.

The balance beam sensitivity, $S$, is

$$
S=\Delta \hat{\beta}_{y} / \Delta \hat{\Gamma}_{B_{y}}=1 / K
$$

[S in $\mathrm{rad} / \mathrm{N} \cdot \mathrm{cm} ; \hat{B}_{y}$ in $\mathrm{rad} ; \hat{\Gamma}_{B_{y}}$ in $N ; K$ in $\left.N \cdot \mathrm{cm} / \mathrm{rad}\right]$.

We found in part e of Appendix $C$ that the balance beam angle has to be held to within $\pm 5.50 \times 10^{-7} \mathrm{rad}\left( \pm 0.11^{\prime \prime}\right)$ in order to measure the 
capacitance at $50 \mathrm{pF}$ to within $\pm 1 \mathrm{ppm}$. Therefore, in order to monitor $\beta_{y}$, the minimum sensitivity for the addition of a $1 \mu \mathrm{g}$ mass to $M_{A}$ must be

$$
\begin{aligned}
S= & \hat{\beta}_{y} / \Delta \hat{\Gamma}_{B_{y}}=-\left(5.50 \times 10^{-7} \mathrm{rad}\right) /-\left[\left(1 \times 10^{-6} \mathrm{~g}\right)\left(\mathrm{kg} / 10^{3} \mathrm{~g}\right)\left(9.8 \mathrm{~m} / \mathrm{s}^{2}\right)(7.5 \mathrm{~cm})\right]= \\
& 7.48 \mathrm{rad} / \mathrm{N} \cdot \mathrm{cm},
\end{aligned}
$$

or $K=0.134 \mathrm{~N} \cdot \mathrm{cm} / \mathrm{rad}$. With voltage off, $\hat{F}_{z}$ is replaced by $\mathrm{mg}$, and $\hat{F}_{y}^{0}=0$, so Equation $\mathrm{K}-1$ becomes

$$
K^{(O F F)}=\left[\left(M_{A}+m\right) g \rho_{P}+M_{b} g l_{b}+M_{B} g l_{B}\right]
$$

Using the brass electrode, and the values $K^{(\mathrm{OFF})}=0.134 \mathrm{~N} \cdot \mathrm{cm} / \mathrm{rad}$, $M_{A}=5.47 \mathrm{~kg}, m=5 \times 10^{-3} \mathrm{~kg}$, and $\rho_{P}=5 \times 10^{-5} \mathrm{~cm}$, this equation reduces to $18.6 \ell_{b}+53.6 \ell_{B}=0.131$, where $\ell_{b}$ and $\ell_{B}$ are in $\mathrm{cm}$, and can be positive or negative. (They are both drawn with positive signs in Figure 2.)

One has no idea experimentally what the combination $\ell_{b}$ and $\ell_{B}$ actually is, but if $\ell_{b}=0$ then $\ell_{B}=2.44 \times 10^{-3} \mathrm{~cm}(24.4 \mu \mathrm{m})$, or if $\ell_{B}=0$ then $\ell_{b}=7.04 \times 10^{-3} \mathrm{~cm}(70.4 \mu \mathrm{m})$. Clearly, with a reasonable combination of $\ell_{b}$ and $\ell_{B}$, the sensitivity screw on the balance can be adjusted to obtain a value of $S=7.48 \mathrm{rad} / \mathrm{N} \cdot \mathrm{cm}$ for the brass electrode with voltage off.

If $V=9 \mathrm{kV}$ and $\theta_{y}=5$ " then $F_{z}=4.91 \times 10^{-2} \mathrm{~N}$ and $2 F_{y}^{0}=140.7 \times 10^{-5} \mathrm{~N}$ from Table 7 , and Equations $K-1$ and $K-2$ yield the values $K^{(O N)}=0.123 \mathrm{~N} \cdot \mathrm{cm} / \mathrm{rad}$ and $S^{(O N)}=8.13 \mathrm{rad} / \mathrm{N} \cdot \mathrm{cm}$ for $18.6 \ell_{b}+53.6 \ell_{B}=0.131$ 
$N \cdot \mathrm{cm} / \mathrm{rad}$. So $\mathrm{S}^{(\mathrm{ON})}$ depends upon $\hat{F}_{y}^{0}$ (but not upon $\mathrm{F}_{\mathrm{x}}^{0}$ - and is $9 \%$ greater than $S^{(O F F)}$ for this example. This sensitivity variation need not affect us, however, because the voltage can be adjusted so that $\hat{\beta}_{y}^{(O N)}=\hat{\beta}_{y}^{(O F F)}$; i.e. the balance can be used as a null device. (Al though we will see in part $g$ of Appendix $P$ that an error results if $\hat{\beta}_{y}^{(O N)}=\hat{\beta}_{y}^{(O F F)} \neq 0$ and $\hat{F}_{y}^{0} \neq 0$.) 
KINETIC ENERGY FOR $\gamma_{y}$ AND $\beta_{y}$ MOTIONS ONLY

Using Figure 2:

$$
\begin{aligned}
& T=T_{1}+T_{2}+T_{3} \\
& T=(1 / 2) M_{A}\left(\dot{y}_{1}^{2}+\dot{z}_{1}^{2}\right)+(1 / 2) M_{b}\left(\dot{y}_{2}^{2}+\dot{z}_{2}^{2}\right)+(1 / 2) M_{B}\left(\dot{y}_{3}^{2}+\dot{z}_{3}^{2}\right) .
\end{aligned}
$$

Substituting the coordinates listed in Appendix $F$ into the above equation yields:

$$
\begin{aligned}
& T=(1 / 2) M_{A}\left[L_{A}^{2}\left(\dot{\beta}_{y}\right)^{2}+\rho_{p}^{2}\left(\dot{\gamma}_{y}\right)^{2}+L_{C r 0}^{2}\left(\dot{\gamma}_{y}\right)^{2}+\rho_{p}^{2}\left(\dot{B}_{y}\right)^{2}\right. \\
& -2 L_{A} \rho_{p} \sin \beta y \cos \gamma_{y} \dot{B}_{y} \dot{\gamma}_{y} \\
& +2 L_{A} L_{c m} \sin \beta y \cos \gamma_{y} \dot{\beta}_{y} \dot{\gamma}_{y} \\
& +2 \rho_{p}^{2} \cos \beta y \cos \gamma_{y} \dot{\beta}_{y} \dot{\gamma}_{y} \\
& -2 L_{c m}{ }^{\rho} \cos \beta \beta_{y} \cos \gamma_{y} \dot{\beta}_{y} \dot{\gamma}_{y}-2 L_{c m}{ }^{\rho} p\left(\dot{\gamma}_{y}\right)^{2} \\
& -2 L_{A} \rho_{P} \cos \beta{ }_{y} \sin \gamma_{y} \dot{\beta}_{y} \dot{\gamma}_{y} \\
& +2 L_{A} L_{c m} \cos \beta \sin \gamma \dot{\beta}_{y} \dot{\gamma}_{y} \\
& -2 \rho_{p}^{2} \sin \beta y \sin \gamma \dot{\beta}_{y} \dot{\gamma}_{y} \\
& \left.+2 L_{c m} \rho_{p} \sin \beta_{y} \sin \gamma_{y} \dot{\beta}_{y} \dot{\gamma}_{y}\right]+(1 / 2) M_{b} \ell_{b}^{2}\left(\dot{\beta}_{y}\right)^{2} \\
& +(1 / 2) M_{B}\left[L_{B}^{2}\left(\dot{\beta}_{y}\right)^{2}+\ell_{B}^{2}\left(\dot{\beta}_{y}\right)^{2}\right]
\end{aligned}
$$

[T in $\mathrm{kg} \cdot \mathrm{cm}^{2} / \mathrm{s}^{2} ; M_{A}, M_{b}, M_{B}$ in $\mathrm{kg} ; L_{A}, \rho_{P}, L_{c m}, \ell_{b}, L_{B}, \ell_{B}$ in $\mathrm{cm}$; $\dot{\beta}_{y}, \dot{\gamma}_{y}$ in rad/s; $\beta_{y}, \gamma_{y}$ in $\left.\mathrm{rad}\right]$. 
KINETIC ENERGY FOR $\gamma_{x}$ AND $\beta_{y}$ MOTIONS ONLY

Using Figure 2:

$$
\begin{aligned}
& T=T_{1}+T_{2}+T_{3} \\
& T=(1 / 2) M_{A}\left(\dot{x}_{1}^{2}+\dot{z}_{1}^{2}\right)+(1 / 2) M_{b} \dot{z}_{2}^{2}+(1 / 2) M_{B} \dot{z}_{3}^{2} .
\end{aligned}
$$

Substituting the coordinates listed in Appendix $G$ into the above equation yields:

$$
\begin{aligned}
T & =(1 / 2) M_{A}\left[L_{c m}^{2}\left(\dot{\gamma}_{x}\right)^{2}+L_{A}^{2} \cos ^{2} \beta_{y}\left(\dot{\beta}_{y}\right)^{2}+\rho_{P}^{2} \sin ^{2} \beta_{y}\left(\dot{\beta}_{y}\right)^{2}\right. \\
& +2 L_{A} \rho_{P} \sin \beta_{y} \cos \beta_{y}\left(\dot{\beta}_{y}\right)^{2} \\
& +2 L_{c m} L_{A} \sin \gamma_{x} \cos \beta_{y} \dot{\beta}_{y} \dot{\gamma}_{x} \\
& \left.+2 L_{c m^{\rho}} \sin \beta_{y} \sin \dot{\beta}_{x} \dot{\beta}_{y} \dot{\gamma}_{x}\right] \\
& +(1 / 2) M_{b} l_{b}^{2} \sin ^{2} \beta_{y}\left(\dot{\beta}_{y}\right)^{2} \\
& +(1 / 2) M_{B}\left[L_{B}^{2} \cos ^{2} \beta_{y}\left(\dot{\beta}_{y}\right)^{2}-2 L_{B} l_{B} \sin \beta_{y} \cos \beta_{y}\left(\dot{\beta}_{y}\right)^{2}\right. \\
& \left.+e_{B}^{2} \sin ^{2} \beta_{y}\left(\dot{\beta}_{y}\right)^{2}\right]
\end{aligned}
$$

[T in $\mathrm{kg} \cdot \mathrm{cm}^{2} / \mathrm{s}^{2} ; M_{A}, M_{b}, M_{B}$ in $\mathrm{kg} ; L_{A}, L_{c m}, \rho_{P}, \ell_{b}, L_{B}, \ell_{B}$ in $\mathrm{cm}$; $\dot{\beta}_{y}, \dot{\gamma}_{y}$ in rad/s; $\beta_{y}, \gamma_{y}$ in rad]. 
D'ALEMBERT'S PRINCIPLE FOR $\gamma_{y}$ AND $\beta_{y}$ MOTIONS ONLY

a) Equation of motion for the suspended electrode system.

Writing Equation 3-5 so that it has the dimensions $\mathrm{N} \cdot \mathrm{cm}$, yields

$$
10^{-2}\left\{d / d t\left(\partial T / \partial \dot{\gamma}_{y}\right)-\left(\partial T / \partial \gamma_{y}\right)\right\}=Q_{\gamma_{y}} \cdot
$$

Substituting the expressions for $Q_{\gamma_{y}}$ and $T$ given by Equations $I-3$ and $L-1$ into Equation $\mathrm{N}-1$, using the small angle approximations $\sin \gamma_{\mathrm{y}} \approx \gamma_{\mathrm{y}}$, $\cos \gamma_{y} \approx 1, \sin \beta_{y} \approx \beta_{y}$ and $\cos \beta y \approx 1$, keeping only the significant terms, and replacing the point mass $M_{A}$ with a distributed mass, yields the result

$$
\begin{aligned}
& 10^{-2} \mathrm{I}_{\mathrm{P}} \ddot{\gamma}_{\mathrm{y}}+\left[\mathrm{M}_{\mathrm{A}} \mathrm{gL} \mathrm{cm}+\left(6.06 \times 10^{-4}\right) \mathrm{V}^{2} \mathrm{~L}-\left(6.5 \times 10^{-5}\right) \mathrm{V}^{2} \mathrm{C}\left(\mathrm{L}-\mathrm{L}_{0} / 2\right)^{2}\right] \gamma_{\mathrm{y}} \\
& -\left[\left(6.5 \times 10^{-9}\right) V^{2} C\left(L-L_{0} / 2\right)\right]\left[10^{4}\left(\ell-L_{0} / 2\right) \theta_{y}+d_{y}\right]= \\
& -10^{-2} M_{A} L_{c m} L_{A}\left(\dot{\beta}_{y}\right)^{2}-10^{-2} M_{A} L_{c m} L_{A}\left(\beta_{y}+\gamma_{y}\right) \ddot{\beta}_{y}+10^{-2} M_{A} L_{c m} \rho_{p} \ddot{\beta}_{y} \\
& +\left[\left(6.5 \times 10^{-9}\right) V^{2} C\left(L-L_{0} / 2\right) 10^{4}\left(L_{A} / 2\right)\right] \beta_{y}^{2}
\end{aligned}
$$

$\left[I_{P}\right.$ in $\mathrm{kg} \cdot \mathrm{cm}^{2} ; M_{A}$ in $\mathrm{kg} ; g$ in $\mathrm{m} / \mathrm{s}^{2} ; \mathrm{L}_{\mathrm{cm}}, \mathrm{L}, \mathrm{L}_{0}, \ell, \mathrm{L}_{A}, \rho_{P}$ in $\mathrm{cm}$; $V$ in $\mathrm{kV} ; C$ in $\mathrm{pF} ; d_{y}$ in $\mu \mathrm{m} ; \gamma_{y}, \beta_{y}, \theta_{y}$ in $\left.\mathrm{rad}\right]$. 
where $I_{P}$ is the moment of inertia of the suspended electrode system about the pivot point $P$ of Figure 2, which from the parallel axis theorem is equal to the moment of inertia about the center of mass of the suspension system plus the mass times distance squared from the center of mass to the pivot point: $I_{P}=\left(I_{S}\right)_{c m}+M_{A} L_{c m}^{2}$. Using the brass cylinder in the suspension system gives $I_{p} \approx 1.39 \times 10^{4} \mathrm{~kg} \cdot \mathrm{cm}^{2}$, which is $15 \%$ larger than the value of $M_{A} L_{c m}^{2}$. The off-diagonal, product of inertia terms are represented by the point mass expression $M_{A} L_{c m} L_{A}$ in Equation $\mathrm{N}-2$; this is a very good approximation. The $10^{-2} M_{A} L_{c m} L_{A}\left(\dot{\beta}_{y}\right)^{2}$ and $10^{-2} M_{A} L_{c m} L_{A}\left(\beta_{y}+\gamma_{y}\right) \ddot{\beta_{y}}$ terms are the torques induced about point $P$ due to the respective centripetal and tangential accelerations of the suspended electrode system resulting from the motion of the balance beam about point $C$ of Figure 2. Equation $\mathrm{N}-2$ has the form:

$$
a \ddot{\gamma}_{y}+b \gamma_{y}-c=-e\left(\dot{\beta}_{y}\right)^{2}-e\left(\beta_{y}+\gamma_{y}\right) \ddot{\beta} y+\ddot{\beta_{y}}+k \beta_{y}^{2}
$$

where the coefficients are defined in Equation $\mathrm{N}-2$.

b) Equation of motion for the balance beam.

Writing Equation 3-5 so that it again has the dimensions $\mathrm{N} \cdot \mathrm{cm}$, yields

$$
10^{-2}\left\{d / d t\left(\partial T / \partial \dot{\beta}_{y}\right)-\left(\partial T / \partial \beta_{y}\right)\right\}=Q_{\beta_{y}} \cdot
$$


It is clear from inspection of Equation I- 6 that it would not be possible to find an analytical solution of Equation I-4 without simplifying the terms involving $\beta_{\mathrm{y}}$. We want to detect $1 \mathrm{\mu g}$ in 5.47 $\mathrm{kg}$; therefore the balance must have a resolution of at least 1.8 parts in $10^{10}$. If we approximate $\cos \beta_{y}=1$ by ignoring the $-\beta_{y}^{2} / 2$ term, then $\beta_{y}$ must be less than or equal to $1.94 \times 10^{-5} \mathrm{rad}\left(4^{\prime \prime}\right)$ in order to keep the errors at the 1.8 parts in $10^{10}$ level. So, let the balance swing freely with an amplitude and equilibrium angle such that $\beta_{y} \leq 4 "$. We can then obtain an analytical solution; this solution should also be valid for larger values of $\beta_{y}$, except, possibly, for amplitude-dependent errors, which could be corrected by extrapolating the balance measurement values to a zero-amplitude result.

Substituting the expressions for $Q_{B_{y}}$ and $T$ given by Equations I- 6 and $\mathrm{L}-1$ into Equation $\mathrm{N}-4$, using the small angle approximations $\sin \beta_{y} \approx \beta_{y}, \cos \beta_{y} \approx 1, \sin \gamma_{y} \approx \gamma_{y}$ and $\cos \gamma_{y}{ }^{* 1}$, keeping only the significant terms, and replacing the point masses with distributed masses, yields the result

$$
\begin{aligned}
& 10^{-2} I_{C} \ddot{\beta}_{y}+\left\{M_{A} g \rho_{P}+M_{b} g \ell_{b}+M_{B} g \ell_{B}+\left(6.06 \times 10^{-4}\right) V^{2} \rho_{P}\right. \\
& \left.-\left(6.5 \times 10^{-5}\right) V^{2} C L_{A}\left[\left(\ell-L_{0} / 2\right) \theta_{y}+10^{-4} d_{y}\right]\right\}_{B} \\
& -\left[\left(6.5 \times 10^{-5}\right) V^{2} C_{A}\left(L-L_{0} / 2\right)\right] \gamma_{y} \beta_{y} \\
& -\left\{M_{B} g L_{B}-M_{A} g L_{A}-\left(6.06 \times 10^{-4}\right) V^{2} L_{A}\right. \\
& \left.-\left(3.94 \times 10^{-8}\right) V^{2} L_{A}\left[10^{-4} d_{y}^{2}+10^{4}\left(\ell-L_{0} / 2\right)^{2} \theta_{y}^{2}+2\left(\ell-L_{0} / 2\right) \theta_{y} d_{y}\right]\right\} \\
& =-10^{-2} M_{A} L_{A} L_{c m}\left(\dot{\gamma}_{y}\right)^{2}-10^{-2} M_{A} L_{A} L_{c m}\left(\beta_{y}+\gamma_{y}\right) \ddot{\gamma}_{y} \\
& +10^{-2} M_{A} L_{c m} \rho_{P} \ddot{\gamma}_{y}-\left[\left(3.94 \times 10^{-4}\right) V^{2} L_{A}\left(L-L_{0} / 2\right)^{2}\right] \gamma_{y}^{2}
\end{aligned}
$$




$$
-\left\{\left(7.88 \times 10^{-4}\right) V^{2} L_{A}\left(L-L_{0} / 2\right)\left[\left(\ell-L_{0} / 2\right) \theta_{y}+10^{-4} d_{y}\right]\right\} \gamma_{y}
$$

$\left[I_{C}\right.$ in $\mathrm{kg} \cdot \mathrm{cm}^{2} ; M_{A}, M_{b}, M_{B}$ in $\mathrm{kg} ; g$ in $\mathrm{m} / \mathrm{s}^{2} ; L_{A}, l_{b}, l_{B}, \rho_{P}, l, L$, $L_{0}, L_{c m}, L_{B}$ in $\mathrm{cm}$; $V$ in $\mathrm{kV}$; $C$ in $\mathrm{pF}$; $d_{y}$ in $\mu \mathrm{m} ; \gamma_{y}, \beta_{y}, \theta_{y}$ in $\mathrm{rad}]$.

where the moment of inertia, $I_{C}$, about point $C$ of Figure 2 is $I_{C}=M_{A} L_{A}^{2}+\left(I_{C W}\right)_{B}+M_{B} L_{B}^{2}+\left(I_{b}\right)_{C}$, and $\left(I_{C W}\right)_{B}$ and $\left(I_{b}\right)_{C}$ are the moments of inertia of the counterweight about point $B$ and of the balance beam about point $C$, respectively. Using the brass cylinder $\left(M_{A}=M_{B}=5.47\right.$ kg) gives $I_{C} \approx 683 \mathrm{~kg} \cdot \mathrm{cm}^{2}$. The $10^{-2} M_{A} L_{A} L_{c m}\left(\dot{\gamma}_{y}\right)^{2}$ and $10^{-2} M_{A} L_{A} L_{c m}\left(\beta_{y}+\gamma_{y}\right) \ddot{\gamma}_{y}$ terms are torques induced about point $C$ due to the respective centripetal and tangential accelerations of the suspended electrode system about point $P$ of Figure 2.

Equation $\mathrm{N}-5$ has the form:

$$
\ddot{n} \ddot{\beta}_{y}+\left(0-p \gamma_{y}\right) \beta_{y}-q=-e\left(\dot{\gamma}_{y}\right)^{2}-e\left(\beta_{y}+\gamma_{y}\right) \ddot{\gamma} \ddot{\gamma}_{y}+f \ddot{\gamma}_{y}-r \gamma_{y}^{2}-s \gamma_{y}
$$

where the coefficients are defined in Equations $\mathrm{N}-2, \mathrm{~N}-3$ and $\mathrm{N}-5$. 
D'ALEMBERT'S PRINCIPLE FOR $\gamma_{x}$ AND $\beta_{y}$ MOTIONS ONLY

a) Equation of motion for the suspended electrode system.

Writing Equation $3-5$ so that it has the dimensions $N \cdot \mathrm{cm}$, yields

$$
10^{-2}\left\{d / d t\left(\partial T / \partial \dot{\gamma}_{x}\right)-\left(\partial T / \partial \gamma_{x}\right)\right\}=Q_{\gamma_{x}}
$$

Substituting the expressions for $Q_{\gamma_{x}}$ and $T$ given by Equations $\mathrm{J}-3$ and M-1 into Equation 0-1, using the small angle approximations $\sin \gamma_{x} \approx \gamma_{X}$, $\cos \gamma x \approx 1, \sin \beta \beta^{\approx} \beta_{y}$ and $\cos \beta y \approx 1$, keeping only the significant terms, and replacing the point mass $M_{A}$ with a distributed mass, yields the result

$$
\begin{aligned}
10^{-2} I_{P} \ddot{\gamma}_{x}+\left[M_{A} g L_{c m}+\left(6.06 \times 10^{-4}\right) V^{2} L-\left(6.5 \times 10^{-5}\right) V^{2} C\left(L-L_{0} / 2\right)^{2}\right] \gamma_{x} \\
-\left[\left(6.5 \times 10^{-9}\right) V^{2} C\left(L-L_{0} / 2\right)\right]\left[10^{4}\left(\ell-L_{0} / 2\right) \theta_{x}+d_{x}\right] \\
=-10^{-2} M_{A} L_{c m} L_{A} \gamma_{x} \ddot{\beta}_{y}
\end{aligned}
$$

$\left[I_{P}\right.$ in $\mathrm{kg} \cdot \mathrm{cm}^{2} ; M_{A}$ in $\mathrm{kg} ; g$ in $\mathrm{m} / \mathrm{s}^{2} ; \quad V$ in $\mathrm{kV} ; L_{c m}, L, L_{0}, \ell, L_{A}$ in $\mathrm{cm}$; $C$ in $\mathrm{pF} ; d_{x}$ in $\mu \mathrm{m} ; \gamma_{x}, \theta_{x}, \beta_{y}$ in $\left.\mathrm{rad}\right]$

where $I_{P}$ is defined in Appendix $N$, and is $1.39 \times 10^{4} \mathrm{~kg} \cdot \mathrm{cm}^{2}$ for the brass cylinder. The $10^{-2} M_{A} L_{c m} L^{\gamma} x_{x} \ddot{\beta}_{y}$ term is the torque induced about 
point $P$ of Figure 2 due to the tangential acceleration of the suspended electrode system resulting from the balance beam motion about point $C$. The centripetal acceleration term is negligible. Equation $0-2$ has the form:

$$
a \ddot{\gamma}_{x}+b \gamma_{x}-c^{\prime}=-e \gamma_{x} \ddot{\beta}_{y}
$$

where the coefficients are defined in Equations $0-2, \mathrm{~N}-2$, and $\mathrm{N}-3$.

b) Equation of motion for the balance beam.

Writing Equation 3-5 so that it again has the dimensions $\mathrm{N} \cdot \mathrm{cm}$, yields

$$
10^{-2}\left\{d / d t\left(\partial T / \partial \dot{\beta}_{y}\right)-\left(\partial T / \partial \beta_{y}\right)\right\}=Q_{\beta_{y}} \cdot
$$

We assume, for the reasons given in Appendix $N$, that $\beta_{y} \leq 1.94 \times 10^{-5}$ $\operatorname{rad}\left(4^{\prime \prime}\right)$.

Substituting the expressions for $Q_{B_{y}}$ and $T$ given by Equations J-6 and $M-1$ into Equation 0-4, using the small angle approximations $\sin \beta_{y} \approx \beta_{y}, \cos \beta_{y} \approx 1, \sin \gamma_{x} \approx \gamma_{x}$ and $\cos \gamma_{x} \approx 1$, keeping only the significant terms, and replacing the point masses with distributed masses, yields the result

$$
10^{-2} I_{C} \ddot{\beta}_{y}+\left[M_{A} g \rho_{p}+M_{b} g \ell_{b}+M_{B} g \ell_{B}+\left(6.06 \times 10^{-4}\right) V^{2} \rho_{p}\right] \beta_{y}
$$




$$
\begin{aligned}
& -\left\{M_{B} g L_{B}-M_{A} g L_{A}-\left(6.06 \times 10^{-4}\right) V^{2} L_{A}\right. \\
& -\left(3.94 \times 10^{-8}\right) V^{2} L_{A}\left[10^{-4} d_{x}^{2}\right. \\
& \left.\left.\quad+10^{4}\left(\ell-L_{0} / 2\right)^{2} \theta_{x}^{2}+2\left(\ell-L_{0} / 2\right) \theta_{x} d_{x}\right]\right\} \\
& =-10^{-2} M_{A} L_{c m} L_{A}\left(\dot{\gamma}_{x}\right)^{2}-10^{-2} M_{A} L_{c m} L_{A} \gamma_{x} \ddot{\gamma}_{x} \\
& \quad-\left(3.94 \times 10^{-4}\right) V^{2}\left(L-L_{0} / 2\right)^{2} L_{A} \gamma_{x}^{2} \\
& -\left\{\left(7.88 \times 10^{-4}\right) V^{2}\left(L-L_{0} / 2\right) L_{A}\left[\left(\ell-L_{0} / 2\right) \theta_{x}+10^{-4} d_{x}\right]\right\} \gamma_{x}
\end{aligned}
$$

$\left[I_{C}=k g \cdot \mathrm{cm}^{2} ; M_{A}, M_{b}, M_{B}\right.$ in $\mathrm{kg} ; g$ in $\mathrm{m} / \mathrm{s}^{2} ; L_{A}, \ell_{b}, \ell_{B}, \rho_{P}, \ell, L, L_{0}$, $L_{c m}, L_{B}$ in $\mathrm{cm}$; $V$ in $\mathrm{kV}$; $C$ in $\mathrm{pF}$; $d_{x}$ in $\mu \mathrm{m} ; \gamma_{x}, B_{y}, \theta_{x}$ in rad]

where $I_{C}$ is defined in Appendix $N$, and is $683 \mathrm{~kg} \cdot \mathrm{cm}^{2}$ for the brass cylinder. The $10^{-2} M_{A} L_{c m} L_{A}\left(\dot{\gamma}_{x}\right)^{2}$ and $10^{-2} M_{A} L_{c m} L_{A} \gamma_{x} \ddot{\gamma}_{x}$ terms are torques induced about point $C$ due to the respective centripetal and tangential accelerations of the suspended electrode system about point $P$ of Figure 2.

Equation $0-5$ has the form:

$$
\ddot{n} \ddot{\beta}_{y}+v \beta_{y}-q^{\prime}=-e\left(\dot{\gamma}_{x}\right)^{2}-e \gamma_{x} \ddot{\gamma}_{x}-r \gamma_{x}^{2}-s^{\prime} \gamma_{x}
$$

where the coefficients are defined in Equations $0-2,0-3,0-5, \mathrm{~N}-2$, $\mathrm{N}-3$ and $\mathrm{N}-5$. 


\section{APPENDIX P}

SOLUTIONS OF THE EQUATIONS OF MOTION FOR $\gamma_{y}$ AND $\beta_{y}$ OSCILLATIONS ONLY

a) The suspended electrode motion.

We found in Equation $\mathrm{N}-3$ of Appendix $\mathrm{N}$ that

$$
\begin{aligned}
& \ddot{\gamma}_{y}+(b / a) \gamma_{y}-(c / a)=-(e / a)\left(\dot{\beta}_{y}\right)^{2} \\
& \quad-(e / a)\left(\beta_{y}+\gamma_{y}\right) \ddot{\beta}_{y}+(f / a) \ddot{\beta}_{y}+(k / a)\left(\beta_{y}{ }^{2}\right)
\end{aligned}
$$

where the coefficients are defined in Equation $\mathrm{N}-2$. The homogenous equation $\ddot{\gamma}_{y}+(b / a) \gamma_{y}-(c / a)=0$ has a periodic solution, which depends upon the starting conditions. If $\gamma_{y}$ happens to be at its miminum value at time $t=0$, then

$$
\gamma_{y}=-\tilde{\gamma}_{y} \cos \omega_{\gamma_{y}} t+\hat{\gamma}_{y}
$$

where $\tilde{\gamma}_{y}$ is the amplitude of the oscillation and $\hat{\gamma}_{y}$ is the equilibrium angle.

We will find in part e that the approximate solution of the $\beta_{y}$ equation is also periodic - and, if $\beta_{y}$ is at its mimimum value at $t=0$, then

$$
\beta_{y}=-\tilde{\beta}_{y} \cos \omega_{\beta_{y}} t+\hat{\beta}_{y} \text {. }
$$


Equations $P-2$ and $P-3$ are approximate solutions for the $\gamma_{y}$ and $\beta_{y}$ motions. We next treat the r.h.s. of Equation $\mathrm{P}-1$ as a perturbation of the 1.h.s. of the equation [11], with $\beta_{y}$ having the form of Equation $P-3$. Taking the appropriate time derivatives of $\beta_{y}$, and noting that $\cos ^{2} \alpha-\sin ^{2} \alpha=\cos 2 \alpha$ and that $\cos ^{2} \alpha=(1 / 2)(1+\cos 2 \alpha)$ yields the result

$$
\begin{aligned}
& \ddot{\gamma}_{y}+\left[(b / a)+(e / a) \tilde{\beta}_{y} \omega_{\beta_{y}}^{2} \cos \omega_{\beta_{y}} t\right] \gamma_{y}-\left[(c / a)+(k / a)\left(\hat{\beta}_{y}^{2}+\tilde{\beta}_{y}^{2} / 2\right)\right] \\
& =\left[(e / a) \tilde{\beta}_{y}^{2} \omega_{\beta_{y}}^{2}+(k / 2 a) \tilde{\beta}_{y}^{2}\right] \cos 2 \omega_{\beta_{y}}{ }^{t} \\
& +\left[(f / a) \tilde{\beta}_{y} \omega_{\beta_{y}}^{2}-(e / a) \tilde{\beta}_{y} \hat{\beta} \omega_{\beta_{y}}^{2}-(2 k / a) \tilde{\beta}_{y} \hat{\beta}_{y}\right] \cos \omega_{\beta_{y}} t
\end{aligned}
$$

The homogenous equation for the 1.h.s. of Equation P-4 has the form of Mathieu's equation $[12,13]$ unless $b>e_{\tilde{\beta}} \tilde{y}_{\beta_{y}}^{2}$. Using values given in Table 8 for $V=9 \mathrm{kV}$ and $\tilde{\beta}_{y}=2.40 \times 10^{-3} \mathrm{rad}$ (rather than $1.94 \times 10^{-5} \mathrm{rad}$ ), yields $689 \mathrm{~N} \cdot \mathrm{cm} \gg 0.001 \mathrm{~N} \cdot \mathrm{cm}$; so, the $(e / a) \tilde{\beta}_{y} \omega_{B_{y}}^{2} \cos \omega_{\beta_{y}} t$ term can be ignored in Equation $P-4$, and $\gamma_{y}$ is primarily a cosine function rather than a Mathieu function.

The perturbation solution of Equation P-4 is then exact and, if $\omega_{\gamma_{y}} \neq \omega_{\beta_{y}}$ or $\omega_{\gamma_{y}} \neq 2 \omega_{\beta_{y}}$, the solution is [13]:

$$
\begin{aligned}
\gamma_{y}=-\tilde{\gamma}_{y} \cos \omega_{\gamma_{y}} t+\hat{\gamma}_{y} \\
+\left[(e / a) \tilde{\beta}_{y}^{2(1)} \beta_{y}^{2}+(k / 2 a) \tilde{\beta}_{y}^{2}\right] \cos 2 \omega_{\beta_{y}} t /\left(\omega_{\gamma_{y}}^{2}-4()_{\beta_{y}}^{2}\right)
\end{aligned}
$$




$$
+\left[(f / a) \tilde{\beta}_{y} \omega_{B_{y}}^{2}-(e / a) \tilde{\beta}_{y} \hat{\beta}_{y} \omega_{B_{y}}^{2}-(2 k / a) \tilde{\beta}_{y} \hat{\beta}_{y}\right] \cos \omega_{\beta_{y}} t /\left(\omega_{\gamma_{y}}^{2}-\omega_{\beta_{y}}^{2}\right) .
$$

Therefore, the suspended electrode system executes simple harmonic motion about the equilibrium angle $\hat{\gamma}_{y}$ with $\cos 2 \omega_{\beta_{y}}{ }^{t}$ and $\cos \omega_{\beta_{y}} t$ amplitude modulations.

b) The equilibrium angle $\hat{\gamma}_{y}$.

It follows from Equations $P-4$ and $P-5$ that

$$
\begin{aligned}
& \hat{\gamma}_{y}=\left[c+k\left(\hat{\beta}_{y}^{2}+(1 / 2) \tilde{\beta}_{y}^{2}\right)\right] / b \\
& \hat{\gamma}_{y}=\left(6.5 \times 10^{-9}\right) V^{2} C\left(L-L_{0} / 2\right)\left[10^{4}\left(\ell-L_{0} / 2\right) \theta_{y}+d_{y}+10^{4}\left(L_{A} / 2\right)\left(\hat{\beta}_{y}^{2}+\tilde{\beta}_{y}^{2} / 2\right)\right] / \\
& \quad\left[M_{A} g L_{c m}+\left(6.06 \times 10^{-4}\right) v^{2} L-\left(6.5 \times 10^{-5}\right) v^{2} c\left(L-L_{0} / 2\right)^{2}\right]
\end{aligned}
$$

[V in kV; $C$ in pF; $M_{A} g$ in $N ; L, L_{0}, \ell, L_{A}, L_{c m}$ in $c m ; d_{y}$ in $\mu m$; $\hat{\gamma}_{y}, \theta_{y}, \hat{\beta}_{y}, \tilde{\beta}_{y}$ in rad].

So, $\hat{\gamma}_{y}$ depends upon $d_{y}, \theta_{y}, \hat{\beta}_{y}$ and $\tilde{\beta}_{y}$ for a given $V, C$ and $L_{0}$. Using values from Table 8 in Equation P-6 results in a change in $\hat{\gamma}_{y}$ of $0.1^{\prime \prime}$ when $\tilde{\beta}_{y}=2.40 \times 10^{-3} \mathrm{rad}$ and $\hat{\beta}_{y} \approx 0$. Therefore, $\hat{\gamma}_{y}$ is not affected very much by the balance beam motion. 
c) The period $\mathrm{P}_{\gamma_{y}}$.

It follows from Equations $P-4$ and $P-5$ that:

$$
\omega_{\gamma_{y}}^{2}=b / a=\left[M_{A} g L_{c m}+\left(6.06 \times 10^{-4}\right) V^{2} L-\left(6.5 \times 10^{-5}\right) V^{2} C\left(L-L_{0} / 2\right)^{2}\right] /\left[10^{-2} I_{P}\right]
$$

$\left[\omega_{\gamma_{y}}\right.$ in $\mathrm{rad} / \mathrm{s} ; M_{A} g$ in $\mathrm{N} ; \mathrm{L}_{\mathrm{cm}}, \mathrm{L}, \mathrm{L}_{0}$ in $\mathrm{cm}$; $V$ in $\mathrm{kV}$; $C$ in $p F ; I_{P}$ in $\mathrm{kg} \cdot \mathrm{cm}^{2}$,

and

$$
P_{\gamma_{y}}=2 \pi / \omega_{\gamma_{y}}
$$

$\left[P_{\gamma_{y}}\right.$ in $s ; \omega_{\gamma_{y}}$ in $\left.\mathrm{rad} / \mathrm{s}\right]$

$P_{\gamma_{y}}$ and $\omega_{\gamma_{y}}$ are plotted versus $V$ in Figure 10 using values from Table 8 in Equation P-7. The points shown in this figure are at the values of $V$ which provide electrical forces that are equivalent to gravitational forces due to masses of $0,1,2,3,4$ and 5 grams. $P_{\gamma_{y}}$ increases from a value of $1.48 \mathrm{~s}$ at $V=0 \mathrm{kV}$ to a value of $2.82 \mathrm{~s}$ at $V=9 \mathrm{kV}$. If the voltage were increased to the point of unstable equilibrium (10.56 kV for this example) then $\mathrm{P}_{\gamma_{y}}{ }^{+\infty}$, and the suspended electrode would collide with the carriage electrode in a time interval which depends upon the initial position of the suspended electrode and its horizontal velocity component. 
d) The amplitude modulations of $\gamma_{y}$.

The amplitude of the $\cos 2 \omega_{\beta_{y}}{ }^{t}$ modulation term in Equation P-5 is

$$
\begin{aligned}
& {\left[10^{-2} M_{A} L_{c m} L_{A} \omega_{B_{y}}^{2}+\left(3.25 \times 10^{-9}\right) V^{2} c\left(L-L_{0} / 2\right) 10^{4}\left(L_{A} / 2\right)\right] \tilde{B}_{y}^{2} /} \\
& {\left[10^{-2} I_{p}\left(\omega_{\gamma_{y}}^{2}-4 \omega_{\beta_{y}}^{2}\right)\right]}
\end{aligned}
$$

$\left[M_{A}\right.$ in $\mathrm{kg} ; \mathrm{I}_{P}$ in $\mathrm{kg} \cdot \mathrm{cm}^{2} ; \mathrm{L}_{\mathrm{cm}}, \mathrm{L}_{A}, \mathrm{~L}, \mathrm{~L}_{0}$ in $\mathrm{cm} ; \mathrm{V}$ in $\mathrm{kV} ; C$ in $\mathrm{pF}$; $\omega_{\gamma_{y}}, \omega_{\beta_{y}}$ in rad/s; $\tilde{\beta}_{y}$ in rad],

and using values from Table 8 and $\tilde{\beta}_{y}=2.40 \times 10^{-3}$ rad, has the value 7.7 $\times 10^{-7} \mathrm{rad}\left(0.16^{\prime \prime}\right)$. So, the $\gamma_{y}$ cosine waveform of $2.82 \mathrm{~s}$ period would have a \pm 0.16 " modulation imposed upon it, with a period $P_{B_{y}}=46.8 \mathrm{~s}$ for this example. A sufficiently sensitive autocollimator that monitored the angle of a mirror mounted on top of the suspended electrode could detect this amplitude modulation of $\gamma_{y}$.

The amplitude (for sufficiently small $\hat{\beta}_{y}$ ) of the $\cos \omega_{\beta_{y}} t$ modulation term in Equation P-5 is

$$
\left[M_{A} L_{c m}{ }^{\rho} p \omega_{B_{y}}^{2} \tilde{\beta}_{y}\right] /\left[I_{p}\left(\omega_{\gamma_{y}}^{2}-\omega_{\beta_{y}}^{2}\right)\right]
$$

$\left[M_{A}\right.$ in $\mathrm{kg} ; \mathrm{I}_{P}$ in $\mathrm{kg} \cdot \mathrm{cm}^{2} ; \mathrm{L}_{\mathrm{cm}}, \rho_{P}$ in $\mathrm{cm} ; \omega_{\gamma}, \omega_{\beta_{y}}$ in $\mathrm{rad} / \mathrm{s} ; \tilde{\beta}_{y}$ in $\mathrm{rad}]$,

and has a value $4.1 \times 10^{-6}$ " for the values from Table 8 . So, the $\cos \omega_{\beta_{y}} t$ modulation would not be detectable. 
e) The balance beam motion.

We found in Equation $N-6$ of Appendix $N$ that

$$
\begin{aligned}
\ddot{\beta}_{y} & {\left[(0 / n)-(p / n) \gamma_{y}\right] \beta_{y}-(q / n)=-(e / n)\left(\dot{\gamma}_{y}\right)^{2}-(e / n)\left(\beta_{y}+\gamma_{y}\right) \ddot{\gamma}_{y} } \\
& +(f / n) \ddot{\gamma}_{y}-(r / n) \gamma_{y}^{2}-(s / n) \gamma_{y}
\end{aligned}
$$

where the coefficients are defined in Equations $\mathrm{N}-2, \mathrm{~N}-3$ and $\mathrm{N}-5$.

The r.h.s. of Equation P-11 can be treated as a perturbation of the 1.h.s. of the equation. Using the approximate solution for $\gamma_{y}$ given hy Equation $P-2$, taking the appropriate time derivatives of $\gamma_{y}$, and noting that $\cos ^{2} \alpha-\sin ^{2} \alpha=\cos 2 \alpha$ and that $\cos ^{2} \alpha=(1 / 2)(1+\cos 2 \alpha)$ yields the result

$$
\begin{gathered}
\ddot{\beta}_{y}+\left[(1 / n)\left(0-p \hat{\gamma}_{y}\right)+\left(\tilde{\gamma}_{y} / n\right)\left(p+e \omega_{\gamma_{y}}^{2}\right) \cos \omega_{\gamma_{y}} t\right] \beta_{y} \\
-\left[(1 / n)\left(q-r \hat{\gamma}_{y}^{2}-s \hat{\gamma}_{y}-(r / 2) \tilde{\gamma}_{y}^{2}\right)\right] \\
=\left[\left(\tilde{\gamma}_{y}^{2} / n\right)\left(e \omega_{\gamma_{y}}^{2}-r / 2\right)\right] \cos 2 \omega_{\gamma_{y}} t \\
+\left[\left(\tilde{\gamma}_{y} / n\right)\left(f \omega_{\gamma_{y}}^{2}+2 \hat{\gamma}_{y}+s-\hat{\gamma}_{y} \omega_{\gamma_{y}}^{2}\right)\right] \cos \omega_{\gamma_{y}} t .
\end{gathered}
$$

The homogenous equation for the 1.h.s. of Equation P-4 has the form of Mathieu's equation $[12,13]$ unless $\left(0-p \hat{\gamma}_{y}\right)=\kappa^{(0 N)}>\tilde{r}_{y}\left(p+e \omega_{\gamma_{y}}^{2}\right)$. Using values given in Table 8 , and letting $\tilde{\gamma}_{y}=\hat{\gamma}_{y}=6.8^{\prime \prime}$, results in the 
values $0.123 \mathrm{~N} \cdot \mathrm{cm} / \mathrm{rad}>0.011 \mathrm{~N} \cdot \mathrm{cm} / \mathrm{rad}$. So, if $\tilde{\gamma}_{y}<6.8^{\prime \prime}$, the $(1 / n)\left(p+e \omega_{\gamma_{y}}^{2}\right) \tilde{\gamma}_{y} \cos \omega_{\gamma_{y}} t$ term can be ignored in Equation $P-12$, and $\beta_{y}$ is primarily a cosine function rather than a Mathieu function.

The perturbation solution of Equation P-12 is then exact - and if $\beta_{y}$ happens to be at its minimum value at time $t=0$, and if $\omega_{\beta_{y}} \neq \omega_{\gamma_{y}}$ or $\omega_{\beta_{y}} \neq 2 \omega_{\gamma_{y}}$, the solution is [13]:

$$
\begin{aligned}
\beta_{y}= & -\tilde{\beta}_{y} \cos \omega_{\beta_{y}} t+\hat{\beta}_{y} \\
& +\left[\left(\tilde{\gamma}_{y} / n\right)^{2}\left(e \omega_{\gamma_{y}}^{2}-r / 2\right)\right] \cos 2 \omega_{\gamma_{y}} t /\left(\omega_{\beta_{y}}^{2}-4 \omega_{\gamma_{y}}^{2}\right) \\
& +\left[\left(\tilde{\gamma}_{y} / n\right)\left(f \omega_{\gamma_{y}}^{2}+2 r \hat{\gamma}_{y}+s-e \hat{\gamma}_{y} \omega_{\gamma_{y}}^{2}\right)\right] \cos \omega_{\gamma_{y}} t /\left(\omega_{\beta_{y}}^{2}-\omega_{\gamma_{y}}^{2}\right) .
\end{aligned}
$$

Therefore, the balance beam executes simple harmonic motion about the equilibrium angle $\hat{\beta}_{y}$ with $\cos 2 \omega_{\gamma_{y}} t$ and $\cos \omega_{\gamma_{y}} t$ amplitude modulations.

f) The equilibrium angle $\hat{\beta}_{y}$.

It follows from Equations $P-12$ and $P-13$ that

$$
\begin{aligned}
\hat{\beta}_{y}= & {\left[q-r \hat{\gamma}_{y}^{2}-s \hat{\gamma}_{y}-(r / 2) \tilde{\gamma}_{y}^{2}\right] /\left(0-p \hat{\gamma}_{y}\right)=} \\
& {\left[q-r \hat{\gamma}_{y}^{2}-s \hat{\gamma}_{y}-(r / 2) \tilde{\gamma}_{y}^{2}\right] / K } \\
\hat{B}_{y}= & \left\{M_{B} g L_{B}-M_{A} g L_{A}-\left(6.06 \times 10^{-4}\right) v^{2} L_{A}\right. \\
& -\left(3.94 \times 10^{-8}\right) v^{2} L_{A}\left[10^{-4} d_{y}^{2}+10^{4}\left(l-L_{0} / 2\right)^{2} \theta_{y}^{2}+2\left(l-L_{0} / 2\right) \theta_{y} d_{y}\right] \\
& -\left(3.94 \times 10^{-4}\right) v^{2} L_{A}\left(L-L_{0} / 2\right)^{2}\left[\hat{\gamma}_{y}^{2}+(1 / 2) \tilde{\gamma}_{y}^{2}\right]
\end{aligned}
$$




$$
\begin{aligned}
& \left.-\left(7.88 \times 10^{-4}\right) V^{2} L_{A}\left(L-L_{o} / 2\right)\left[\left(\ell-L_{0} / 2\right) \theta_{y}+10^{-4} d_{y}\right] \hat{\gamma}_{y}\right\} / \\
& \left\{M_{A} g \rho_{P}+M_{b} g \ell_{b}+M_{B} g \ell_{B}+\left(6.06 \times 10^{-4}\right) V^{2} \rho_{P}\right. \\
& -\left(6.5 \times 10^{-5}\right) V^{2} C L_{A}\left[\left(\ell-L_{0} / 2\right) \theta_{y}+10^{-4} d_{y}\right] \\
& \left.-\left(6.5 \times 10^{-5}\right) V^{2} C L_{A}\left(L-L_{o} / 2\right) \hat{\gamma}_{y}\right\}
\end{aligned}
$$

$\left[M_{A} g, M_{b} g, M_{B} g\right.$ in $N$; $V$ in $k V ; C$ in $p F ; L_{A}, L_{B}, L, \ell, L_{0}, \rho p, \ell_{b}$, $\ell_{B}$ in $\mathrm{cm} ; d_{y}$ in $\mu \mathrm{m} ; \hat{\beta}_{y}, \theta_{y}, \hat{\gamma}_{y}, \tilde{\gamma}_{y}$ in $\left.\mathrm{rad}\right]$.

So, $\hat{\beta}_{y}$ depends upon $\theta_{y}, d_{y}, \hat{\gamma}_{y}$ and $\tilde{\gamma}_{y}$ for a given $V, C$ and $L_{0}$. The variation in $\hat{\beta}_{y}$ due to $\tilde{\gamma}_{y}$ is

$$
\delta \hat{B}_{y}=-\left(1.97 \times 10^{-4}\right) V^{2} L_{A}\left(L-L_{0} / 2\right)^{2} \tilde{\gamma}_{y}^{2} / K
$$

[V in $\mathrm{kV} ; \mathrm{L}_{A}, \mathrm{~L}, \mathrm{~L}_{0}$ in $\mathrm{cm} ; \quad K$ in $N \cdot \mathrm{cm} / \mathrm{rad} ; \hat{\beta}_{y}, \tilde{\gamma}_{y}$ in $\left.\mathrm{rad}\right]$.

where $K$ is defined by Equations $K-1,2-10,2-15,2-16,2-18$ and 2-19. Using values from Table 8 in Equation $P-15$ gives, for $V=9 k V$ :

$$
\delta \hat{\beta}_{y}^{(0 N)}=-\left(1.64 \times 10^{-2}\right) \tilde{\gamma}_{y}^{2}
$$

$$
\left[\hat{\beta}_{y}, \tilde{\gamma}_{y}\right. \text { in "] }
$$

where $\hat{\beta}_{y}^{(O N)}$ and $\tilde{\gamma}_{y}$ are now expressed in arcseconds in order to get a feeling for the magnitude of this effect. $\delta \hat{\beta}_{y}^{(O N)}$ is plotted versus $\tilde{\gamma}_{y}$ in Figure 11 for $y=9 \mathrm{kV}$. If $\tilde{\gamma}_{y}=9$ ", then $\hat{\beta}_{y}^{(O N)}$ is $1.33^{\prime \prime}$ less 
than its value for $\tilde{\gamma}_{y}=0$; so, $F_{z}$ would appear to be larger by an amount equivalent to a $12 \mu \mathrm{g}$ mass increase because the balance would have been calibrated using sensitivity weights with the voltage off yielding a $\Delta \hat{\beta}_{y}^{(O F F)} / \Delta m$ calibration value of $0.11 \% / \mu$.

The value of $\tilde{\gamma}_{y}$ depends upon the starting condition. If, in this example, the suspended electrode was released, at rest, from gravitational vertical $\left(\gamma_{y}=0\right)$, then $\tilde{\gamma}_{y}=\hat{\gamma}_{y}=6.8^{\prime \prime}$, making $F_{z}$ appear to be larger by an amount equivalent to a $7 \mu \mathrm{g}$ mass increase. Figure 11 shows that $\tilde{\gamma}_{y}$ has to be less than $2.5^{\prime \prime}$ in order to measure the mass equivalent of $F_{z}$ to within $1 \mu \mathrm{g}$ at $V=9 \mathrm{kV}$. Note, from Equation $P-15$, that the systematic error, $\delta \hat{\beta}_{y}$, is zero for the voltage-off measurements.

The amplitude, $\tilde{\gamma}_{y}$, of the suspended electrode will decrease with time; if only velocity-dependent damping occurs, then the $\gamma_{y}$ waveform given by Equation P-5 would have an exponential decay envelope superimposed upon it - and, since the velocities are small, the decay time-constant would be long. The actual rate at which $\tilde{\gamma}_{y}$ decreases is not yet known, but it could be monitored by autocollimator measurements via a mirror mounted on top of the suspended electrode.

g) The force determination.

The voltage will be adjusted in the force measurements until 


$$
\hat{\beta}_{y}^{(O N)}=\hat{\beta}_{y}^{(O F F)} \text {. }
$$

Using Equations $P-14,2-10,2-15,2-16,2-18,2-19$ and $K-1$, and realizing that $\hat{F}_{z}=\hat{F}_{y}^{0}=0$ and that $M_{A} \rightarrow M_{A}+m$ for $V=0$, Equation $P-17$ becomes, when neglecting the $\left(6.06 \times 10^{-4}\right) V^{2} \rho_{p}$ term:

$$
\begin{aligned}
& {\left[M_{B} g L_{B}-M_{A} g L_{A}-\hat{F}_{Z} L_{A}-\left(1.97 \times 10^{-4}\right) V^{2} L_{A}\left(L-L_{0} / 2\right)^{2} \tilde{\gamma}_{y}^{2}\right] /} \\
& \quad\left[K(0 F F)-2 F_{y} L_{A}\right] \\
& =\left[M_{B} g L_{B}-\left(M_{A}+m\right) g L_{A}\right] / K^{(0 F F),}
\end{aligned}
$$

which can be rewritten in the form:

$$
\hat{F}_{z}=m g\left[1+\left(2 \hat{F}_{y}^{0} / m g\right) \hat{\beta}_{y}^{(0 F F)}-(1 / m g)\left(1.97 \times 10^{-4}\right) v^{2}\left(L-L_{0} / 2\right)^{2} \tilde{\gamma}_{y}^{2}\right] \quad(P-18)
$$

$\left[\hat{F}_{z}, \hat{F}_{y}^{0}, m g\right.$ in $N$; $V$ in $k V ; L, L_{0}$ in $\mathrm{cm} ; \hat{\beta}_{y}, \tilde{\gamma}_{y}$ in $\left.\mathrm{rad}\right]$.

Therefore, $\hat{F}_{z}=m g$ only if $\hat{F}_{y}^{0}=0$ or $\hat{B}_{y}^{(O F F)}=0$, and if $\tilde{\gamma}_{y}=0$.

The dynamical error term $-(1 / \mathrm{mg})\left(1.97 \times 10^{-4}\right) V^{2}\left(L_{-} L_{0} / 2\right)^{2} \tilde{\gamma}_{y}^{2}$ leads to a systematic error in $\hat{F}_{z}$ of $-1.2 \mathrm{ppm}$ at $V=9 \mathrm{kV}$ and $C=100 \mathrm{pF}$ for $\tilde{\gamma}_{y}=6.8^{\prime \prime}$, and a systematic error of $-0.2 \mathrm{ppm}$ for $\tilde{\gamma}_{y}=2.5^{\prime \prime}$; i.e. the voltages were lower than they should have been if $\hat{F}_{z}$ were to be equal to $\mathrm{mg}$. This error term can be experimentally monitored by measuring $\tilde{\gamma}_{y}$ with the suspended electrode autocollimator. It always has a negative sign. 
Values for the static error term $(1 / \mathrm{mg}) 2 \hat{F}_{y}^{0} \hat{\beta}_{y}(\mathrm{OFF})$ are listed in Tables 3-7 in ppm/", where the "symbol refers to the $\hat{\beta}_{y}^{(O F F)}$ angle in arcseconds. If $\hat{\beta}_{y}^{(O F F)}=-10$ " with the brass suspended electrode at $V=9 \mathrm{kV}, d_{y}=0$ and $\theta_{y}=+5 "$, then Table 7 provides the resulting systematic error in $\hat{F}_{z}$ of $-1.4 \mathrm{ppm}$.

The angle $\hat{\beta}_{y}^{(\text {OFF })}$ is measured from gravitational horizon. So, a method will have to be devised to determine, with sufficient accuracy, the balance beam readout angle that corresponds to the plane defined by the contact points $(P)$ and $(C)$ of the pivot and center knives (see Figures 2-4) being parallel with gravitational horizon. In the above example the horizontal plane determination would have to be better than 7" in order for the static error term to be less than $1 \mathrm{ppm}$.

Either the counterweight mass, $M_{B}$, or $M_{A}$ will then have to be adjusted until the correct readout angle is obtained with voltage-off. For a balance mass sensitivity of $0.11 \% / \mu \mathrm{g}$, this means that the $M_{B}$ or $M_{A}$ adjustment must be to within 7" (or $64 \mu \mathrm{g}$ ) for a combined possible error in $\hat{\mathrm{F}}_{\mathrm{z}}$ of $2 \mathrm{ppm}$. More than the usual care must then be taken after adjustment, and the readout angle mirror mounts must be mechanically stable.

The sign of $\hat{F}_{y}^{0}$ can be determined by monitoring $\hat{r}_{y}$ with the suspended electrode autocollimator, but the sign of $\hat{\beta}_{y}^{(O F F)}$ could only be determined if gravitational horizon were accurately known. Therefore, the sign of the systematic static error term is unknown. 
Neither error term occurs for normal mass intercomparisons. The dynamic term is zero because $V=0$, and the static term is zero because $\hat{F}_{y}^{0}=0$, independent of the value of $\hat{\beta}_{y}^{(O F F)}$.

h) The period $P_{B_{y}}$.

It follows from Equations $P-12$ and $P-13$ that

$$
\begin{aligned}
& \omega_{B_{y}}^{2}=(1 / n)\left(0-p \hat{\gamma}_{y}\right)=K / n . \\
& \omega_{\beta_{y}^{2}}^{2}=\left[1 /\left(10^{-2} I_{C}\right)\right]\left[M_{A} g \rho_{P}+M_{b} g l_{b}+M_{B} g l_{B}+\left(6.06 \times 10^{-4}\right) V^{2} \rho_{P}\right. \\
& -\left(6.5 \times 10^{-5}\right) V^{2} C_{A}\left[\left(\ell-L_{0} / 2\right) \theta_{y}+10^{-4} d_{y}+\left(L-L_{0} / 2\right) \hat{\gamma}_{y}\right]
\end{aligned}
$$

$\left[\omega_{B_{y}}\right.$ in rad/s; $M_{A} g, M_{b} g, M_{B} g$ in $N$; $V$ in $k V ; C$ in $P F ; \rho_{p}, \ell_{b}, l_{B}$, $L_{A}, L, \ell, L_{0}$ in $\mathrm{cm}$; $d_{y}$ in $\mu \mathrm{m} ; \theta_{y}, \hat{\gamma}_{y}$ in $\mathrm{rad} ; I_{C}$ in $\left.\mathrm{kg} \cdot \mathrm{cm}^{2}\right]$,

and

$$
P_{B_{y}}=2 \pi / \omega_{B_{y}}
$$

$\left[P_{B_{y}}\right.$ in s; $\omega_{B_{y}}$ in rad/s $]$.

$P_{\beta_{y}}$ depends upon $\theta_{y}, d_{y}$ and $\hat{\gamma}_{y}$ for a given $V, C$ and $L_{o}$. Using values from Table 8 in Equations $P-19$ and $P-20, P_{B_{y}}$ increases from a value of $44.9 \mathrm{~s}$ at $V=0 \mathrm{kV}$ to a value of $46.8 \mathrm{~s}$ at $V=9 \mathrm{kV}$. 
i) The amplitude modulations of $\beta_{y}$.

The amplitude of the $\cos ^{2} \omega_{\gamma_{y}} t$ modulation term in Equation P-13 is

$$
\begin{gathered}
\tilde{\gamma}_{y}^{2}\left[10^{-2} M_{A} L_{A} L_{C m} \omega_{\gamma_{y}}^{2}-\left(1.97 \times 10^{-4}\right) V^{2} L_{A}\left(L-L_{0} / 2\right)^{2}\right] / \\
{\left[10^{-2} I_{C}\left(\omega_{B_{y}}^{2}-4 \omega_{\gamma_{y}}^{2}\right)\right]}
\end{gathered}
$$

$\left[M_{A}\right.$ in $\mathrm{kg} ;{ }_{\sim}{ }_{C}$ in $\mathrm{kg} \cdot \mathrm{cm}^{2} ; \mathrm{L}_{\mathrm{Cm}}, \mathrm{L}_{A}, \mathrm{~L}, \mathrm{~L}_{0}$ in $\mathrm{cm} ; V$ in $\mathrm{kV} ; \omega_{B_{y}}, \omega_{\gamma_{y}}$ in $\mathrm{rad} / \mathrm{s} ; \tilde{\gamma}_{y}$ in $\left.\mathrm{rad}\right]$.

Using values from Table 8 in Equation P-21, and assuming that $\tilde{\gamma}_{y}=\hat{\gamma}_{y}=3.30 \times 10^{-5} \mathrm{rad}\left(6.8^{\prime \prime}\right)$ at $V=9 \mathrm{kV}$; then the amplitude is $2.6 \times 10^{-9} \mathrm{rad}\left(0.00054^{\prime \prime}\right)$. So, the $\beta_{y}$ cosine waveform of $46.8 \mathrm{~s}$ period would have a $\pm 0.00054 "$ modulation imposed upon it, with a period $P_{\gamma_{y}}=2.82 \mathrm{~s}$ for this example. The balance would not be sensitive enough to detect this modulation.

The amplitude of the $\cos \omega_{\gamma_{y}} t$ term in Equation $P-5$ is

$$
\begin{aligned}
\tilde{\gamma}_{y} /\left[10^{-2} I_{C}\left(\omega_{B}^{2}-\omega_{\gamma_{y}}^{2}\right)\right]\left[10^{-2} M_{A} L_{c m} \rho_{P} \omega_{\gamma_{y}}^{2}-10^{-2} M_{A} L_{A} L_{c m} \hat{\gamma}_{y} \omega_{\gamma_{y}}^{2}\right. \\
\left.+(7.88 \times 10) v^{2} L_{A}\left(L-L_{0} / 2\right)\left[\left(\ell-L_{0} / 2\right) \theta y+\left(L-L_{0} / 2\right) \hat{\gamma}_{y}+10^{-4} d_{y}\right]\right\}
\end{aligned}
$$

$\left[M_{A}\right.$ in $\mathrm{kg} ; I_{C}$ in $\mathrm{kg} \cdot \mathrm{cm}^{2} ; \mathrm{L}_{\mathrm{Cm}}, \rho_{P}, \mathrm{~L}_{A}, \mathrm{~L}, \mathrm{~L}_{0}, \ell$ in $\mathrm{cm} ; \quad V$ in $\mathrm{kV}$; $\omega_{B_{y}}, \omega_{\gamma_{y}}$ in rad/s; $d_{y}$ in $\mu \mathrm{m} ; \tilde{\gamma}_{y}, \hat{\gamma}_{y}, \theta_{y}$ in $\left.\mathrm{rad}\right]$. 
Using the above example, the amplitude is $-7.2 \times 10^{-8} \mathrm{rad}\left(-0.015^{\prime \prime}\right)$, which is a factor of 7 smaller than the $0.11 \% / \mu g$ mass sensitivity of the balance, and is therefore undetectable. 


\section{APPENDIX $Q$}

SOLUTIONS OF THE EQUATIONS OF MOTION FOR $\gamma_{x}$ and $\beta_{y}$ OSCILLATIONS ONLY.

a) The suspended electrode motion.

We found in Equation 0-3 of Appendix 0 that

$$
\ddot{\gamma}_{x}+(b / a) \gamma_{x}-\left(c^{\prime} / a\right)=-(e / a) \gamma_{x} \ddot{\beta}_{y}
$$

where the coefficients are defined in Equations $\mathrm{N}-2, \mathrm{~N}-3$ and $0-2$. The homogenous equation $\ddot{\gamma}_{x}+(b / a) \gamma_{x}-\left(c^{\prime} / a\right)=0$ has a periodic solution, which depends upon the starting conditions. If $\gamma_{x}$ is at its minimum value at time $t=0$, then

$$
\gamma_{x}=-\tilde{\gamma}_{x} \cos \omega_{\gamma_{x}} t+\hat{\gamma}_{x}
$$

where $\tilde{\gamma}_{x}$ is the amplitude of the oscillation and $\hat{\gamma}_{x}$ is the equilibrium angle.

We found in part e of Appendix $P$ that the approximate solution of the $\beta_{y}$ equation is also periodic - and, if $\beta_{y}$ is at its minimum value at $t=0$, then

$$
\beta_{y}=-\tilde{\beta}_{y} \cos \omega_{B_{y}} t+\hat{\beta}_{y}
$$


Equations Q-2 and Q-3 are approximate solutions for the $\gamma_{x}$ and $\beta_{y}$ motions. We next treat the r.h.s. of Equation $Q-1$ as a perturbation of the 1.h.s. of the equation [11], with $\beta_{y}$ having the form of Equation P-3. The result is

$$
\ddot{\gamma}_{x}+\left[(b / a)+(e / a) \omega_{\beta_{y}}^{2} \cos \omega_{\beta_{y}} t\right] \gamma_{x}-\left(c^{\prime} / a\right)=0
$$

This has the form of Mathieu's equation $[12,13]$, but we found in part a of Appendix $P$ that $b>>\tilde{\beta}_{y} \omega_{\beta_{y}}^{2}$, so

$$
\ddot{\gamma}_{x}+(b / a) \gamma_{x}-\left(c^{\prime} / a\right)=0
$$

The solution of Equation Q-5 is

$$
\gamma_{x}=-\tilde{\gamma}_{x} \cos \omega_{\gamma_{x}} t+\hat{\gamma}_{x}
$$

Therefore the suspended electrode system executes simple harmonic motion about the equilibrium angle $\hat{\gamma}_{x}$.

b) The equilibrium angle $\hat{\gamma}_{x}$.

It follows from Equations $Q-5$ and $Q-6$ that

$$
\begin{aligned}
\hat{\gamma_{x}}=c^{\prime} / b= & \left(6.5 \times 10^{-9}\right) V^{2} c\left(L-L_{0} / 2\right)\left[10^{4}\left(\ell-L_{0} / 2\right) \theta x+d_{x}\right] / \\
& {\left[M_{A} g L_{c m}+\left(6.06 \times 10^{-4}\right) V^{2} L-\left(6.5 \times 10^{-5}\right) V^{2} C\left(L-L_{0} / 2\right)^{2}\right] }
\end{aligned}
$$


[V in $\mathrm{kV} ; C$ in $\mathrm{pF} ; \mathrm{M}_{\mathrm{A}} \mathrm{g}$ in $\mathrm{N} ; \mathrm{L}, \mathrm{L}_{0}, \ell, \mathrm{L}_{\mathrm{cm}}$ in $\mathrm{Cm} ; \mathrm{d}_{\mathrm{x}}$ in $\mu \mathrm{m} ; \hat{\gamma}_{\mathrm{X}}$, $\theta_{x}$ in $\left.\mathrm{rad}\right]$.

So, $\hat{\gamma}_{x}$ depends upon $\theta_{x}$ and $d_{x}$ for a given $V, C$ and $L_{0}$.

c) The period $P_{\gamma_{X}}$.

It follows from Equations $Q-5$ and $Q-6$ that

$$
\omega_{\gamma_{x}}^{2}=b / a=\omega_{\gamma_{y}}^{2}=\left[M_{A} g L_{C m}+\left(6.06 \times 10^{-4}\right) V^{2} L-\left(6.5 \times 10^{-5}\right) V^{2} C\left(L-L_{0} / 2\right)^{2}\right] /\left[10^{-2} I_{P}\right]
$$

$\left[\omega_{\gamma_{x}}\right.$ in rad/s; $M_{A} g$ in $N$; $L_{c m}, L, L_{0}$ in $\mathrm{cm} ; \quad V$ in $k V ; C$ in $p F ; I_{P}$ in $\left.\mathrm{kg} \cdot \mathrm{cm}^{2}\right]$, and

$$
\begin{gathered}
P_{\gamma_{x}}=P_{\gamma_{y}}=2 \pi / \omega_{\gamma_{x}} \\
{\left[P_{\gamma_{x}}, P_{\gamma_{y}} \text { in s; } \omega_{\gamma_{x}} \text { in } \mathrm{rad} / \mathrm{s}\right] .}
\end{gathered}
$$

So, $P_{\gamma_{x}}=P_{\gamma_{y}}$, and for the case given by Table $8, P_{\gamma_{X}}$ increases from a value of $1.48 \mathrm{~s}$ at $V=0 \mathrm{kV}$ to a value of $2.82 \mathrm{~s}$ at $V=9 \mathrm{kV}$.

d) The balance beam motion.

We found in Equation 0-6 of Appendix 0 that 


$$
\ddot{\beta}_{y}+(v / n) \beta_{y}-\left(q^{\prime} / n\right)=-(e / n)\left(\dot{\gamma}_{x}\right)^{2}-(e / n) \gamma_{x} \ddot{\gamma}_{x}-(r / n) \gamma_{x}^{2}-\left(s^{\prime} / n\right) \gamma_{x}
$$

where the coefficients are defined in Equations $\mathrm{N}-2, \mathrm{~N}-3, \mathrm{~N}-5,0-2$, $0-3$ and $0-5$. The r.h.s. of Equation Q-10 can be treated as a perturbation of the I.h.s. of the equation. Using the solution for $\gamma_{x}$ given by Equation Q-6, taking the appropriate time derivatives of $\gamma_{x}$, and noting that $\cos ^{2} \alpha-\sin ^{2} \alpha=\cos 2 \alpha$ and that $\cos ^{2} \alpha=(1 / 2)(1+\cos 2 \alpha)$ yields the result

$$
\begin{aligned}
& \ddot{\beta}_{y}+(v / n) \beta_{y}-\left[(1 / n)\left(q^{\prime}-r \hat{\gamma}_{x}^{2}-s^{\prime} \hat{\gamma}_{x}-(r / 2) \tilde{\gamma}_{x}^{2}\right]\right. \\
& =\left[\left(\tilde{\gamma}_{x}^{2} / n\right)\left(e \omega_{\gamma_{x}}^{2}-r / 2\right)\right] \cos 2 \omega_{\gamma_{x}} t \\
& +\left[\left(\tilde{\gamma}_{x} / n\right)\left(2 r \hat{\gamma}_{x}-e \hat{\gamma}_{x} \omega_{\gamma_{x}}^{2}+s^{\prime}\right)\right] \cos \omega_{\gamma_{x}} t .
\end{aligned}
$$

The perturbation solution of Equation Q-11 is then exact - and if $\beta_{y}$ is at its minimum value at time $t=0$, and if $\omega_{\beta_{y}}^{\prime} \neq \omega_{\gamma_{x}}$ or $\omega_{\beta_{y}}^{\prime} \neq 2 \omega_{\gamma_{x}}$, the solution is [13]:

$$
\begin{aligned}
\beta_{y}= & -\tilde{\beta}_{y} \cos \omega_{\beta_{y}}^{t} t+\hat{\beta}_{y}^{\prime} \\
& +\left[\left(\tilde{\gamma}_{x}^{2} / n\right)\left(e \omega_{\gamma_{x}}^{2}-r / 2\right)\right] \cos 2 \omega_{\gamma_{x}} t /\left(\omega_{\beta_{y}^{\prime}}^{2}-4 \omega_{\gamma_{x}}^{2}\right) \\
& +\left[\left(\tilde{\gamma}_{x} / n\right)\left(2 r \hat{\gamma}_{x}-e \hat{\gamma}_{x} \omega_{\gamma_{x}}^{2}+s^{\prime}\right)\right] \cos \omega_{\gamma_{x}} t /\left(\omega_{\beta_{y}^{\prime}}^{2}-\omega_{\gamma_{x}}^{2}\right) .
\end{aligned}
$$


Therefore, the balance beam executes simple harmonic motion about the equilibrium angle $\hat{\beta}_{y}^{\prime}$ with $\cos 2 \omega_{\gamma_{x}} t$ and $\cos \omega_{\gamma_{x}} t$ amplitude modulations, where $\omega_{\gamma_{x}}=\omega_{\gamma_{y}}$.

e) The equilibrium angle $\hat{\beta}_{y}^{\prime}$.

It follows from Equations $Q-11$ and $Q-12$ that

$$
\begin{aligned}
\hat{\beta}_{y}^{\prime}= & {\left[q^{\prime}-r_{x}^{2}-s^{\prime} \hat{\gamma}_{x}-(r / 2) \tilde{\gamma}_{x}^{2}\right] / v=\left[q^{\prime}-r \hat{\gamma}_{x}^{2}-s^{\prime} \hat{\gamma}_{x}-(r / 2) \tilde{\gamma}_{x}^{2}\right] / } \\
& {\left[K^{(0 F F)}+\left(6.06 \times 10^{-4}\right) v^{2} \rho_{P}\right] } \\
\hat{\beta}_{y}^{\prime}= & \left\{M_{B} g L_{B}-M_{A} g L_{A}-\left(6.06 \times 10^{-4}\right) v^{2} L_{A}\right. \\
& -\left(3.94 \times 10^{-8}\right) v^{2} L_{A}\left[10^{-4} d_{x}^{2}+10^{4}\left(\ell-L_{o} / 2\right)^{2} \theta_{x}^{2}+2\left(\ell-L_{o} / 2\right) \theta_{x} d_{x}\right] \\
& -\left(3.94 \times 10^{-4}\right) v^{2}\left(L-L_{o} / 2\right)^{2} L_{A}\left[\gamma_{x}^{2}+\tilde{\gamma}_{x}^{2} / 2\right] \\
& \left.-\left(7.88 \times 10^{-4}\right) v^{2}\left(L-L_{o} / 2\right) L_{A}\left[\left(\ell-L_{o} / 2\right) \theta_{x}+10^{-4} d_{x}\right] \hat{\gamma}_{x}\right\} / \\
& \left\{M_{A} g \rho_{P}+M_{b} g l_{b}+M_{B} g l_{B}+\left(6.06 \times 10^{-4}\right) v^{2} \rho_{P}\right\}
\end{aligned}
$$

$\left[M_{A} g, M_{b} g, M_{B} g\right.$ in $N$; $V$ in $k V ; C$ in $p F ; L_{A}, L_{B}, L, \ell, L_{0}, \rho_{p}, \ell_{b}$, $\ell_{B}$ in $\mathrm{cm} ; d_{x}$ in $\mu \mathrm{m} ; \hat{\beta}_{y}^{\prime}, \theta_{x}, \hat{\gamma}_{x}, \tilde{\gamma}_{x}$ in $\left.\mathrm{rad}\right]$.

So, $\hat{\beta}_{y}^{\prime}$ depends upon $\theta_{x}, d_{x}, \hat{\gamma}_{x}$ and $\tilde{\gamma}_{x}$ for a given $V, C$ and $L_{0}$.

Equations $P-14$ and Q-13 imply that when $\gamma_{x}, \gamma_{y}$ and $\beta_{y}$ motions all occur that there would be a single value of $\hat{\beta}_{y}$ which depended upon $\theta_{x}, \theta_{y}, d_{x}, d_{y}, \hat{\gamma}_{x}, \hat{\gamma}_{y}, \tilde{\gamma}_{x}$ and $\tilde{\gamma}_{y}$ for a given $v, C$ and $L_{0}$. 
The variation in $\hat{\beta}_{y}^{\prime}$ due to $\tilde{\gamma}_{x}$ is

$$
\begin{aligned}
\delta \hat{\beta}_{y}^{\prime}= & -\left[\left(1.97 \times 10^{-4}\right) V^{2} L_{A}\left(L-L_{0} / 2\right)^{2} \tilde{\gamma}_{x}^{2}\right] / \\
& {\left[K^{(0 F F)}+\left(6.06 \times 10^{-4}\right) V^{2} \rho_{P}\right] }
\end{aligned}
$$

$\left[V\right.$ in $k V ; L_{A}, L, L_{0}, \rho_{P}$ in $\mathrm{cm} ; \quad K$ in $N \cdot \mathrm{cm} / \mathrm{rad} ; \hat{\beta}_{y}^{\prime}, \tilde{\gamma}_{x}$ in rad]

where $X^{(O F F)}$ is defined by Equations $K-1,2-9,2-14,2-16,2-17$ and 2-19. Using values from Table 8 in Equation $Q-14$ gives, for $V=9 k V$ :

$$
\delta \hat{\beta}_{y}^{\prime(O N)}=-\left(1.51 \times 10^{-2}\right) \tilde{\gamma}_{x}^{2}
$$

$\left[\hat{\beta}_{y}^{\prime}, \tilde{\gamma}_{x}\right.$ in " $]$.

This is slightly different from Equation $P-16$ for $\gamma_{y}, B_{y}$ motion because the balance beam stiffness is different in the two cases.

f) The force determination.

The voltage is adjusted in the force measurements until

$$
\hat{\beta}_{y}^{\prime(O N)}=\hat{\beta}_{y}^{\prime}(O F F) .
$$


Using Equations Q-13, 2-9,2-14, 2-16, 2-17, 2-19 and $K-1$, arid realizing that $\hat{F}_{Z}=0$ and that $M_{A} \rightarrow M_{A}+m$ for $V=0$, Equation $Q-16$ becomes, when neglecting the $\left(6.06 \times 10^{-4}\right) V^{2} \rho_{p}$ term:

$$
\begin{aligned}
& {\left[M_{B} g L_{B}-M_{A} g L_{A}-\hat{F}_{Z} L_{A}-\left(1.97 \times 10^{-4}\right) V^{2} L_{A}\left(L-L_{0} / 2\right)^{2} \tilde{\gamma}_{X}^{2}\right] / K^{(O F F)}} \\
& \quad=\left[M_{B} g L_{B}-\left(M_{A}+m\right) g L_{A}\right] / K^{(0 F F)} \\
& \hat{F}_{z}=m g\left[1-(1 / \mathrm{mg})\left(1.97 \times 10^{-4}\right) V^{2}\left(L-L_{0} / 2\right)^{2} \tilde{\gamma}_{X}^{2}\right]
\end{aligned}
$$

$\left[\hat{\mathrm{F}}, \mathrm{mg}\right.$ in $\mathrm{N} ; \quad \mathrm{V}$ in $\mathrm{kV} ; \mathrm{L}, \mathrm{L}_{0}$ in $\mathrm{cm} ; \tilde{\gamma}_{\mathrm{x}}$ in $\left.\mathrm{rad}\right]$.

Equations $P-18$ and Q-17 imply that when $\gamma_{x}, \gamma_{y}$ and $B_{y}$ motions all occur that the static error term for $\hat{F}_{z}$ depends only upon $\hat{F}_{y}^{0}$ and not upon $\hat{F}_{X}^{0}$, but that the dynamical error term depends upon both $\tilde{\gamma}_{X}^{2}$ and $\tilde{\gamma}_{y}^{2}$

g) The period $P_{B_{y}}^{\prime}$.

It follows from Equations $Q-11$ and $Q-12$ that

$$
\begin{aligned}
& w_{B_{y}}^{2}=v / n=\left[K^{(0 F F)}+\left(6.06 \times 10^{-4}\right) v^{2} \rho_{P}\right] / n \\
& \omega_{B_{y}}^{2}=\left[1 /\left(10^{-2} I_{C}\right)\right]\left\{M_{A} g \rho_{P}+M_{b} g l_{b}+M_{B} g l_{B}+\left(6.06 \times 10^{-4}\right) v^{2} \rho_{P}\right\}
\end{aligned}
$$


$\left[\omega_{B_{y}}^{\prime}\right.$ in rad/s; $M_{A} g, M_{B} g, M_{b} g$ in $N ; V$ in $k V ; \quad \ell_{b}, \ell_{B}, \rho_{P}$ in cml $\mathrm{I}_{C}$ in $\left.\mathrm{kg} \cdot \mathrm{cm}^{2}\right]$,

and

$$
P_{\beta_{y}}^{\prime}=2 \pi / \omega_{\beta_{y}}^{\prime}
$$

$\left[P_{\beta_{y}}^{\prime}\right.$ in $s ; \omega_{\beta_{y}}^{1}$ in $\left.\mathrm{rad} / \mathrm{s}\right]$

For the case given in Table $8, P_{\beta_{y}}(O N)=P_{\beta_{y}}(O F F)=P_{\beta_{y}}(O F F)=44.9 \mathrm{~s}$, and $\omega_{\beta_{y}}^{(O N)}=\omega_{\beta_{y}^{\prime}}^{(O F F)}=\omega_{\beta_{y}}(\mathrm{OFF})=0.140 \mathrm{rad} / \mathrm{s}$. A comparison of Equations $P-19$ and Q-18 implies that when $\gamma_{x}, \gamma_{y}$ and $\beta_{y}$ motions all occur that Equation $P-19$ would represent the correct expression for $\omega_{\beta_{y}}$ because Equation $Q-18$ is independent of $\theta_{x}, d_{x}$ and $\hat{\gamma}_{x}$.

Note that the solutions to the equations of motion do not predict large energy transfers between the $B_{y}$ motion and the $\gamma_{x}$ and $\gamma_{y}$ motions for the sample calculations. Therefore, $\tilde{\gamma}_{x}, \tilde{\gamma}_{y}$ and $\tilde{\beta}_{y}$ are not time dependent (except for damping effects). This is because $\omega_{\gamma_{x}}$ and $\omega_{\gamma_{y}}$ are very much greater than $\omega_{B_{y}}$. If the angular frequencies were nearly equal, then there would be large amplitude modulations on the $\gamma_{y}$ and $\beta_{y}$ waveforms as predicted by Equations $P-5$, $\mathrm{P}-13$ and $\mathrm{Q}-12$. 
h) The amplitude modulations of $\beta_{y}$.

The amplitude of the $\cos 2 \omega_{\gamma_{X}} t$ modulation term in Equation 0-12, remembering that $\omega_{\gamma_{x}}=\omega_{\gamma_{y}}$, is

$$
\begin{aligned}
& \tilde{\gamma}_{x}^{2}\left[10^{-2} M_{A} L_{C i n} L_{A} \omega_{\gamma_{y}^{2}}^{2}-\left(1.97 \times 10^{-4}\right) V^{2} L_{A}\left(L-L_{0} / 2\right)^{2}\right] / \\
& {\left[10^{-2} I_{C}\left(\omega_{B_{y}}^{2}-4 \omega_{\gamma_{y}}^{2}\right)\right]}
\end{aligned}
$$

$\left[M_{A}\right.$ in $\mathrm{kg} ; I_{C}$ in $\mathrm{kg} \cdot \mathrm{cm}^{2} ; L_{c m}, L_{A}, L, L_{0}$ in $\mathrm{cm} ; V$ in $k V ; \omega_{\beta_{y}}, \omega_{\gamma_{y}}$ in $\mathrm{rad} / \mathrm{s} ; \tilde{\gamma}_{x}$ in $\left.\mathrm{rad}\right]$.

The example given in part $i$ of Appendix $P$ shows that this amplitude modulation of the $\beta_{y}$ cosine waveform would be undetectable.

The amplitude of the $\cos \omega_{\gamma_{x}} t$ modulation term in Equation Q-12, again remembering that $\omega_{\gamma_{x}}=\omega_{\gamma_{y}}$, is

$$
\begin{aligned}
\tilde{\gamma}_{x} /\left[10^{-2} I_{C}\left(\omega_{B_{y}}^{2}{ }^{-\omega_{\gamma}^{2}}\right)\right]\left\{-10^{-2} M_{A} L_{A} L_{c m} \hat{\gamma}_{x} \omega_{\gamma_{y}}^{2}\right. \\
\left.+\left(7.88 \times 10^{-4}\right) V^{2} L_{A}\left(L-L_{0} / 2\right)\left[\left(\ell-L_{0} / 2\right) \theta_{x}+\left(L-L_{0} / 2\right) \hat{\gamma}_{x}+10^{-4} d_{x}\right]\right\}
\end{aligned}
$$

$\left[M_{A}\right.$ in $\mathrm{kg} ; \mathrm{I}_{C}$ in $\mathrm{kg} \cdot \mathrm{cm}^{2} ; \mathrm{L}_{\mathrm{Cm}}, \mathrm{L}_{A}, \mathrm{~L}, \mathrm{~L}_{0}$, $\ell$ in $\mathrm{cm} ; \quad V$ in $\mathrm{kV} ; \omega_{B_{y}}$, $\omega_{\gamma_{y}}$ in $\mathrm{rad} / \mathrm{s} ; d_{x}$ in $\mu \mathrm{m} ; \tilde{\gamma}_{x}, \hat{\gamma}_{x}, \theta_{x}$ in $\left.\mathrm{rad}\right]$.

The example given in part $i$ of Appendix $P$ shows that this amplitude modulation of the $\beta_{y}$ cosine waveform would also be undetectable for reasonable values of $\theta_{x}, \hat{\gamma}_{x}$ and $d_{x}$, and for a small enough $\tilde{\gamma}_{x}$. 


\section{APPENDIX R}

DYNAMICAL FORCES ON THE SUSPENDED ELECTRODE AS DETECTED BY THE BALANCE

The algebra involved in deriving the equation of motion for the balance beam, using the Principle of Virtual Work and D'Alembert's Principle, was straightforward, but somewhat lengthy. It would be useful to check parts of the result by using Newton's second law of motion.

The equilibrium angle $\hat{\beta}_{y}$, given by Equation $P-14$, agrees with the generalized torque Equation I- 6 if the balance beam is in static equilibrium $\left(Q_{B_{y}}=0\right)$, if $\hat{\beta}_{y}$ is a very small angle, and if the suspended electrode is in static equilibrium $\left(\tilde{\gamma}_{y}=0\right)$. Equation P-14 also agrees with the Newtonian prediction of the torque induced about point (C) of Figures 2-4 when $\tilde{\gamma}_{y}=0$.

The Newtonian prediction for the period of the balance beam is $P_{B_{y}}=2 \pi \sqrt{I C^{\prime K}}$. Using Equations $K-1$ and $P-19$, this equation becomes $P_{B_{y}}=2 \pi / \omega_{B_{y}}$, which agrees with Equation $P-20$.

The amplitude modulations of $\beta_{y}$ can be checked by investigating the time variations of the external forces induced on the suspended electrode. Assume that $\hat{\beta}_{y} \approx 0$, and that either the plane defined by the balance beam knife edges is servoed to gravitational horizon, or that the balance beam freely swings about $\hat{\beta}_{y}=0$ with a very small amplitude $\left(\tilde{\beta}_{y} \approx 0\right)$ when $\tilde{\gamma}_{y}=0$. The suspended electrode is therefore not 
significantly accelerated by the balance beam. Figure 12 shows the remaining external forces that are induced on the moving suspended electrode.

It would be difficult to predict how the tension in the electrode support system gets translated to the knife edge point $(P)$ via the stresses induced in the support rods. So, we will only look at the vertical components of the external forces shown in Figure 12, and will then assume that the balance experiences these vertical forces at the knife edge.

The vertical force due to the radial acceleration is

$$
f_{R}(t)=M_{A} a_{R} \cos \gamma_{y}=10^{-2} M_{A} L_{c m}\left(\dot{\gamma}_{y}\right)^{2} \cos \gamma_{y}
$$

Remembering that

$$
\gamma_{y}=-\tilde{\gamma}_{y} \cos \omega_{\gamma_{y}} t+\hat{\gamma}_{y}
$$

and using the small angle approximation $\cos \gamma_{y}{ }^{\approx 1}$, Equation R-1 becomes

$$
f_{R}(t)=10^{-2} M_{A} L_{c m} \omega^{2}{ }_{\gamma_{y}}^{2}\left(1-\cos ^{2} \omega_{\gamma_{y}} t\right)
$$

$\left[f_{R}\right.$ in $N ; M_{A}$ in $\mathrm{kg} ; L_{\mathrm{cm}}$ in $\mathrm{cm} ; \tilde{\gamma}_{y}$ in $\mathrm{rad} ; \omega_{\gamma_{y}}$ in $\mathrm{rad} / \mathrm{s} ; \mathrm{t}$ in $\mathrm{s}$ ].

The vertical force due to the tangential acceleration is 


$$
f_{T}(t)=M_{A} a_{T} \sin \gamma_{y}=10^{-2} M_{A} L_{c m} \ddot{\gamma}_{y} \sin \gamma_{y}
$$

Using Equation R-2 and the small angle approximation $\sin \gamma_{y} \approx \gamma_{y}$, Equation R-4 becomes

$$
f_{T}(t)=10^{-2} M_{A} L_{c m} \omega_{\gamma_{y}}^{2} \gamma_{y} \cos \omega_{\gamma_{y}} t\left(-\gamma_{y} \cos \omega_{\gamma_{y}} t+\hat{\gamma}_{y}\right)
$$

$\left[f_{T}\right.$ in $N ; M_{A}$ in $\mathrm{kg} ; L_{\mathrm{cm}}$ in $\mathrm{cm} ; \omega_{\gamma_{y}}$ in $\mathrm{rad} / \mathrm{s} ; \tilde{\gamma}_{y}, \hat{\gamma}_{y}$ in $\mathrm{rad} ; \mathrm{t}$ in s].

Adding Equations $R-3$ and $R-5$, and using the identity $2 \cos ^{2} \alpha=(1+\cos 2 \alpha)$ yields

$$
f_{R}(t)+f_{T}(t)=10^{-2} M_{A} L_{c m} \omega_{\gamma_{y}}^{2}\left[-\tilde{\gamma}_{y}^{2} \cos 2 \omega_{\gamma_{y}} t+\tilde{\gamma}_{y} \hat{\gamma}_{y} \cos \omega_{\gamma_{y}} t\right]
$$

where the units are defined in Equations $R-3$ and $R-5$.

The quantities $f_{R}, f_{T}$ and $f_{R}+f_{T}$ are plotted versus time in Figures, 13 and 14 for $V=0 \mathrm{kV}$, (in which case $\hat{\gamma}_{y}=0$ ). The time span covers one pendulum period - which is $\mathrm{P}_{\gamma_{y}}=1.5 \mathrm{~s}$ for a brass suspended electrode with voltage-off.

The torque about the balance center knife is then

$$
-L_{A}\left(f_{R}+f_{T}\right)=+10^{-2} M_{A} L_{c m} L_{A}{ }^{\omega} \gamma_{y}^{2} \tilde{\gamma}_{y}^{2} \cos 2 \omega_{\gamma_{y}} t .
$$


This torque should be - and is - proportional to $\beta_{y}$, which is defined by Equations $P-13, P-21$ and $P-22$. Only the $\cos 2 \omega_{\gamma_{y}} t$ term is present; this is also always the case for a normal balance pan swinging in the absence of horizontal forces. If $\tilde{\gamma}_{y}=3.30 \times 10^{-5} \mathrm{rad}\left(6.8^{\prime \prime}\right)$ and if the mass sensitivity is $0.111 / \mu \mathrm{g}$, then $M_{A}$ would appear to vary by $\pm 5.3 \mu \mathrm{g}$.

The quantities $f_{R}, f_{T}$ and $f_{R}+f_{T}$ are then plotted versus time, for $V=9 \mathrm{kV}$, in Figures 15-16. $P_{\gamma_{y}}$ has increased to $2.8 \mathrm{~s}$ for the brass suspended electrode. The shape of $f_{R}+f_{T}$ depends upon the relative magnitude of $\tilde{\gamma}_{y}$ and $\hat{\gamma}_{y}$, and is described by both the $\cos 2 \omega_{\gamma_{y}} t$ and $\cos \omega_{\gamma_{y}} t$ terris of Equation R-6. If $d_{y}=0$ and $\theta_{y}=5^{\prime \prime}$, then $\hat{\gamma}_{y}=3.30 \times 10^{-5} \mathrm{rad}\left(6.8^{\prime \prime}\right)$, and if $\tilde{\gamma}_{y}=\hat{\gamma}_{y}$ then $f_{R_{x}}+f_{T}=-2.76 \times 10^{-8} \mathrm{~N}$ at $t=(1 / 2) P_{\gamma_{y}}$, giving a torque of $-2.07 \times 10^{-7} \mathrm{~N} \cdot \mathrm{cm}$. If the mass sensitivity is $0.111 / \mu \mathrm{g}$, then $M_{A}$ would appear to be $3.2 \mu \mathrm{g}$ lighter at this instant.

The electrical force, $F_{z}$, is defined by Equations 2-9, 2-10, 2-16 and 2-19:

$$
F_{z}(t)=\left(6.06 \times 10^{-4}\right) v^{2}\left[1+\left(6.5 \times 10^{-9}\right) h^{2}\right]
$$

If $h_{x}=0$ and $\beta_{y}$ is very small, this equation becomes

$$
\begin{aligned}
F_{z}(t) & =\left(6.06 \times 10^{-4}\right) v^{2}+\left(3.94 \times 10^{-12}\right) v^{2}\left[10^{8}\left(L-L_{0} / 2\right)^{2} \gamma_{y}^{2}\right. \\
& +10^{8}\left(\ell-L_{0} / 2\right)^{2} \theta_{y}^{2}+d_{y}^{2}+\left(2 \times 10^{8}\right)\left(L-L_{0} / 2\right)\left(\ell-L_{0} / 2\right) \theta_{y} \gamma_{y} \\
& \left.+\left(2 \times 10^{4}\right)\left(L-L_{0} / 2\right) d_{y} \gamma_{y}+\left(2 \times 10^{4}\right)\left(\ell-L_{0} / 2\right) d_{y} \theta_{y}\right] .
\end{aligned}
$$


Substituting Equation R-2 for $\gamma_{y}$, using the identity $\cos ^{2} \alpha=(1 / 2)(1+\cos 2 \alpha)$, and gathering the constant and time-dependent terms into groups gives

$$
\begin{aligned}
F_{z}(t) & =\left\{\left(6.06 \times 10^{-4}\right) v^{2}+\left(3.94 \times 10^{-12}\right) v^{2}\left[10^{8}\left(\ell-L_{0} / 2\right)^{2} \theta_{y}^{2}+d d_{y}^{2}\right.\right. \\
& +\left(2 \times 10^{4}\right)\left(\ell-L_{0} / 2\right) d_{y} \theta_{y}+10^{8}\left(L-L_{0} / 2\right)^{2}\left(\hat{\gamma}_{y}^{2}+\tilde{\gamma}_{y}^{2} / 2\right) \\
& \left.\left.+\left(2 \times 10^{8}\right)\left(L-L_{0} / 2\right)\left(\ell-L_{o} / 2\right) \theta_{y} \hat{\gamma}_{y}+\left(2 \times 10^{4}\right)\left(L-L_{0} / 2\right) d_{y} \hat{\gamma}_{y}\right]\right\} \\
& +\left\{( 3 . 9 4 \times 1 0 ^ { - 1 2 } ) v ^ { 2 } \left[10^{8}\left(L-L_{0} / 2\right)^{2}\left(\tilde{\gamma}_{y}^{2} / 2\right) \cos 2 \omega_{\gamma_{y}} t\right.\right. \\
& -\left(2 \times 10^{8}\right)\left(L-L_{0} / 2\right)^{2} \tilde{\gamma}_{y} \hat{\gamma}_{y} \cos \omega_{\gamma_{y}} t \\
& -\left(2 \times 10^{4}\right)\left(L-L_{0} / 2\right) d_{y} \tilde{\gamma}_{y} \cos \omega_{\gamma_{y}} t \\
& \left.\left.-\left(2 \times 10^{8}\right)\left(L-L_{0} / 2\right)\left(\ell-L_{o} / 2\right) \theta_{y} \tilde{\gamma}_{y} \cos \omega_{\gamma_{y}} t\right]\right\}
\end{aligned}
$$

$\left[F_{z}\right.$ in $N ; L, \ell, L_{0}$ in $\mathrm{cm} ; d_{y}$ in $\mu \mathrm{ml} ; V$ in $\mathrm{kV} ; \omega_{\gamma_{y}}$ in $\mathrm{rad} / \mathrm{s} ; \theta_{y}$, $\hat{\gamma}_{y}, \tilde{\gamma}_{y}$ in rad; $t$ in $\left.s\right]$

or

$$
F_{Z}(t)=f_{c}+f_{Z}(t)
$$

where $f_{C}$ is the constant term in Equation $R-7$ and $f_{z}$ is the time-dependent term.

The equilibrium angle, $\hat{\beta}_{y}$, for the balance beam should depend upon the net, time-independent, torque, $\hat{\Gamma}_{\beta_{y}}$, about the center knife, and upon the balance beam stiffness, $K$ : 


$$
\hat{\beta}_{y}=\hat{\Gamma}_{\hat{\beta}_{y}} / K=\left[M_{B} g L_{B}-M_{A} g L_{A}-f_{C} L_{A}\right] / K \text {. }
$$

This equation agrees exactly with Equation $\mathrm{P}-14$, and thus verifies the presence of the $(1 / 2) \tilde{\gamma}_{y}^{2}$ quantity in that equation.

The quantity $f_{z}$ is plotted versus time in Figure 17 using Equations $R-7$ and $R-8$ for the brass suspended electrode at $V=9 \mathrm{kV}$ with $d_{x}=d_{y}=\theta_{x}=0$ and $\theta_{y}=5 "$. The amplitudes of oscillation would be zero if either $V=0$ or if $\tilde{\gamma}_{y}=0$.

The total time-dependent torque about the balance center knife, $-L_{A}\left(f_{R}+f_{T}+f_{z}\right)$, is proportional to $\beta_{y}$. Substitution of Equations $R-6$ $\mathrm{R}-8$ into this equation gives agreement with the numerators of the modulation terms of Equations P-5, which are defined by Equations $\mathrm{P}-21$ and $P-22$. So, the amplitude modulations of $B_{y}$ arise from the time-dependent vertical forces $f_{R}, f_{T}$ and $f_{Z}$.

It is useful to express the time-dependent torque in terms of the mass variation of the mass $M_{A}$. Assuming that the mass sensitivity is calibrated using sensitivity weights with voltage-off, then the mass variation, $\Delta m$, is

$$
\Delta m=-L_{A}\left(f_{R}+f_{T}+f_{Z}\right)\left(1 / K^{(0 N)}\right)\left(" / 4.85 \times 10^{-6} \mathrm{rad}\right)\left(\mu g / 0.11^{\prime \prime}\right)
$$

[ $\Delta \mathrm{m}$ in $\mu g ; L_{A}$ in $\mathrm{cm} ; \mathrm{f}_{\mathrm{R}}, \mathrm{f}_{\mathrm{T}}, \mathrm{f}_{Z}$ in $\mathrm{N} ; \quad K$ in $\left.\mathrm{N} \cdot \mathrm{cm} / \mathrm{rad}\right]$. which for the brass electrode at $V=9 \mathrm{kV}$ is 


$$
\Delta m=\left(1.14 \times 10^{8}\right)\left(f_{R}+f_{T}+f_{Z}\right)
$$

$\left[\Delta \mathrm{m}\right.$ in $\mu g ; f_{R}, f_{T}, f_{z}$ in $\left.N\right]$.

Figure 18 shows $\Delta \mathrm{m}$ versus time for a brass electrode at $\mathrm{V}=9 \mathrm{kV}$ and $\theta_{y}=5 "$. This figure was obtained by adding the curves in Figures 16 and 17 and then using Equation R-10. It predicts that, for this example, $\Delta \mathrm{m}$ varies from $-30.7 \mu \mathrm{g}$ to $+41.3 \mu \mathrm{g}$ every $2.8 \mathrm{~s}$; but the prediction for this same example in part $i$ of Appendix $P$ is of a $\Delta m$ variation between $-0.13 \mu \mathrm{g}$ and $+0.14 \mu \mathrm{g}$. However, if, somehow, the period of the pendulum, $P_{\gamma_{y}}$, could be made very long compared with the $P_{\beta_{y}}^{(O N)}=46.8 \mathrm{~s}$ balance beam period (so that $\omega_{\beta_{y}} \gg \omega_{\gamma_{y}}$ ), then Equations $P-21$ and $P-22$ also predict the same $\Delta m$ variation as that of Figure 18 because $K^{(O N)}=10^{-2} I_{C} \omega_{B_{y}}^{2}$.

The balance acts as a low-pass frequency filter; the $2.8 \mathrm{~s}$ period of the pendulum vertical force variations is too short for the $46.8 \mathrm{~s}$ balance beam period to respond to, and thus these variations are attenuated. So, the amplitude modulations of the $\beta_{y}$ cosine waveform are very small. The equilibrium angle $\hat{\beta}_{y}$, however, is directly affected by the amplitude squared $\left(\tilde{\gamma}_{y}^{2}\right)$ of the pendulum motion. 


\section{APPENDIX $S$}

DYNAMICAL CAPACITANCE VARIATIONS

The capacitance is defined by Equations 2-9, 2-10, 2-12 and 2-13 as

$$
C(t)=C\left(z_{L}\right)\left[1+\left(6.5 \times 10^{-9}\right) h^{2}\right]
$$

If $h_{x}=0$ and $\beta_{y}$ is very small, this equation becomes

$$
\begin{aligned}
C(t)= & C\left(z_{L}\right)+C\left(z_{L}\right)\left(6.5 \times 10^{-9}\right)\left[10^{8}\left(L-L_{0} / 2\right)^{2} \gamma_{y}^{2}+10^{8}\left(\ell-L_{0} / 2\right)^{2} \theta_{y}^{2}\right. \\
& +d_{y}^{2}+\left(2 \times 10^{8}\right)\left(L-L_{0} / 2\right)\left(\ell-L_{0} / 2\right) \theta_{y} \gamma_{y} \\
& \left.+\left(2 \times 10^{4}\right)\left(L-L_{0} / 2\right) d_{y} \gamma_{y}+\left(2 \times 10^{4}\right)\left(\ell-L_{0} / 2\right) \theta_{y} d_{y}\right] .
\end{aligned}
$$

But $\gamma_{y}=-\tilde{\gamma}_{y} \cos \omega_{\gamma_{y}} t+\hat{\gamma}_{y}$, and $\hat{\gamma}_{y} \approx 0$ for the $v_{\text {rms }}=100 \mathrm{~V}$ value at which the capacitance measurements are made. Therefore, using the identity $\cos ^{2} \alpha=(1 / 2)(1+\cos 2 \alpha)$, and gathering the constant and time-dependent terms into groups gives

$$
\begin{aligned}
C(t)= & \left\{C\left(z_{L}\right)+(0.325) C\left(z_{L}\right)\left(L-L_{0} / 2\right)^{2} \tilde{\gamma}_{y}^{2}\right. \\
& +(0.65) C\left(z_{L}\right)\left(\ell-L_{0} / 2\right)^{2} \theta_{y}^{2}+\left(6.5 \times 10^{-9}\right) C\left(z_{L}\right) d_{y}^{2} \\
& \left.+\left(1.3 \times 10^{-4}\right) C\left(z_{L}\right)\left(\ell-L_{0} / 2\right) \theta_{y} d_{y}\right\} \\
& +\left\{(0.325) C\left(z_{L}\right)\left(L-L_{0} / 2\right)^{2} \tilde{\gamma}_{y}^{2} \cos 2 \omega_{\gamma_{y}} t\right. \\
& -(1.3) C\left(z_{L}\right)\left(L-L_{0} / 2\right)\left(\ell-L_{0} / 2\right) \theta_{y} \tilde{\gamma}_{y} \cos \omega_{\gamma_{y}} t
\end{aligned}
$$




$$
\left.-\left(1.3 \times 10^{-4}\right) C\left(z_{L}\right)\left(L-L_{0} / 2\right) d_{y} \tilde{\gamma}_{y} \cos \omega_{\gamma_{y}} t\right\}
$$

[C in pF; $z_{L}, L, \ell, L_{0}$ in $\mathrm{cm} ; d_{y}$ in $\mu \mathrm{m} ; \omega_{\gamma_{y}}$ in $\mathrm{rad} / \mathrm{s} ; \theta_{y}, \tilde{\gamma}_{y}$ in rad; $t$ in $s]$.

So, the fractional change in $C(t)$ compared with $C\left(z_{L}\right)$ is

$$
\begin{aligned}
\left\{C(t) / C\left(z_{L}\right)-1\right\}=\left\{\left[(0.65)\left(\ell-L_{0} / 2\right)^{2} \theta_{y}^{2}+\left(6.5 \times 10^{-9}\right) d_{y}^{2}\right.\right. \\
\left.\left.\quad+\left(1.3 \times 10^{-4}\right)\left(\ell-L_{0} / 2\right) \theta_{y} d_{y}\right]+(0.325)\left(L-L_{0} / 2\right)^{2} \tilde{\gamma}_{y}^{2}\right\} \\
\quad+\left\{(0.325)\left(L-L_{0} / 2\right)^{2} \tilde{\gamma}_{y}^{2} \cos 2 \omega_{\gamma_{y}} t-(1.3)\left(L-L_{0} / 2\right)\left(\ell-L_{0} / 2\right) \theta_{y} \tilde{\gamma}_{y} \cos \omega_{\gamma_{y}} t\right. \\
\left.\quad-\left(1.3 \times 10^{-4}\right)\left(L-L_{0} / 2\right) d_{y} \tilde{\gamma}_{y} \cos \omega_{\gamma_{y}} t\right\}
\end{aligned}
$$

or

$$
\delta C(t) / C\left(z_{L}\right)=\varepsilon_{C}+\varepsilon_{t}(t)
$$

where $\varepsilon_{c}$ is the constant term in Equation S-3 and $\varepsilon_{t}$ is the time-dependent term. It is assumed that the amplitude of the $\gamma_{y}$ motion decreases due to damping at a rate which is long compared with the pendulum period. Therefore, the $(0.325)\left(L-L_{0} / 2\right)^{2} \tilde{\gamma}_{y}^{2}$ term is included in $\varepsilon_{c}$.

$\delta C(t) / C\left(z_{L}\right)$ is plotted versus time in Figure 19 for $C\left(z_{L}\right)=100 \mathrm{pF}$ and $d_{x}=d_{y}=\theta_{x}=0$. An analogous plot occurs for pendulum motion in the $x-z$ plane. A small time constant and a fast enough recording device must be used for the capacitance bridge detector in order to 
respond correctly to the $\mathrm{P}_{\gamma_{y}}=1.5 \mathrm{~s}$ period of a brass electrode. If the time constant is too long, then the detector will begin averaging the signal - thus resulting in large systematic errors.

The signal shape depends upon the relative electrode equilibrium alignments and upon the pendulum amplitude. Perfect alignment yields a pure $\cos 2 \omega_{\gamma_{y}} t$ signal. The $\cos \omega_{\gamma_{y}}$ component becomes more enhanced with increasing electrode misalignments and relative displacements.

The static equilibrium values of $\delta C / C$ are $+0.34 \mathrm{ppm}$ and $0 \mathrm{ppm}$ for $\theta_{y}=5^{\prime \prime}$ and $\theta_{y}=0$, respectively. Therefore, the minimum reading of $\delta c / C$ is the correct one only when $\hat{h}_{x}=\hat{h}_{y}=0$, but it provides the best estimate of any of the simple options. The pendulum amplitude must be quite small when making the capacitance measurements - and, the larger the electrode misalignments and relative displacements, the smaller this amplitude must be. 
a) Method used to estimate the voltage ratio uncertainty.

The voltage ratio, $V_{c a l c} / V_{\text {meas }}$, which can be obtained from Equation $4-4$, is

$$
v_{\text {calc }} / V_{\text {meas }}=\left(1 / V_{\text {meas }}\right)\left[-\left(2 \times 10^{4}\right) \hat{F}_{z} /\left(\partial \hat{C} / \partial z_{L}\right) \hat{h}_{x}, \hat{h}_{y}\right]^{1 / 2}
$$

$\left[F_{z}\right.$ in $N$; $V_{\text {calc }}, V_{\text {meas }}$ in $k V ; C$ in $p F ; h$ in $\mu \mathrm{m} ; z_{L}$ in $\left.\mathrm{cm}\right]$.

The quantities in Equation T-1 that have errors associated with them are $v_{\text {meas }}, \hat{F}_{Z}, \hat{C}$ and $z_{L}$. There is an error involved in measuring the capacitance at low voltage rather than at the high voltages, which must be included in $\hat{C}$ (along with the dynamical measurement errors and any errors resulting from temperature fluctuations).

There is a random error in determining $m$, which (according to Equation $\mathrm{P}-18$ ) should be included in the uncertainty of $\hat{F}_{z}$ - along with the systematic static and dynamic errors. If there are large curvatures and changes in the functional form of $\hat{C}$ versus $z_{L}$ then the uncertainty in the slope will increase - in addition to the error involved in not determining the slope at constant $h$. 
The static systematic error in $\hat{F}_{z}$ is correlated with the low voltage error in measuring $\hat{C}$, and also with the error involved in not determining the slope at constant $h$. The uncertainties of Equation T-1 are therefore correlated mixtures of random and systematic errors.

Rather than attempting to assign correlation coefficients and deciding how to combine random and systematic errors for nonexistent data, only measured quantities and their assumed measurement uncertainties will be used. The voltage ratio will be calculated for a variety of carriage scans, using the low-voltage capacitance values and neglecting the fact that the slope should be determined at constant $h$. The correlation effects and the effects due to the unmeasurable quantities should thereby be reflected in this uncertainty estimate.

b) Calculations for Type I scans with a brass electrode at $V=9 \mathrm{kV}$.

Example 1. Perfectly aligned right circular cylinders with no measurement errors.

Let mass $m$ be such that $\hat{F}_{z}=m g$ at $V=9 k V$ when $\hat{h}=0$. Since $\hat{h}=0$, Equation 4-2 becomes

$$
\hat{F}_{Z}=m g=\left(6.06 \times 10^{-4}\right)(9 \mathrm{kV})^{2}=4.9086 \times 10^{-2} \mathrm{~N} .
$$


Since the path is linear, and $\hat{F}_{Z}$ is assumed to be equal to mg when $\hat{\beta}_{y}^{(O N)}=\hat{\beta}_{y}^{(O F F)}$ in the force determination measurement, Equation 4-1 becomes

$$
\begin{aligned}
v_{c a 1 c}^{2} & =-\left(2 \times 10^{4}\right) \mathrm{mg}\left(\Delta \mathrm{z}_{\mathrm{L}} / \Delta \mathrm{C}\right)=-\left(2 \times 10^{4}\right)\left(4.9086 \times 10^{-2} \mathrm{~N}\right)(2.5 \mathrm{~cm}) / \\
& (69.7 \mathrm{pF}-100 \mathrm{pF})
\end{aligned}
$$

or $V_{\text {calc }}=9 \mathrm{kV}=V_{\text {meas }}$. Therefore, there is no error in this example.

Example 2. A -2 ppm error in $\hat{F}_{z}$ and no other errors.

The static and dynamic errors are such that $\hat{F}_{z}$ appears to be 2 ppm smaller than $m g$ when $\hat{F}_{z}$ actually equals $\mathrm{mg} . \hat{\mathrm{F}}_{\mathrm{z}}$ would thus be increased by 2 ppm until $\hat{\beta}_{y}^{(O N)}=\hat{\beta}_{y}^{(O F F)}$, so that

$$
v_{\text {meas }}^{2}=\left(4.9086 \times 10^{-2} \mathrm{~N}\right)\left[1+\left(2 \times 10^{-6}\right)\right] /\left(6.06 \times 10^{-4}\right)
$$

or

$$
V_{\text {meas }}=9.000009 \mathrm{kV} \text {. }
$$

$V_{\text {calc }}$ is the same as that for Example 1; therefore, $V_{\text {calc }}$ is $1 \mathrm{ppm}$ smaller than $V_{\text {meas }}$. 
Example 3. A -2 ppm error in $\hat{F}_{z}$, the interferometer errors, but no other errors.

The smallest value of $\Delta z_{L}$ that might be measured by the laser inteferometer system is $\left[2.5 \mathrm{~cm}-\left(5 \times 10^{-6}\right) \mathrm{cm}\right]$ or $(2.5 \mathrm{~cm})\left[1-\left(2 \times 10^{-6}\right)\right]$, so

$$
\begin{aligned}
v_{\text {calc }}^{2} & =-\left(2 \times 10^{4}\right)\left(4.9086 \times 10^{-2} \mathrm{~N}\right)(2.5 \mathrm{~cm})\left[1-\left(2 \times 10^{-6}\right)\right] / \\
& {[69.7 \mathrm{pF}-100 \mathrm{pF}] }
\end{aligned}
$$

or

$$
V_{\text {calc }}=8.999991 \mathrm{kV} \text {. }
$$

$V_{\text {meas }}=9.000009 \mathrm{kV}$; therefore $V_{\text {calc }}$ is 2 ppm smaller than $V_{\text {meas }}$.

Example 4. A -2 ppm error in $\hat{F}_{Z}$; all other errors except the temperature effects.

$$
\begin{aligned}
v_{\text {calc }}^{2} & =-\left(2 \times 10^{4}\right)\left(4.9086 \times 10^{-2} \mathrm{~N}\right)(2.5 \mathrm{~cm})\left[1-\left(2 \times 10^{-6}\right)\right] / \\
& \left\{[69.7 \mathrm{pF}-100 \mathrm{pF}]\left(1+10^{-6}\right)\right\}
\end{aligned}
$$

or

$$
V_{\text {calc }}=8.9999862 \mathrm{kV} \text {. }
$$


$V_{\text {meas }}=9.000009 \mathrm{kV}$; therefore $V_{\text {calc }}$ is $2.6 \mathrm{ppm}$ smaller than $V_{\text {meas }}$.

Example 5. A -2 ppm error in $\hat{F}_{z}$ and all errors included.

$$
\begin{aligned}
V_{\text {calc }}^{2} & =-\left(2 \times 10^{4}\right)\left(4.9086 \times 10^{-2} \mathrm{~N}\right)(2.5 \mathrm{~cm})\left[1-\left(2 \times 10^{-6}\right)\right] / \\
& \left\{\left[(69.7 \mathrm{pF})\left(1-10^{-6}\right)-(100 \mathrm{pF})\left(1+10^{-6}\right)\right]\left(1+10^{-6}\right)\right\}
\end{aligned}
$$

or

$$
V_{\text {calc }}=8.9999609 \mathrm{kV} \text {. }
$$

$V_{\text {meas }}=9.000009 \mathrm{kV}$; therefore $V_{\text {calc }}$ is $5.4 \mathrm{ppm}$ smaller than $V_{\text {meas }}$ when $V_{\text {calc actually equals }} V_{\text {meas. }}$. It is not likely, however, that the errors would all combine in this manner. The combination given in Example 4 is a more likely choice for the maximum error in determining $\mathrm{v}_{\text {calc }}$.

Example 6. A +2 ppm error in $\hat{F}_{z}$ and no other errors.

The static and dynamic errors are such that $\hat{F}_{z}$ appears to be $2 \mathrm{ppm}$ larger than mg when $\hat{F}_{z}$ actually equals $\mathrm{mg}$. $\hat{\mathrm{F}}_{\mathrm{z}}$ would thus be decreased by 2 ppm until $\hat{\beta}_{y}^{(O N)}=\hat{\beta}_{y}^{(O F F)}$, so that

$$
V_{\text {meas }}^{2}=\left(4.9086 \times 10^{-2} N\right)\left[1-\left(2 \times 10^{-6}\right)\right] /\left(6.06 \times 10^{-4}\right)
$$

or 
$V_{\text {meas }}=8.999991 \mathrm{kV}$.

$V_{\text {calc }}=9.0000000 \mathrm{kV}$; therefore $V_{\text {calc }}$ is $1 \mathrm{ppm}$ larger than $V_{\text {meas }}$.

Example $7 . A+2 \mathrm{ppm}$ error in $\hat{\mathrm{F}}_{\mathrm{z}}$; the interferometer errors, but no other errors.

The largest value of $\Delta z_{L}$ that might be measured by the laser inteferometer system is $\left[2.5 \mathrm{~cm}+\left(5 \times 10^{-6}\right) \mathrm{cm}\right]$ or $(2.5 \mathrm{~cm})\left[1+\left(2 \times 10^{-6}\right) \mathrm{cm}\right]$, so

$$
\begin{aligned}
& V_{\text {calc }}^{2}=-\left(2 \times 10^{4}\right)\left(4.9086 \times 10^{-2} \mathrm{~N}\right)(2.5 \mathrm{~cm})\left[1+\left(2 \times 10^{-6}\right)\right] / \\
& {[69.7 \mathrm{pF}-100 \mathrm{pF}]}
\end{aligned}
$$

or

$$
V_{\text {calc }}=9.000009 \mathrm{kV} \text {. }
$$

$\mathrm{V}_{\text {meas }}=8.999991 \mathrm{kV}$; therefore $\mathrm{V}_{\text {calc }}$ is $2 \mathrm{ppm}$ larger than $\mathrm{V}_{\text {meas }}$.

Example 8. A $+2 \mathrm{ppm}$ error in $\hat{F}_{z}$; all other errors except the temperature effects.

$$
\begin{aligned}
v_{\text {calc }}^{2} & =-\left(2 \times 10^{4}\right)\left(4.9086 \times 10^{-2} \mathrm{~N}\right)(2.5 \mathrm{~cm})\left[1+\left(2 \times 10^{-6}\right)\right] / \\
& \left\{[69.7 \mathrm{pF}-100 \mathrm{pF}]\left(1+10^{-6}\right)\right\}
\end{aligned}
$$


$V_{\text {calc }}=9.0000043 \mathrm{kV}$.

$V_{\text {meas }}=8.999991 \mathrm{kV}$; therefore $\mathrm{V}_{\text {calc }}$ is $1.5 \mathrm{ppm}$ larger than $\mathrm{V}_{\text {meas }}$.

Example 9. A +2 ppm error in $\hat{F}_{z}$ and all errors included.

$$
\begin{aligned}
V_{\text {calc }}^{2}= & -\left(2 \times 10^{4}\right)\left(4.9086 \times 10^{-2} \mathrm{~N}\right)(2.5 \mathrm{~cm})\left[1+\left(2 \times 10^{-6}\right)\right] / \\
& \left\{\left[(69.7 \mathrm{pF})\left(1+10^{-6}\right)-(100 \mathrm{pF})\left(1-10^{-6}\right)\right]\left(1+10^{-6}\right)\right\}
\end{aligned}
$$

or

$V_{\text {calc }}=9.0000339 \mathrm{kV}$.

$V_{\text {meas }}=8.9999908 \mathrm{kV}$; therefore $V_{\text {calc }}$ is $4.7 \mathrm{ppm}$ larger than $V_{\text {meas }}$.

However, it is again unlikely that the errors would all combine in this manner.

A reasonable choice of the maximum uncertainty in $\mathrm{V}_{\text {calc }}$ for a Type $\mathrm{I}$ scan might be best given by Examples 4 and 8: $V_{\text {calc }}$ can be determined to within $+1.5 \mathrm{ppm}$ and $-2.5 \mathrm{ppm}$ for a scan known to be along the combined electrodes symmetry axis. 
c) Calculations for Type I scans with an aluminum electrode at $\mathrm{V}=4.02 \mathrm{kV}$.

Example 10. A -2 ppm error in $\hat{F}_{z}$ and all errors except the temperature effects.

Let mass $m$ be such that $\hat{F}_{z}=m g$ at $V=4.02 k V$ when $\hat{h}=0$. Since $\hat{h}=0$, Equation 4-2 becomes

$$
\hat{\mathrm{F}}_{z}=m g=\left(6.06 \times 10^{-4}\right)(4.02 \mathrm{kV})^{2}=9.7932024 \times 10^{-3} \mathrm{~N} .
$$

$\hat{F}_{z}$ appears to be $2 \mathrm{ppm}$ smaller than this value for $\mathrm{mg}$; therefore, the voltage is adjusted until

$$
V_{\text {meas }}^{2}=\left(9.7932024 \times 10^{-3} N\right)\left[1+\left(2 \times 10^{-6}\right)\right] /\left(6.06 \times 10^{-4}\right)
$$

or

$$
\begin{aligned}
V_{\text {meas }} & =4.020004 \mathrm{kV} . \\
V_{\text {calc }}^{2} & =-\left(2 \times 10^{4}\right)\left(9.7932024 \times 10^{-3} \mathrm{~N}\right)(2.5 \mathrm{~cm})\left[1-\left(2 \times 10^{-6}\right)\right] / \\
& \quad\left\{[69.7 \mathrm{pF}-100 \mathrm{pF}]\left(1+10^{-6}\right)\right\}
\end{aligned}
$$

or 


$$
V_{\text {calc }}=4.0199937 \mathrm{kV} \text {. }
$$

Therefore, $V_{c a l c}$ is $2.6 \mathrm{ppm}$ smaller than $V_{\text {meas }}$ when $V_{c a l c}$ actually equals $V_{\text {meas }}$ when there are no errors.

Example 11. A $\quad$ +2 ppm error in $\hat{F}_{z}$ and all errors except the temperature effects.

$\hat{F}_{\mathrm{z}}$ appears to be $2 \mathrm{ppm}$ larger than $\mathrm{mg}$; therefore, the voltage is adjusted until

$$
V_{\text {meas }}^{2}=\left(9.7932024 \times 10^{-3} N\right)\left[1-\left(2 \times 10^{-6}\right)\right] /\left(6.06 \times 10^{-4}\right)
$$

or

$$
\begin{aligned}
V_{\text {meas }}= & 4.019996 \mathrm{kV} . \\
V_{\text {ca } 1 \mathrm{c}}^{2}= & -\left(2 \times 10^{4}\right)\left(9.7932024 \times 10^{-3} \mathrm{~N}\right)(2.5 \mathrm{~cm})\left[1+\left(2 \times 10^{-6}\right)\right] / \\
& \left\{[69.7 \mathrm{pF}-100 \mathrm{pF}]\left(1+10^{-6}\right)\right\}
\end{aligned}
$$

or

$V_{\text {calc }}=4.020002 \mathrm{kV}$.

Therefore, $V_{\text {calc }}$ is $1.5 \mathrm{ppm}$ larger than $V_{\text {meas }}$. 
Examples 10 and 11 agree with Examples 4 and 8, as expected, because it should not matter which electrode is used, at what voltage, as long as the carriage is translated along the combined electrode symmetry axis. The voltage, of course, must be such that the suspended electrode system is in stable mechanical equilibrium.

Example 12. A $A$ ppm error in $\hat{F}_{z}$; the interferometer errors, and a dynamical capacitance error at $\mathrm{C}=69.7 \mathrm{pF}$.

$$
\begin{aligned}
V_{\text {calc }}^{2} & =-\left(2 \times 10^{4}\right)\left(9.7932024 \times 10^{-3} \mathrm{~N}\right)(2.5 \mathrm{~cm})\left[1+\left(2 \times 10^{-6}\right)\right] / \\
& {\left[(69.7 \mathrm{pF})\left(1+10^{-6}\right)-100 \mathrm{pF}\right] }
\end{aligned}
$$

or

$$
V_{\text {calc }}=4.0200078 \mathrm{kV} \text {. }
$$

$V_{\text {meas }}=4.0199958 \mathrm{kV}$; therefore $V_{\text {calc }}$ is $2.9 \mathrm{ppm}$ larger than $V_{\text {meas }}$ as compared with $1.5 \mathrm{ppm}$ larger in Example 11. However, the capacitance would be measured at many points in order to find the best least-squares fit of the straight line in this example. Therefore, it is more appropriate to include the dynamical capacitance error in all the measurements for the slope method. 
d) Calculations for Type II scans with a brass electrode at V=9 kV and $\theta_{y}=5 "$.

Example 13. $\hat{F}_{z}$ determined at $C\left(z_{L}\right)=100 \mathrm{pF}$ with no measurement errors.

Table 7 lists the actual value of $\hat{F}_{z}$ as being $4.7 \mathrm{ppm}$ larger than the value for mg. Thus the voltage must be adjusted until

$$
v_{\text {meas }}^{2}=\left(4.9086 \times 10^{-2} N\right)\left[1-\left(4.7 \times 10^{-6}\right)\right] /\left(6.06 \times 10^{-4}\right)
$$

or

$$
V_{\text {meas }}=8.9999787 \mathrm{kV} \text {. }
$$

The capacitance, from Equation 2-12, is

$$
\hat{C}\left(\hat{h}, z_{L}\right)=C\left(z_{L}\right)\left[1+\left(6.5 \times 10^{-9}\right) \hat{h}^{2}\right]
$$

where, from Equation $2-10, \hat{h}$ is

$$
\hat{h}_{y}=10^{4}\left[\ell-L_{o}\left(z_{L}\right) / 2\right] \theta_{y}
$$

because $\hat{\gamma}_{y} \approx 0$ at $V_{r m s}=100 \mathrm{~V}$. $L_{0}\left(z_{L}\right)=10 \mathrm{~cm}$ at $C\left(z_{L}\right)=100 p F$, so $h=7.275 \mu \mathrm{m}$ and $\Delta C / C=+0.34$ ppm at $z_{L}=0 \mathrm{~cm}$, whereas $C\left(z_{L}\right)=69.7 p F, L_{0}\left(z_{L}\right)=7.5 \mathrm{~cm}$ $h=7.58 \mu \mathrm{m}$ and $\Delta C / C=+0.37 \mathrm{ppm}$ at $z_{L}=2.5 \mathrm{~cm}$. Therefore the capacitance will be approximately $+0.3 \mathrm{ppm}$ larger than the $C\left(z_{L}\right)$ value at every $z_{L}$ point. 


$$
\begin{aligned}
v_{\text {calc }}^{2}= & -\left(2 \times 10^{4}\right)\left(4.9086 \times 10^{-2} \mathrm{~N}\right)(2.5 \mathrm{~cm}) / \\
& \left\{[69.7 \mathrm{pF}-100 \mathrm{pF}]\left[1+\left(0.3 \times 10^{-6}\right)\right]\right\}
\end{aligned}
$$

or

$$
V_{\text {calc }}=8.9999985 \mathrm{kV} \text {. }
$$

$V_{\text {meas }}=8.9999787 \mathrm{kV}$; therefore $V_{\text {calc }}$ is $2.2 \mathrm{ppm}$ larger than $V_{\text {meas }}$ with no experimental errors present. This discrepancy arises because the force is measured at $9 \mathrm{kV}$ (so that the suspended electrode has swung out to an equilibrium angle of $\hat{\gamma}_{y}=6.8^{\prime \prime}$, causing a $4.7 \mathrm{ppm}$ increase in the actual capacitance), but the capacitance is measured at $V_{r m s}=100 \mathrm{~V}$ (resulting in only a $0.3 \mathrm{ppm}$ capacitance increase). No error would occur in this example if the capacitance could be measured at $V=9 \mathrm{kV}$.

Example 14. $\hat{F}_{z}$ determined at $C\left(z_{L}\right)=69.7 \mathrm{pF}$ with no measurement errors.

The value of $L_{0}\left(z_{L}\right)$ is smaller at $z_{L}=2.5 \mathrm{~cm}$ than at $z_{L}=0 \mathrm{~cm}$. Therefore, the value of $\hat{h}_{y}$, as calculated from Equation 2-10, is also smaller - which then results in a smaller value for $\hat{F}_{z}$. Table 9 lists the relevant quantities for this Type II scan with a brass electrode. 


$$
\begin{aligned}
v_{\text {calc }}^{2}= & -\left(2 \times 10^{4}\right)\left(4.9086 \times 10^{-2} \mathrm{~N}\right)(2.5 \mathrm{~cm}) /\{[69.7 \mathrm{pF}-100 \mathrm{pF}] \mathrm{X} \\
& {\left.\left[1+\left(0.3 \times 10^{-6}\right)\right]\right\} }
\end{aligned}
$$

or

$\mathrm{V}_{\text {calc }}=8.9999985 \mathrm{kV}$.

$V_{\text {meas }}=8.9999923 \mathrm{kV}$; therefore $V_{\text {calc }}$ is $0.6 \mathrm{ppm}$ larger than $V_{\text {meas }}$ at $\mathrm{C}=69.7 \mathrm{pF}$. The discrepancy is less than at $\mathrm{C}=100 \mathrm{pF}$, but $\hat{\mathrm{F}}_{\mathrm{z}}$ should be determined at many points along the scan.

Example 17. A -2 ppm error in $\hat{F}_{z}$ at $C\left(z_{L}\right)=100 \mathrm{pF}$ and all except the temperature effects.

$$
\begin{aligned}
v_{\text {carc }}^{2}= & -\left(2 \times 10^{4}\right)\left(4.9086 \times 10^{-2} \mathrm{~N}\right)(2.5 \mathrm{~cm})\left[1-\left(2 \times 10^{-6}\right)\right] / \\
& \left\{[69.7 \mathrm{pF}-100 \mathrm{pF}]\left[1+\left(1.3 \times 10^{-6}\right)\right]\right\}
\end{aligned}
$$

or

$$
V_{\text {calc }}=8.9999848 \mathrm{kV}
$$

$V_{\text {meas }}=8.9999878 \mathrm{kV}$; therefore $V_{c a l c}$ is $0.4 \mathrm{ppm}$ smaller than $\mathrm{V}_{\text {meas }}$.

Example 18. $A+2$ ppm error in $\hat{F}_{z}$ at $C\left(z_{L}\right)=100 p F$ and all errors except the temperature effects. 


$$
\begin{aligned}
v_{\text {calc }}^{2}= & -\left(2 \times 10^{4}\right)\left(4.9086 \times 10^{-2} \mathrm{~N}\right)(2.5 \mathrm{~cm})\left[1+\left(2 \times 10^{-6}\right)\right] / \\
& \left\{[69.7 \mathrm{pF}-100 \mathrm{pF}]\left[1+\left(1.3 \times 10^{-6}\right)\right]\right\}
\end{aligned}
$$

or

$$
V_{\text {calc }}=9.0000029 \mathrm{kV} \text {. }
$$

$V_{\text {meas }}=8.9999698 \mathrm{kV}$; therefore $V_{\text {calc }}$ is $3.6 \mathrm{ppm}$ larger than $V_{\text {meas }}$.

Example 19. A -2 ppm error in $\hat{F}_{z}$ at $C\left(z_{L}\right)=69.7 \mathrm{pF}$ and a11 errors except the temperature effects.

$V_{\text {calc }}=8.9999848 \mathrm{kV}$ and $V_{\text {meas }}=9.0000013 \mathrm{kV}$; therefore $V_{\text {calc }}$ is 1.9 ppm smaller than $V_{\text {meas }}$.

Example 20. $A+2$ ppm error in $\hat{F}_{z}$ at $C\left(z_{L}\right)=69.7 \mathrm{pF}$ and all errors except the temperature effects.

$V_{\text {calc }}=9.0000029 \mathrm{kV}$ and $V_{\text {meas }}=8.9999833 \mathrm{kV}$; therefore $V_{\text {calc }}$ is 2.1 ppm larger than $v_{\text {meas }}$.

e) Calculations for Type II scans with an aluminum electrode at $V=4.02 \mathrm{kV}$ and $\theta_{\mathrm{y}}=5^{\prime \prime}$. 
Example 21. A -2 ppm error in $\hat{F}_{z}$ at $C\left(z_{L}\right)=100 \mathrm{pF}$ and all errors except the temperature effects.

$$
\begin{aligned}
v_{\text {calc }}^{2}= & -\left(2 \times 10^{4}\right)\left(9.7932024 \times 10^{-3} \mathrm{~N}\right)(2.5 \mathrm{~cm})\left[1-\left(2 \times 10^{-6}\right)\right] / \\
& \left\{[69.7 \mathrm{pF}-100 \mathrm{pF}]\left[1+\left(1.3 \times 10^{-6}\right)\right]\right\}
\end{aligned}
$$

or

$$
V_{\text {calc }}=4.0199931 \mathrm{kV} \text {. }
$$

$V_{\text {meas }}=4.0200019 \mathrm{kV}$ (see Table 10); therefore $V_{\text {calc }}$ is $2.2 \mathrm{ppm}$ smaller than $v_{\text {meas }}$.

Example 22. $A+2$ ppm error in $\hat{F}_{z}$ at $C\left(z_{L}\right)=100 \mathrm{pF}$ and all errors except the temperature effects.

$$
\begin{aligned}
v_{\text {calc }}^{2} & =-\left(2 \times 10^{4}\right)\left(9.7932024 \times 10^{-3} \mathrm{~N}\right)(2.5 \mathrm{~cm})\left[1+\left(2 \times 10^{-6}\right)\right] / \\
& \left\{[69.7 \mathrm{pF}-100 \mathrm{pF}]\left[1+1.3 \times 10^{-6}\right]\right\}
\end{aligned}
$$

or

$$
V_{\text {calc }}=4.0200013 \mathrm{kV} \text {. }
$$

$V_{\text {meas }}=4.0199939 \mathrm{kV}$; therefore $V_{\text {calc }}$ is $1.8 \mathrm{ppm}$ larger than $V_{\text {meas }}$. 
Example 23. A -2 ppm errors in $\hat{F}_{z}$ at $C\left(z_{L}\right)=69.7 \mathrm{pF}$ and all errors except the temperature effects.

$$
\begin{aligned}
& V_{\text {calc }}=4.0199931 \mathrm{kV} \text { and } \\
& V_{\text {meas }}=4.0200025 \mathrm{kV} ;
\end{aligned}
$$

therefore $V_{\text {calc }}$ is 2.4 smaller than $V_{\text {meas }}$.

Example 24. $A+2 \mathrm{ppm}$ error in $\hat{F}_{z}$ at $C\left(z_{L}\right)=69.7 \mathrm{pF}$ and all errors except the temperature effects.

$$
\begin{aligned}
& V_{\text {calc }}=4.0200013 \mathrm{kV} \text { and } \\
& V_{\text {meas }}=4.0199944 \mathrm{kV} ;
\end{aligned}
$$

therefore $v_{\text {calc }}$ is $1.7 \mathrm{ppm}$ larger than $\mathrm{V}_{\text {meas }}$.

f) Calculations for Type III scans with a brass electrode at $V=9 \mathrm{kV}$.

Example 25. $\theta_{y}$ varies linearly from $0^{\prime \prime}$ to $5^{\prime \prime}$, and $\hat{F}_{z}$ is determined with a $-2 \mathrm{ppm}$ error at $C\left(z_{L}\right)=69.7 \mathrm{pF}$ with all errors except the temperature effects.

A C versus $z_{L}$ plot would have a parabolic shape, but since the maximum change in $\Delta C / C$ is only $+0.3 \mathrm{ppm}$, it can safely be fitted by a straight line for perfect right cylindrical electrodes. 


$$
\begin{aligned}
v_{\text {calc }}^{2}= & -\left(2 \times 10^{4}\right)\left(4.9086 \times 10^{-2} \mathrm{~N}\right)(2.5 \mathrm{~cm})\left[1-\left(2 \times 10^{-6}\right)\right] / \\
& \left\{(69.7 \mathrm{pF})\left[1+\left(0.3 \times 10^{-6}\right)\right]-100 \mathrm{pF}\right\}\left[1+\left(1 \times 10^{-6}\right)\right]
\end{aligned}
$$

or

$\mathrm{V}_{\text {calc }}=8.9999892 \mathrm{kV}$.

$V_{\text {meas }}=9.0000013 \mathrm{kV}$ (see Table 11); therefore $V_{\text {calc }}$ is $1.4 \mathrm{ppm}$ smaller than $V_{\text {meas }}$.

Example 26. $\theta_{y}$ varies linearly from $0^{\prime \prime}$ to $5^{\prime \prime}$, and $\hat{F}_{z}$ is determined with a $+2 \mathrm{ppm}$ error at $C\left(z_{L}\right)=69.7 \mathrm{pF}$ with all errors except the temperature effects.

$$
\begin{aligned}
v_{\text {calc }}^{2}=- & \left(2 \times 10^{4}\right)\left(4.9086 \times 10^{-2} \mathrm{~N}\right)(2.5 \mathrm{~cm})\left[1+\left(2 \times 10^{-6}\right)\right] / \\
& \left\{(69.7 \mathrm{pF})\left[1+\left(0.3 \times 10^{-6}\right)\right]-100 \mathrm{pF}\right\}\left[1+\left(1 \times 10^{-6}\right)\right]
\end{aligned}
$$

or

$$
V_{\text {calc }}=9.0000073 \mathrm{kV}
$$

$V_{\text {meas }}=8.9999833 \mathrm{kV}$; therefore $V_{\text {calc }}$ is $2.6 \mathrm{ppm}$ larger than $V_{\text {meas }}$. 
Example 27. $\theta_{y}$ varies linearly from $5 "$ to 0 " between $z_{L}=0 \mathrm{~cm}$ and $z_{L}=2.5 \mathrm{~cm}$, and $\hat{F}_{z}$ is determined with a -2 ppm error at $c\left(z_{L}\right)=100 \mathrm{pF}$ with all errors except the temperature effects.

$$
\begin{aligned}
v_{\text {calc }}^{2}= & -\left(2 \times 10^{4}\right)\left(4.9086 \times 10^{-2} \mathrm{~N}\right)(2.5 \mathrm{~cm})\left[1-\left(2 \times 10^{-6}\right)\right] / \\
& \left\{69.7 \mathrm{pF}-(100 \mathrm{pF})\left[1+\left(0.3 \times 10^{-6}\right)\right]\right\}\left[1+\left(1 \times 10^{-6}\right)\right]
\end{aligned}
$$

or

$$
V_{\text {calc }}=8.9999817 \mathrm{kV} \text {. }
$$

$V_{\text {meas }}=8.9999878 \mathrm{kV}$; therefore $V_{c a l c}$ is $0.7 \mathrm{ppm}$ smaller thaan $V_{\text {meas }}$.

Example 28. $\theta_{y}$ varies linearly from $5^{\prime \prime}$ to 0 ", and $\hat{\mathrm{F}}_{z}$ is determined with a $+2 \mathrm{ppm}$ error at $C\left(z_{L}\right)=100 \mathrm{pF}$ with all errors except the temperature effects.

$$
\begin{aligned}
v_{\text {calc }}^{2}= & -\left(2 \times 10^{4}\right)\left(4.9086 \times 10^{-2} \mathrm{~N}\right)(2.5 \mathrm{~cm})\left[1+\left(2 \times 10^{-6}\right)\right] / \\
& \left\{69.7 \mathrm{pF}-(100 \mathrm{pF})\left[1+\left(0.3 \times 10^{-6}\right)\right]\right\}\left[1+\left(1 \times 10^{-6}\right)\right]
\end{aligned}
$$

or

$$
V_{\text {calc }}=8.9999998 \mathrm{kV} \text {. }
$$

$V_{\text {meas }}=8.9999698 \mathrm{kV}$; therefore $V_{\text {calc }}$ is $3.3 \mathrm{ppm}$ larger than $V_{\text {meas }}$. 
g) Calculations of Type III scans with an aluminum electrode at $\mathrm{V}=4.02 \mathrm{kV}$.

Example 29. $\theta_{y}$ varies linearly from $0^{\prime \prime}$ to $5^{\prime \prime}$, and $\hat{F}_{z}$ is determined with a -2 ppm error at $C\left(z_{L}\right)=69.7 \mathrm{pF}$ with all errors except the temperature effects.

$$
\begin{aligned}
v_{\text {calc }}^{2}= & -\left(2 \times 10^{4}\right)\left(9.7932024 \times 10^{-3} N\right)(2.5 \mathrm{~cm})\left[1-\left(2 \times 10^{-6}\right)\right] / \\
& \left\{(69.7 \mathrm{pF})\left[1+\left(0.3 \times 10^{-6}\right)\right]-100 \mathrm{pF}\right\}\left[1+\left(1 \times 10^{-6}\right)\right]
\end{aligned}
$$

or

$$
V_{\text {calc }}=4.0199951 \mathrm{kV} \text {. }
$$

$V_{\text {meas }}=4.0200039 \mathrm{kV}$ (see Table 12); therefore $V_{\text {calc }}$ is $2.2 \mathrm{ppm}$ smaller than $V_{\text {meas }}$.

Example 30 . $\theta_{y}$ varies linearly from $0^{\prime \prime}$ to $5^{\prime \prime}$ and $\hat{F}_{z}$ is determined with a +2 ppm error at $C\left(z_{L}\right)=69.7 \mathrm{pF}$ with all errors except the temperature effects.

$$
\begin{aligned}
v_{\text {calc }}^{2}= & -\left(2 \times 10^{4}\right)\left(9.7932024 \times 10^{-3} N\right)(2.5 \mathrm{~cm})\left[1+\left(2 \times 10^{-6}\right)\right] / \\
& \left.\{69.7 \mathrm{pF})\left[1+\left(0.3 \times 10^{-6}\right)\right]-100 \mathrm{pF}\right\}\left[1+\left(1 \times 10^{-6}\right)\right]
\end{aligned}
$$




$$
V_{\text {calc }}=4.0200032 \mathrm{kV} \text {. }
$$

$V_{\text {meas }}=4.0199958 \mathrm{kV}$; therefore $V_{\text {calc }}$ is $1.8 \mathrm{ppm}$ larger than $V_{\text {meas }}$.

Example 31. $\theta_{y}$ varies linearly from $5^{\prime \prime}$ to $0 "$, and $\hat{F}_{z}$ is determined with a $-2 \mathrm{ppm}$ error at $C\left(z_{L}\right)=100 \mathrm{pF}$ with all errors except the temperature effects.

$$
\begin{aligned}
v_{\text {calc }}^{2}= & -\left(2 \times 10^{4}\right)\left(9.7932024 \times 10^{-3} \mathrm{~N}\right)(2.5 \mathrm{~cm})\left[1-\left(2 \times 10^{-6}\right)\right] / \\
& \left\{69.7 \mathrm{pF}-(100 \mathrm{pF})\left[1+\left(0.3 \times 10^{-6}\right)\right]\right\}\left[1+\left(1 \times 10^{-6}\right)\right]
\end{aligned}
$$

or

$$
V_{\text {calc }}=4.0199917 \mathrm{kV} \text {. }
$$

$V_{\text {meas }}=4.0200019 \mathrm{kV}$; therefore $V_{\text {calc }}$ is $2.6 \mathrm{ppm}$ smaller than $V_{\text {meas }}$.

Example 32. $\theta_{y}$ varies linearly from $5^{\prime \prime}$ to 0 ", and $\hat{\mathrm{F}}_{z}$ is determined with a $+2 \mathrm{ppm}$ error at $C\left(z_{L}\right)=100 \mathrm{pF}$ with all errors except the temperature effects.

$$
\begin{aligned}
v_{\text {calc }}^{2}= & -\left(2 \times 10^{4}\right)\left(9.7932024 \times 10^{-3} \mathrm{~N}\right)(2.5 \mathrm{~cm})\left[1+\left(2 \times 10^{-6}\right)\right] / \\
& \left\{69.7 \mathrm{pF}-(100 \mathrm{pF})\left[1+\left(0.3 \times 10^{-6}\right)\right]\right\}\left[1+\left(1 \times 10^{-6}\right)\right]
\end{aligned}
$$

or 


$$
V_{\text {calc }}=4.0199998 \mathrm{kV} \text {. }
$$

$V_{\text {meas }}=4.0199939 \mathrm{kV}$; therefore $V_{\text {calc }}$ is $1.4 \mathrm{ppm}$ larger than $V_{\text {meas }}$. 
DERIVATION OF THE PATH INTEGRAL EQUATION

The change in electrical potential energy, $\Delta U^{e}$, from an initial state $i$ to a state $j$ is

$$
\Delta u^{e}=(1 / 2) c_{j} v_{j}^{2}-(1 / 2) c_{i} v_{i}^{2}=-\int_{l_{i}}^{l_{j}} \vec{F} \cdot d \vec{l}+\int_{q_{j}}^{q_{j}} V d q
$$

[U in $\mathrm{N} \cdot \mathrm{cm} ; \quad C$ in $F ; \quad V$ in $V ; F$ in $N$; $\ell$ in $\mathrm{m} ; \quad q$ in $C]$

where $\vec{F}$ is the electrical force between the electrodes and $q$ is the charge on the electrodes: $q=C V$.

Substituting the derivative of the equation $q=C V(d q=C d V+V d C)$ into Equation U-1 gives

$$
(1 / 2) C_{j} V_{j}^{2}-(1 / 2) C_{i} V_{i}^{2}=-\int_{l_{i}}^{l_{j}} \vec{F} \cdot d \vec{l}+\int_{V_{j}}^{V_{j}} C V d V+\int_{C_{i}}^{C_{j} V^{2} d C}
$$

The last two integrals on the r.h.s. of this equation would be difficult to evaluate unless $V$ were constant. Therefore, assume that $V$ is a constant value $\bar{V}$, and arbitrarily choose this value $\bar{V}$ to be either the average value of $V$ if $V$ varies between states $i$ and $j$ or the nominal voltage. Also, adjust each value of $\vec{F}$ to a new value $\vec{F}^{\prime}$, where $\vec{F}^{\prime}$ is the value that $\vec{F}$ would have been if V were equal to $\vec{V}$. 
(This adjusted value for $F$ will be defined in Appendix V.) Thus, Equation $E-2$ reduces to

$$
(1 / 2) \vec{V}^{2}\left(C_{j}-C_{j}\right)=-\int_{l_{i}}^{l_{j}} \vec{F}^{\prime} \cdot d \vec{l}+\vec{v}^{2}\left(C_{j}-C_{j}\right)
$$

or

$$
\int_{\ell_{j}}^{\ell_{j}} \vec{F}^{\prime} \cdot d \vec{l}=(1 / 2) \vec{V}^{2}\left(c_{j}-C_{i}\right)
$$

Using the relative coordinate system of Figure 7, Equation E-3 becomes

$$
\int_{h_{x_{i}}}^{h_{x}}{ }_{j} F_{h_{x}}^{\prime} d h_{x}+\int_{h_{y_{i}}}^{h_{y_{j}}} 2 F_{h_{y}^{\prime}} d h_{y}+\int_{z_{L_{i}}}^{z_{L}}{ }_{j} F_{z_{L}^{\prime}} d z_{L}=(1 / 2) \bar{v}^{2}\left(c_{j}-C_{j}\right)
$$

$\left[F^{\prime}\right.$ in $N ; h, z_{L}$ in $m$; $V$ in $V ; C$ in $\left.F\right]$.

So, the mechanical work, $W^{e}=\int \vec{F}^{\prime} \cdot d \vec{l}$, to move the system from point $\left(h_{x_{i}}, h_{y_{j}}, z_{L_{j}}\right)$ to point $\left(h_{x_{j}}, h_{y_{j}}, z_{L_{j}}\right)$ is equal to the change in electrical energy stored in the field. This work and energy is expressed in $\mathrm{N} \cdot \mathrm{m}$ in Equation $\mathrm{U}-4$. It is more convenient to express these terms in $\mathrm{N} \cdot \mathrm{cm}$, and to use multiples and submultiples of $h, v$ and $C$. In equilibrium, Equation U-4 then becomes

$$
w^{e}=\left(2 \times 10^{-4}\right) \int_{h_{x_{j}}}^{h^{x}} \hat{F}_{h_{x}} d h_{x}+\left(2 \times 10^{-4}\right) \int_{h_{y_{j}}}^{h_{y}} \hat{F}_{h_{y}^{\prime}} d h_{y}
$$




$$
+\int_{z_{L_{i}}}^{z_{L_{j}}} \hat{F}_{z_{L}}^{\prime} d z_{L}=(1 / 2)\left(1 \times 10^{-4}\right) \bar{V}^{2}\left(\hat{C}_{j}-\hat{C}_{i}\right)
$$

[W $W^{e}$ in $N \cdot c m ; F^{\prime}$ in $N$; $h$ in $\mu \mathrm{m} ; Z_{L}$ in $\mathrm{cm}$; $\bar{V}$ in $k V ; C$ in $\left.p F\right]$.

Note in Figure 7 that $\hat{F}_{z}^{\prime}$ is actually negative, i.e. its direction is opposite to that for $z_{L}$. 


\section{APPENDIX $V$}

THE ADJUSTMENT OF $F$ TO $F^{\prime}$ IN THE PATH INTEGRAL

The partial derivatives of Equation U-4 are

$$
\begin{aligned}
& F_{h_{x}}^{\prime}=(1 / 4) \bar{V}^{2}\left[\partial C\left(h, z_{L}\right) / \partial h_{x}\right]_{h_{y}, z_{L}} \\
& F_{h_{y}}^{\prime}=(1 / 4) \bar{V}^{2}\left[\partial c\left(h, z_{L}\right) / \partial h_{y}\right]_{h_{x}, z_{L}} \\
& F_{z_{L}}^{\prime}=(1 / 2) \bar{V}^{2}\left[\partial c\left(h, z_{L}\right) / \partial z_{L}\right]_{h_{x}}, h_{y}
\end{aligned}
$$

[F in $N$; $V$ in $V ; C$ in $p F ; h, z_{L}$ in $m$ ].

It is clear when comparing Equations $V-1$ to $V-3$ with Equations $E-3$ to $E-5$ that

$$
\vec{F}^{\prime}\left(h, z_{L}\right)=\left[\vec{V}^{2} / V^{2}\left(h, z_{L}\right)\right] \vec{F}\left(h, z_{L}\right)=\xi\left(h, z_{L}\right) \vec{F}\left(h, z_{L}\right)
$$

$\left[F^{\prime}, F\right.$ in $N ; \bar{V}, V$ in $k V ; h$ in $\mu m ; z_{L}$ in $\left.\mathrm{cm}\right]$.

where $\xi=\vec{V}^{2} / V^{2}$ is the factor by which $\vec{F}$ is adjusted to a new value $\vec{F}^{\prime}$, such that $\vec{F}^{\prime}$ is the value that $\vec{F}$ would have been if $V$ vere equal to $\bar{v}$. Equation $V-4$ holds for each component of $\vec{F}$, i.e. for $F_{h_{x}}$, $F_{h_{y}}$ and $F_{z_{L}}$. 
A CHECK THAT THE PATH INTEGRAL IS CONSERVATIVE

The path integral must be conservative. If the integral is conservative then it should not matter which path is taken between the initial state $i$ and the final state $j$ shown in Figure 21 . To simplify the calculations, the two paths indicated in the figure are selected.

Note that the values of $\hat{h}_{y}$ for these two paths are for those at high voltages not for those at low voltages. Therefore, in that part of the path used to calculate $w_{4}^{e}, d_{y}$ and/or $\theta_{y}$ are not constant, but instead vary in such a manner that $h_{y}$ is constant. Table 9 shows that this would not be the case for a Type II scan with $d_{y}=0$ and $\theta_{y}=$ constant because for that case, $\hat{h}_{y}$ varies at high voltages.

If Equation 4-3 is conservative then

$$
w_{1}^{e}+w_{2}^{e}=w_{3}^{e}+w_{4}^{e}
$$

or

$$
\begin{aligned}
& -\left(6.06 \times 10^{-4}\right) \bar{V}_{1+2^{2} L_{f}}^{2}+\left(6.5 \times 10^{-13}\right) \bar{V}_{1+2}^{2} C\left(z_{L_{f}}\right)\left(\hat{h}_{y}^{2} / 2\right)= \\
& \left(6.5 \times 10^{-13}\right) \bar{V}_{3+4}^{2} C(0)\left(\hat{h}_{y}^{2} / 2\right)-\left(6.06 \times 10^{-4}\right) \bar{V}_{3+4}^{2}\left[1+\left(6.5 \times 10^{-9}\right) \hat{h}_{y}^{2}\right] z_{L_{f}}
\end{aligned}
$$


If $\bar{V}_{1+2}=\bar{V}_{3+4}$, then this equation becomes

$$
C\left(z_{L_{f}}\right)=C(0)-(12.12) z_{L_{f}}
$$

or

$$
C\left(z_{L_{f}}\right)=\left[100 p F-(12.12 p F / c m) z_{L_{f}}\right]
$$

This agrees with Equation 2-13, and therefore the path integral is conservative - but only if the normalization values $\bar{V}_{1+2}$ and $\bar{V}_{3+4}$ are chosen to be the same for each path. This normalization value is completely arbitrary (as shown in Example 2 of Appendix $Y$ ), so it might just as well be the nominal value of the voltage applied to the electrodes, i.e. $\bar{V}=V_{N}$, and $V_{N}=9 k V$ for $m=5$ grams, or $V_{N}=4.02 k V$ for $\mathrm{m}=1$ gram.

The other necessary condition for the path integral is that the mechanical work required to translate and/or rotate the electrodes must equal the change in electrical energy stored in the field between the electrodes, i.e.

$$
w_{1}^{e}+w_{2}^{e}=(1 / 2)\left(1 \times 10^{-4}\right) V_{N}^{2}\left[\hat{c}_{f}-\hat{c}_{j}\right]
$$

or

$$
-\left(6.06 \times 10^{-4}\right) V_{N}^{2} L_{f}+\left(6.5 \times 10^{-13}\right) V_{N}^{2} C\left(z_{L_{f}}\right)\left(\hat{h}_{y}^{2} / 2\right)=
$$




$$
(1 / 2)\left(1 \times 10^{-4}\right) V_{N}^{2}\left\{C\left(z_{L_{f}}\right)\left[1+\left(6.5 \times 10^{-9}\right) \hat{h}_{y}^{2}\right]-c(0)\right\}
$$

or

$$
\begin{aligned}
& C\left(z_{L_{f}}\right)=C(0)-(12.12) z_{L_{f}} \\
& C\left(z_{L_{f}}\right)=\left[100 p F-(12.12 p F / c m) z_{L_{f}}\right] .
\end{aligned}
$$

This again agrees with Equation 2-13. 
THE UNKNOWN PART OF THE PATH INTEGRAL

The horizontal integrals $\int 2 \hat{F}_{h_{x}}^{\prime} d h_{x}$ and $\int 2 \hat{F}_{h_{y}^{\prime}}^{\prime} d h_{y}$ are unknown in Equation 4-4 because $\hat{F}_{h_{x_{A}}^{\prime}}$ and $\hat{F}_{h_{y}}^{\prime}$ cannot be measured with the balance, and measurements of the $\hat{d}_{x}$ and $\hat{d}_{y}$ contributions to $\hat{h}_{x}$ and $\hat{h}_{y}$ would require expensive laser inteferometry techniques.

This appendix will determine the size of these missing integrals for the two paths shown in Figure 21 with a brass suspended electrode at $V_{N}=9 \mathrm{kV}$. Let $\mathrm{C}(0)=100 \mathrm{pF}$ and $\mathrm{C}\left(\mathrm{z}_{\mathrm{L}_{f}}\right)=\mathrm{C}(2.5 \mathrm{~cm})=69.7 \mathrm{pF}$. Also, let $d_{y}=0 \mu \mathrm{m}$ and $\theta_{y}=5^{\prime \prime}$ at point $\left(0, \hat{h}_{y}, 0\right)$; so $\hat{\gamma}_{y}=6.80$ " and $\hat{h}_{y}=26.8 \mu \mathrm{m}$ at that point. Remember that $\hat{h}_{y}$ is constant for the $w_{4}^{e}$ integral in this figure; therefore, $\theta_{y}=8.35^{\prime \prime}, \hat{\gamma}_{y}=4.84^{\prime \prime}$ and $\hat{h}_{y}=26.8 \mu \mathrm{m}$ at point $(0$, $\left.\hat{h}_{y}, z_{L_{f}}\right)$.

Equations $4-3$ and $V-4$ yield the results

$$
\begin{aligned}
w_{2}^{e} / w_{1}^{e} & =\left(6.5 \times 10^{-13}\right) V_{N}^{2}(69.7 p F)(1 / 2)(26.8 \mu \mathrm{m})^{2} / \\
& {\left[-\left(6.06 \times 10^{-4}\right) V_{N}^{2}(2.5 \mathrm{~cm})\right] } \\
w_{2}^{e} / w_{1}^{e} & =-1.07 \times 10^{-5}
\end{aligned}
$$

or the path integral should be $10.7 \mathrm{ppm}$ less negative than the $W_{1}^{e}$ integral for the first path. For the second path 


$$
\begin{aligned}
W_{3}^{e} / W_{4}^{e}= & \left(6.5 \times 10^{-13}\right) V_{N}^{2}(100 \mathrm{pF})(1 / 2)(26.8 \mu \mathrm{m})^{2} / \\
& \left\{-\left(6.06 \times 10^{-4}\right) V_{N}^{2}\left[1+\left(6.5 \times 10^{-9}\right)(26.8 \mu \mathrm{m})^{2}\right](2.5 \mathrm{~cm})\right\} \\
W_{3}^{e} / w_{4}^{e}= & -1.54 \times 10^{-5}
\end{aligned}
$$

or the path integral is actually $15.4 \mathrm{ppm}$ less negative than the $W_{4}^{e}$ integral.

This error can therefore be quite large. Its magnitude depends upon the particular path taken during a scan, and it will have the same sign,independent of the carriage translation direction (i.e. the absolute value of the path integral is actually less than $\left.\left|\int \hat{F}_{z_{L}} d z_{L}\right|\right)$ 


\section{APPENDIX Y}

ERROR CALCULATIONS FOR THE PATH INTEGRAL METHOD

a) Method used to estimate the voltage ratio uncertainty.

The voltage ratio, $V_{c a l c} / V_{N}$, which can be obtained from Equation $4-4$, is

$$
v_{\text {calc }} / V_{\text {meas }}=\left\{\left[-\left(2 \times 10^{4}\right) \int 1 / v_{\text {meas }}^{2}\left|\hat{F}_{z_{L}}\right| d_{z_{L}}+4 \int 1 / V_{\text {meas }}^{2} \hat{F}_{h} d h\right] /\left(\hat{C}_{f}-\hat{C}_{j}\right)\right\}^{\frac{2}{2}}(Y-1)
$$

$\left[F_{z_{L}}, F_{h}\right.$ in $N ; V_{\text {calc }}, V_{N}, V_{\text {meas }}$ in $k V ; C$ in $p F ; h$ in $\mu m ; z_{L}$ in $\mathrm{cm}]$.

The quantities in Equation $Y-1$ that have errors associated with them are $V_{\text {meas }}, \hat{F}_{z_{L}}, z_{L}, \hat{F}_{h}, \hat{C}_{i}$ and $\hat{C}_{f}$. The horizontal component of the path integral is experimentally unknown - so it can be treated as an uncertainty, rather than $\hat{F}_{h}$ and $h$ individually. Also, the errors involved in measuring the capacitance at low voltage rather than at the high voltages must be included in $\hat{C}_{i}$ and $\hat{C}_{f}$ (along with the dynamical measurement errors and any errors resulting from temperature fluctuations.) For example, if the carriage tilts linearly from $\theta=0$ " to $5^{\prime \prime}$ between $C=100 \mathrm{pF}$ and $69.7 \mathrm{pF}$ for a brass suspended electrode at $V_{N}=9 \mathrm{kV}$, then the path integral is too large by $4 \mathrm{ppm}$ when the horizontal component is not included, and $\hat{C}_{f}$ is 1.4 ppm too small because of the low voltage measurement problem. If $\theta_{y}$ 
varies from $5^{\prime \prime}$ to 0 " between $\mathrm{C}=100 \mathrm{pF}$ and $69.7 \mathrm{pF}$ then the path integral is $16 \mathrm{ppm}$ too small, and $\hat{C}_{\dot{i}}$ is $4.4 \mathrm{ppm}$ too small. (Notice that the horizontal component of the path integral and the low voltage capacitance errors are correlated.)

There is a random error in determining $m$, which (according to Equation $\mathrm{P}-18$ ) should be included in the uncertainty of $\hat{F}_{z_{L}}$ - along with the systematic static and dynamic errors. The static systematic error is also correlated with the low-voltage error in measuring $\hat{C}$ and with the horizontal component of the path integral. Therefore, the uncertainties of Equation $\mathrm{Y}-1$ are correlated mixtures of random and systematic errors. Rather than attempting to assign correlation coefficients and deciding how to combine random and systematic errors for nonexistent data, only measured quantities and their assumed measurement uncertainties will be used. The voltage ratio will be calculated for a variety of carriage scans, using the low voltage capacitance values and neglecting the horizontal path integral components. The correlation effects and the effects due to the unmeasurable quantities should thereby be reflected in this uncertainty estimate.

b) Calculations for Type I scans with a brass electrode at $V_{N}=9 \mathrm{kV}$.

Example 1. Perfectly aligned right circular cylinders with no errors. 
Let mass $m$ be such that $\hat{F}_{z}=m g$ at $V_{N}=9 \mathrm{kV}$ when $\hat{h}=0$. Since $\hat{h}=0$, Equation 4-2 becomes

$$
\hat{F}_{z}=m g=\left(6.06 \times 10^{-4}\right)(9 \mathrm{kV})^{2}=4.9086 \times 10^{-2} \mathrm{~N}
$$

and $V_{\text {meas }}=V_{N}=9 \mathrm{kV}$. Also, $\left|\hat{F}_{Z}\right|=\left|\hat{F}_{z_{L}}\right|$ and $\left|\hat{F}_{Z_{L}}^{\prime}\right|=\mid \hat{F}_{Z}$ because $\xi\left(\hat{h}_{,} z_{L}\right)=1$.

Therefore, Equation 4-4 becomes

$$
\begin{aligned}
v_{\text {calc }}^{2}= & -\left(2 \times 10^{4}\right) \mathrm{mg}\left(\Delta \mathrm{z}_{\mathrm{L}} / \Delta \mathrm{C}\right)=-\left(2 \times 10^{4}\right)\left(4.9086 \times 10^{-2} \mathrm{~N}\right)(2.5 \mathrm{~cm}) / \\
& (69.7 \mathrm{pF}-100 \mathrm{pF})
\end{aligned}
$$

or $V_{\text {calc }}=9 \mathrm{kV}=V_{\text {meas }}$. Therefore, there is no error in this example.

Example 2. A -2 ppm error in $\hat{F}_{z}$ and a possible combination of other errors.

The static and dynamic errors are such that $\hat{F}_{z}$ appears to be 2 ppm smaller than $m g$ when $\hat{F}_{z}$ actually equals $\mathrm{mg}$. $\hat{\mathrm{F}}_{z}$ would thus be increased by 2 ppm until $\hat{\beta}_{y}^{(O N)}=\hat{\beta}_{y}^{(O F F)}$, so that

$$
V_{\text {meas }}^{2}=\left(4.9086 \times 10^{-2} N\right)\left[1+\left(2 \times 10^{-6}\right)\right] /\left(6.06 \times 10^{-4}\right)
$$

or

$$
V_{\text {meas }}=9.000009 \mathrm{kV} \text {. }
$$




$$
\begin{aligned}
v_{\text {calc }}^{2}=-\left(2 \times 10^{4}\right)(\mathrm{mg}) V_{N}^{2} / V_{\text {meas }}^{2}\left(\Delta z_{L} / \Delta C\right)=-\left(2 \times 10^{4}\right)(\mathrm{mg})\left[1-\left(2 \times 10^{-6}\right)\right]\left(\Delta z_{L} / \Delta C\right) \\
V_{\text {calc }}^{2}=-\left(2 \times 10^{4}\right)\left(4.9086 \times 10^{-2} N\right)\left[1-\left(2 \times 10^{-6}\right)\right](2.5 \mathrm{~cm})\left[1-\left(2 \times 10^{-6}\right)\right] / \\
\quad\left\{69.7 \mathrm{pF}-(100 \mathrm{pF})\left[1+\left(1 \times 10^{-6}\right)\right]\right\}
\end{aligned}
$$

or

$V_{\text {calc }}=8.9999668 \mathrm{kV}$.

Therefore, $V_{\text {calc }}$ is $3.7 \mathrm{ppm}$ smaller than $V_{N}$.

Rather than using $V_{N}$ as the normalization value, let us use $\bar{V}=V_{\text {meas }}=9.000009 \mathrm{kV}$.

$$
\begin{aligned}
v_{\text {calc }}^{2} & =-\left(2 \times 10^{4}\right)(\mathrm{mg}) \bar{V}^{2} / \vec{V}_{\text {meas }}^{2}\left(\Delta z_{L} / \Delta C\right)=-\left(2 \times 10^{4}\right)(\mathrm{mg})\left(\Delta z_{\mathrm{L}} / \Delta \mathrm{C}\right) \\
v_{\text {calc }}^{2} & =-\left(2 \times 10^{4}\right)\left(4.9086 \times 10^{-2} \mathrm{~N}\right)(2.5 \mathrm{~cm})\left[1-\left(2 \times 10^{-6}\right)\right] / \\
& \left\{69.7 \mathrm{pF}-(100 \mathrm{pF})\left[1+\left(1 \times 10^{-6}\right)\right]\right\}
\end{aligned}
$$

or

$$
\mathrm{V}_{\text {calc }}=8.9999758 \mathrm{kV} \text {. }
$$

Therefore $V_{\text {calc }}$ is $3.7 \mathrm{ppm}$ smaller than $\bar{V}$, which is the same result as that for a nominal voltage $\left(V_{N}=9 \mathrm{kV}\right)$ normalization factor. The normalization factor is completely arbitrary as long as it is used 
consistently and correctly. The nominal voltage will be used as the normalization value in the examples.

Example $3 . A+2$ ppm error in $\hat{F}_{z}$ and a possible combination of other errors.

$$
\begin{aligned}
& v_{N}^{2} / v_{\text {meas }}^{2}=1+\left(2 \times 10^{-6}\right) \\
& v_{\text {calc }}^{2}=-\left(2 \times 10^{4}\right)\left(4.9086 \times 10^{-2} \mathrm{~N}\right)\left[1+\left(2 \times 10^{-6}\right)\right](2.5 \mathrm{~cm})\left[1+\left(2 \times 10^{-6}\right)\right] / \\
& \quad\left\{(69.7 \mathrm{pF})\left[1+\left(1 \times 10^{-6}\right)\right]-100 \mathrm{pF}\right\}
\end{aligned}
$$

or

$$
V_{\text {calc }}=9.0000267 \mathrm{kV} \text {. }
$$

Therefore, $V_{\text {calc }}$ is $2.9 \mathrm{ppm}$ larger than $v_{N}$. Note that if a $-1 \mathrm{ppm}$ error is assumed for $100 \mathrm{pF}$ rather than the $+1 \mathrm{ppm}$ error for $69.7 \mathrm{pF}$, then $v_{\text {calc }}$ would be $3.7 \mathrm{ppm}$ larger than $\mathrm{V}_{\mathrm{N}}$. The $+1 \mathrm{ppm}$ error is chosen because this is the sign for a dynamical capacitance systematic measurement error.

Example 4. A -2 ppm error in $\hat{F}_{z}$ and a maximum combination of other errors.

$$
v_{\text {calc }}^{2}=-\left(2 \times 10^{4}\right)\left(4.9086 \times 10^{-2} \mathrm{~N}\right)\left[1-\left(2 \times 10^{-6}\right)\right](2.5 \mathrm{~cm})\left[1-\left(2 \times 10^{-6}\right)\right] /
$$




\section{$\left\{(69.7 \mathrm{pF})\left[1-\left(1 \times 10^{-6}\right)\right]-(100 \mathrm{pF})\left[1+\left(2 \times 10^{-6}\right)\right]\right\}$}

or

$$
\mathrm{V}_{\mathrm{calc}}=8.9999507 \mathrm{kV} \text {. }
$$

Therefore, $V_{\text {calc }}$ is $5.5 \mathrm{ppm}$ smaller than $V_{\mathrm{N}}$.

Example 5 . A $+2 \mathrm{ppm}$ error in $\hat{\mathrm{F}}_{\mathrm{z}}$ and a maximum combination of other errors.

$$
\begin{aligned}
& v_{\text {calc }}^{2}=-\left(2 \times 10^{4}\right)\left(4.9086 \times 10^{-2} \mathrm{~N}\right)\left[1+\left(2 \times 10^{-6}\right)\right](2.5 \mathrm{~cm})\left[1+\left(2 \times 10^{-6}\right)\right] / \\
& \left\{(69.7 \mathrm{pF})\left[1+\left(2 \times 10^{-6}\right)\right]-(100 \mathrm{pF})\left[1-\left(1 \times 10^{-6}\right)\right]\right\}
\end{aligned}
$$

or

$$
V_{\text {calc }}=9.0000443 \mathrm{kV} \text {. }
$$

Therefore, $V_{\text {calc }}$ is $4.9 \mathrm{ppm}$ larger than $V_{\mathrm{N}}$.

Examples 4 and 5 are very pessimistic. Examples 2 and 3 provide a better estimate of the uncertainty involved in using the path integral method for a Type I scan - which is $+3 \mathrm{ppm}$ and $-3.5 \mathrm{ppm}$, or a $\pm 3 \mathrm{ppm}$ measurement. 
c) Calculation for a Type I scan with an aluminum electrode at $V_{N}=4.02$ kV.

Example 6. A -2 ppm error in $\hat{F}_{z}$ and a possible combination of other errors.

Let mass $m$ be such that $\hat{F}_{z}=m g$ at $V_{N}=4.02 k V$ when $\hat{h}=0$. Since $\hat{h}=0$, Equation 4-2 becomes

$$
\hat{F}_{z}=m g=\left(6.06 \times 10^{-4}\right)(4.02 \mathrm{kV})^{2}=9.7932024 \times 10^{3} \mathrm{~N}
$$

$\hat{F}_{z}$ appears to be $2 \mathrm{ppm}$ smaller than this value for $\mathrm{mg}$; therefore, the voltage is adjusted until

$$
V_{\text {meas }}^{2}=\left(9.7932024 \times 10^{-3} N\right)\left[1+\left(2 \times 10^{-6}\right)\right] /\left(6.06 \times 10^{-4}\right)
$$

or

$$
\begin{aligned}
& v_{\text {meas }}=4.020004 \mathrm{kV} . \\
& v_{\text {calc }}^{2}=-\left(2 \times 10^{4}\right)(\mathrm{mg})\left(v_{N}^{2} / v_{\text {meas }}^{2}\right)\left(\Delta z_{L} / \Delta C\right)=-\left(2 \times 10^{4}\right)(\mathrm{mg})\left[1-\left(2 \times 10^{-6}\right)\right]\left(\Delta z_{L} / \Delta C\right) \\
& v_{\text {calc }}^{2}=-\left(2 \times 10^{4}\right)\left(9.7932024 \times 10^{-3} \mathrm{~N}\right)\left[1-\left(2 \times 10^{-6}\right)\right](2.5 \mathrm{~cm})\left[1-\left(2 \times 10^{-6}\right)\right] / \\
& \quad\left\{69.7 \mathrm{pF}-(100 \mathrm{pF})\left[1+\left(1 \times 10^{-6}\right)\right]\right\}
\end{aligned}
$$




$$
V_{\text {calc }}=4.0199851 \mathrm{kV} \text {. }
$$

Therefore, $V_{c a l c}$ is $3.7 \mathrm{ppm}$ smaller than $V_{N}$.

This example agrees with Example 2 for the brass suspended electrode, at $V_{N}=9 \mathrm{kV}$, as expected, because it should not matter what electrode is used, at what voltage, as long as the carriage is translated along the combined electrode symmetry axis.

d) Calculations for Type II scans with a brass electrode at $V_{N}=9 \mathrm{kV}$ and $\theta_{y}=5^{\prime \prime}$

Example 7. No measurement errors involved in the experiment.

Figure 22 shows a plot of $\delta \hat{F}^{\prime} /(m g)$ versus $z_{L}$, where $\delta \hat{F}^{\prime} /(m g)$ is derived from the $V_{\text {meas }}$ values 1 isted in column 7 of Table 9. The straight 1 ine at $\delta \hat{F}^{\prime} /(\mathrm{mg})=+2.8 \mathrm{ppm}$ yields the same change in path integral as the curved 1 ine. Therefore, $\int \hat{F}^{\prime} d z_{L}=(m g)\left[1+\left(2.8 \times 10^{-6}\right)\right] \Delta z_{L}$. Example 13 of Appendix $T$ shows that the capacitance is $+0.3 \mathrm{ppm}$ larger than the $C\left(z_{L}\right)$ value at every point $z_{L}$.

$$
\begin{array}{r}
V_{\text {calc }}^{2}=-\left(2 \times 10^{4}\right)\left(4.9086 \times 10^{-2} \mathrm{~N}\right)\left[1+\left(2.8 \times 10^{-6}\right)\right](2.5 \mathrm{~cm}) / \\
\left\{(69.7 \mathrm{pF})\left[1+\left(0.3 \times 10^{-6}\right)\right]-(100 \mathrm{pF})\left[1+\left(0.3 \times 10^{-6}\right)\right]\right\}
\end{array}
$$

or

$$
V_{\text {calc }}=9.0000109 \mathrm{kV} \text {. }
$$


Therefore, $V_{\text {calc }}$ is $1.2 \mathrm{ppm}$ larger than $V_{N}$ with no experimental errors present. This discrepancy arises because the horizontal parts of the path integral are unknown, and because the force is measured at $9 \mathrm{kV}$ where the suspended electrode has swung out to some equilibrium angle (causing a $1.7 \mathrm{ppm}$ to $4.7 \mathrm{ppm}$ increase in the actual capacitance, depending upon the value of $z_{L}$ ), but the capacitance is measured at $V_{r m s}=100 \mathrm{~V}$ (resulting in only a $0.3 \mathrm{ppm}$ capacitance increase).

Example 8. A -2 ppm error in $\hat{F}_{z}$ and a possible combination of other errors.

$$
\begin{aligned}
V_{\text {calc }}^{2}= & -\left(2 \times 10^{4}\right)\left(4.9086 \times 10^{-2} \mathrm{~N}\right)\left[1+\left(0.8 \times 10^{-6}\right)\right](2.5 \mathrm{~cm})\left[1-\left(2 \times 10^{-6}\right)\right] / \\
& \left\{(69.7 \mathrm{pF})\left[1+\left(0.3 \times 10^{-6}\right)\right]-(100 \mathrm{pF})\left[1+\left(1.3 \times 10^{-6}\right)\right]\right\}
\end{aligned}
$$

or

$$
V_{\text {calc }}=8.9999811 \mathrm{kV} \text {. }
$$

Therefore $V_{\text {calc }}$ is $2.1 \mathrm{ppm}$ smaller than $V_{N}$.

Example 9. A +2 ppm error in $\hat{F}_{z}$ and a possible combination of other errors.

$$
\begin{gathered}
v_{\text {calc }}^{2}=-\left(2 \times 10^{4}\right)\left(4.9086 \times 10^{-2} \mathrm{~N}\right)\left[1+\left(4.8 \times 10^{-6}\right)\right](2.5 \mathrm{~cm})\left[1+\left(2 \times 10^{-6}\right)\right] / \\
\left\{(69.7 \mathrm{pF})\left[1+\left(1.3 \times 10^{-6}\right)\right]-(100 \mathrm{pF})\left[1+\left(0.3 \times 10^{-6}\right)\right]\right\}
\end{gathered}
$$

or 


$$
V_{\text {calc }}=9.0000393 \mathrm{kV} \text {. }
$$

Therefore, $V_{\text {calc }}$ is $4.3 \mathrm{ppm}$ larger than $V_{\mathrm{N}}$.

e) Calculations for Type II scans with an aluminum electrode at $V_{N}=4.02 \mathrm{kV}$ and $\theta=5^{\prime \prime}$.

Example 10. No measurement errors involved in the experiment.

A plot of the $\delta \hat{F}^{\prime} / \mathrm{mg}$ values obtained using column 7 of Table 10 versus $z_{L}$ yields a straight line at $\delta \hat{F}^{\prime} / m g=+0.8 \mathrm{ppm}$.

$$
\begin{gathered}
v_{\text {calc }}^{2}=-\left(2 \times 10^{4}\right)\left(9.7932024 \times 10^{-3} N\right)\left[1+\left(0.8 \times 10^{-6}\right)\right](2.5 \mathrm{~cm}) / \\
\left\{(69.7 \mathrm{pF})\left[1+\left(0.3 \times 10^{-6}\right)\right]-(100 \mathrm{pF})\left[1+\left(0.3 \times 10^{-6}\right)\right]\right\}
\end{gathered}
$$

or

$$
V_{\text {calc }}=4.0200008 \mathrm{kV} \text {. }
$$

Therefore, $V_{\text {calc }}$ is $0.1 \mathrm{ppm}$ larger than $V_{N}$ with no experimental errors present.

Example 11. A -2 ppm error in $\hat{F}_{z}$ and a possible combination of other errors.

$$
\begin{aligned}
V_{\text {calc }}^{2} & =-\left(2 \times 10^{4}\right)\left(9.7932024 \times 10^{-3} N\right)\left[1-\left(1.2 \times 10^{-6}\right)\right](2.5 \mathrm{~cm})\left[1-\left(2 \times 10^{-6}\right)\right] / \\
& \left\{(69.7 \mathrm{pF})\left[1+\left(0.3 \times 10^{-6}\right)\right]-(100 \mathrm{pF})\left[1+\left(1.3 \times 10^{-6}\right)\right]\right\}
\end{aligned}
$$


or

$$
V_{\text {calc }}=4.0199861 \mathrm{kV} \text {. }
$$

Therefore, $v_{\text {calc }}$ is $3.5 \mathrm{ppm}$ smaller than $V_{N}$.

Example 12. A $A$ ppm error in $\hat{F}_{z}$ and a possible combination of other errors.

$$
\begin{aligned}
& v_{\text {calc }}^{2}=-\left(2 \times 10^{4}\right)\left(9.7932024 \times 10^{-3} N\right)\left[1+\left(2.8 \times 10^{-6}\right)\right](2.5 \mathrm{~cm})\left[1+\left(2 \times 10^{-6}\right)\right] / \\
&\left.\left\{(69.7 \mathrm{pF})\left[1+\left(1.3 \times 10^{-6}\right)\right]-(100 \mathrm{pF})\left[1+0.3 \times 10^{-6}\right)\right]\right\}
\end{aligned}
$$

or

$$
V_{\text {calc }}=4.0200135 \mathrm{kV} \text {. }
$$

Therefore, $V_{\text {calc }}$ is $3.3 \mathrm{ppm}$ larger than $V_{\mathrm{N}}$.

f) Calculations for Type III scans with a brass electrode at $V_{N}=9 \mathrm{kV}$.

Example 13. $\theta_{y}$ varies linearly from $5^{\prime \prime}$ to $0^{\prime \prime} ; \hat{F}_{z}$ is determined with a -2 ppm error, and a possible combination of other errors.

A plot of the $\delta \hat{F}^{1} / m g$ values obtained using column 7 of Table 11 versus $z_{L}$ yields a straight line at $\delta \hat{F}^{\prime} /(\mathrm{mg})=+1.4 \mathrm{ppm}$.

$$
\begin{aligned}
V_{\text {calc }}^{2}=-\left(2 \times 10^{4}\right)\left(4.9086 \times 10^{-2} \mathrm{~N}\right)\left[1-\left(0.6 \times 10^{-6}\right)\right](2.5 \mathrm{~cm})\left[1-\left(2 \times 10^{-6}\right)\right] / \\
\left\{69.7 \mathrm{pF}-(100 \mathrm{pF})\left[1+\left(1.3 \times 10^{-6}\right)\right]\right\}
\end{aligned}
$$




$$
V_{\text {calc }}=8.9999687 \mathrm{kV} \text {. }
$$

Therefore, $V_{\text {calc }}$ is $3.5 \mathrm{ppm}$ smaller than $V_{N}$.

Example 14. $\theta_{y}$ varies linearly from $5^{\prime \prime}$ to 0 "; $\hat{F}_{z}$ is determined with a $+2 \mathrm{ppm}$ error, and a possible combination of other errors.

$$
\begin{aligned}
& V_{\text {calc }}^{2}=-\left(2 \times 10^{4}\right)\left(4.9086 \times 10^{-2} \mathrm{~N}\right)\left[1+\left(3.4 \times 10^{-6}\right)\right](2.5 \mathrm{~cm})\left[1+\left(2 \times 10^{-6}\right)\right] / \\
&\left\{(69.7 \mathrm{pF})\left[1+\left(1 \times 10^{-6}\right)\right]-(100 \mathrm{pF})\left[1+\left(0.3 \times 10^{-6}\right)\right]\right\}
\end{aligned}
$$

or

$$
V_{\text {calc }}=9.0000286 \mathrm{kV} \text {. }
$$

Therefore, $V_{\text {calc }}$ is $3.1 \mathrm{ppm}$ larger than $V_{\mathrm{N}}$.

g) Calculations for Type III scans with an aluminum electrode at $V_{N}=4.02 \mathrm{kV}$.

Example $15 . \quad \theta_{y}$ varies linearly from $5^{\prime \prime}$ to $0^{\prime \prime} ; \hat{F}_{z}$ is determined with a -2 ppm error, and a possible combination of other errors. A plot of the $\delta \hat{F}^{\prime} /(\mathrm{mg})$ values obtained using column 7 of Table 12 versus $z_{L}$ yields a straight line at $\delta \hat{F}^{\prime} /(\mathrm{mg})=+0.3 \mathrm{ppm}$.

$$
v_{\text {calc }}^{2}=-\left(2 \times 10^{4}\right)\left(9.7932024 \times 10^{-3} N\right)\left[1-\left(1.7 \times 10^{-6}\right)\right](2.5 \mathrm{~cm})\left[1-\left(2 \times 10^{-6}\right)\right] /
$$




$$
\left\{69.7 \mathrm{pF}-(100 \mathrm{pF})\left[1+\left(1.3 \times 10^{-6}\right]\right\}\right.
$$

or

$$
V_{\text {calc }}=4.0199838 \mathrm{kV} \text {. }
$$

Therefore, $V_{\text {calc }}$ is $4.1 \mathrm{ppm}$ smaller than $V_{N}$.

Example 16. $\theta_{y}$ varies linearly from $5^{\prime \prime}$ to $0 " ; \hat{F}_{z}$ is determined with a +2 ppm error, and a possible combination of other errors.

$$
\begin{aligned}
V_{\text {ca Ic }}^{2} & =-\left(2 \times 10^{4}\right)\left(9.7932024 \times 10^{-3} N\right)\left[1+\left(2.3 \times 10^{-6}\right)\right](2.5 \mathrm{~cm})\left[1+\left(2 \times 10^{-6}\right)\right] / \\
& \left\{(69.7 \mathrm{pF})\left[1+\left(1 \times 10^{-6}\right)\right]-(100 \mathrm{pF})\left[1+\left(0.3 \times 10^{-6}\right)\right]\right\}
\end{aligned}
$$

or

$$
V_{\text {calc }}=4.0200105 \mathrm{kV} \text {. }
$$

Therefore, $V_{\text {calc }}$ is $2.6 \mathrm{ppm}$ larger than $V_{N}$. 


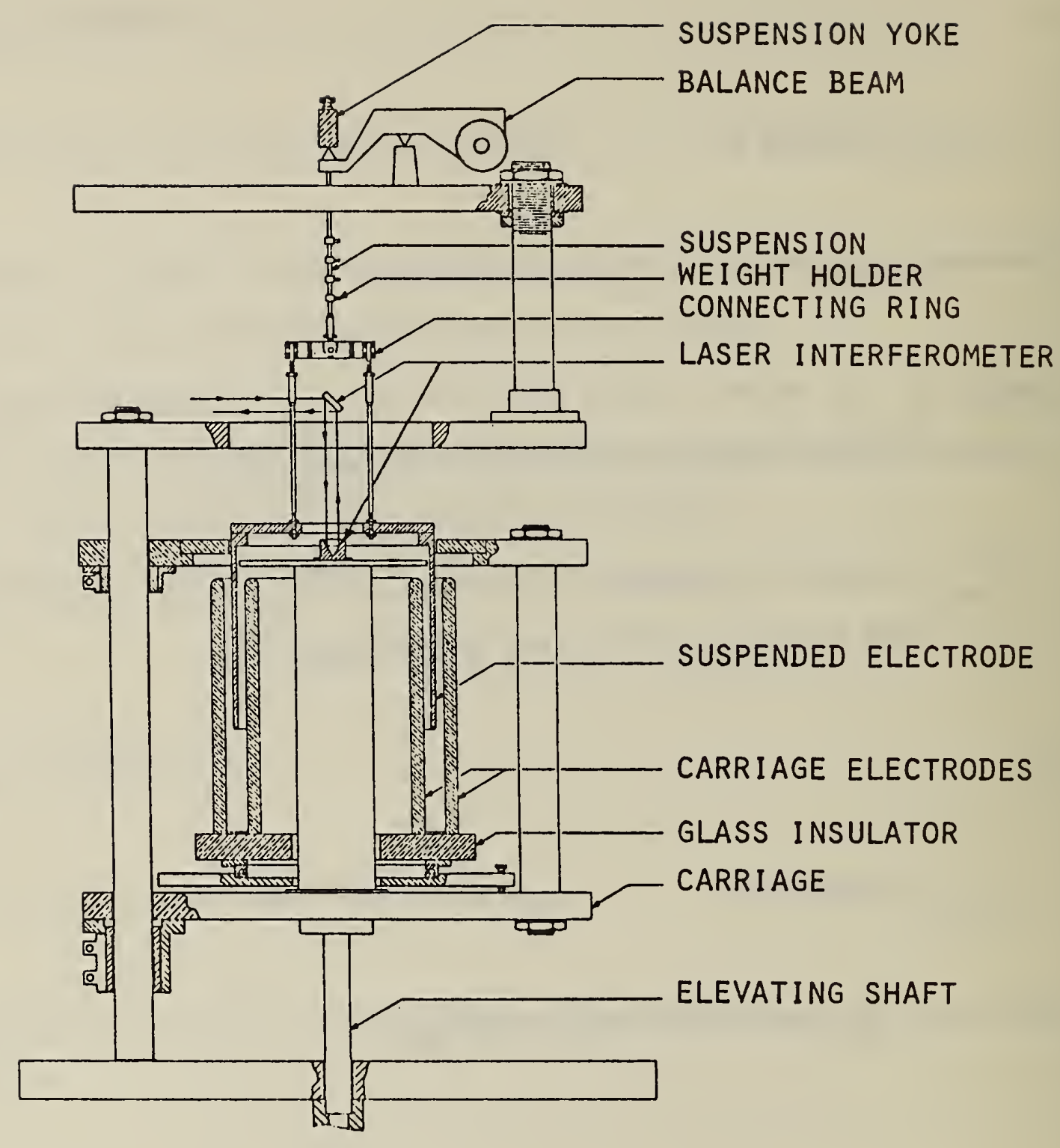

Fig. 1 Relevant features of the arrangement for the SI volt experinent. 


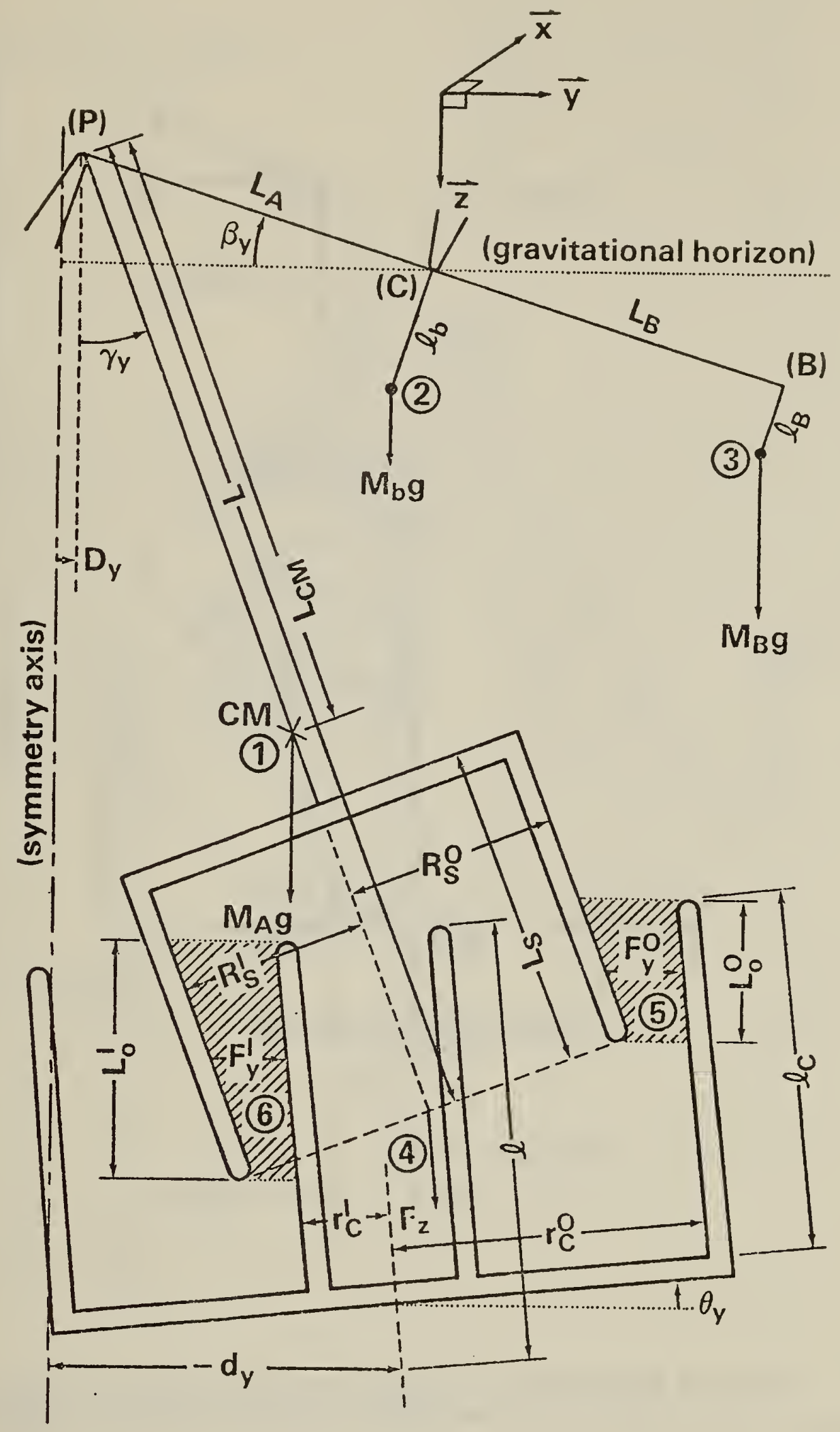

Fig. 2 Coordinate system and quantities required for the forces, the capacitances, and the equations of motion. 

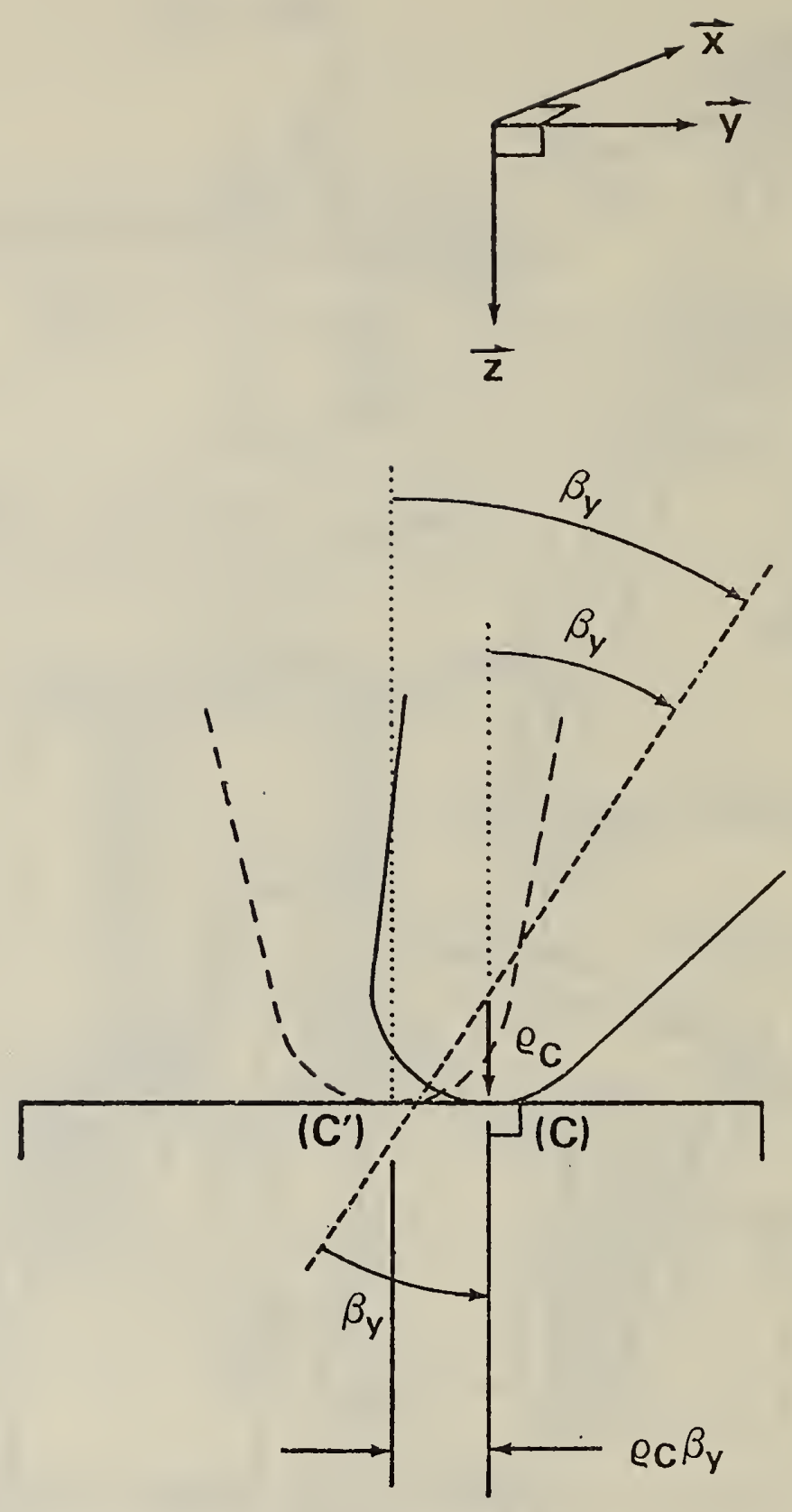

Fig. 3 Effect on balance beam of center knife rolling on its flat. 


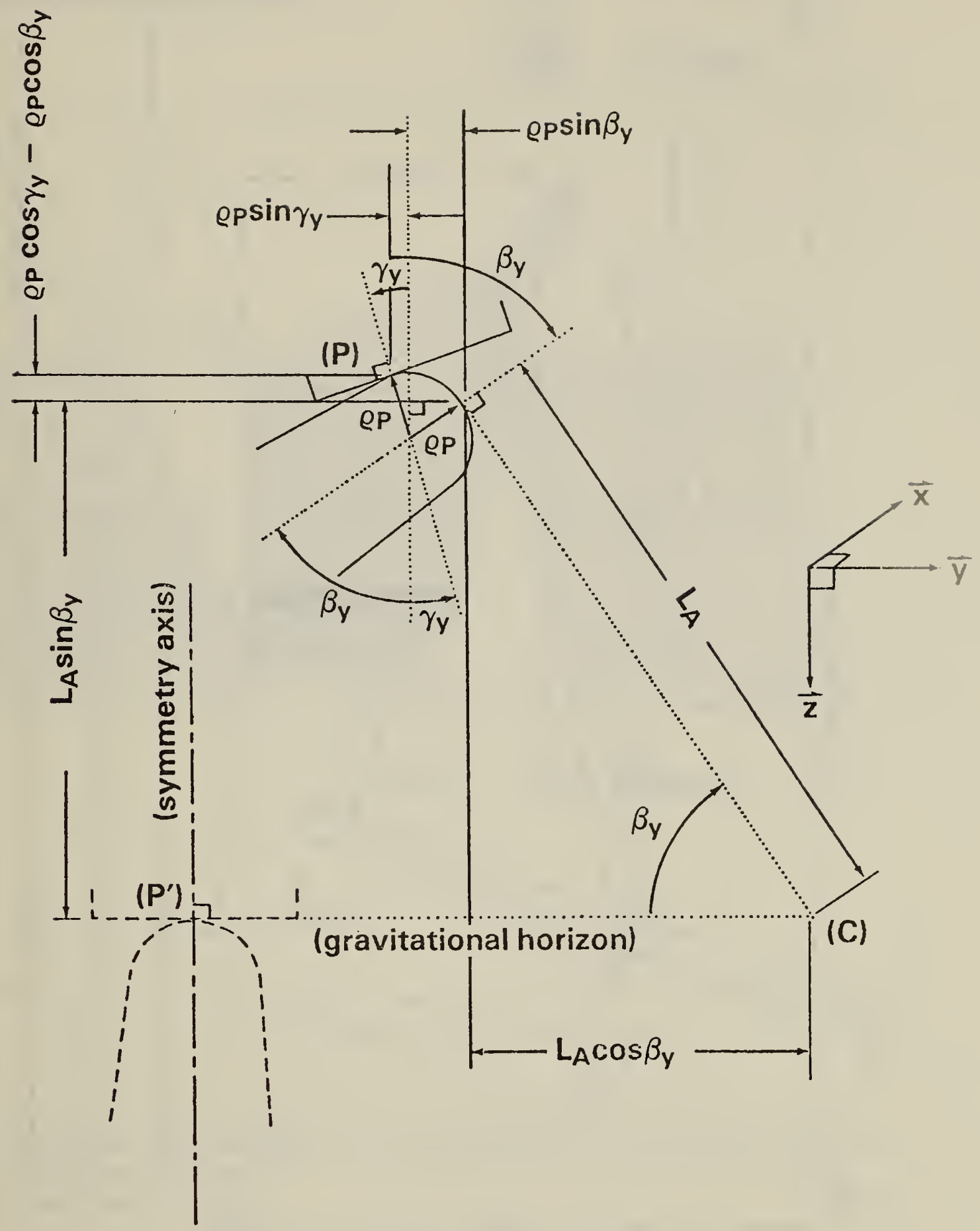

Fig. 4 Effect on balance beam of pendulum flat rolling on the load knife edge. 


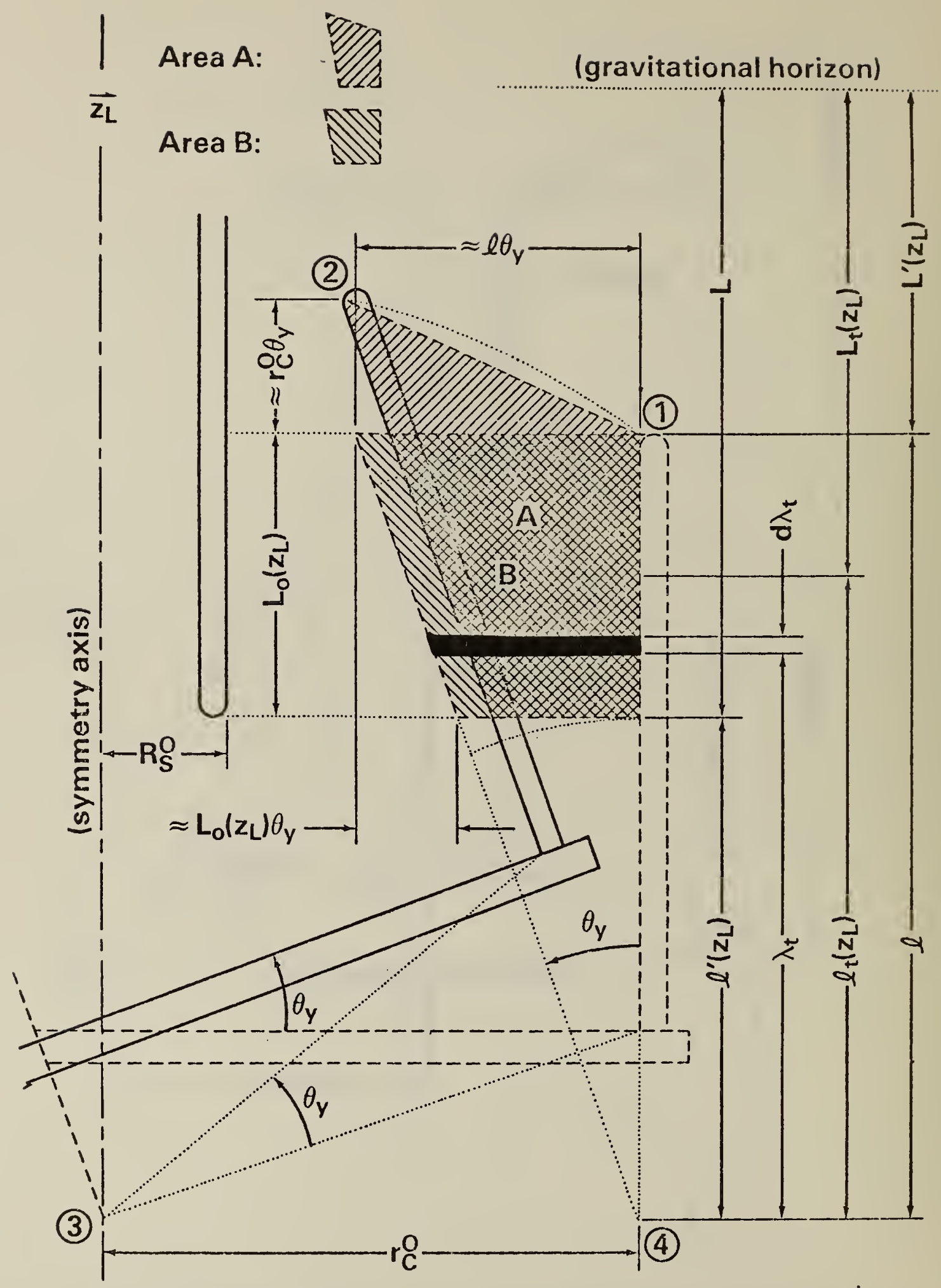

Fig. 5 Effect on capacitance of outer carriage electrode pivoting about point 3. 


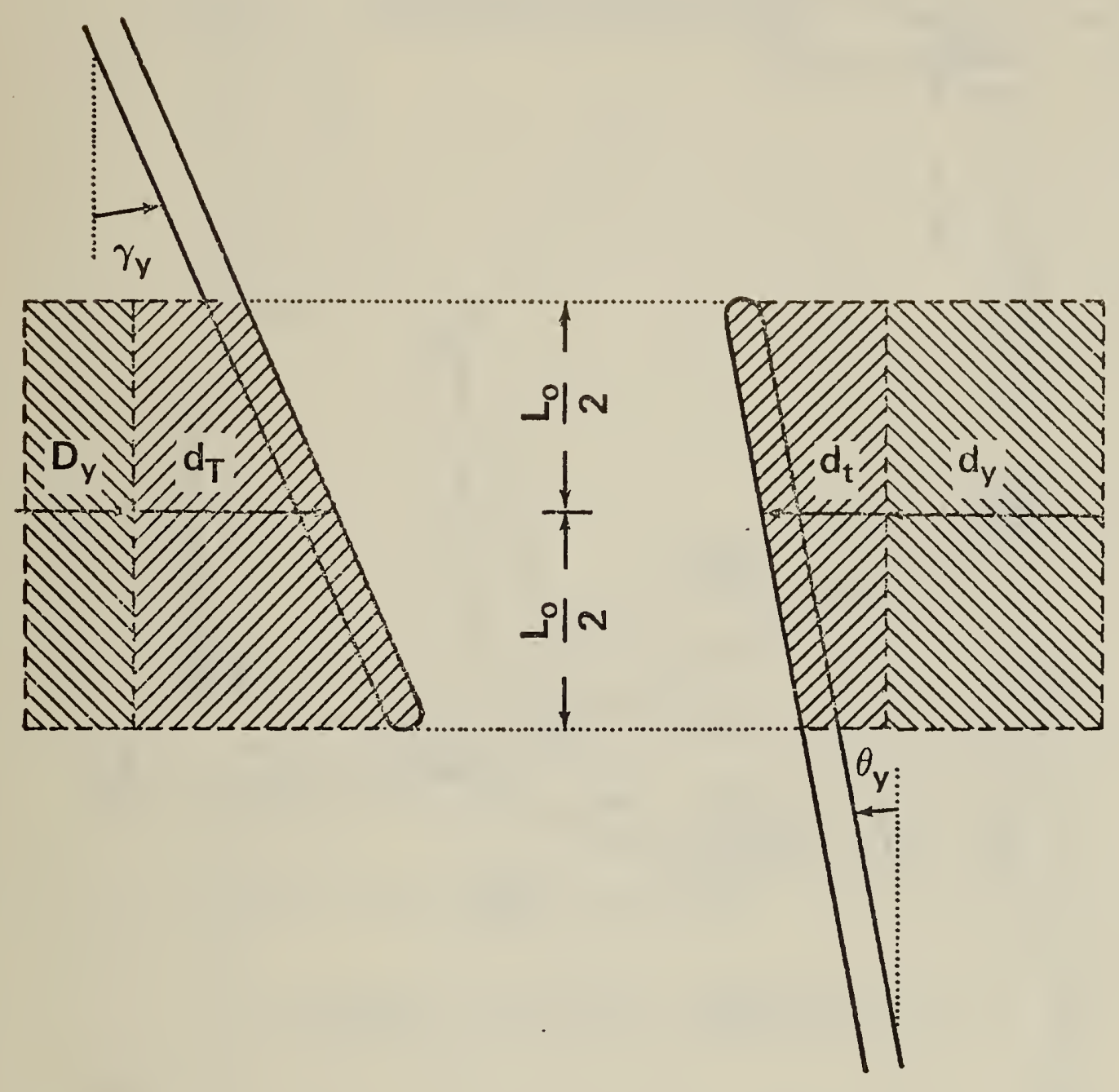

Fig. 6 Relative displacements of the suspended electrode and the outer carriage electrode. 


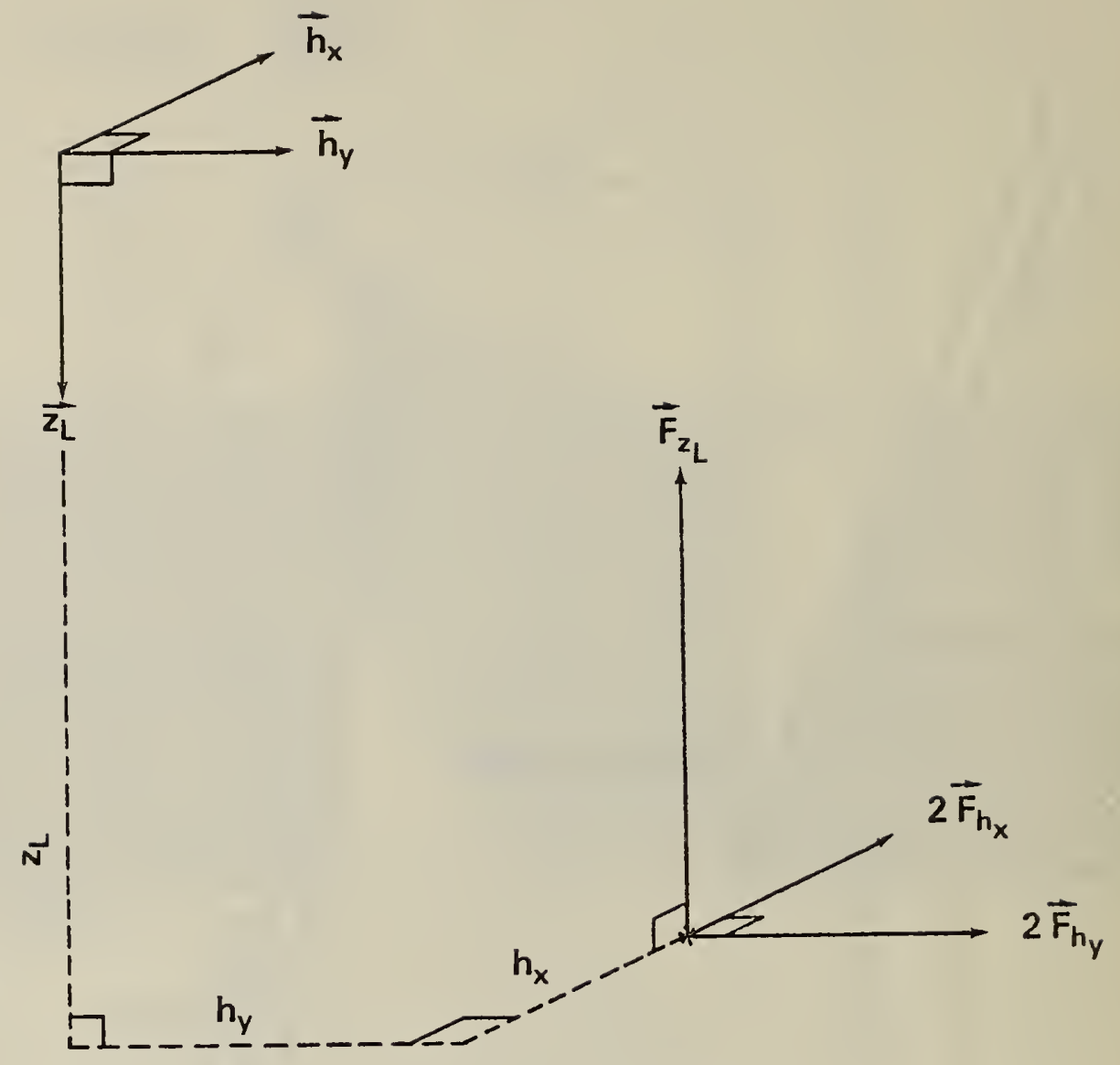

Fig. 7 Coordinate system for the relative electrode positions and the resulting electrical forces. 


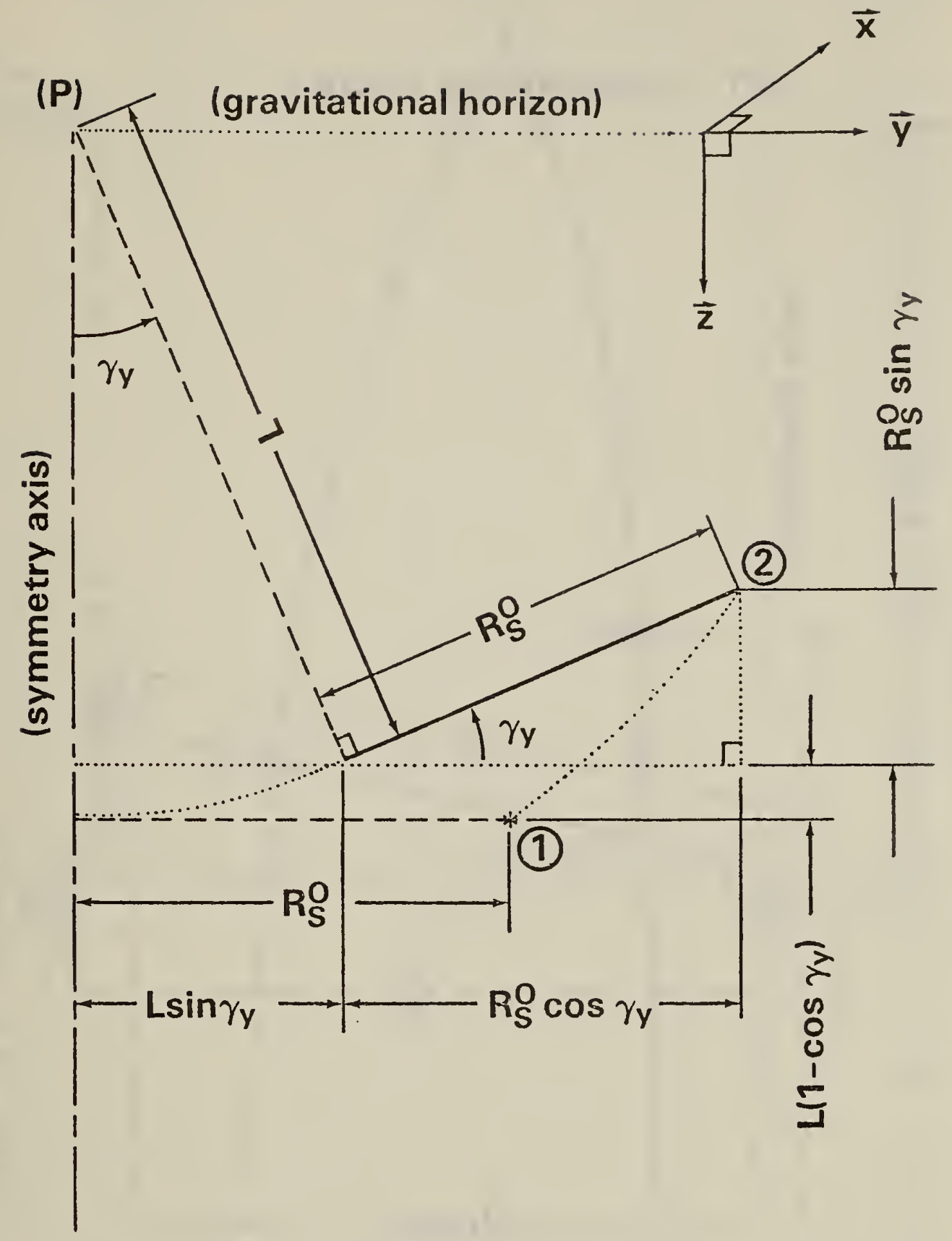

Fig. 8 Location of a point on the outer rim of the suspended electrode. 


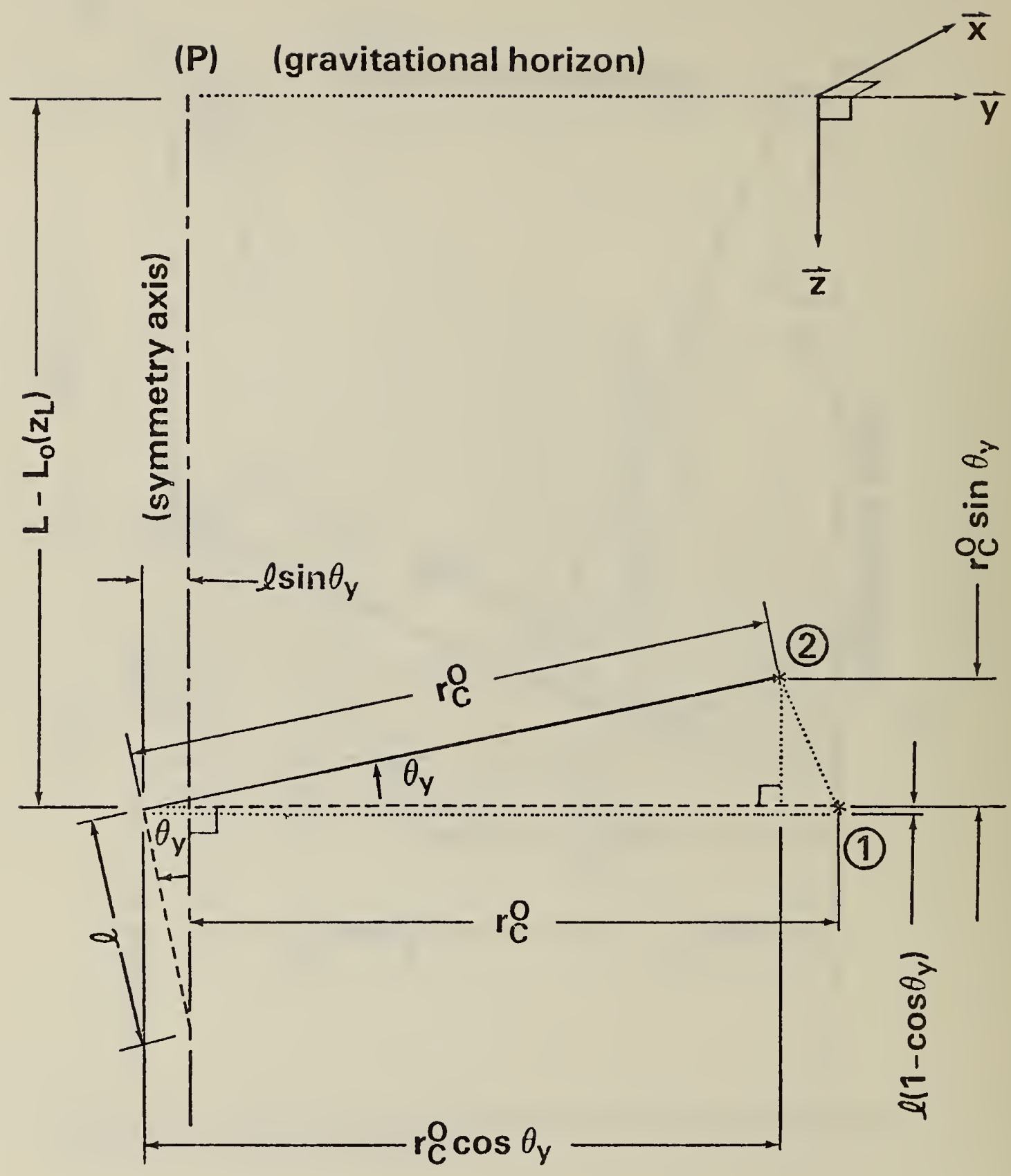

Fig. 9 Location of a point on the rim of the outer carriage electrode. 


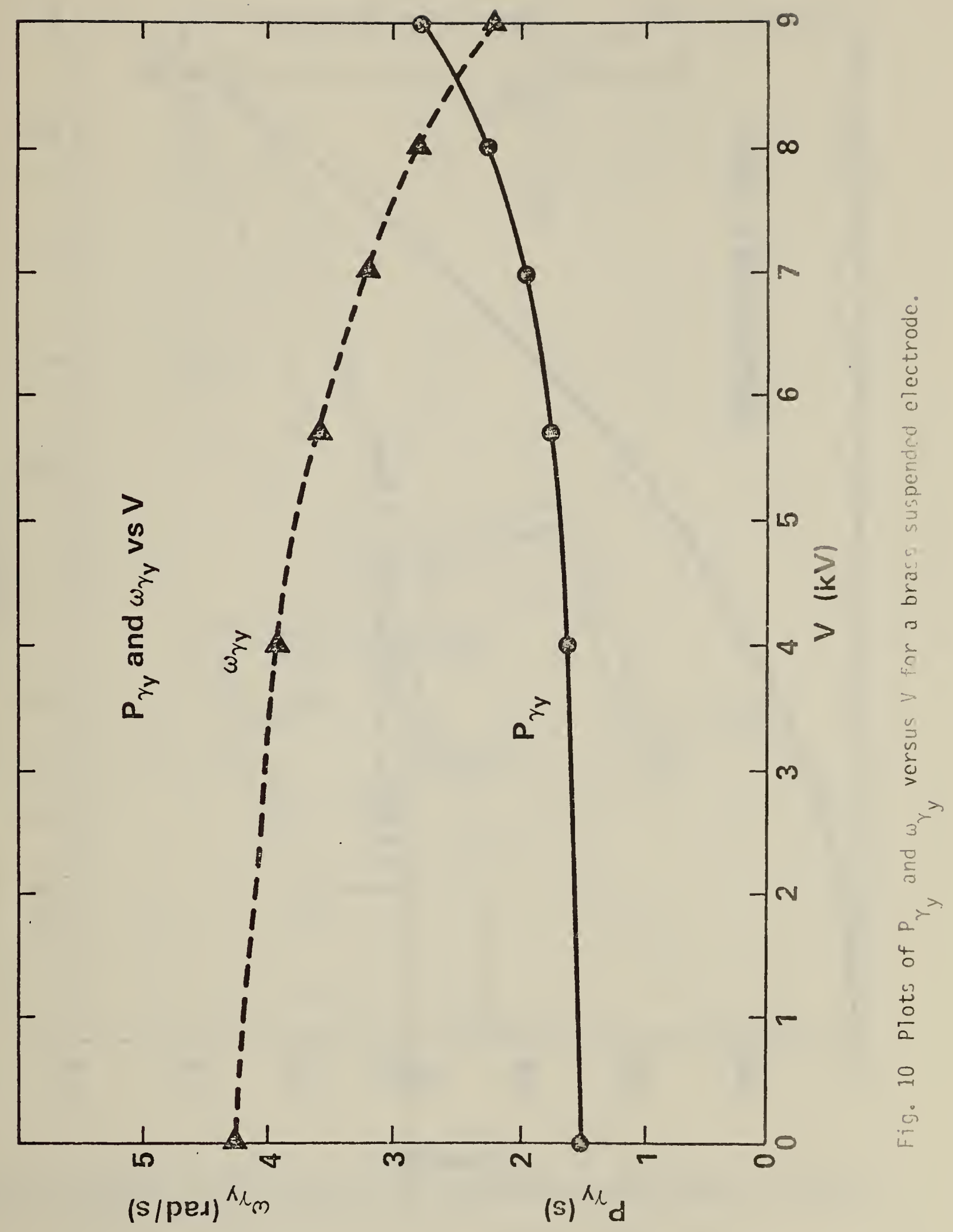




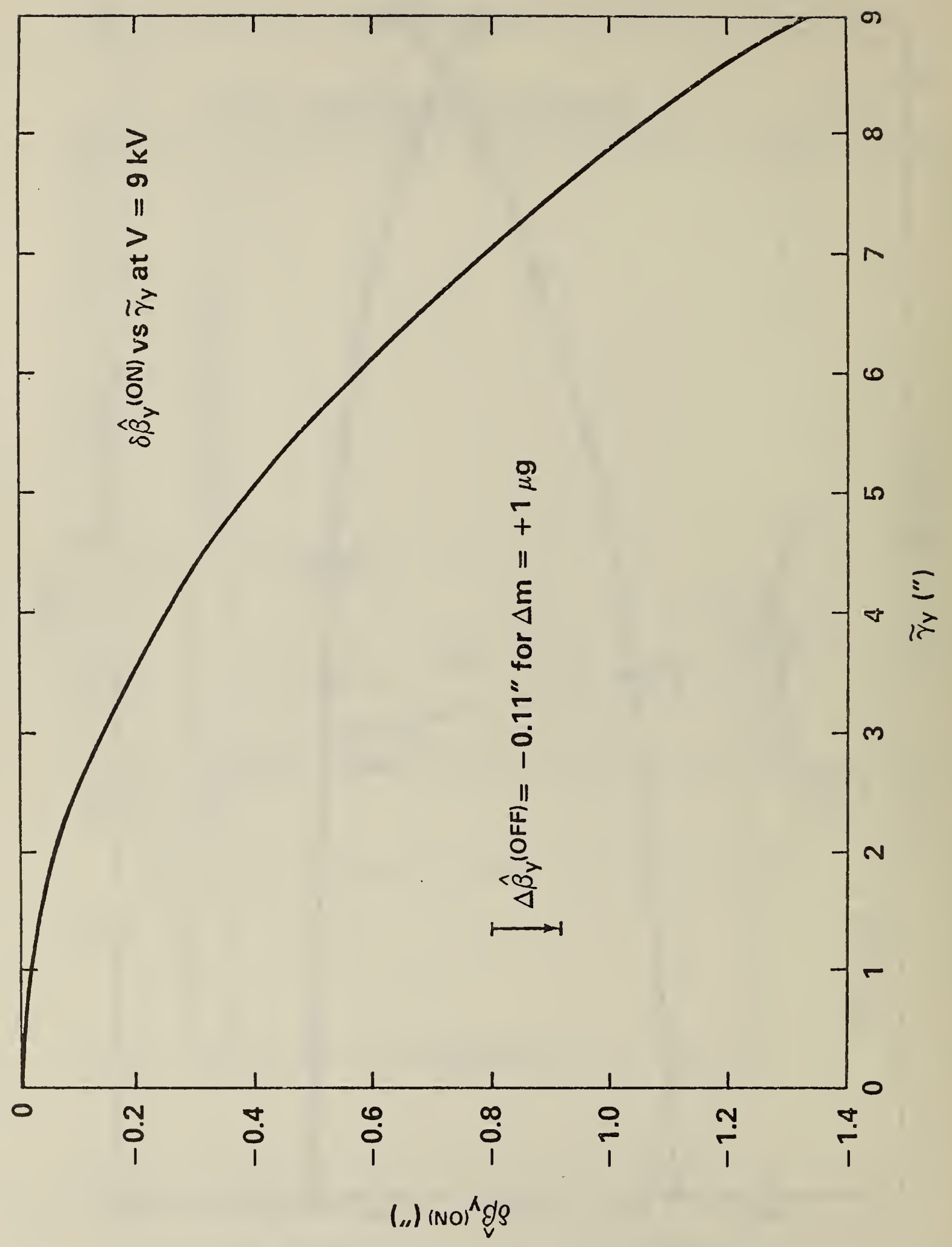




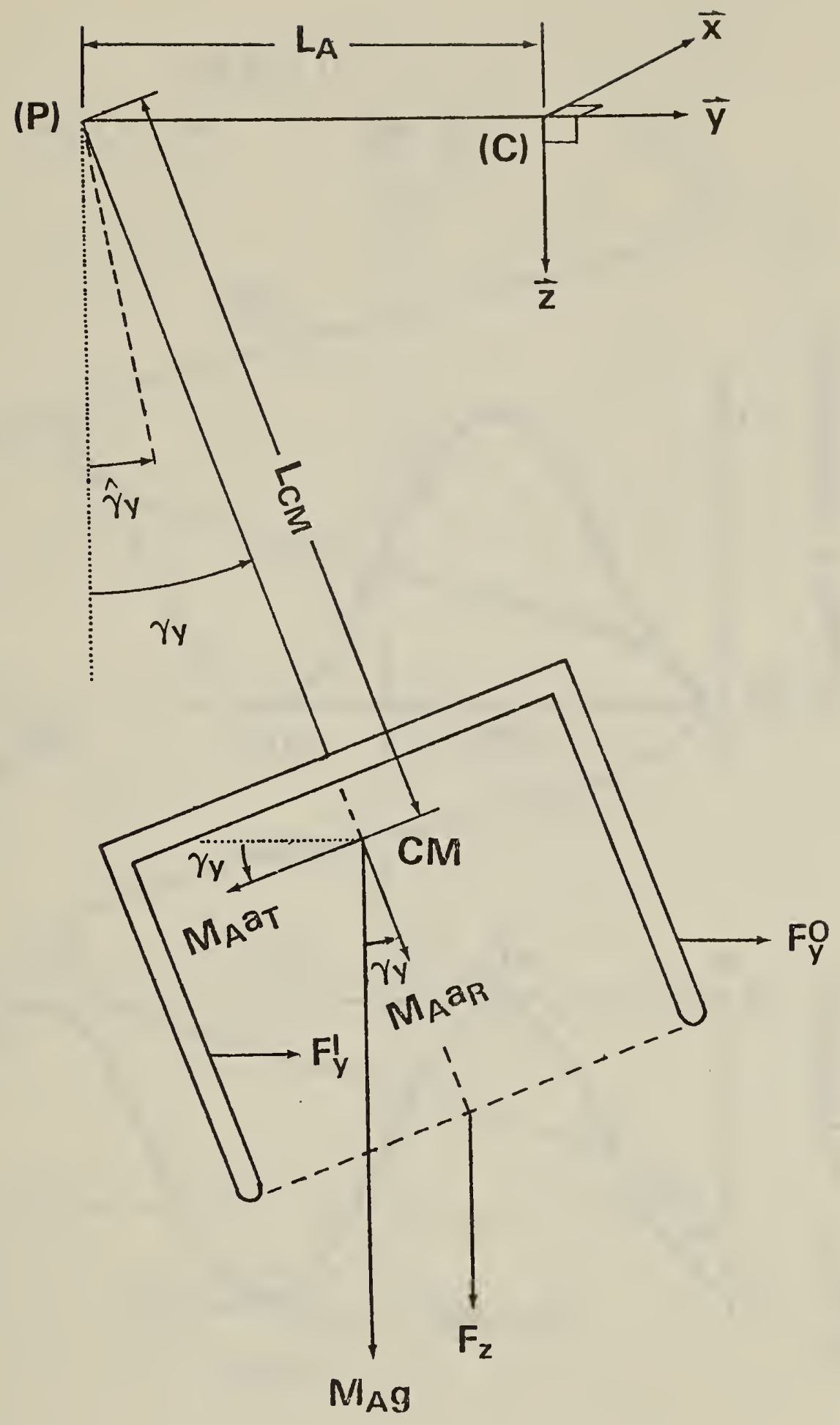

Fig. 12 External forces induced on the suspended electrode when the balance beam is at rest. 


$$
\begin{gathered}
\mathrm{V}=0 \mathrm{kV} \\
\hat{\gamma}_{\mathrm{y}}=0 \quad \mathrm{P}_{\gamma_{\mathrm{y}}}=1.5 \mathrm{~s}
\end{gathered}
$$

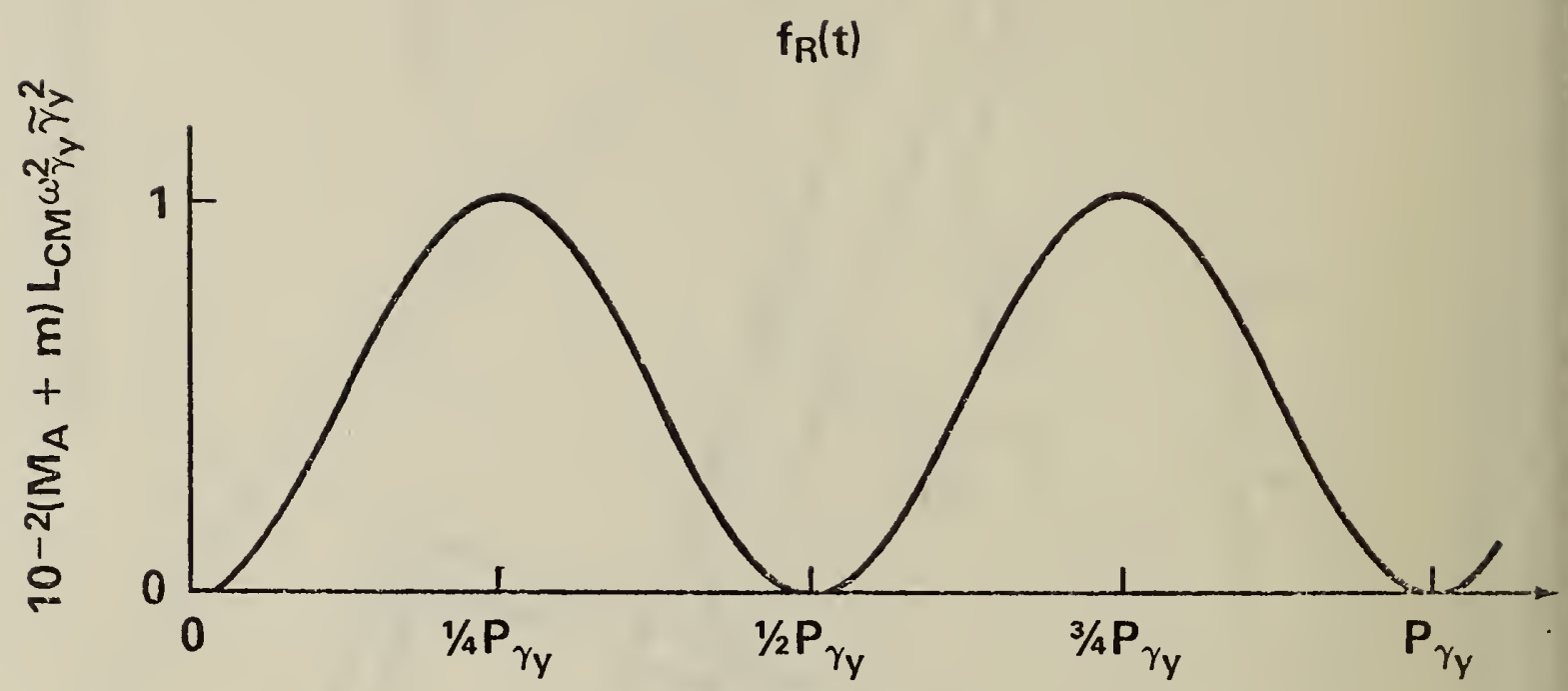

$t(s)$

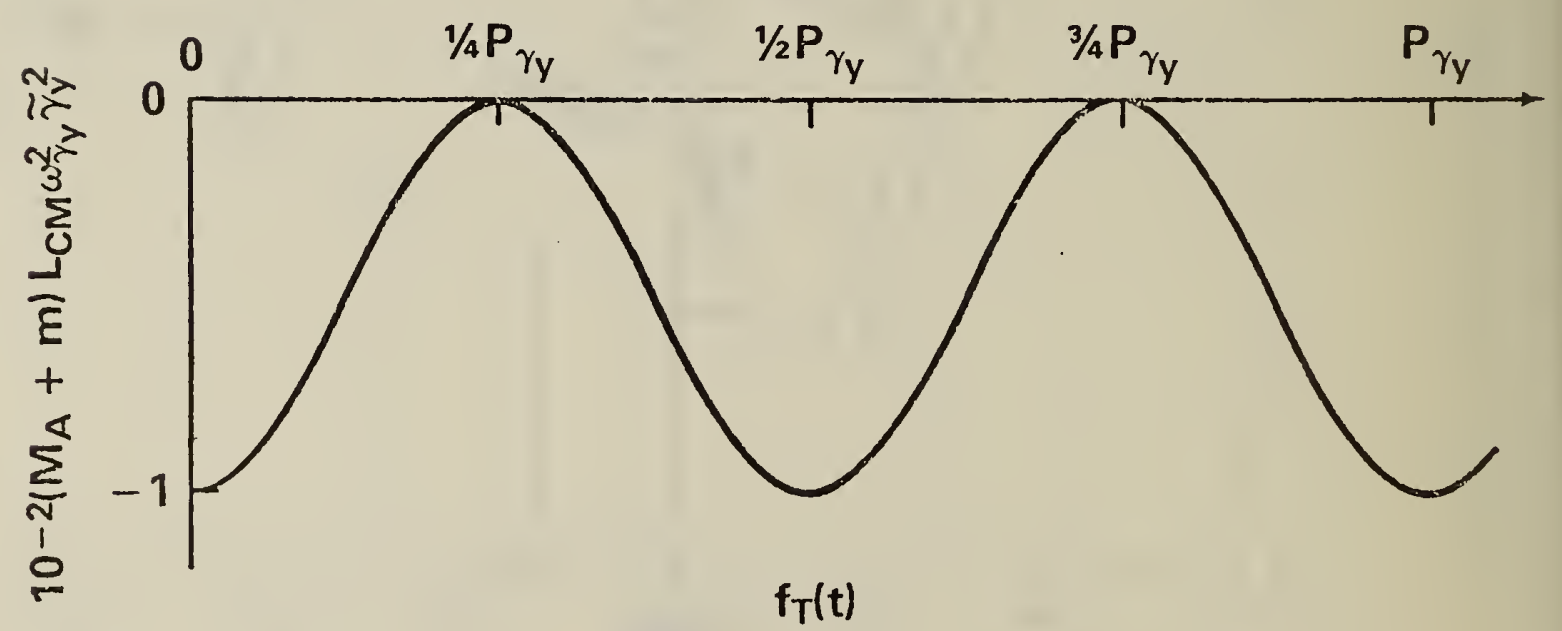

Fig. 13 Plots of $f_{R}$ and $f_{T}$ versus time, with voltage off, for one pendulum period, which is $1.5 \mathrm{~s}$ for the brass electrode. $f_{R}=+5.08 \times 10^{-8} \mathrm{~N}$ at $t=(1 / 4) P_{\gamma_{y}}$ if $\tilde{\gamma}_{y}=3.30 \times 10^{-5} \mathrm{rad}\left(6.8^{\prime \prime}\right)$. 


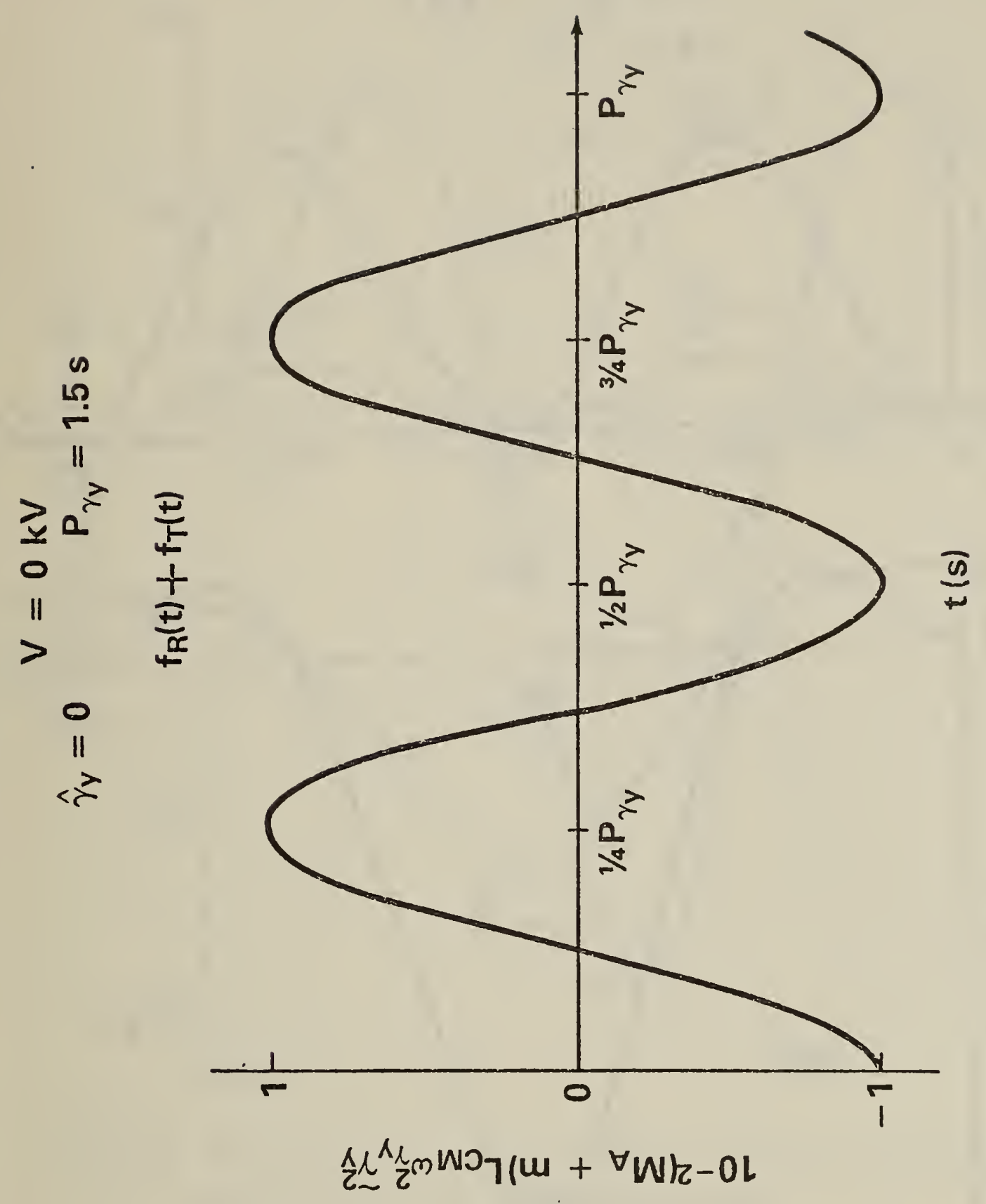

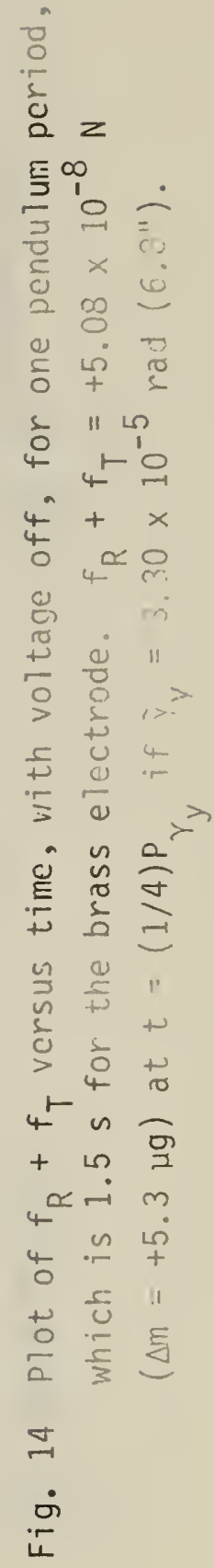




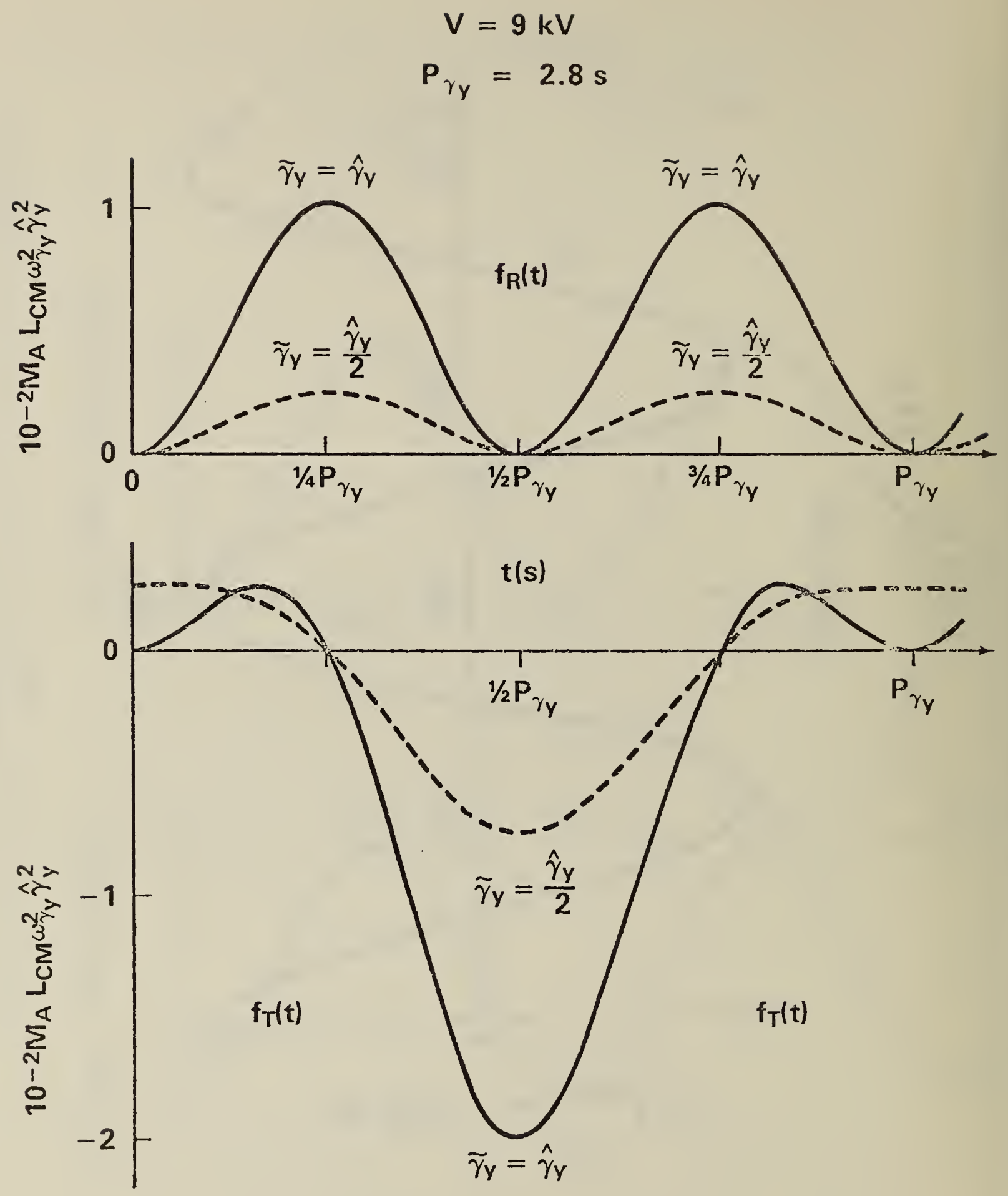

Fig. 15 Plots of $f_{R}$ and $f_{T}$ versus time at $V=9 k V$ for one pendulum period, which is $2.8 \mathrm{~s}$ for the brass electrode. $f_{R}=+1.38 \times 10^{-8} \mathrm{~N}$ at $t=$ $(1 / 4) P_{\gamma_{y}}$ if $\tilde{\gamma}_{y}=\hat{\gamma}_{y}=3.30 \times 10^{-5} \mathrm{rad}\left(6.8^{\prime \prime}\right)$. 


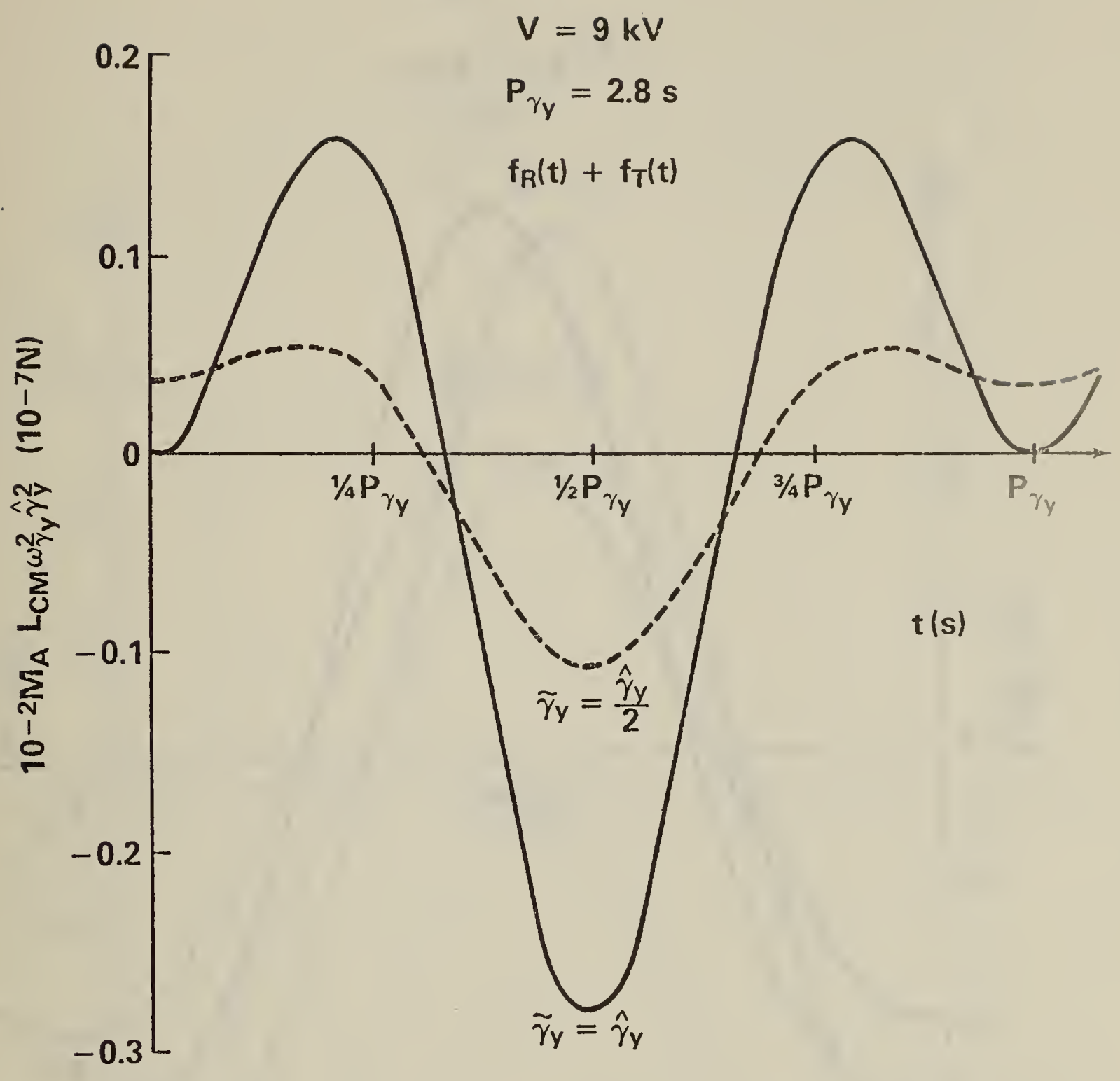

Fig. 16 Plot of $f_{R}+f_{T}$ versus time at $V=9 k V$ for one pendulum period, which is $2.8 \mathrm{~s}$ for the brass electrode. 


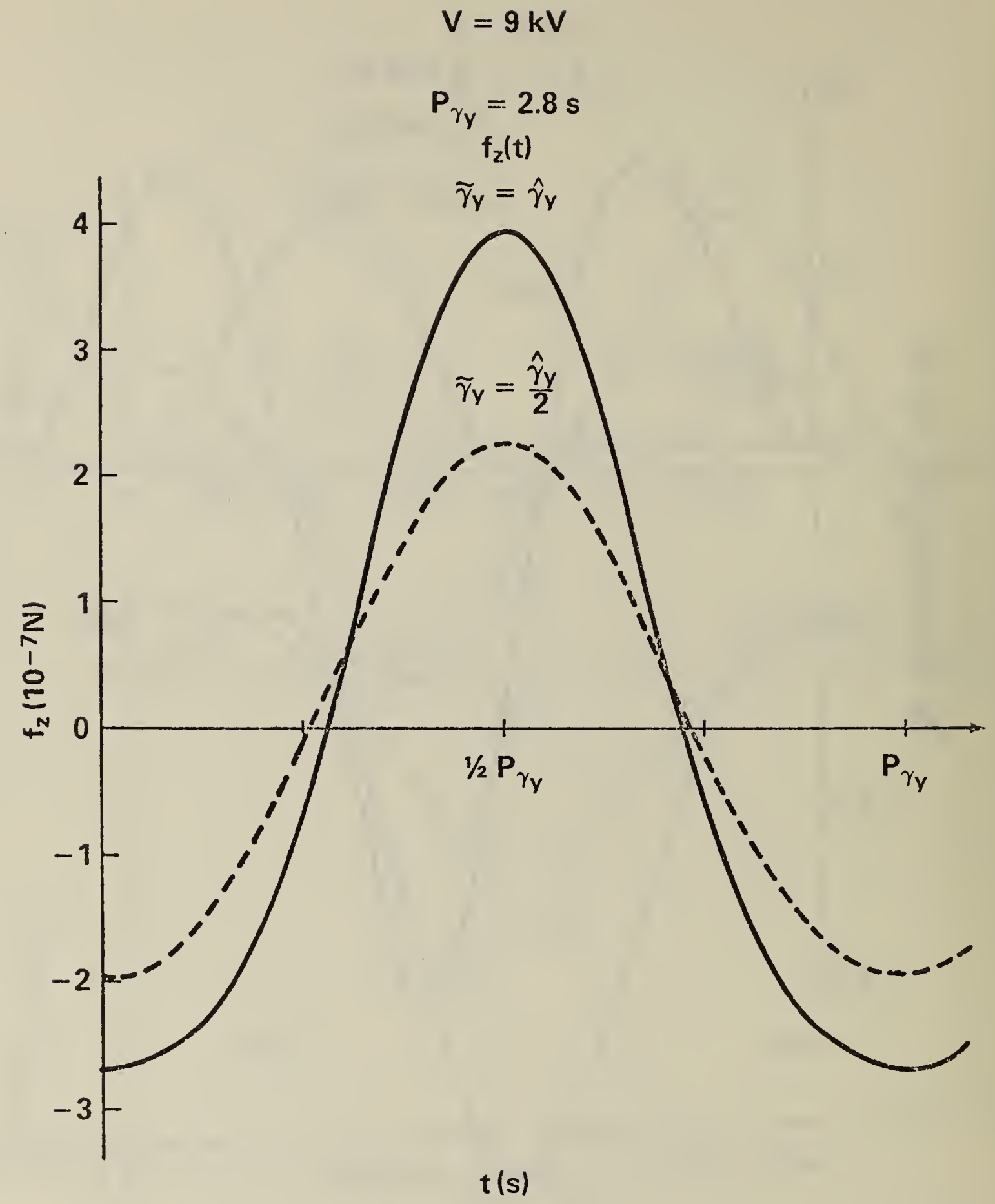

Fig. 17 Plot of $f_{z}$ versus time at $V=9 \mathrm{kV}$ for one pendulum period, which is $2.8 \mathrm{~s}$ for the brass electrode. 


$$
\begin{gathered}
V=9 \mathrm{kV} \\
P_{\gamma_{Y}}=2.8 \mathrm{~s}
\end{gathered}
$$

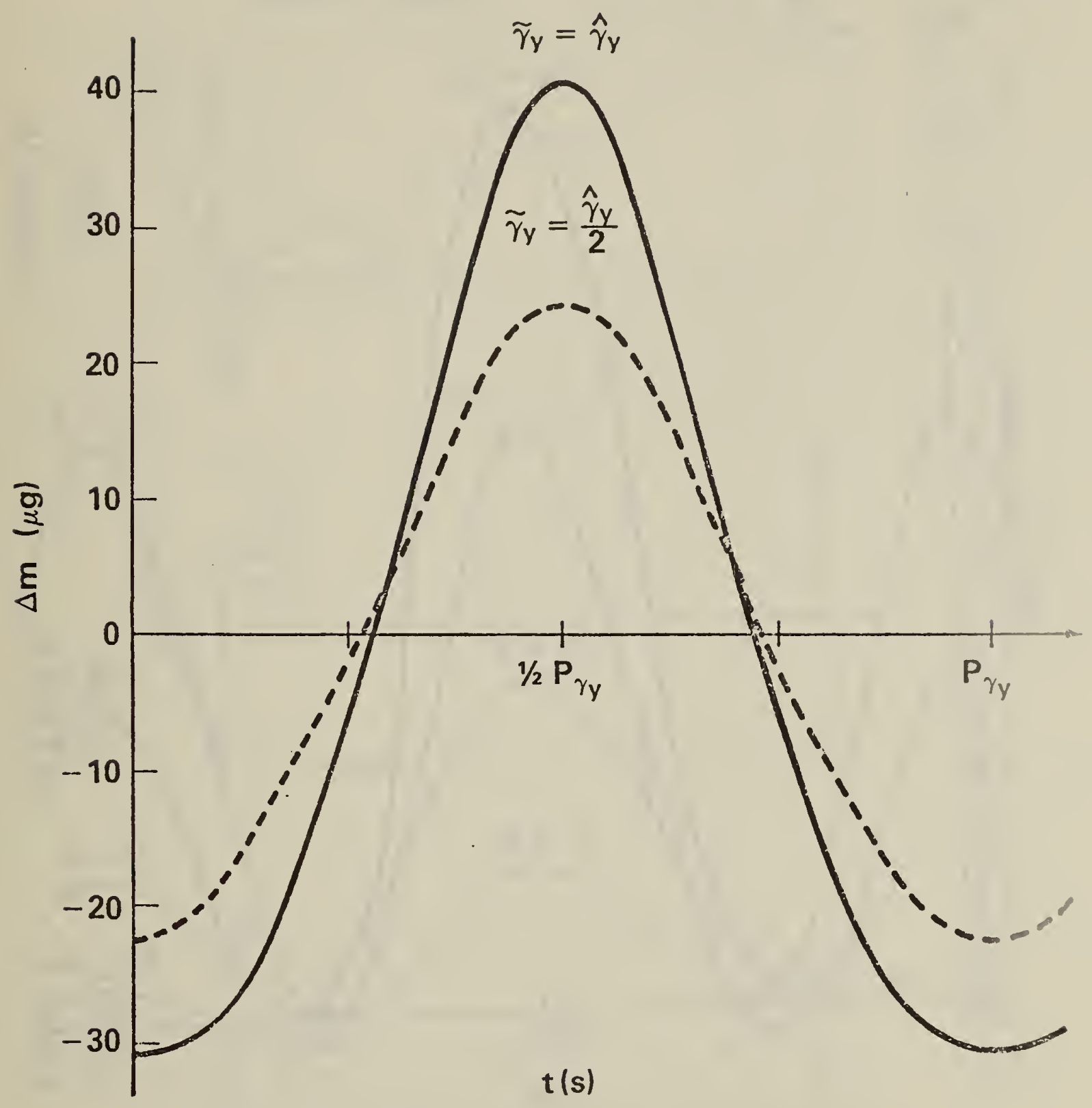

Fig. 18 Plot of $\Delta m$ versus time at $V=9 \mathrm{kV}$ for one pendulum period, which is $2.8 \mathrm{~s}$ for a brass electrode. $\Delta \mathrm{m}$ is defined by Equation $\mathrm{R}-10$. 


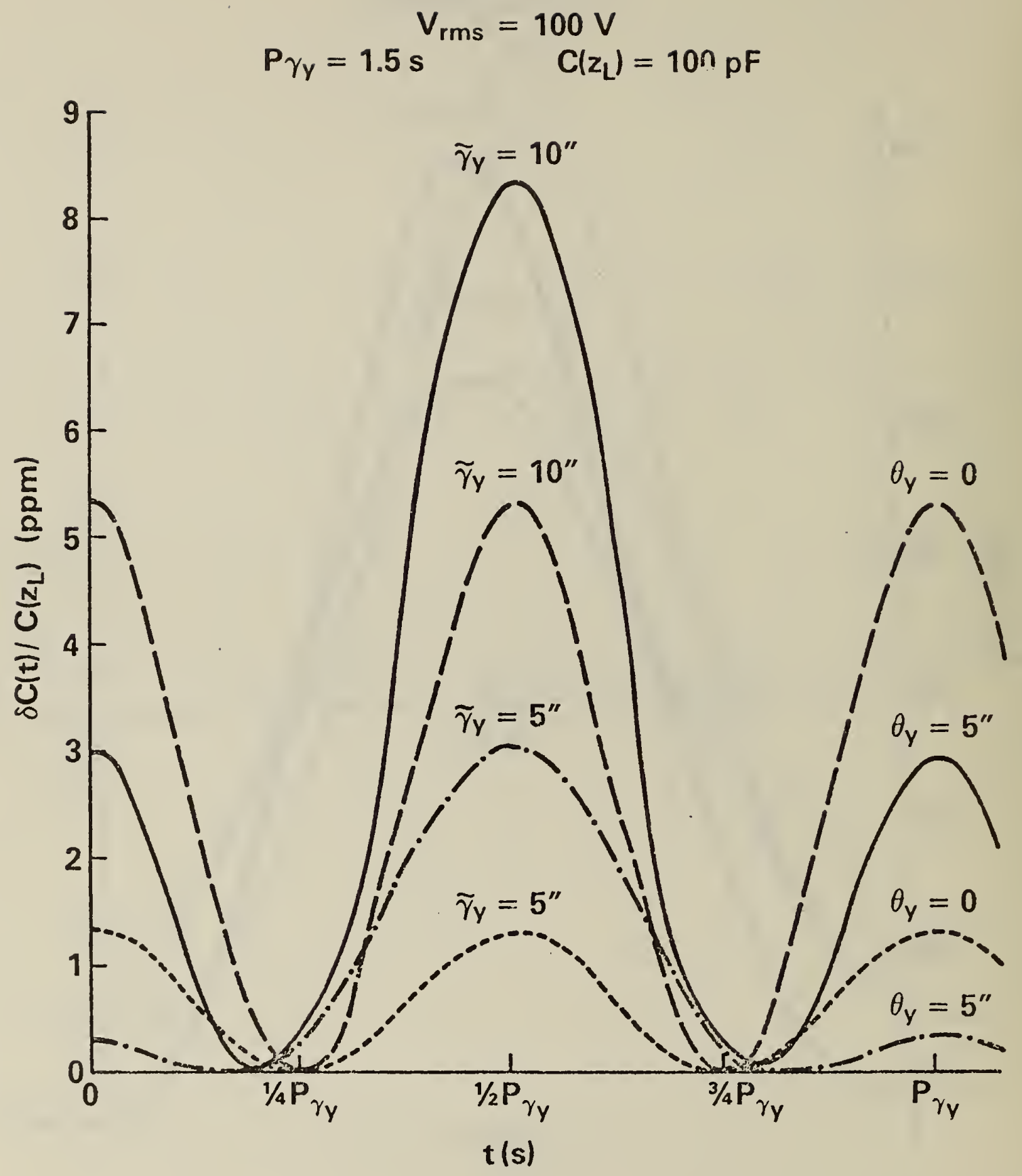

Fig. 19 Plot of $\delta C(t) / C\left(z_{L}\right)$ versus time at $V_{r m s}=100 \mathrm{~V}$ and $C\left(z_{L}\right)=100 \mathrm{pF}$ for one pendulum period, which is $1.5 \mathrm{~s}$ for a brass electrode. $\delta C(t) / C\left(z_{L}\right)$ is defined by Equations $S-3$ and $S-4$. 

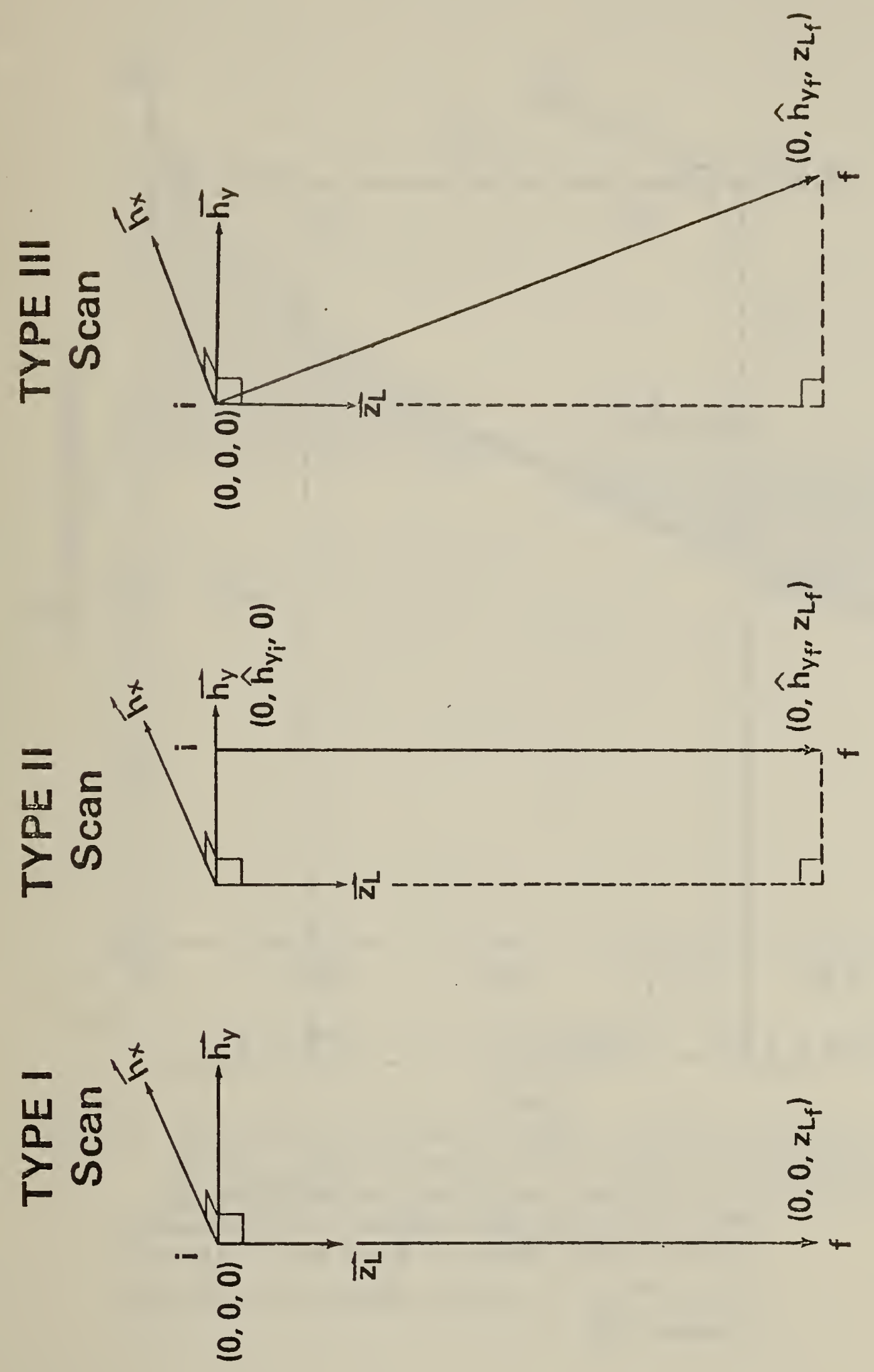

40

4.2

ठ $\frac{0}{2}$

ए

ट

오

i 


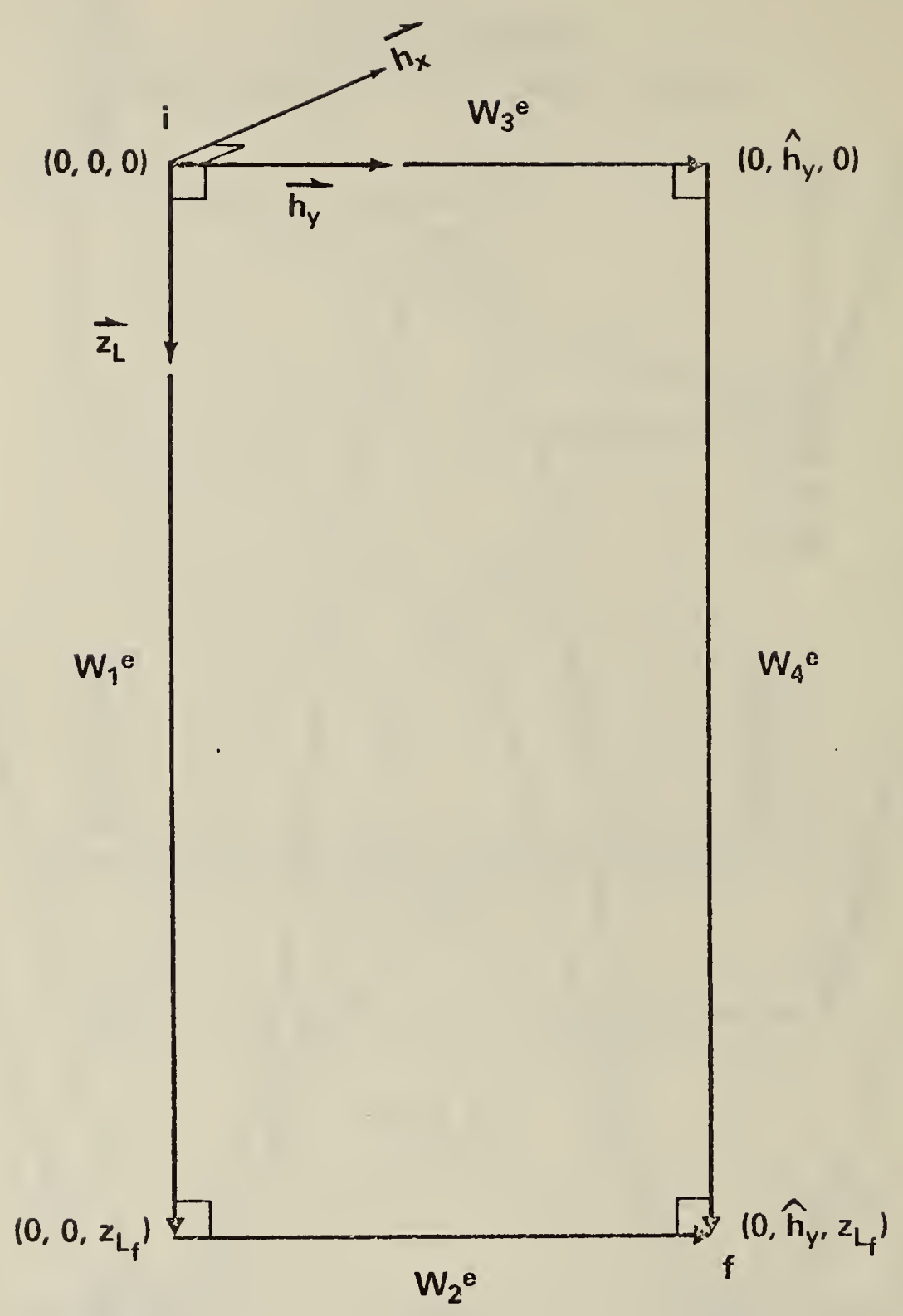

Fig. 21 Two different paths used in Appendix $W$ to verify that the path integral is conservative. These are the values of $\hat{h}_{y}$ at high voltages, so $d_{y}$ and/or $\theta_{y}$ are not constant for the path integral $\mathrm{w}_{4}^{\mathrm{e}}$. 


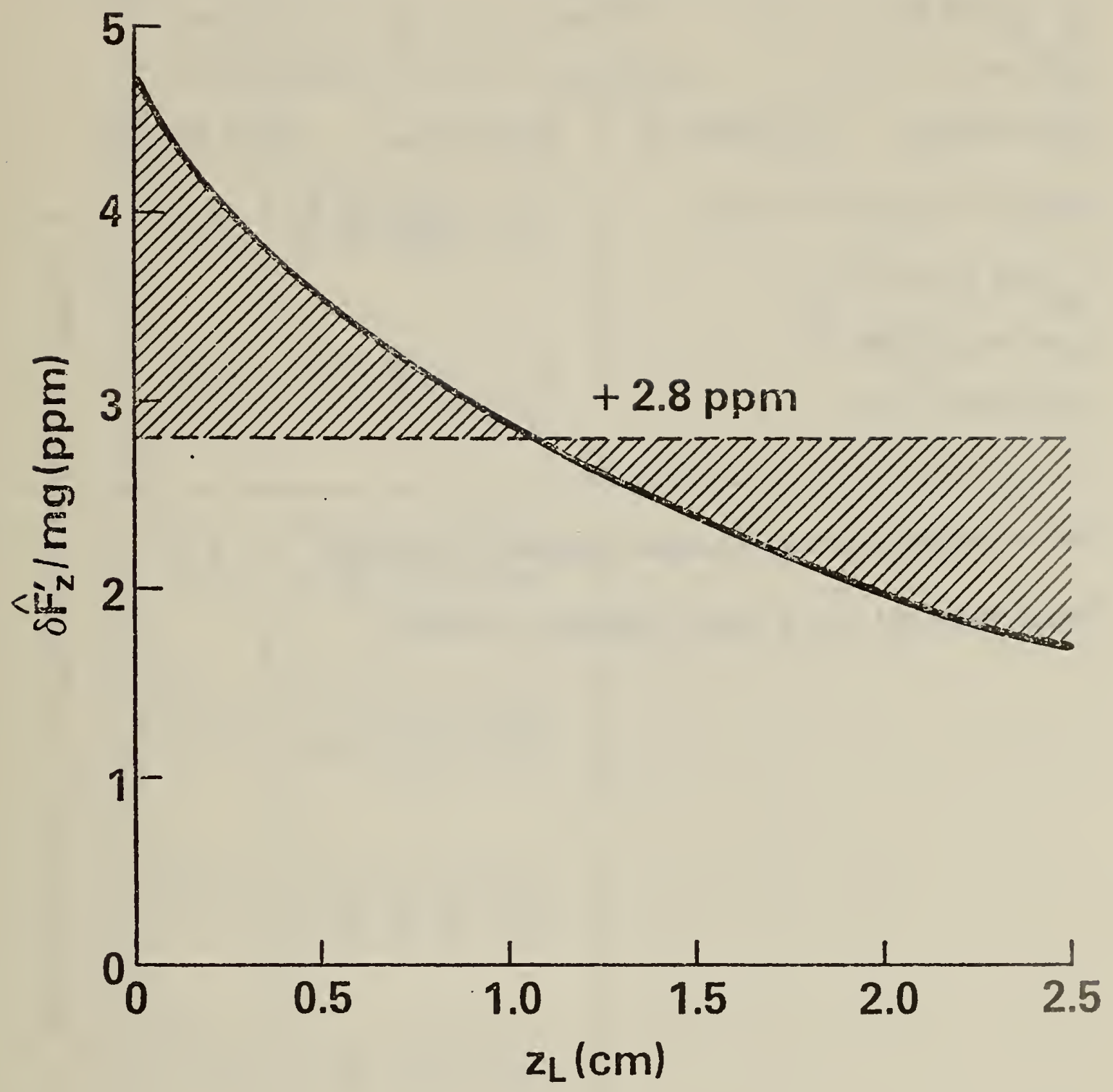

Fig. 22 Determination of the straight line which yiclds the same change in path integral as the curved line used in Example? of Appendix $Y$. 
Table 1. Length and mass values of quantities shown in Figures 2-4.

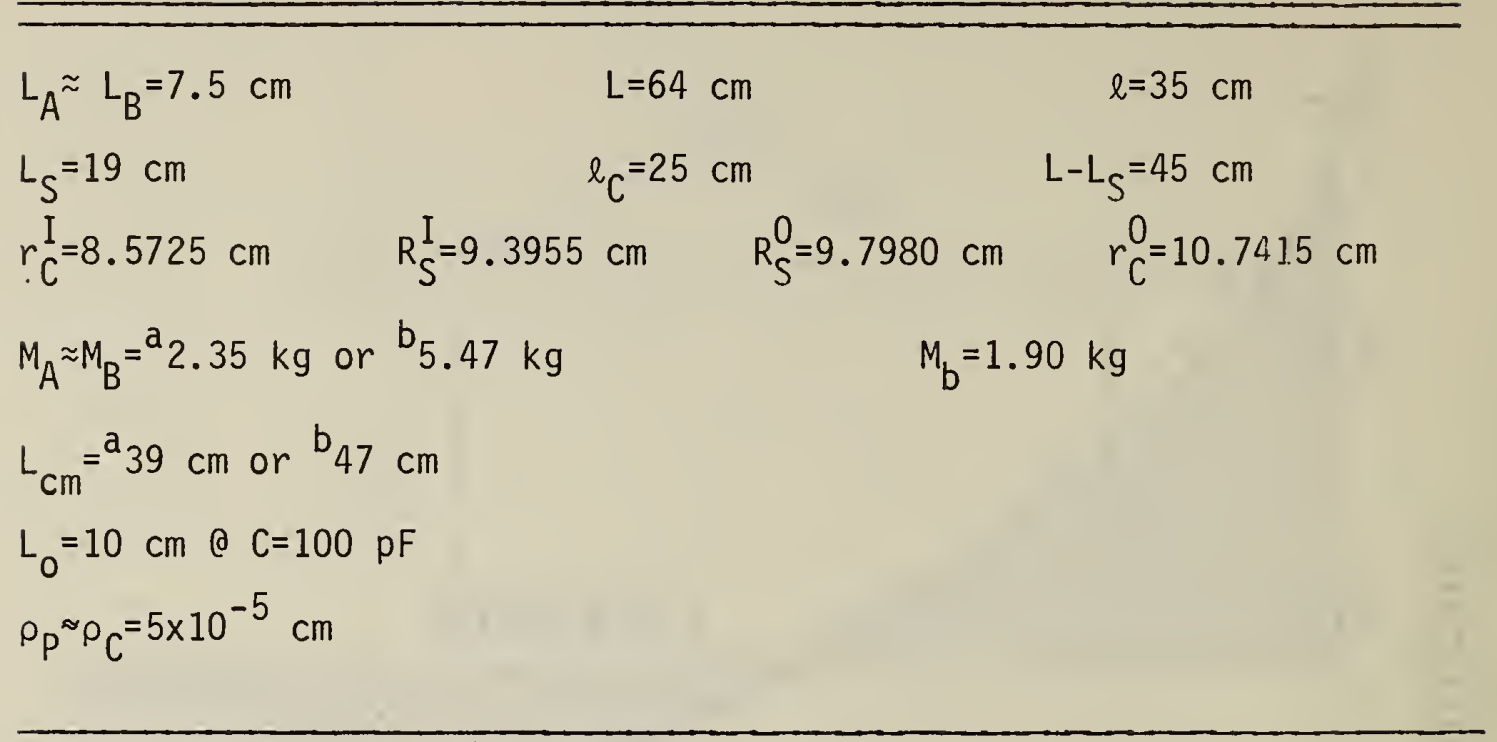

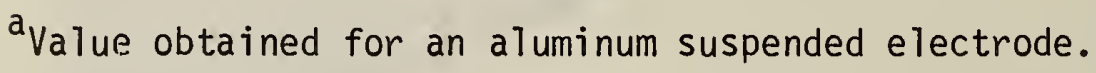

${ }^{b}$ Value obtained for a brass suspended electrode. 


\begin{tabular}{|c|c|}
\hline & 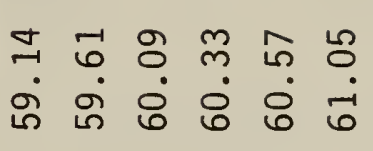 \\
\hline & 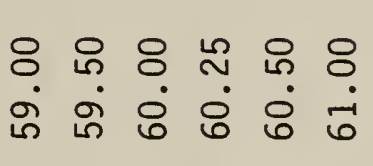 \\
\hline & 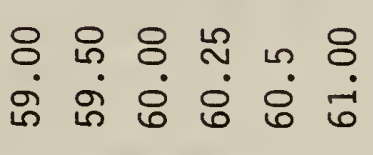 \\
\hline & 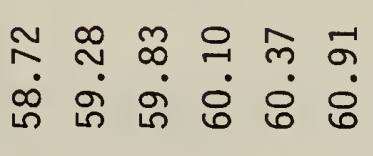 \\
\hline & 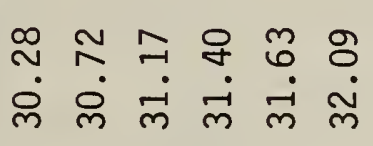 \\
\hline & 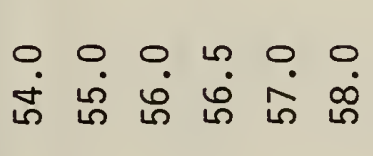 \\
\hline & $\stackrel{\sim}{\sim} \stackrel{\sim}{\sim} \stackrel{\infty}{\sim} \Omega$ \\
\hline & 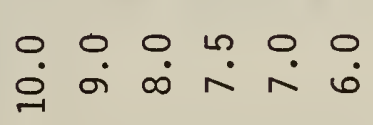 \\
\hline$\frac{N}{U}$ & 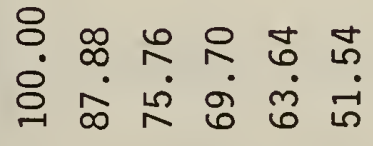 \\
\hline & $0 \rightarrow \sim \stackrel{n}{\sim} m \sigma$ \\
\hline
\end{tabular}




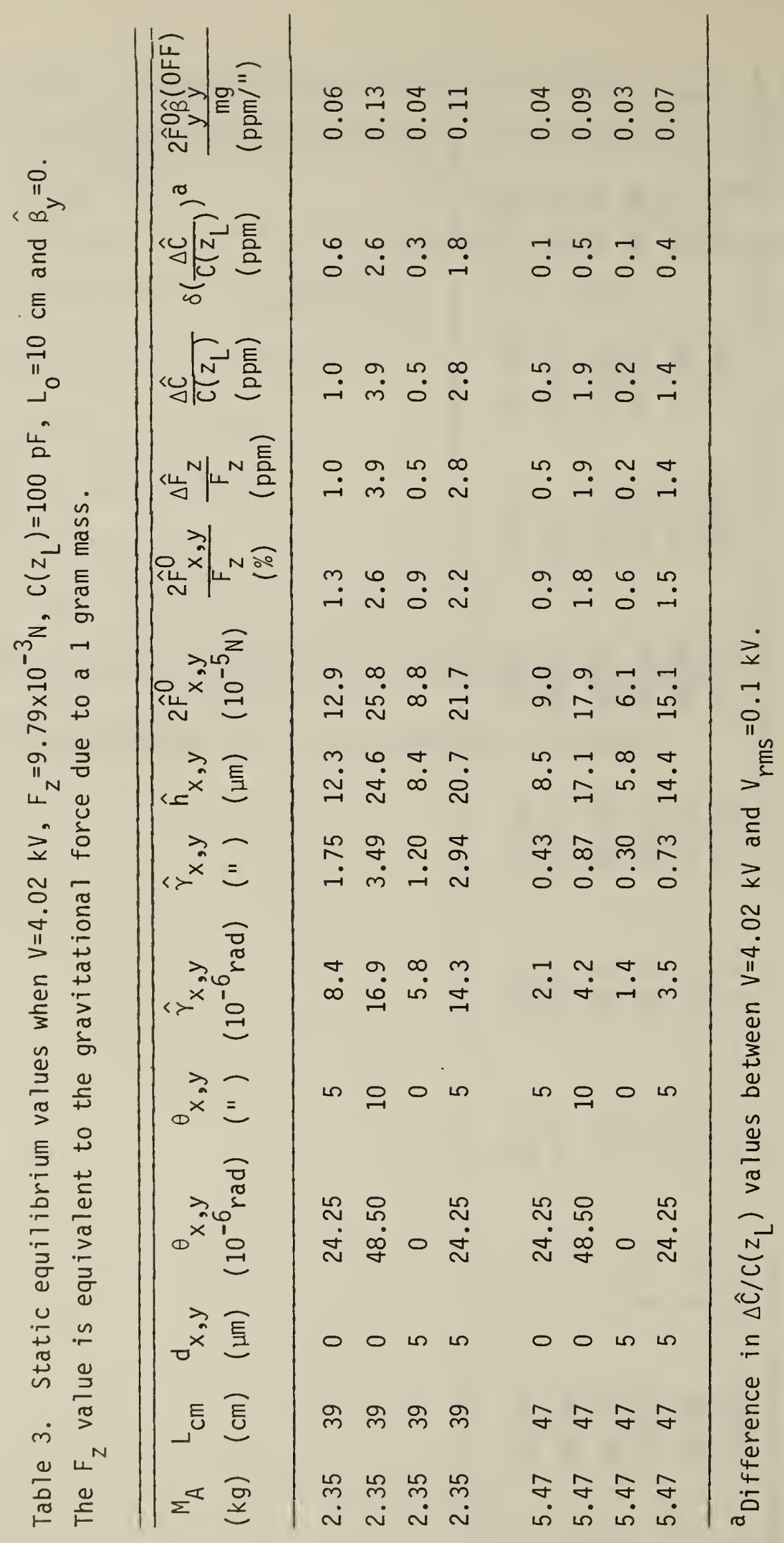




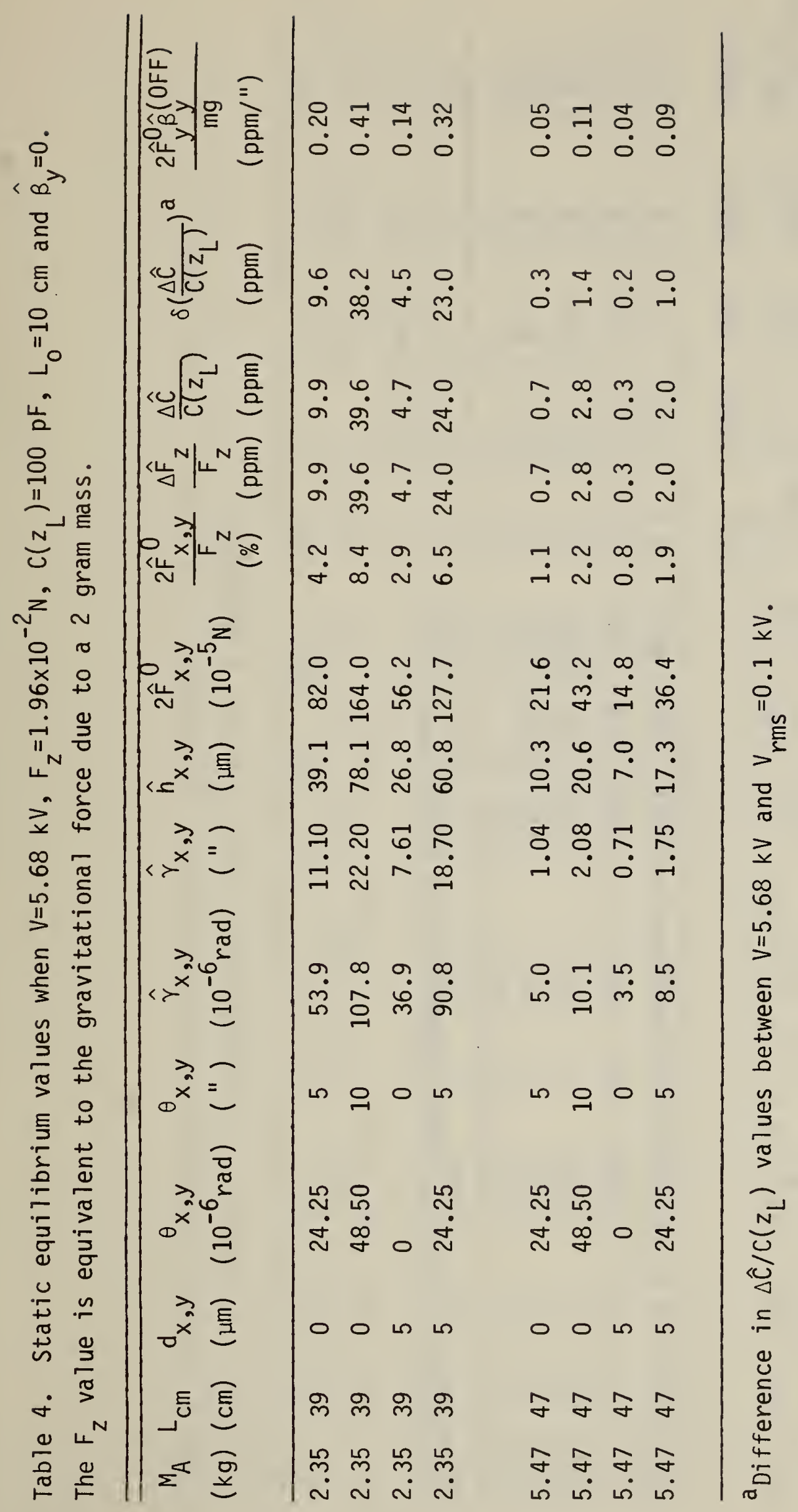




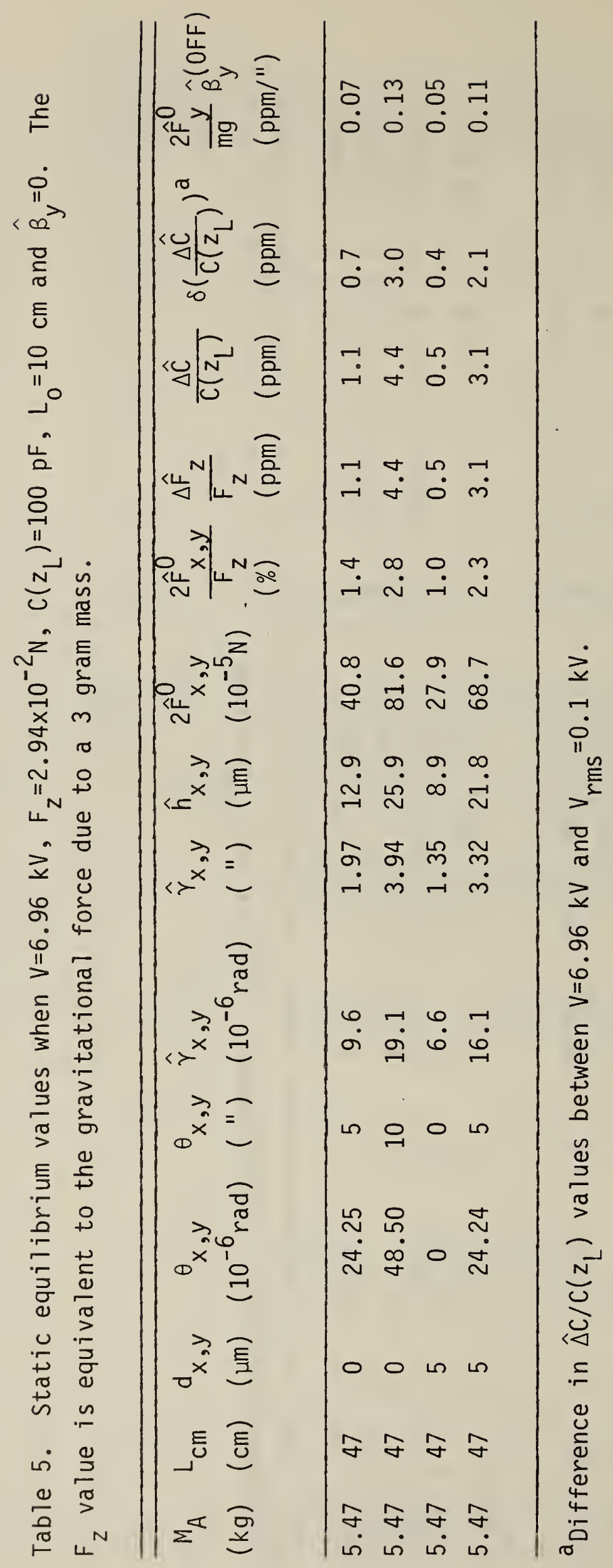




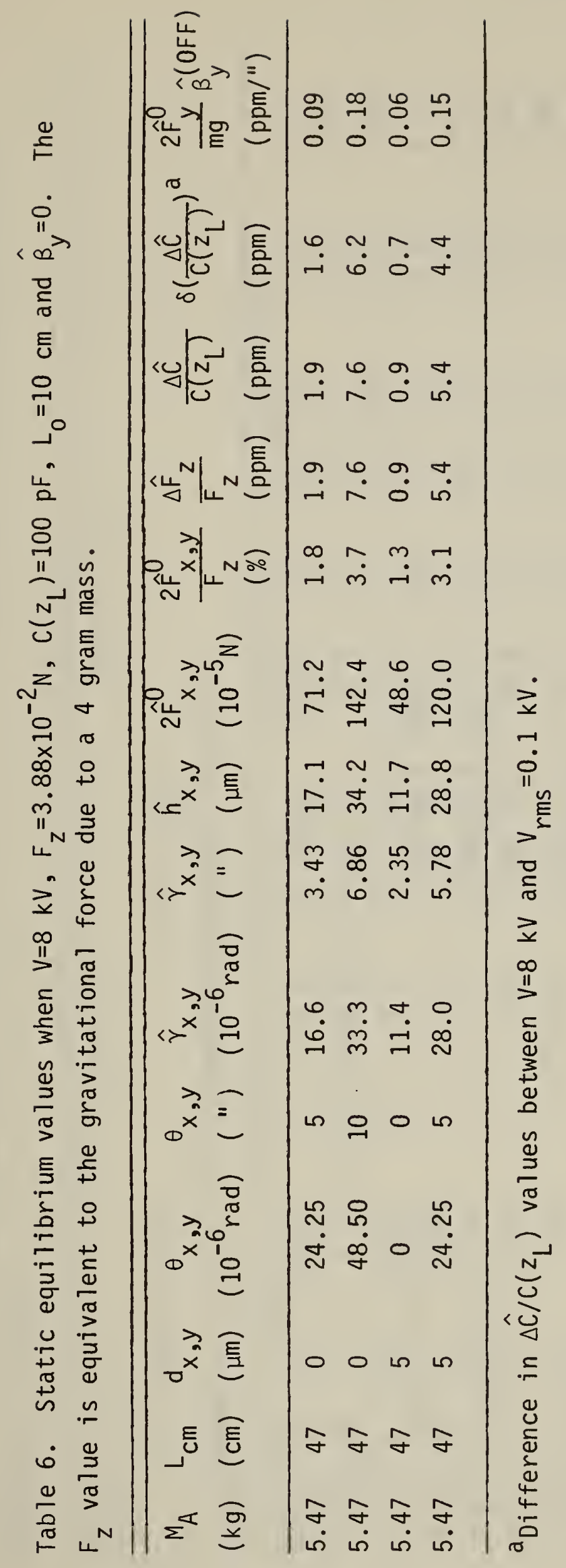




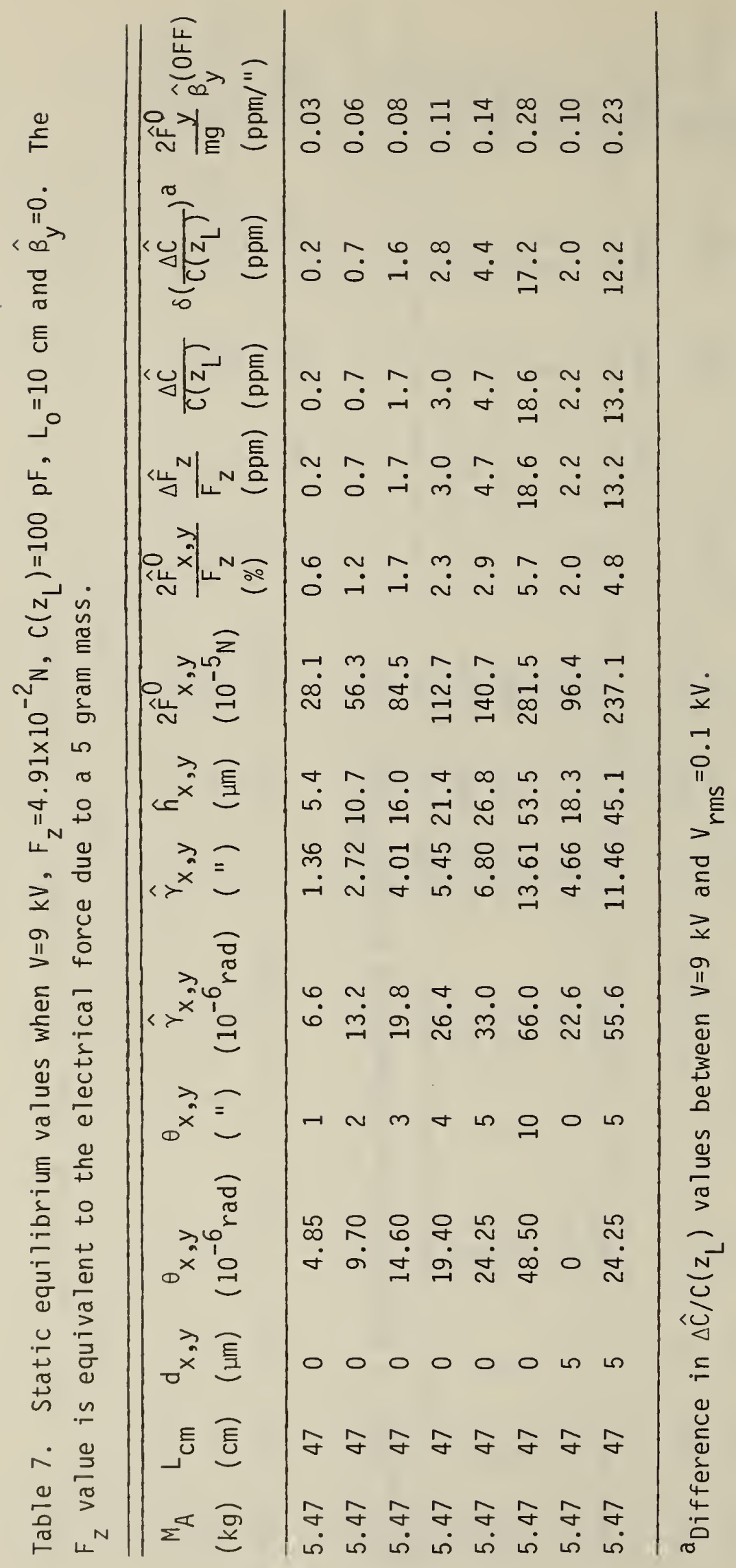




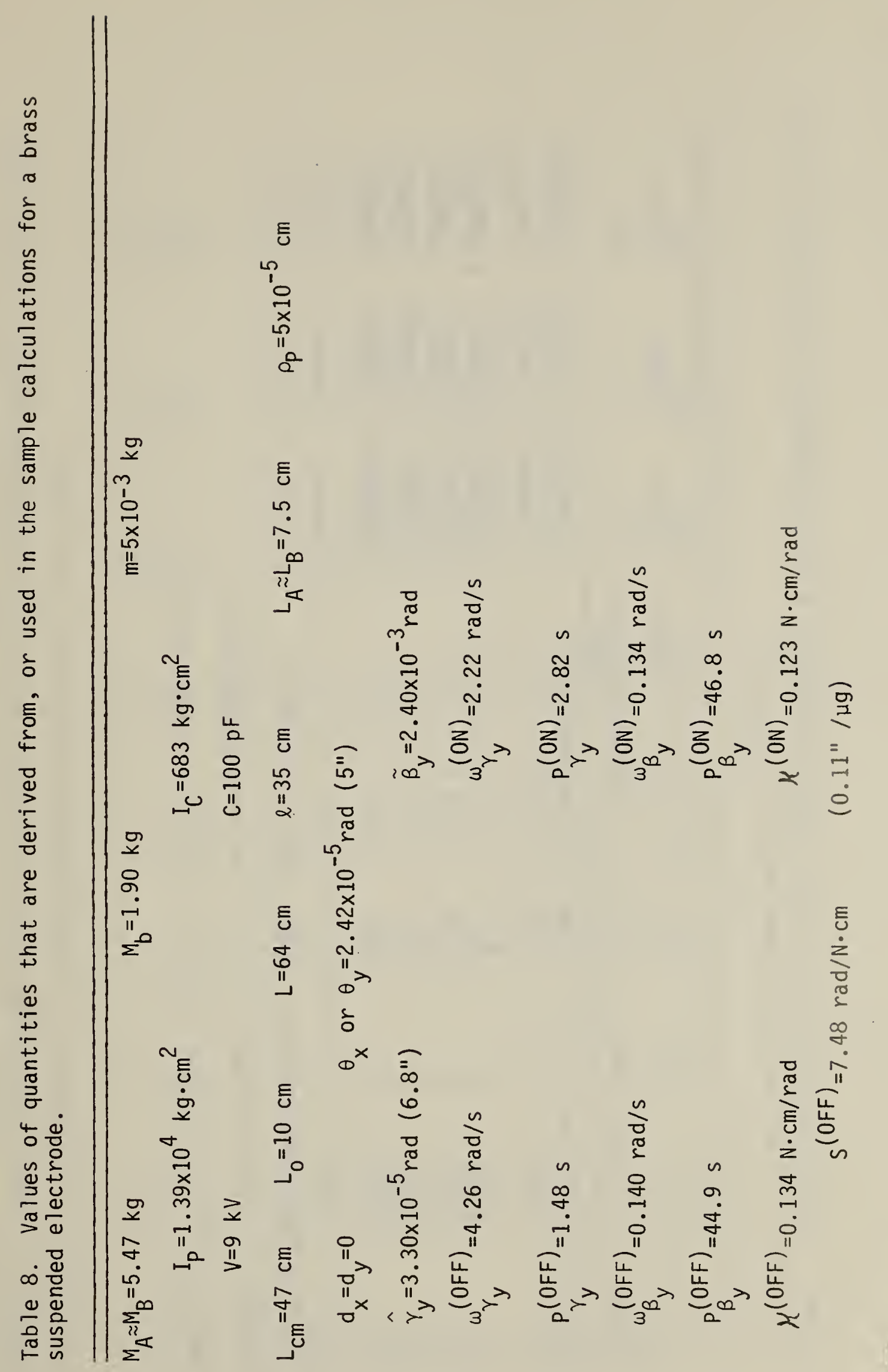




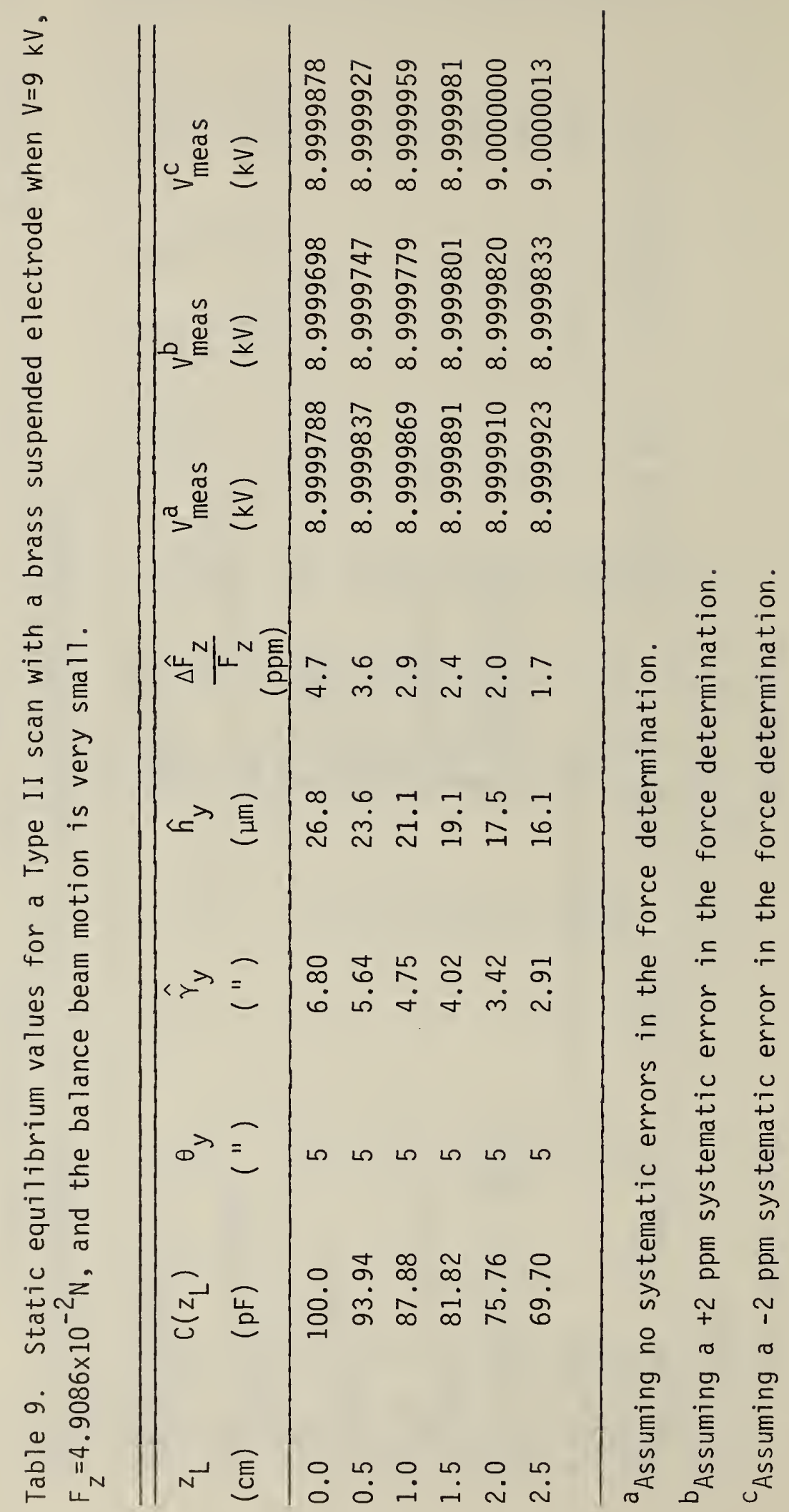




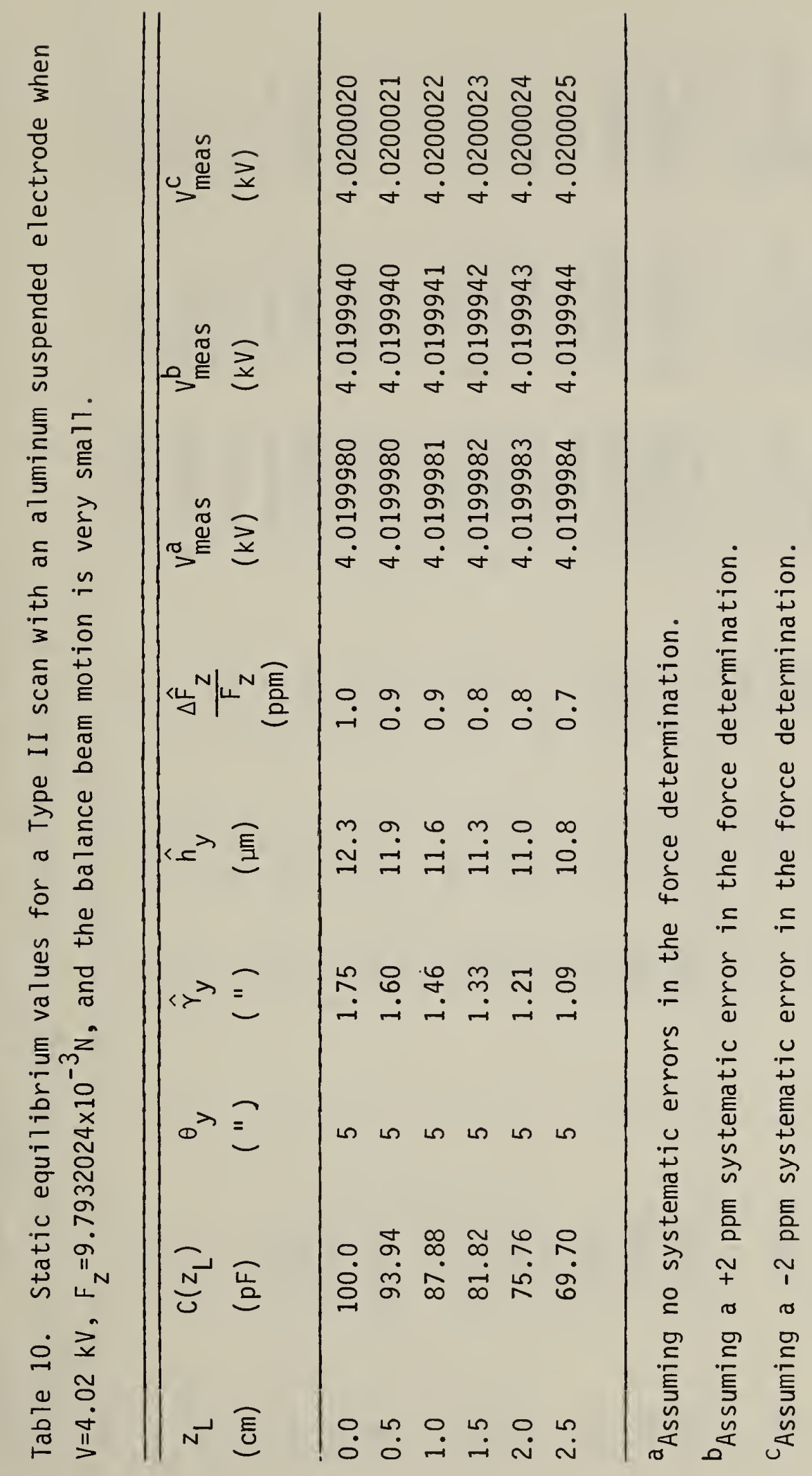




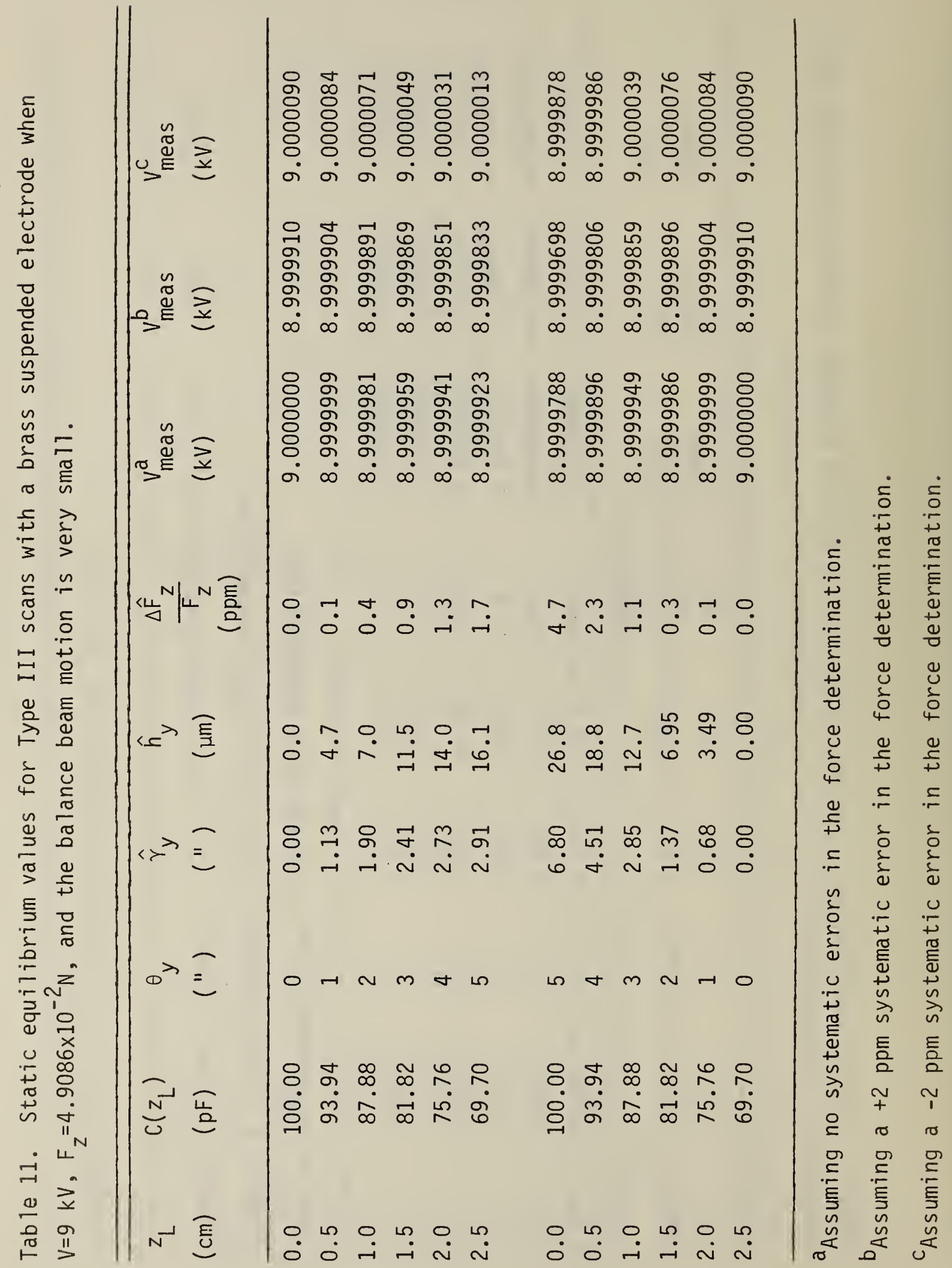




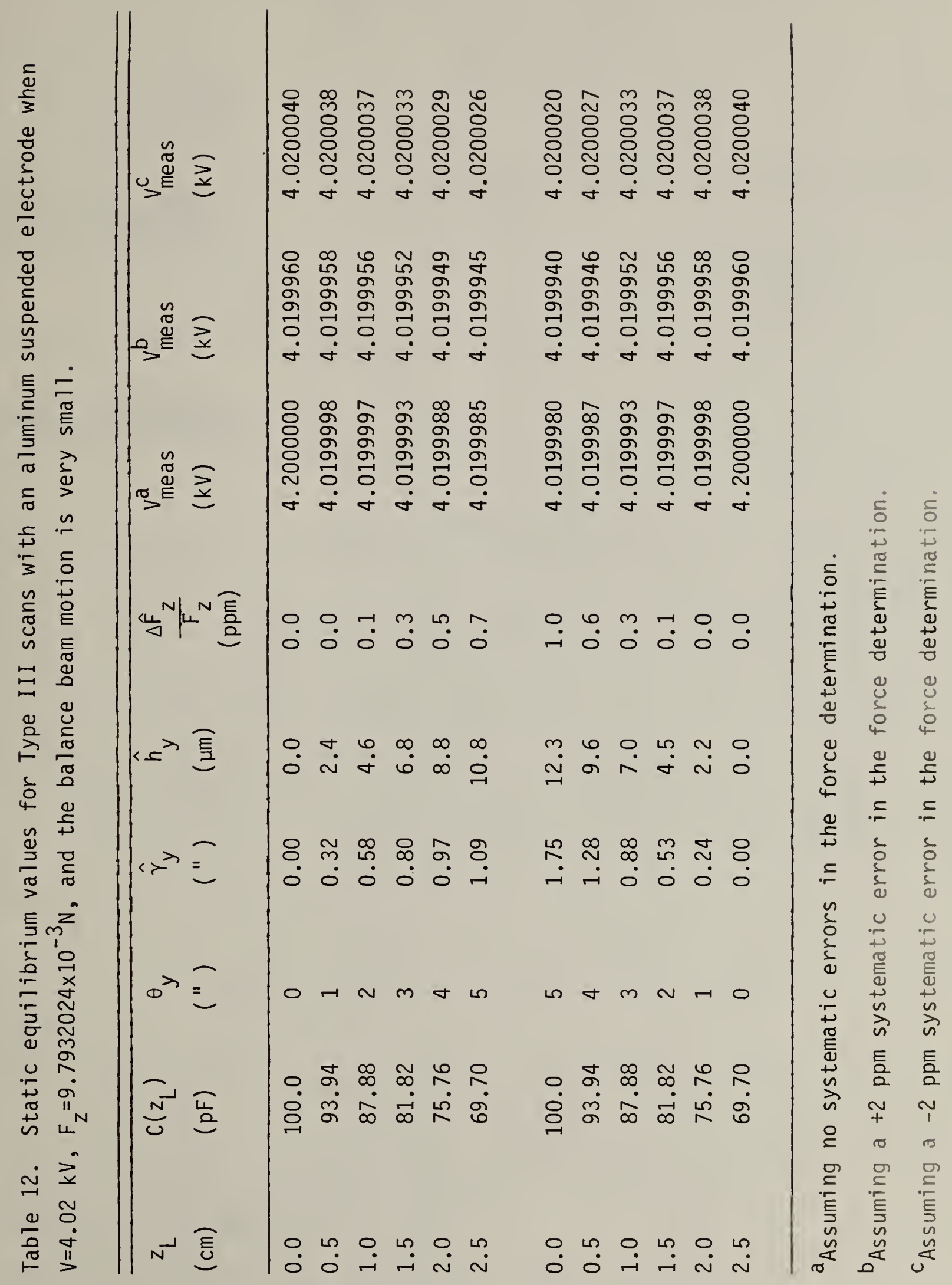




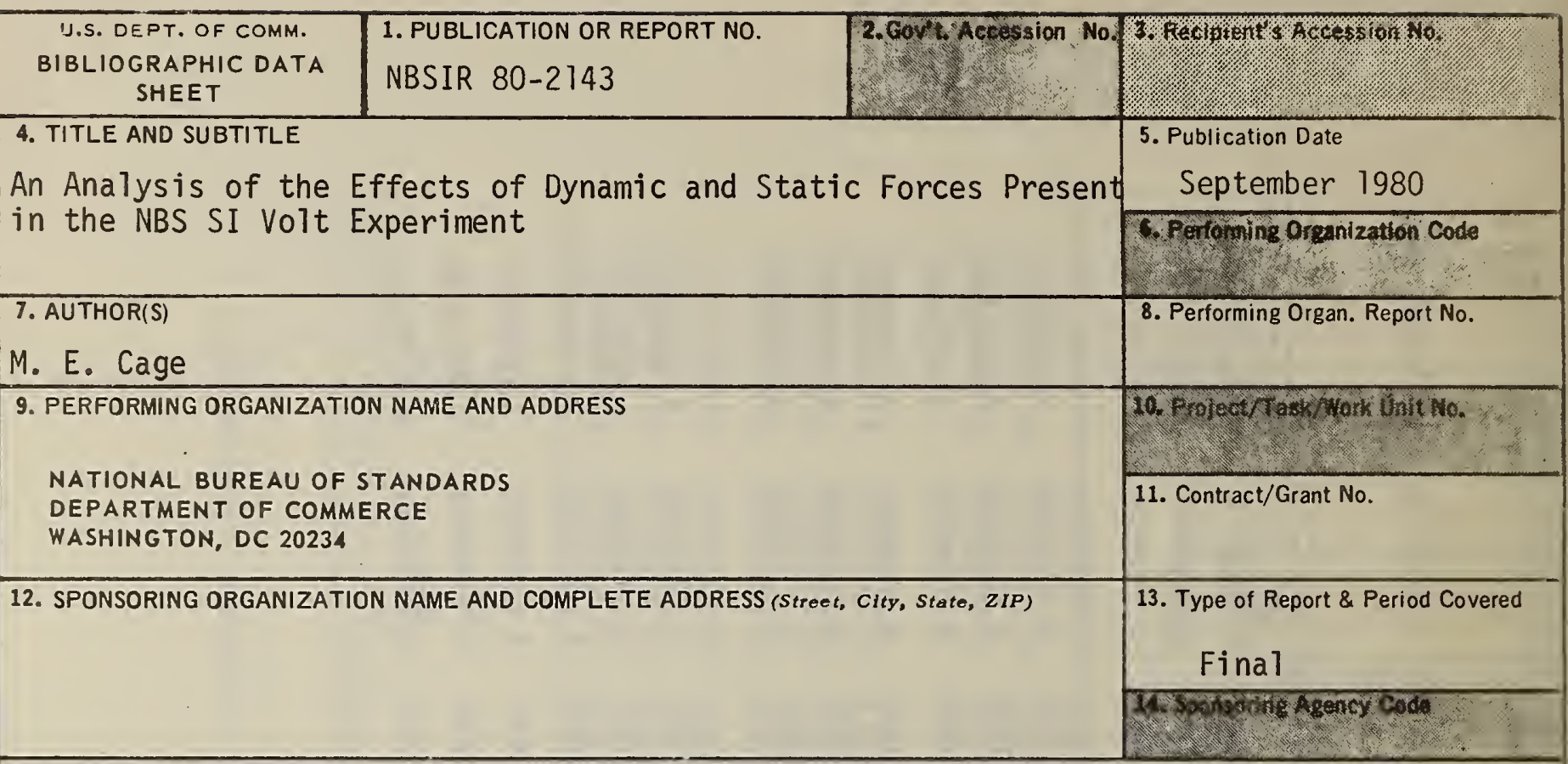

15. SUPPLEMENTARY NOTES

Document describes a computer program; SF-185, FIPS Software Summary, is attached.

16. ABSTRACT (A 200-word or less factual summary of most sigrificant informetion. If document includes a significant bibliography or literature survey, mention it here.)

An analysis of the NBS SI volt experiment has been made first to obtain algebraic expressions for the electrical forces present on the suspended electrode of the electrometer. These forces, and the gravitational forces, are used in the Principle of Virtual Work and D'Alembert's Principle to obtain the second order, non-linear, inhomogeneous, coupled differential equations of motion for the balance. Exact, analytical solutions of these equations of motion are obtained using small angle approximations and perturbation methods. Estimates are then made of the uncertainties that might result in both the slope and path integral methods in order to determine what requirements must be satisfied to reduce the systematic and random errors of the force determination and the capacitance measurements to acceptable levels so that the SI volt can be determined to within a few ppm.

17. KEY WORDS (oix to twelve entries; alphabetical order; capitallze only the first letter of the first key word unless a proper name; separated by semicolons)

Absolute volt; balance equations of motion; D'Alembert's principle; metrology; precision balance; precision electrical measurement

18. AVAILABILITY X Unlimited

For Official Distribution. Do iNot Release to NTIS

Order From Sup. of Doc. U.S. Government Printing Office, Washington, DC 20402, SD Stock No. SNỎ03-003-

X Order From National Technical Information Service (NTIS), Springfield, VA. 22161

\begin{tabular}{|l|c|}
\hline $\begin{array}{l}\text { 19. SECURITY CLASS } \\
\text { (THIS REPORT) }\end{array}$ & $\begin{array}{c}\text { 21. NO. OF } \\
\text { PRINTED PAGES } \\
\text { UNCLASSIFIED }\end{array}$ \\
\hline $\begin{array}{l}\text { 20. SECURITY CLASS } \\
\text { (THIS PAGE) }\end{array}$ & 218 \\
& $\$ 13.00$ \\
UNCLASSIFIED & USICE \\
\hline
\end{tabular}


